LA- $-11088-T$

DE88 000435
LA-1108B-T

Thesis

UC-34

Issued: October 1987

\title{
Statistical Theory of
}

Turbulent Incompressible Multimaterial Flow

Bryan Kashiwa 
University of Washington

Abstract

Statistical Theory of

Turbulent Incompressible Multimaterial Flow

by Bryan Andrew Kashiwa

Chairperson of the Supervisory Committee:

Professor David T. Pratt, Department of Mechanical Engineering

Interpenetrating motion of incompressible materials is considered. "Turbulence" is defined as any deviation from the mean motion. Accordingly a nominally stationary fluid will exhibit turbulent fluctuations due to a single, slowly moving sphere.

Mean conservation equations for interpenetrating materials in arbitrary proportions are derived using an ensemble averaging procedure, beginning with the exact equations of motion. The result is a set of conservation equations for the mean mass, momentum and fluctuational kinetic energy of each material. The equation system is at first unclosed due to integral terms involving unknown onepoint and two-point probability distribution functions. In the mean momentum equation, the unclosed terms are clearly identified as representing two physical processes. One is transport of momentum by multimaterial Reynolds stresses, and the other is momentum exchange due to pressure fluctuations and viscous stress at material interfaces.

Closure is approached by combining careful examination of multipoint statistical correlations with the traditional physical technique of $k-\epsilon$ modeling for single-material turbulence (Launder and Spalding 1974). This involves representing the multimaterial Reynolds stress for each material as a turbulent viscosity times the rate of strain based on the mean velocity of that material. The multimaterial turbulent viscosity is related to the fluctuational kinetic energy $k$, and the rate of fluctuational energy dissipation $\epsilon$, for each material. Hence a set of $k$ and $\epsilon$ equations must be solved, together with mean mass and momentum conservation equations, for each material. Both $k$ and the turbulent viscosities enter into the momentum exchange force.

Like the classical $k-\epsilon$ approach, formulation of the multimaterial $\epsilon$ equation requires postulates. In this theory some new postulates are posed. Examination of the equations in three engineering applications suggests the validity of the postulates made in the closure process. The applications are: a) calculation of the drag force on a sphere fixed in a uniform flow, b) calculation of the sestling rate in a suspension and c) calculation of velocity profiles in the pneumatic transport of solid particles in a pipe. 


\section{Table of Contents}

LIST OF FIGURES vii

LIST OF TABLES viii

CHAPTER I DEFINITIONS, BACKGROUND, OVERVIEW 1

1 Introduction, 1

2 Physical Features of Multimaterial Flows, 3

3 Theoretical Approaches to Multimaterial Flow, 8

4 Physical Approach to Single-Fluid Turbulence, 14

5 Overview of the Multimaterial Statistical Theory, 23

CHAPTER II STATISTICAL BASICS

1 Distribution Functions in the Multimaterial Theory, 27

2 Equation for the Pressure, 30

3 Ensemble Average Equations, 33

4 Summary, 40

CHAPTER III HOMOGENEOUS FLOW

1 One-Point Terms, 47

2 Two-Point Momentum Terms, 52

3 Two-Point Kinetic Energy Terms, 67

4 Interface Terms, 71

5 Summary, 73

CHAPTER IV NONHOMOGENEOUS FLOW

1 Multimaterial $k-\epsilon$ Theory, 76

2 Connection to Lundgren's Theory, 80

3 Nonhomogeneous Momentum Exchange, 83 
1 Introduction, 89

2 Basic Predictor-Corrector Method, 91

$3 \quad$ Accuracy and Stability, 94

4 Finite-Volume Ceneralization, 98

5 Multimaterial Implementation, 105

6 FORTRAN Computer Code, 112

CHAPTER VI APPLICATIONS

1 Drag Force on a Single Sphere, 120

2 Sedimentation of a Suspension, 124

3 Pneumatic Transport in a Pipe, 129

CHAPTER VII CONCLUSIONS

1 Summary of the Results, 139

2 Conclusions, 141

3 Recommendations for Extended Work, 142

A Numerical Calculation of Gradients, 148

B Relaxation Procedure, 150

C Listing of the FORTRAN Computer Code, 151

D Program for Solution of the Sedimentation Problem, 210 


\section{List of Figures}

Figure 1. Sketch of a particular realization of the distribution function $f$.

Figure 2. Sketch of the correlation coefficient $E\left(r_{12}\right)$

Figure 3. A spherical coordinate system.

Figure 4. Constant magnitude plot for the integrand of Eq. (3.20).

Figure 5. Constant magnitude plot for the integrand of Eq. (3.20) with and anisotropic function $g$.

Figure 6. Constant magnitude plot for the integrand of $\mathrm{Eq}$. (3.20) with an asymmetric furiction $g$.

Figure 7. Typical computational cell in two-dimensional logical space. 101

Figure 8. Flow formation in Couette motion.

Figure 9. Hydrodynamic entry length problem. 116

Figure 10. Hydrodynamic entry length problem. Error versus mesh spacing. 116

Figure 11. Flow in a channel at $\operatorname{Re}=12,300 . \quad 117$

Figure 12. Initial conditions for the Rayleigh-Taylor instability problem. $\quad 117$

$\begin{array}{ll}\text { Figure 13. Rayleigh-Taylor instability calculation. } & 118\end{array}$

Figure 14. Drag coefficient for a single fixed sphere in a uniform flow. Comparison of the multimaterial $k-\epsilon$ theory with experimental data.

Figure 15. Sedimentation speed versus liquid volume fraction. Comparison of the multimaterial $k-\epsilon$ cheory with experimental data.

Figure 16. Velocity profile data from the pneumatic transport experiments of Lee and Durst (1982).

Figure 17. Comparison of velocity profiles from the multimaterial $k-\epsilon$ theory with the pneumatic transport data of Lee and Durst (1982).

134

Figure 18. Comparison of no-slip (a) and free-slip (b) boundary conditions on the particle field.

Figure 19. Comparison of a calculation including the interpenetrational creation term (a) to the same computation omitting the creation due to interpenetration (c).

Figure 20. Distribution in root mean square velocity fluctuation for calculations $a$ and $b$, compared to the data of Lee and Durst (1982). 


\section{List of Tables}

Table 1. Conditions for six of the sedimentation experiments of Richardson and Zaki (1954).

128

Table 2. Comparison of the single-sphere free-fall velocity with the data of Lee and Durst (1982).

Table 3. Pressure gradients for pneumatic transport calculations. 


\section{Acknowledgments}

I would like to express my sincere thanks to Frank Harlow for standing by throughout, the work to provide the critical guidance needed to keep the formulation "physically correct", and from whom I have learned so much. To all of the members of Los Alamos National Laboratory group T-3, who each gave generous support during the work, I shall always be most grateful.

My thanks also go to Jerry Brackbill and especially Professors David Pratt, Ashley Emery and Clayton Crowe for reading and commenting on the work, as well as the remaining members of my University of Washington supervisory committee who, early on in the program, had faith in my ability to "do research".

B. A. Kashiwa

Los Alamos, New Mexico

Spring 1987 


\section{Chapter I}

\section{Definitions, Background, Overview}

\section{INTRODUCTION}

Multimaterial flows are a common occurrence in science, industry and everyday life. Examples inciucie the pulsating flow of blood in the human body, wheat grain transported by the turbulent flow of air in a pipe and sediments moved along the bed of a flowing stream. These examples each possess a common feature: dispersed entities of one material move with a velocity different from the surrounding fluid medium. The term "interpenetrating motion" describes this feature of multimaterial flows particularly well. The interpenetrating motion that is characteristic of flows containing mixtures of different materials, or different phases of the same material, gives rise to a class of physical phenomena unique to such flows. The depressed sound speed in a suspension of ges bubbles in a liquid is a well-known example of one such phenomenon. The combination of industrial and scientific importance with the contrasting behavior to single-material flows has made multimaterial flow theory a popular research topic.

For the purposes of this study a multimaterial flow is defined as any material movement exhibixing interpenetrating motion on a macroscopic scale. Notice that this definition classifies the motion of a liquid in a solid porous matrix as a multimaterial flow. However, this definition precludes, for example, the motion of a dye in water. In that case the particles of dye are so small that they move essentially with the velocity of the water, precluding any interpenetrating motion on a macroscopic scale. The foregoing definition classifies the motion of two immiscible liquids as a multimaterial flow, even though only a single phase of two materials is present. In the literature the terms "multiphase" (e.g., Harlow and Amsden 1975) and "two-phase" (e.g., Ishii 1975) have been used to describe multimaterial flows, even though only a single phase may be present in the problem. Because of this, the terms multiphase and multimaterial are used interchangeably here.

Interpenetration on a macroscopic scale must be distinguished from that which can occur on a microscopic or molecular scale. Interpenetration on a molecular scale occurs for example in the very thin regions of the earth's upper atmosphere when a rocket passes through the ionosphere. In this case the rocket exhaust eventually expands to the point where a streaming interpenetration occurs between ambient ions and molecules of combustion products. In this circumstance, a distinct interface between regions of pure material does not exist; the interaction between materials resides in the molecular mixing and subsequent collisions between individual molecules of different species. Molecular interpenetration is not the subject of this thesis. Here attention is limited to interpenetrating flow systems in which an interface is present that separates regions of pure materials. 
Despite the diverse industrial application and research attention that multimaterial flow problems have received in recent years, a fundamental theory, free of empiricism, is still lacking. For instance, a general method based on fundamental principles for determination of pipe pressure drop in the pneumatic transport of solid fragments is still unavailable. This means that engineers must rely heavily on experimental data for the design of such transport systems. For new applications the appropriate data may not be available.

The goal of this study is to lay the foundation for the development of a first-principles theory of multimaterial flow. In order to focus primarily on the fundamentals of momentum transport, consideration is limited to the case of incompressible materials with no changes in phase. This means that the effects of evaporation, condensation, chemical reaction, freezing and melting are all omitted from this study. The high-speed effects of shocks and rarefactions are also omitted. These restrictions are made only as a means of isolating the essential effects of momentum transport in multimaterial flows. Many industrially-important flow systems exist for which the limited theory is of considerable value. The transport of coal fragments in a hydraulic slurry is one example.

Many industrial multimaterial flows are composed of a turbulent fluid carrying suspended fragments of solid in a pipe or duct. The definition of turbulence in a fluid requires familiarity with the concepts of mean (average) flow properties and instantaneous (local) flow properties. The definition of a mean flow preperty depends upon what is important to the observer. For example, the average flow speed and average pressure drop in a length of pipe are generally of interest to the cngineer responsible for pumping a single fluid in a pipe. The engineer is well cware that at any instant or at any location in the pipe the pressure gradient and velocity may differ considerably from the average values that are really of interest. The departure of local properties from the mean properties, called fluctuations, are of concern only if they are sufficient in magnitude to affect the mean flow. This idea provides a useful definition of turbulence in the flow of a single fluid. In this study, a flow wili be considered turbulent when fluctuations in the velocity and pressure are large enough to produce a significant effect on the mean flow quantities of interest.

Muitimaterial flow is defined here as flow exhibiting interpenetrating motion on a macroscopic scale. This interpenetrating motion is inherently accompanied by fluctuations in the velocity and pressure that have an important effect on the mean flow behavior, defined by almost any obs rver. For example consider the motion of a single metal sphere falling under the force of gravity in a viscous fluid. An observer may be interested in the rate at which the sphere descends in the nominally motionless fluid. In this case, the motion of the sphere is determined completely by the fluctuations in the pressure and velocity of the fluid.

In this thesis, the foregoing definition of fluid turbulence for the single fluid case is considered relevant to the multimaterial case. With this definition, one can 
readily conclude that multimaterial flows are usually turbulent. That is to say, an interpenetrating flow has a local pressure and a local velocity field both of which fluctuate frorn the mean in such a way that the average motion is influenced. In this sense even a viscous flow creeping past a sphere is a turbulent flow.

The next section establishes some very essential physical features of multimaterial flows. These features are deduced partly on physical grounds and partly on the basis of analytical results from the literature. The bulk of these results pertain to the force on a single rigid body in a well-defined flow field. Section 3 is a review of the main theoretical nuethods that have been used in the various developments in multimaterial flow theory. Section 4 provides a brief description of the ways by which the theory of single-fluid turbulence has been approached in the literature. In Sec. 4, the physical approach is highlighted as opposed to some of the more mathematical approaches that have been used in turbulence theory. Finally, Sec. 5 gives an overview of the set of methods by which the theory is developed in the present work. There it is seen that the methods employed here are a mixture of techniques from statistical mechanics and physically based modeling methods that have been successful in the theory of single-fluid turbulence transport.

\section{PHYSICAL FEATURES OF MULTIMATERIAL FLOWS}

This section contains a review of relevant analytical and experimental results from the literature to provide the essential background knowledge for later developments. The literature contains a vast collection of information pertaining to the analysis of fluid flow in the presence of a single submerged body. A great deal is known, for example, about the nature of fluid forces exerted on a rigid sphere in an incompressible fluid at low relative Reynolds number (Batchelor 1967). This knowledge is gained by way of careful analytic study of the Navier-Stokes equations for a well-defined fluid flow field and well-defined boundary conditions on the submerged body.

The statistical ensemble provides an important means by which the knowledge of single-body dynamics can be connected to the many-body dynamics that can occur in a general multimaterial flow. A statistical ensemble is a large conceptual set of flow systems that are identical in all average respects of initial and boundary conditions. The systems differ only in the precise way that certain quantities deviate from the average. In general, the deviations from the average are sufficient to cause each member of the ensemble to differ from one another. The statistical ensemble is used in defining the ensemble average, a precise definition of which is given in the next chapter. For now, the important point is that the system consisting of a single rigid sphere in an infinite incompressible fluid is a valid member of a statistical ensemble. Furthermore, the initial and boundary conditions for each of the members of the ensemble can be identical in all respects, including the fluctuating details. In this case the behavior of any member of the ensemble is identical to every other member and is therefore identically equal to the average 
behavior.

Thus any theory that is capable of describing the dynamics of a general multimaterial flow must contain, as a special case, the dynamics of a single particle in a fluid. This fortunate state of affairs enables deduction of three important features of multimaterial flows. These are:

1. In all multimaterial flows, a force is exerted on one material as a result of average interpenetrating motion relative to another material.

2. Multimaterial flows are always rotational in the presence of a nonzero average interpenetrating motion.

3. Multimaterial flows are always statistically anisotrcpic, and may be statistically asymmetric in the presence of an average interpenetrating motion.

The remainder of this section is a discussion of how these essential features of multimaterial flows can be deduced. The main supporting evidence for the conclusions is supplied by results from analytic calculations of the force on a regular rigid body in a well-defined flow field.

To begin, the first physical feature of multimaterial flows will be established. There are four known ways that a net force on one material can arise from a net interpenetrating motion. These are large-scale fluid rotation, viscous stress at a material interface, small-scale fluid rotation and inertia of the displared fluid.

A standard result from hydrodynamic theory is the absence of a net force on a rigid body of any shape submerged in a uniform flow of an ideal (nonviscous, incompressible) fluid. Only if there is a net rotation in the fluid can an ideal fluid exert a net force on a submerged body (Currie 1974, 164). One can easily visualize, knowing the principle of Bernoulli, that a rotating ideal fluid flowing past a rigid body will necessarily have a pressure deficit on one side of the body. This is a simple consequence of the fluid flowing faster on one side of the body, which creates a lift force.

This can be quantified by considering, for example, a circular cylinder moving horizontally to the right in an infinite ideal fluid, in two space dimensions. If the fluid at infinity is stationary, both the horizontal force (drag) and the vertical force (lift) are zero. However if the fluid with density $\rho$ is rotating such that a circulation of strength $\Gamma$ is superimposed on the rectilinear flow $-U$, the drag force is still zero but there is a lift force per unit length of

$$
-\rho U \Gamma
$$

on the cylinder. This is the so-called Kutta-Joukowski law (Currie 1974, 94) and is an exact result from potential flow theory. Here the circulation is defined

$$
\Gamma=\int(\nabla \times \mathbf{u}) \cdot \hat{\mathbf{n}} d A
$$


where the integral is over the area of the cylinder with outward normal $\hat{\mathbf{n}}$, and $\mathbf{u}(\mathbf{x})$ is the local fluid velocity. The drag force is zero because the flow is symmetric about the vertical axis drawn through the center of the cylinder. The lift force is nonzero because the flow is asymmetric about the horizontal axis through the center of the cylinder. In Chapter III this asymmetry is shown to be of crucial importance to the calculation of forces between materials in general multimaterial flow situations.

In a real fluid the viscous stress exerts a force on a submerged body of any shape, even in a uniform flow, providing there is a nonzero relative mean velocity (interpenetration). For a uniform steady relative flow speed $U$, the magnitude of the drag force on a sphere of radius $s$, called Stokes drag, is (Batchelor 1967)

$$
6 \pi \mu s U \text {, }
$$

where $\mu$ is the viscosity of the incompressible fluid, and the direction of the force is parallel to the relative motion. The Stokes drag force neglects inertial effects and is only accurate when viscous forces in the fluid dominate. The measure of this dominance is, of course, the relative Reynolds number $R e_{v}$ defined

$$
R e_{v}=\frac{2 s U}{\nu},
$$

where $\nu$ is the kinematic viscosity of the fluid. When $R e_{v}$ becomes comparable to unity, flow separation may occur. With separation, there is a pressure deficit on the downstream side of the body, causing a net drag force on the body. The flow energy associated with the pressure deficit is deposited into fluid structures (called eddies) that are usually small compared with the body itself. Thus the force due to flow separation and small-scale fluid rotation are connected phenomena.

When flow separation occurs, one must normally resort to empirical means to determine the force on a submerged body. The total force is usually computed using the experimentally determined drag and lift coefficients $C_{d}$ and $C_{l}$ respectively. The drag coefficient is usually defined such that the drag component of the force $F_{d}$ is

$$
F_{d}=\frac{1}{2} \rho U^{2} A C_{d}
$$

and similarly the lift coefficient is defined such that the lift component of the force $F_{l}$ is

$$
F_{l}=\frac{1}{2} \rho U^{2} A C_{l}
$$

where $A$ is the area of the body facing the relative flow (Currie 1974). The sense of $F_{d}$ is parallel to the relative motion and the sense of $F_{l}$ is perpendicular to the relative motion.

If the motion of a submerged body relative to a nonviscous fluid is timevarying, the inertia of the displaced fluid causes a force opposing acceleration of 
the body. The momentum equation for the body contains a term that quantifies this force. This term is called the added mass term in the literature (Maxey and Riley 1983). In a viscous fluid, another time-unsteady part of the force arises as the boundary layer near the material interface develops toward its steady configuration. The momentum equation term that describes this effect has been calied the Basset history term (Maxey and Riley 1983). Both the added mass and Basset history terms can cause an increase in the force on a submerged body above that which would be obtained if the relative rate of interpenetration is assumed steady. Hence the fourth contribution to the exchange force is the time-unsteady behavior in fluid displacement and boundary layer development.

The fact that there is always a force on a submerged body in motion relative to a real fluid, and the fact that a single body in a sea of fluid is a valid member of a statistical ensemble, enable deduction of the first main property of the multimaterial flows. Whenever an average interpenetration takes place, the components of a multimaterial mixture will experience an average force opposing the interpenetration (drag). In addition there may be a component of force perpendicular to the interpenetration (lift). Because total momentum is conserved, the average force on material one (due to material two) will be equal and opposite to the average force on material two (due to material one). The force is therefore properly called the exchange force. An exchange force is present regardless of the physical nature of the materials, providing a material interface exists in the flow and there is a nonzero relative average velocity. Hence the rule holds for the case of many rigid bodies suspended in a fluid as well as the case of many deformable bodies (bubbles) in a fluid.

The exchange force can arise as a result of four fundamentally different effects. These are fluid rotation on a scale large compared to the suspended entities (circulation), viscous stress at material interfaces, fluid rotation on a scale small compared to suspended entitits (separation) and time-unsteady relative flow. The effect of large-scale fluid rotation can be thought of as contributing a long-range part of the total exchange force. This is because the circulation in the fluid depends on the correlation in velocity over distances that are large compared to the size of a suspended body. The forces caused by viscous stress at interfaces, separation and time-unsteady effects all occur on a size scale comparable to the size of a suspended body. For this reason, these parts of the force can be considered to be short-range forces.

Another ir:portant short-range exchange force is present when the dispersed entities are composed of bubbles of an immiscible fluid. In this case, the surface tension force at the material interface can play an important role. This is the role of establishing the size scale of the dispersed entities themcelves; an important role because the other short-range forces depend on this size scale. An experimental result is frequently used to estimate the size scale in a fluid-fluid multimaterial flow. This result is reported in the form of the ratio of the kinetic energy in the 
flow to the potential energy contained in surface tension. This ratio is called the Weber number $W e$, defined

$$
W e=\frac{\rho U^{2}}{\sigma / s},
$$

where $\rho$ is the density of the continuous fluid and $\sigma$ is the surface tension (energy per unit area) between the fluids. For many fluid-fluid pairs, and for a wide range of relative Reynolds numbers, $W e=10$ provides a good estimate of the steady-flow entity size $s$ (Hetsroni 1982, Ch. 4).

The second main feature of interpenetrating flow arises partly as a consequence of the first. It is well known that vortic $y$ in a real fluid is generated at boundaries because of the no-slip condition on the velocity at a surface (Currie 1974). The present work has been confined to interpenetrating flows in which a material interface is a prominent characteristic. Thus any interpenetration will produce fiuid shear layers near the material interfaces. If one material is a collection of suspended rigid bodies, the vorticity generated at the surface of the bodies may be a significant feature of the local fluid velocity field. If the vorticity generated is sufficiently large, flow separation occurs, giving rise to part of the force discussed in the previous paragraphs.

The small-scale rotation that is engendered at material interfaces can be : perimposed upon a large-scale rotation. This large-scale rotation can occur in different ways. Consider for example a flow that is composed of a continuous fluid with suspended rigid bodies. If the rigid bodies are closely spaced in one region and sparsely spaced in an adjacent region, the fluid will tend to pass through the sparse region more easily than it passes through the crowded region. This establishes a mean rotation in the continuous fluid that is measured on a scale large compared to the size of the suspended bodies. Another source of large-scale rotation is the intense shear that can occur in the boundary layer, near the wall of a pipe for example.

Thus the presence of material interfaces in an interpenetrating flow assures the presence of rotation on a scale commensurate with the scale of the interfaces themselves. Furthermore, this rotation can be superimposed on a large-scale rotation caused by variable entity spacing or boundaries of the flow domain. Therefore on these physical grounds, one can conclude that multimaterial flows are always rotational when a net interpenetration takes place.

The final main feature of multimaterial flows is also understood on physical grounds. Consider the case of a single rigid sphere falling steadily in a viscous fluid under the force of gravity. An observer riding with the sphere will see the same fluid velocity, and its change with distance from the sphere, by looking along a ray perpendicular to the direction of motion of the sphere. However the observer will see a very different velocity and its variation with distance if looking ahead or behind the sphere. This will always be the case whether the flow is time-steady or not, regardless of the nature of the rigid body or the properties of the fluid. 
Thus the observer collecting velocity data for calculating ensemble averages will always find that the statistics generated are anisotropic, even if the flow systern is homogeneous, provided there is an average interpenetration. The only way isotropy can be approached is if there is no average relative motion, or if there is no interpenetration at all. Earlier in this section, large-scale rotation was seen to be responsible for a force caused by an asymmetry in the velocity field in an ideal fluid passing around a cylinder. A similar asymmetry will be reflected in the ensemble average velocity statistics collected by the observer riding the falling sphere. In the latter case the asymmetry leads to a drag force rather than a lift force as it did in the case of the cylinder with circulation. Hence one can conclude that besides the fundamental anisotropy, multimaterial flows can be statistically asymmetric as a result of fluid rotation on either a large or small scale compared to the size of dispersed entities.

The importance of the nonzero exchange force, rotationality and anisotropy of multimaterial fiows is the following. Knowledge of the presence of the exchange force, and in particular a knowledge of its fundamental sources, provides an essential guide by which to develop a set of equations designed to represent the dynamics of multimaterial flows. Because rotationality on various size scales can compose a part of the exchange force, knowledge of its origin is important in making the approximations needed to complete the theory. The feature of anisotropy is also essential to the development because it establishes a direction for the action of the exchange force. The foregoing discussion suggests that the direction of the exchange force is primarily along the direction of the interpenetrating motion itself.

\section{THEORETICAL APPROACHES TO MULTIMATERIAL FLOW}

The goal of multimaterial flow theory is to provide a relationship between the average dynamical behavior of flow systems and the instantaneous local events that affect the average flow. This is the same goal that is attained in the elementary kinetic theory of gases, where the average behavior of the gas is predicted by consideration of the motion and interaction of individual molecules that are idealized as rigid spheres. The purpose of this section is to provide a brief review of the way in which prior workers have approached the goal of multimaterial flow theory. The difficulty with doing this is that interpenetrating flows of the type considered in this thesis arise in a great number of engineering and scientific areas. This makes the literature not only extensive but also diverse in terms of the application to specific problems.

In order to systematize the review in this section, previous approaches to multimaterial flow theory are classified here according to the mathematical representation from which the average behavior is to be derived. This enables a distinction to be drawn between the present approach and the prior work. There are four classes of mathematical representation that will be discussed here. The approaches asso- 
ciated with each representation will be referred to as the Multifluid-Continuum, Boltzmann-Continuum, Fokker-Planck-Continuum and Multimaterial-Boltzmann approaches.

The first class of mathematical representation is by far the most widely used for multimaterial flow tineory. This is the Multifluid-Continuum approach. (Murray 1965, Anderson and Jackson 1967, Drew 1971, Whitaker 1973, Harlow and Amsden 1975, McTigue 1982, Needham and Merkin 1982.) This representation considers the multiple materials as interpenctrating continua. A significant initial assumption that is usually made in regard to size scales is that the relevant scale of dispersed entities in the flow $s$ is small compared to a control volume of measure $L_{c}$, which in turn is small compared to the macroscopic measure of the flow system $d$. This constraint may be stated by

$$
s \ll L_{c} \ll d
$$

The conditions of $(1.8)$ must be satisfied if statistically significant averages are to be obtained from the instantaneous conservation equations written for the control volume (Whitaker 1973).

Consider the case of two-material flow without chemical reaction, evaporation, or condensation. Let the effects of mean fluid deformation (viscosity) be negligible compared to the exchange force. In this case, the equations for circumstances satisfying (1.8), are the mass conservation equation

$$
\rho^{(k)}\left[\frac{\partial \theta^{(k)}}{\partial t}+\frac{\partial}{\partial x_{j}} u_{j}^{(k)} \theta^{(k)}\right]=0,
$$

and the momentum equation

$$
\begin{aligned}
\rho^{(k)}\left[\frac{\partial}{\partial t}\left(\theta^{(k)} u_{i}\right)+\frac{\partial}{\partial x_{j}} u_{j}^{(k)}\left(\theta^{(k)} u_{i}\right)\right]= & -\theta^{(k)} \frac{\partial p}{\partial x_{i}} \\
& +K\left(u_{i}^{(l)}-u_{i}^{(k)}\right),
\end{aligned}
$$

in which $k=1,2$ and $l=2,1$ (Harlow and Amsden 1975). In (1.9) and (1.10), $\theta^{(k)}(\mathbf{x}, t)$ is the fraction of the volume occupied by material $k$ at any instant $t$ and position $\mathbf{x}, \mathbf{u}^{(k)}$ is the volume averaged velocity of material $k$ at $(\mathbf{x}, t), \rho^{(k)}$ is the constant density of pure material $k, p(\mathbf{x}, t)$ is the pressure and $K$ is a positive function.

By definition

$$
\theta^{(1)}+\theta^{(2)}=1,
$$

so that using (1.9), and the fact that the term involving $K$ sums to zero by adding the momentum equations for the two materials, the unknown $p(x, t)$ can be eliminated from (1.10). This leaves $K$ the only undetermined quantity. The quantity 
$K$ is called the momentum exchange function. It is a positive coefficient that describes the rate at which the velocity of interpenetration $\left(u_{i}^{(l)}-u_{i}^{(k)}\right)$ diminishes with time as a result of the volume averaged force that exists between the two materials.

Within the context of the continuum representation, a constitutive relationship between $K$ and the independent variables must be postulated. In general, the miomentum exchange function is a tensor of (at least) order two (Bear 1972),

$$
K_{i j}=G\left(\theta^{(l)}, \theta^{(k)},\left|\mathbf{u}^{(l)}-\mathbf{u}^{(k)}\right|, \frac{\partial \theta^{(l)}}{\partial x_{i}}, \frac{\partial \theta^{(k)}}{\partial x_{i}}, \ldots\right)
$$

where $G$ represents some function of the independent variables and their derivatives. This relationship can only be developed on physical grounds using, for example, knowledge of the effects causing the exchange force, as reviewed in the previous section. Only for very restricted circumstances can a constitutive relation for $K_{i j}$ be written down with confidence. This is the first difficulty with the Multifluid-Continuum representation.

The second difficulty is that Eqs. (1.9) and (1.10), although already averaged over the control volume satisfying (1.8), are instantaneous equations and therefore must be averaged again to obtain the desired average behavior (Drew 1971). The most frequent approach to this is to assume that turbulent fluctuations are negligible so that Eqs. (1.9) through (1.12) give directly the average dynamical behavior. This assumption is potentially very seriously defective, particularly because multimaterial flow is usually turbulent, in the sense that fluctuations from the mean can have a significant influence on the average behavior. A much less frequently used approach is to perform a so-called Reynolds decomposition and averaging of Eqs. (1.9) and (1.10) (Elghobashi and Abou-Arab 1983, Besnard and Harlow 1985). This introduces the quintessential closure problem familiar from the theory of single-fluid turbulence; the averaging produces additional unknowns in the form of averages of products in the fluctuations, which are in general nonzero.

The second mathematical representation that has been used to develop multimaterial flow equations is relevant to the case of small particles in a continuous fluid. In this approach, a Boltzmann equation is used for the probable number of small particles per unit volume of phase space. The fluid is considered to be a continuum acted upon by the average force due to the presence of the particle. For this reason, the method is referred to here as the Boltzmann-Continuum approach (Travis, Harlow and Amsden 1976).

To understand this better, consider the case of uniform spherical particles of mass $m$ in a nonviscous incompressible fluid. Now define the number distribution function $N(\mathbf{x}, \mathbf{v}, t)$ such that $N d v$ is the probable number of small particles with velocity between $v$ and $v+d v$ at $(x, t)$, so that (1.8) is satisfied. If body forces (such as gravity) and direct collisions between the particles are absent, the evolution 
equation for $N$ is the collisionless Boltzmann equation

$$
\frac{\partial N}{\partial t}+v_{j} \frac{\partial N}{\partial x_{j}}+\frac{1}{m} \frac{\partial}{\partial v_{j}} F_{j} N=0,
$$

in which $F$ is the force acting on an individual particle as a result of motion relative to the fluid (Thavis, Harlow, and Amsden 1976). The conservation equations for the average behavior of the particles are obtained by calculating moments of Eq. (1.13).

Since particles are conserved,

$$
\int_{-\infty}^{\infty} N d \mathbf{v}=n
$$

where $n$ is the average number of particles per unit volume in the system. The average volume fraction is

$$
\left\langle\theta^{(1)}\right\rangle \equiv \frac{4}{3} \pi s^{3} \int_{-\infty}^{\infty} N d \mathbf{v},
$$

where $s$ is the radius of the spherical particles, and the average momentum of the particles, per unit total volume, is

$$
\left\langle\theta^{(1)} u_{j}\right\rangle \equiv \frac{4}{3} \pi s^{3} \int_{-\infty}^{\infty} N v_{j} d v
$$

so that the average particle velocity $\left\langle\mathbf{u}^{(1)}\right\rangle$ can be defined

$$
\left\langle u_{j}^{(1)}\right\rangle \equiv \frac{\left\langle\theta^{(1)} u_{j}\right\rangle}{\left\langle\theta^{(1)}\right\rangle} .
$$

Using (1.15) and (1.17), the moment of (1.13) corresponding to $m=\frac{4}{3} \pi s^{3} \rho^{(1)}$ is the average mass conservation equation for the particles

$$
\rho^{(1)}\left[\frac{\partial\left\langle\theta^{(1)}\right\rangle}{\partial t}+\frac{\partial}{\partial x_{j}}\left\langle u_{j}^{(1)}\right\rangle\left\langle\theta^{(1)}\right\rangle\right]=0
$$

The average momentum equation for the particulate field is obtained by computing the $m v_{i}$ moment of (1.13). The result is

$$
\begin{aligned}
\rho^{(1)}\left[\frac{\partial}{\partial t}\left\langle\theta^{(1)} u_{i}\right\rangle+\frac{\partial}{\partial x_{j}}\left\langle u_{j}^{(1)}\right\rangle\left\langle\theta^{(1)} u_{i}\right\rangle\right]= & -\left\langle\theta^{(1)}\right\rangle \frac{\partial\langle p\rangle}{\partial x_{i}} \\
& +\iint N(p-\langle p\rangle) \hat{n}_{i} d a d \mathbf{v} \\
& -m \frac{\partial}{\partial x_{j}} \iint C_{i}^{(1)} C_{j}^{(1)} N d \mathbf{v},
\end{aligned}
$$


where $\mathbf{C}^{(1)}=\mathbf{v}-\left\langle\mathbf{u}^{(1)}\right\rangle,\langle\boldsymbol{p}\rangle$ is the average pressure, and $\hat{\mathbf{n}} d a$ is the outward normal element of surface for one of the particles. The second term on the right of (1.19) is the average momentum exchange term. This corresponds to the second term on the right of Eq. (1.10), averaged. The third term on the right of (1.19) is a consequence of the averaging, and resembles a Reynolds stress. Both the second and third term on the right of Eq. (1.19) must be modeled in some way in order to close the equations. This makes the Boltzmann-Continuum approach appear to be no better off than the Multifluid-Continuum approach. There have been however, a few attempts at obtaining numerical solutions to Eq. (1.13), or some approximation to (1.13), that have produced useful results. Notable among these is the study by Lourenco, et al. (1982) who solve Eq. (1.13) together with average continuum equations for the fluid under time-steady turbulent flow conditions.

The Boltzmann-Continuum approach has also been used in the study of liquid droplet sprays. For this problem, the phase space of $N$ is extended to include coordinates that allow the statistical description of properties such as size and temperature, in addition to velocity, at $(x, t)$ (Williams 1985). The corresponding Boltzmann-like equation, called the spray equation, has been solved numerically in varying degrees of approximation (Westbrook 1977, O'Rourke 1981).

There is also a subset of the Boltzmann-Continuum approach that is noteworthy. This subset is realized by assuming that, in a control volume satisfying (1.18), the distribution in particle velocities among a given "class" of particles is infinitely sharp about the mean. A class of particles is a group with identical characteristics such as size. In the foregoing example there is a single class of particles with size $s$ and mass $m$. The approximation is then

$$
N \approx N_{0} \delta\left(\mathbf{v}-\left\langle\mathbf{u}^{(1)}\right\rangle\right)
$$

where $N_{0}$ is the number of particles per unit total volume in the ciass, and $\delta$ is the Dirac delta function. With (1.20) the mv moment of (1.13) becomes

$$
m N_{0} \frac{d}{d t}\left\langle\mathbf{u}^{(1)}\right\rangle=\mathbf{T},
$$

in which

$$
\left\langle\mathbf{u}^{(1)}(t)\right\rangle=\frac{d}{d t} \mathbf{x}^{(1)}(t),
$$

where $\mathbf{u}^{(1)}(t)$ is the velocity along the center-of-mass motion of the particle class, whose position is $x(t)$. Equations (1.21) and (1.22) are the Lagrangian equations of motion for the class of particles. The force on the particles $F$, due to the continuous fluid, is a function of the mean particle velocity $\left\langle u^{(1)}\right\rangle$ and the local, instantaneous fluid velocity. In general, $N_{0}$ can be a function of time, provided an equation for its change with time can be derived. In most cases $N_{0}$ has been considered to be a constant. 
The negative of the fluid-particle force becomes a source term to the momentum equation for the fluid. This source term corresponds to the exchange term of Eq. (1.13) written for the fluid. The multimaterial flow equations are now given by (1.21) and (1.22) for the particles, and (1.12) and (1.13) for the fluid. Solution of these equations, together with appropriate relations for the fluid-particle force, has been called the trajectory approach (Crowe 1982). Evidently the first use of this particular subset of the Boltzmann-Continuum approach is due to Crowe and Pratt (1972). A variety of other workers have used similar methods since then; mostly for dilute particle-laden gas flows or thin droplet sprays (Dukowicz 1980, O'Rourke 1981).

The Boltzmann-Continuum approach is useful from a theoretical standpoint because it provides a step toward quantifying the momentum exchange function $K$ appearing in the Multifluid-Continuum formalism. However, this can still only be done with confidence for very thin (dilute) suspensions in which the particles can be considered to behave in an isolated manner.

Both the Multifluid-Continuum approach and the Boltzmanri-Continuum approach can be called deterministic because the momentum exchange force is precisely determined by some function of the average flow conditions. In contrast one can imagine that turbulent fluctuations could, at some length scale, introduce a random component to the exchange force. This way of thinking parallels the idea used in the theory of Brownian motion, in which a small particle in a fluid is subjected to a random force via bombardment by thermally fluctuating fluid molecules (Chandrasekar 1943). If the particle-fluid force in Eq. (1.21) is divided into a mean part and a random part, the equation said to be stochastic, and the corresponding particle evolution equation for $N$ becomes the Folkker-Planck equation (Chandrasekar 1943). Thus the stochastic method is referred to here as the Fokker-Planck-Continuum approach because the fluid is again considered to be a continuum co-occupying any control volume together with the small particles.

This approach is much less used in the literature, presumably because of its limited applicability (Buyevich 1971, Fan 1985). The method is limited because the random part of the particle-fluid force is usually considered isotropic. The velocity fluctuations in a turbulent fluid only approach isotropy at the very smallest size scales in the flow. Thus an isotropic component of the particle-fluid force is only relevant in cases where the particle size is small enough to be significantly affected by the smallest scale structure of the flow.

The mathematical representation used in the present work makes use of a Boltzmann-like equation for each material in a multinaterial flow. For this reason the present method is referred to here as the Multimaterial-Boltzmann approach. In this the probability distribution functions for velocity of each material are defined in a way that does not require a condition on scales such as (1.8). The average behavior of the materials is obtained by taking moments of the evolution equations for the distribution functions. The resulting average equations for mo- 
mentum conservation show that the momentum exchange term contains integrals over the two-point probability distribution functions.

There are two main advantages of the Multimaterial-Boltzmann approach over the other three approaches discussed in this section. The first is that a reasonable appreximation to the tensor momentum exchange ccefficient $K_{i j}$ can be obtained by modeling, in effect, the two-point probability distribution functions, on physical grounds. The second advantage is that the form of the average equations is very closely analogous to the ensemble averaged Navier-Stokes equations for a single incompressible turbulent fluid.

The first of these advantages is that a systematic way is provided for including the essential physical features of multimaterial flows into a generally valid expression for $K_{i j}$. The second advantage lies in the ability to make use of results for analogous effects from single-material turiulence theory. The following section reviews some of the important results from single-fluid turbulence theory that will be utilized by analogy in the developments of later chapters.

\section{PHYSICAL APPROACH TO SINGLE-FLUID TURBULENCE}

This section contains a discussion of what may be called the physical approach to turbulence theory. The goal here is to first establish some of the physical features of turbulent fluid flows that are commonly used as guidance in theoretical developments. Then a description of the most common predictive methods that exist for single-fluid turbulent flows can be presented. This provides a background knowledge useful in the developments of later chapters.

A variety of texts exist in which the broader historical developments in turbulence theory are traced. The book by Beran (1968) is one example where both the physical approach and the more mathematical approaches using statistical methods and spectral dynamics are described. Other useful sources are books by Tennekes and Lumley (1972), Bradshaw (1976), and ifinze (1975 2d ed).

The physical approach to turbulence theory combines a few essential elements of analysis with a great deal of physical reasoning, in the attempt to find a general description of turbulent flow behavior. In this process, it is usually necessary to depend on experimental data to complete the formulation of dynamical equations. Thus, the product is a semi-empirical theory, the most popular of which is called the $k-\epsilon$ model, which is due to a large number of contributors. Details of the development, and a description of the $k-\epsilon$ physical theory for turbulence are provided in this section.

The essential physical features of single fluid turbulent flows are the following:

1. Turbulent flows contain velocity and pressure fluctuations that have

a significant influence on the mean flow behavior.

2. Turbulent flows are diffusive of mean flow momentum.

3. Turbulent flows occur at large Reynolds numbers. 
4. Turbulent flows are three dimensional, rotational, anisotropic and are usually statistically nonhomogeneous.

5. Turbulen^ flows are dissipative of mean flow kinetic energy.

The following paragraphs contain a discussion of how most of these basic features have been established by straightforward analysis of the fluid conservation laws.

The first feature of turbulent flows follows by the definition given in Sec. 1. This is mentioned here in order to emphasize the point that turbulence is a matter of observational perspective. One nbserver's chaos can be another observer's perfect order. The remaining features of turbulent flows are established by considering the fluid conservation equations.

Most of the progress in turbulence theory has been made for the case of a single incompressible fluid. The essential first assumption made in most studies is that the eritire dynamical behavior of such a fluid is described by the instantaneous equations of motion.

These equations are the mass conservation equation

$$
\frac{\partial u_{j}}{\partial x_{j}}=0,
$$

and the momentum conservation equation

$$
\frac{\partial u_{i}}{\partial t}+\frac{\partial}{\partial x_{j}} u_{i} u_{j}=-\frac{1}{\rho} \frac{\partial p}{\partial x_{i}}+\frac{1}{\rho} \frac{\partial \tau_{i j}}{\partial x_{i}},
$$

in which $\mathbf{u}(\mathbf{x}, t)$ is the velocity, $p$ is the pressure, $\rho$ is the constant fiuid density and

$$
\tau_{i j}=\mu\left(\frac{\partial u_{i}}{\partial x_{j}}+\frac{\partial u_{j}}{\partial z_{i}}\right)
$$

is the fluid viscous stress tensor, with viscosity $\mu$. The average behavior is obtained by so-called Reynolds decomposition and averaging Eqs. (1.23) and (1.24). This involves describing the fluid velocity and pressure as the sum of mean and fluctuating parts, defined such that the fluctuating part has a zero mean value. The average is defined as a time average, or equivalently an ensemble average, and will be denoted here by an overbar. The instantaneous velocity is written $u_{i}=\bar{u}_{i}+u_{i}^{\prime}$ and the instanteneous pressure is written $p=\bar{p}+p^{\prime}$, where the prime denotes the fluctuating quantity. By definition $\overline{u_{i}^{\prime}}=0$ and $\overline{p^{\prime}}=0$, but in general $\overline{u_{i}^{\prime} u_{j}^{\prime}}$ and $\overline{u_{i}^{\prime} p^{\prime}}$ are nonzero.

Decomposing Eq.(1.23) and averaging yields two results. These are

$$
\frac{\partial \bar{u}_{j}}{\partial x_{j}}=0
$$

and

$$
\frac{\partial u_{j}^{\prime}}{\partial x_{j}}=0
$$


so the mean flow velocity and the fluctuations in velocity both satisfy an incompressibility condition. Decomposing Eq. (1.24) and averaging yields the mean momentum equation

$$
\frac{\partial \bar{u}_{i}}{\partial t}+\frac{\partial}{\partial x_{j}} \bar{u}_{i} \bar{u}_{j}=-\frac{1}{\rho} \frac{\partial \bar{p}}{\partial x_{i}}+\frac{1}{\rho} \frac{\partial \bar{\tau}_{i j}}{\partial x_{j}}-\frac{\partial}{\partial x_{j}} \overline{u_{i}^{\prime} u_{j}^{\prime}},
$$

where

$$
\bar{\tau}_{i j}=\mu\left(\frac{\partial \bar{u}_{i}}{\partial x_{j}}+\frac{\partial \bar{u}_{j}}{\partial x_{i}}\right)
$$

The last term in (1.28) contains the well-known Reynolds stress per unit mass

$$
R_{i j}=-\overline{u_{i}^{\prime} u_{j}^{\prime}},
$$

which is a result of averaging the advection term in Eq. (1.24). The matter of connecting the Reynolds stress to other mean flow quantities is the essence of turbulence theory.

Equation (1.30) establishes the second main feature of turbulent flows. The Reynolds stress is a flux of fluctuational momentum per unit mass carried by the fluctuating velocity. When velocity deviations from the mean (flurtuations) are negligible, the Reynolds stress is negligible. Thus non-negligible deviations from the mean velocity introduce a diffusion in mean momentum. This is analogous to the source of momentum diffusion in the laminar flow of a gas. In that case, a molecular viscosity arises if the distribution in molecular velocities departs significantly from the equilibrium Maxwell-Boltzmann distribution function (Vincenti and Kruger 1965).

The third, fourth, and fifth features of turbulent flows are established by consideration of the distribution in kinetic energy in a turbulent flow. The total kinetic energy per unit mass at a point in the flow is $\frac{1}{2} u_{i} u_{i}$. Its conservation equation is obtained by multiplying the instantaneous momentum equation, Eq. (1.24), by $u_{i}$. The mean flow kinetic energy is $\frac{1}{2} \bar{u}_{i} \bar{u}_{i}$ whose conservation equation is $\bar{u}_{i}$ times Eq. (1.28). The kinetic energy per unit mass contained in the fluctuations is $\frac{1}{2}\left(u_{i} u_{i}-\bar{u}_{i} \bar{u}_{i}\right)$ whose average is usually given the symbol $k$,

$$
k \equiv \frac{1}{2} \overline{u_{i}^{\prime} u_{i}^{\prime}}
$$

The equation for $k$ is the difference between the equation for $\frac{1}{2} u_{i} u_{i}$ and $\frac{i}{2} \bar{u}_{i} \bar{u}_{i}$, averaged. This is

$$
\begin{aligned}
\frac{\partial k}{\partial t}+\frac{\partial}{\partial x_{j}} \bar{u}_{j} k= & -\frac{1}{\rho} \frac{\partial}{\partial x_{j}} \overline{u_{j}^{\prime} p^{\prime}} \\
& -\frac{1}{\rho} \frac{\partial}{\partial x_{j}} \frac{1}{2} \overline{u_{i}^{\prime} u_{i}^{\prime} u_{j}^{\prime}}
\end{aligned}
$$




$$
\begin{aligned}
& -\frac{1}{\rho} \frac{\partial}{\partial x_{j}} \overline{u_{i}^{\prime} \tau_{i j}^{\prime}} \\
& +R_{i j} \frac{\partial \bar{u}_{i}}{\partial x_{j}} \\
& -\frac{1}{\rho} \overline{\tau_{i j}^{\prime} \frac{\partial u_{i}^{\prime}}{\partial x_{j}}}
\end{aligned}
$$

where

$$
\tau_{i j}^{\prime}=\mu\left(\frac{\partial u_{i}^{\prime}}{\partial x_{j}}+\frac{\partial u_{j}^{\prime}}{\partial x_{i}}\right) .
$$

Because the average of the fluctuational kinetic energy $k$ is equal to $-\frac{1}{2} R_{i i}$, $k$ is considered to be a relevant measure of the significance of the turbulence itself. Therefore Eq. (1.31) can be used to examine the sources and sinks for the turbulence. The first three terms on the right of Eq. (1.31) are conservative, because the density $\rho$ is a constant. This means that terms (a), (b), and (c) serve to redistribute the content of $k$ among the elements of fluid contained in a given control volume. Term (a) is called the pressure work term, term (b) is called the energy diffusion term and term (c) is called the viscous work term (Hinze 1975). Term (d) in Eq. (1.31) is called the kinetic energy creation term because the negative of this term appears in the conservation equation for $\frac{1}{2} \bar{u}_{i} \bar{u}_{i}$ (Tennekes and Lumley 1972). This means that term (d) measures the rate at which mean flow kinetic energy (ordered) is transferred into the kinetic energy of the velocity fluctuations (disordered).

Term (e) is called the dissipation term (Tennekes and Lumley 1972). This is because it can be written

$$
\begin{aligned}
-\frac{1}{\rho} \overline{\tau_{i j}^{\prime} \frac{\partial u_{i}^{\prime}}{\partial x_{j}}} & =-\nu \overline{\left(\frac{\partial u_{i}^{\prime}}{\partial x_{j}}+\frac{\partial u_{j}^{\prime}}{\partial x_{i}}\right) \frac{\partial u_{j}^{\prime}}{\partial x_{i}}} \\
& =-\frac{\nu}{2} \overline{\left(\frac{\partial u_{i}^{\prime}}{\partial x_{j}}+\frac{\partial u_{j}^{\prime}}{\partial x_{i}}\right)^{2}} \\
& =-\epsilon,
\end{aligned}
$$

because the tensor $\tau_{i j}^{\prime}$ is symmetric, and where $\nu$ is the kinematic viscosity. This quantity is so important to the physical theory of turbulence that it is referred to almost universally by the symbol $\epsilon$. Clearly $\epsilon$ is a positive quantity containing the fluid viscosity. The term $-\epsilon$ measures the rate at which the kinetic energy in the turbulence is dissipated into heat.

This establishes the fifth feature of turbulent flows. Anytime $R_{i j}$ is large enough to cause significant diffusion of mean momentum, mean flow gradients 
cause the transfer of mean flow kinetic energy into $k$, which is a measure of $R_{i j}$. This transfer is accompanied by viscous dissipation into heat.

The fuurth feature of turbulent flows is more easily established by considering the equation of change for $R_{i j}$ itself, rather than Eq. (1.31). Hinze (1975), for example, uses the $R_{i j}$ equation in the case of a simple shear flow to demonstrate that the pressure work term causes a redistribution of the kinetic energy among the coordinate components of velocity causing rotation, anisotropy and three dimensionality.

The third feature of turbulent flows is now plain to see. In order to create turbulence, the rate of dissipation must be smaller than the transfer rate of energy from the mean flow. The energy transfer rate has its roots in the nonlinear advection term of Eq. (1.24). The dissipation term has its roots in the viscous term of Eq. (1.24). The competition between viscous momentum transport and inertial transport is measured by the Re,/nolds number $R e$ defined by

$$
R e=\frac{U d}{\nu}
$$

where $U$ is a characteristic average flow speed and $d$ is a characteristic length dimension in the flow system. A large Reynolds number means that the nonlinear effects tending to create the turbulence dominate over the viscous effects that cause it to disappear. Thus the higher the Reynolds number, the higher is the propensity for the flow to become turbulent.

So far only the qualitative features of single fluid turbulent flows have been discussed. The following paragraphs contain a brief discussion of some quantitative aspects of turbulence theory that will be useful in later developments.

Equations (1.28) and (1.31) exhibit an essential problem in quantitative turbulence theory. The Reynolds stress $R_{i j}$ appears in Eq. (1.28) for the mean momentum. Consider relating $R_{i j}$ to the average energy $k$ obtained from Eq. (1.31) which contains higher-order averages such as $\frac{1}{2} \overline{u_{i}^{\prime} u_{i}^{\prime} u_{j}^{\prime}}$ and $\epsilon$. Equations for these average products contain even higher order average products, so the process of deriving transport equations for averages of fluctuating quantities can never yield a closed set of equations. This is the so-called closure problem. A variety of workers have combined physical arguments, dimensional analysis and experimental data to approach the closure problem. An important example is the $k-\epsilon$ approach, which will be described here because of its widespread application to turbulent flows occurring in engineering (Reynolds and Cebeci 1976).

The $k-\epsilon$ turbulence theory represents the present status in the evolution of practical methods for predicting the behavior of complex turbulent flows (Launder and Spalding 1974). Several workers have contributed to the development of the $k-\epsilon$ theory. This developmental process has spanned about the past 100 years, beginning with Reynold's work in the 1880's.

In the late 1800's, following Reynolds' pioneering work, Boussinesq established the first key element of the $k-\epsilon$ theory by introducing the idea of turbulent 
viscosity (Beran 1968). The main idea of Boussinesq was to find a coefficient $\nu_{t}$ from experiments, that could be used to represent the Reynolds stress in a way analogous to the stress due to molecular viscosity. Thus Boussinesq proposed to represent $R_{i j}$ by

$$
R_{i j}=\nu_{t}\left(\frac{\partial \bar{u}_{i}}{\partial x_{j}}+\frac{\partial \bar{u}_{j}}{\partial x_{i}}\right)
$$

which can be thought of as a definition of the turbulent viscosity $\nu_{t}$ (also called the eddy viscosity). The diagonal parts of Eq. (1.35) are zero, because of Eq. (1.26), so that one must think of the fluid pressure as containing the diagonal part of $R_{i j}$. A generalized form of Eq. (1.35), which has become the modern definition of $\nu_{t}$, is

$$
R_{i j}=-\frac{2}{3} k \delta_{i j}+\nu_{t}\left(\frac{\partial \bar{u}_{i}}{\partial x_{j}}+\frac{\partial \bar{u}_{j}}{\partial x_{i}}\right)
$$

This can be thought of as keeping the turbulence pressure separate from the mean pressure.

In the mid-1920's Prandtl provided an intermediate step in the development of $k-\epsilon$ theory by introducing the famous mixing length hypothesis (Beran 1968). In this, $\nu_{t}$ is considered to be proportional to

$$
\left|\frac{\partial \bar{u}_{i}}{\partial x_{j}}\right|
$$

scaled by a distance $l_{m}$ called the mixing length. For flow in a pipe, Prandtl supposed the mixing length to be a function of distance from the wall, where $l_{m}$ is zero. This way of thinking is useful but has proven to be of value mainly for simple shear flows (Reynolds and Cebeci 1976).

The next essential step in the $k-\epsilon$ development was taken in the mid-1940's. This step involves connection of $\nu_{t}$ to $k$ by the so-called Kolmogorov-Prandtl formula (Rodi 1980)

$$
\nu_{t}=C_{\mu}^{\prime} k^{\frac{1}{2} l}
$$

where $C_{\mu}^{\prime}$ is an empirical coefficient and $l$ is the integral scale of the turbulence. The integral scale is the separation distance over which the velocity at one point in the flow remains statistically dependent on the velocity at another point in the flow.

By the mid-1960's workers began to suspect that Eq. (1.37) might be valuable if one could obtain $l$ from an evolution equation that accounted for the physical increase and decrease in $l$ with the flow. The first systematic attempt at developing an equation for $l$ was made by Harlow and Nakayama (1967). Shortly after that these same workers realized that one could profit by working instead with an 
equation for the dissipation rate (Harlow and Nakayama 1968). The next essential step is the realization that $\epsilon$ can be related to $l$ by

$$
\epsilon=\frac{C_{D} k^{3 / 2}}{l}
$$

Equation (1.38) follows by way of dimensional considerations, so that $C_{D}$ is a coefficient expected to be near unity. Note that Eq. (1.38) is free of the molecular viscosity $\nu$. Expression (1.38) for $\epsilon$ can be thought of as representing the rate at which turbulence energy is transferred from large scale structures in the fluid to successively smaller ones. In a time-steady flow this transfer rate must be equal to the dissipation rate.

Combining Eqs. (1.37) and (1.38) yields

$$
\nu_{t}=\frac{C_{\mu} k^{2}}{\epsilon}
$$

where $C_{D} C_{\mu}^{\prime}$ is replaced by $C_{\mu}$. Now the task is to develop a model equation for $\epsilon$ instead of an equation for the integral scale $l$. An important clue for finding the required model equation is provided by considering the exact equation for the isotropic dissipation rate $D$, where

$$
D=\nu \overline{\frac{\partial u_{i}^{\prime}}{\partial x_{l}} \frac{\partial u_{i}^{\prime}}{\partial x_{l}}}
$$

One can expect $\epsilon$ to behave generally like $D$. The equation for $D$ is obtained by multiplying the equation for $u_{i}^{\prime}=u_{i}-\bar{u}_{i}$ by the operator

$$
2 \nu \frac{\partial u_{i}^{\prime}}{\partial x_{l}} \frac{\partial}{\partial x_{l}}
$$

and averaging (Harlow and Nakayama 1968). The result is

$$
\begin{aligned}
\frac{\partial D}{\partial t}+\bar{u}_{j} \frac{\partial D}{\partial x_{j}}= & -2 \nu \frac{\partial}{\partial x_{j}}\left(\overline{\left.\frac{1}{\rho} \overline{\frac{\partial p^{\prime}}{\partial x_{l}} \frac{\partial u_{j}^{\prime}}{\partial x_{l}}}\right)}\right. \\
& -\nu \frac{\partial}{\partial x_{j}} \overline{\left(u_{j}^{\prime} \frac{\partial u_{i}^{\prime}}{\partial x_{l}} \frac{\partial u_{i}^{\prime}}{\partial x_{l}}\right)} \\
& +\nu \frac{\partial}{\partial x_{j}}\left(\frac{\partial D}{\partial x_{j}}\right) \\
& -2 \nu \frac{\partial^{2} \bar{u}_{i}}{\partial x_{j} \partial x_{l}}\left(\overline{u_{j}^{\prime} \frac{\partial u_{i}^{\prime}}{\partial x_{l}}}\right)
\end{aligned}
$$




$$
\begin{aligned}
& -2 \nu\left[\frac{\partial \bar{u}_{i}}{\partial x_{j}} \overline{\left(\frac{\partial u_{i}^{\prime}}{\partial x_{l}} \frac{\partial u_{j}^{\prime}}{\partial x_{l}}\right)}+\frac{\partial \bar{u}_{j}}{\partial x_{l}}\left(\overline{\frac{\partial u_{i}^{\prime}}{\partial x_{l}} \frac{\partial u_{j}^{\prime}}{\partial x_{l}}}\right)\right] \\
& -2 \nu \overline{\frac{\partial u_{i}^{\prime}}{\partial x_{l}} \frac{\partial u_{i}^{\prime}}{\partial x_{j}} \frac{\partial u_{j}^{\prime}}{\partial x_{l}}} \\
& -2 \nu^{2} \overline{\frac{\partial^{2} u_{i}^{\prime}}{\partial x_{j} \partial x_{l}} \frac{\partial^{2} u_{i}^{\prime}}{\partial x_{j} \partial x_{l}}} .
\end{aligned}
$$

The first three terms on the right of Eq. (1.41) are conservative because $\nu$ is constant. These terms quantify the way that $D$ is changed by diffusive processes. Terms (d), (e), and (f) are analogous to the creation term in Eq. (1.31) for $k$, and are therefore called creation terms. Term $(\mathrm{g})$ is analogous to $\epsilon$ and is therefore called the destruction term (Reynolds and Cebeci 1976). Hence $D$, and consequently $\epsilon$, is seen to evolve in a way that depends upon the competition among the effects of diffusion, creation and destruction. Note that Eq. (1.31) for $k$ shows the same surt of competing effects for the turbulence energy.

The final and current stage of $k-\epsilon$ theory development involves estimation of the exact diffusion terms in Eq. (1.31) and postulating an equation for $\epsilon$ knowing the general behavior of $D$.

A variety of workers have proposed to represent the diffusive flux of turbulence energy by a diffusive-like term (Harlow and Nakayama 1967, Launder and Spalding 1974). One form is

$$
\frac{1}{2} \overline{u_{i}^{\prime} u_{i}^{\prime} u_{j}^{\prime}}+\frac{1}{\rho} \overline{u_{j}^{\prime} p^{\prime}}=\frac{\nu_{t}}{\sigma_{k}} \frac{\partial k}{\partial x_{j}},
$$

where $\sigma_{k}$ is a dimensionless coefficient. The coefficient $\sigma_{k}$ is the ratio of momentum diffusion by the turbulence to the turbulent diffusion of turbulence kinetic energy. This can be thought of as a turbulent Prandtl number. Combining Eqs. (1.42), (1.36), and (1.33) with Eq. (1.31) yields a modeled form of the $k$ equation,

$$
\frac{\partial k}{\partial t}+\frac{\partial}{\partial x_{j}} u_{j} k=\frac{\partial}{\partial x_{j}}\left(\frac{\nu_{t}}{\sigma_{k}} \frac{\partial k}{\partial x_{j}}\right)+P-\epsilon
$$

where

$$
P=R_{i j} \frac{\partial \bar{u}_{i}}{\partial x_{j}}
$$

is the turbulence energy production rate, in which $R_{i j}$ is given by Eq. (1.36).

By analogy to Eq. (1.43) Hanjalić and Launder (1972) postulated that a good estimate of $\epsilon$ can be obtained by solving

$$
\frac{\partial \epsilon}{\partial t}+\frac{\partial}{\partial x_{j}} u_{j} \epsilon=\frac{\partial}{\partial x_{j}}\left(\frac{\nu_{t}}{\sigma_{\epsilon}} \frac{\partial \epsilon}{\partial x_{j}}\right)+\frac{\epsilon}{k}\left(C_{1} P-C_{2} \epsilon\right),
$$


in which $\sigma_{\epsilon}$ is an empirical coefficient and $C_{1}$ and $C_{2}$ are coefficients of order unity. The first term on the right of Eq. (1.45) is a diffusion term written in the same spirit as Eq. (1.42) for the turbulent diffusion of $k$. The second and third terms of Eq. (1.45) are designed to represent the production and destruction of $\epsilon$, by dimensional analogy to the corresponding terms in Eq. (1.43). The task of establishing values for the coefficients in Eqs. (1.45), (1.43), and (1.39) has mainly been performed by Launder and Spalding (1974), who report

$$
C_{\mu}=0.09, \quad \sigma_{k}=1.0, \quad \sigma_{\epsilon}=1.3, \quad C_{1}=1.44 \text {, and } C_{2}=1.92 \text {, }
$$

for turbulent flow remote from walls. Equations (1.46), (1.45), (1.43), (1.39), and (1.36) are the $k-\epsilon$ turbulence model for flow at high Reynolds numbers. This model has proven accurate for a variety of flow circumstances including pipe flow, jets and wakes. Unfortunately, the optimum values for the coefficients do not appear to be universal (Launder and Spalding 1974). This means that the model cannot in general be expected to yield accurate results in flow situations far different from the experimental conditions by which the model is calibrated. Nevertheless a sufficient number of industrial circumstances exists for which the $k-c$ model is quite accurate, making the present state of the theory very useful.

The foregoing representation of the Reynolds stress belongs to what is called the two-equation class of turbulence models (Reynolds 1976). This is because the formulation requires transport equations for the two auxiliary quantities $k$ and $\epsilon$, on which $R_{i j}$ is assumed to depend. Two-equation models, such as the $k-\epsilon$ model just described, are considered to be a practical alternative to the much more complex Reynolds stress transport approach. In the latter method, one provides transport equations for each of the six unique elements of the tensor $R_{i j}$, in addition to, say, an equation for $\epsilon$. Two-equation models are also considered to be of more general value than one-equation ( $k$ only) or zero equation (mixing length) models (Reynolds and Cebeci 1976).

One undesirable feature of nearly all physically based quantitative turbulence theory is that empirical data is still required for obtaining closed equations. Nonetheless the values of the coefficients given in the $k-\epsilon$ model are demonstrated to give fairly accurate predictions on a wide class of turbulent flows (Rodi 1980). This means that, despite the empiricism, the emerging theory is beginning to show signs of a universal behavior.

The approach to multimaterial flow theory in this thesis is also physically based, so that some empiricism is still required. The plan is to draw heavily on the analogies that will be shown to exist between multimaterial flows and singlefluid turbulent flows. These analogies will be established by showing that these two circumstances have similar features, both physical and mathematical. The hope is to bring multimaterial flow theory forward to the point where a general set of equations is capable of describing a broad class of flow behavior. The next section provides an overview of the manner in which this goal is pursued in the remaining chapters. 


\section{OVERVIEW OF THE MULTIMATERIAL STATISTICAL THEORY}

This section is a brief overview of the developments that follow in the remair ing chapters. The goal here is to provide an idea of how the thesis is organized. The organization is designed in such a way that the effects of turbulence on the mean flow are considered separately from the multimaterial effects. After establishing the multimaterial and turbulence effects separately, the combined influence on the mean flow behavior is developed.

Chapter II provides the statistical basis upon which the remainder of the theory is built. The purpose of Chapter II is to establish the dependence of the mean flow on certain probability distribution functions. Development of the mean equations of motion requires the definition of ensemble average distribution functions for the velocity at a point in the flow, at any instant. Because multimaterial cases are the subject, the phase space of the distribution functions must contain an independent variable that relates to the probability of finding a given material at a point. In Chapter II the average distribution functions needed for the multimaterial statistical theory are defined. Using the well-known instantaneous equations of motion at a point in pure material, the average equations are derived.

The average conservation equations for mass, momentum, and fluctuational kinetic energy derived in Chapter II are unclosed in the usual sense that they contain averages of products in the fluctuating velocity. These average products, or correlations, are expressed in terms of integrals over the probability distribution functions. The value in expressing correlations in this way emerges in Chaptirs III and IV. However, even at the unclosed stage of Chapter II, one can see the separation of terms due to fluctuations from those that arise from the finite probability of finding any one of, say, two materials at a point. One of the main results of Chapter II is the definition of the multimaterial analog of the Reynolds stress. The form of the multimaterial Reynolds stress and the form of the multimaterial equation for mean fluctuational kinetic energy demonstrate a strong parallel between the multiphase case and the case of a single turbulent fluid. This suggests that closure of the multimaterial equations can be accomplished in a way that benefits from existing theory for single-fluid turbulence.

The closure problem is approached in stages. In Chapter III a Gaussian form for the distribution in velocity at a point is postulated. This corresponds to the case of local equilibrium in molecular gas dynamics, which leads to nonviscous flow theory. In the case of incompressible turbulent mixtures this postulate leads to what may be called a nonviscous theory, in the sense of the turbulent viscosity. That is to say, the off-diagonal parts of the multimaterial Reynolds stress, and the diffusive terms in the turbulence kinetic energy equation, are all zero. This is not to say that the effects of the turbulent viscosity are unimportant. The main goal of Chapter III is to focus attention on the effects of the mean flow due to the presence of more than one material.

In Chapter III the objective is to obtain basic understanding of the statistical 
nature of the mean flow, as influenced by the interpenetrating motion of materials. This is mainly accomplished by examining the nature of terms containing the multipoint distribution functions in the mean flow equations. Closure is obtained for these terms by postulating physically realistic forms for the multipoint correlations that arise from the multipoint distribution functions. This is done for the case of a locally homogeneous statistical field. The goa! of Chapter IV is to extend the results of Chapter III to the general case of a nonhomogeneous statistical field. For the multimaterial Reynolds stress, the multimaterial analog of the $k-\epsilon$ theory is developed. This is shown to be related to the more fundamental theory of nonhomogeneous turbulence due to Lundgren $(1967,1969)$. The nonhomogeneous nature of the momentum exchange terms is then introduced in a fashion consistent with the multimaterial $k-\epsilon$ theory.

In Chapter $\mathrm{V}$ a numerical method is presented for solution of the system of partial differential equations developed in the earlier chapters. Here the computer code that carries out the solution is also described. Chapter VI contains a set of computations designed to demonstrate the application of the multimaterial statistical theory to problems of interest in engineering. The final chapter summarizes the results of the thesis and discusses some of the directions that can be pursued as a consequence of this work.

\section{References Cited}

Anderson, T. B. and R. Jackson. 1967. A fluid mechanical description of fluidized beds. Ind. and Eng. Chem. Fund. 6:527-639.

Batchelor, G. K. 1967. An introduction to fluid dynamics. Cambridge: Cambridge University Press.

Bear, J. 1972. Dynamics of fluids in porous media. New York: American Elsevier. Beran, M. J. 1968. Statistical continuum theories. New York: Interscience Publishers.

Besnard, D. and F. H. Harlow. 1985. Turbulence in two-field incompressible flow. Los Alamos National Laboratory report LA-10187-MS.

Bradshaw, P. 1976. Turbulence. Berlin: Springer-Verlag.

Buyevich, Y. A. 1971. Statistical hydromechanics of disperse systems. (3 parts). J. Fluid Mech. 49:489-507, 52:345-355, 56:313-336.

Chandrasekhar, S. 1943. Stochastic problems in physics and astronomy. Rev. Modern Phys. 15:1-89.

Crowe, C. T. 1982. Numerical models for dilute gas-particle flows. J. Fluids Eng. 104:297-303. 
Crowe, C. T. and D. T. Pratt. 1972. Two-dimensional gas-particle flows. In R. B. Landis and G. J. Hordemann, eds. Proceedings of the 1972 heat transfer and fluid mechanics institute. Stanford: Stanford University Press.

Currie, I. G. 1974. Fundamental mechanics of fluids. Toronto: McGraw-Hill.

Drew, D. A. 1971. Averaged field equations for two-phase media. Studies in Applied Math. 50:133-166.

Dukowicz, J. K. 1980. A particle-fluid numerical model for liquid sprays. $J$. Comput. Phye. 35:229-253.

Elghobashi, S. E. and T. W. Abou-Arab. 1983. A two-equation turbulence model for two-phase flows. Phys. Fluids. 26:931-938.

Fan, L. T., S. H. Hwang, S. T. Chou and R. Nassar. 1985. Birth-death modeling of deep bed filtration: sectional analysis. Chem. Eng. Commun. 35:101-121.

Hanjalić, K. and B. E. Launder. 1972. A Reynolds stress model of turbulence and its application to thin shear flows. J. Fluid Mech. 52:689-XXX.

Harlow, F. H. and A. A. Amsden. 1975. Numerical calculation of multiphase fluid flow. J. Comput. Phys. 17:19-52.

Harlow, F. H. and P. I. Nakayama. 1967. Turbulence transport equations. Phys. Fluids. 10:2323-2332.

. 1968. Transport of turbulence energy decay rate. Los Alamos Scientific Laboratory report LA-3854.

Hetsroni, G. 1982. ed. Handbook of multiphase systems. New York: McGraw-Hill. Hinze, J. O. 1975. 2d ed. Turbulence. New York: McGraw-Hill.

Ishii, M. 1975. Thermo-fluid dynamic theory of two-phase flow. Eyrolles: Direction des Études et Recherches d'Électricité de France.

Launder, B. E. and D. B. Spalding. 1974. The numerical computation of turbulent flows. Comp. Meth. App. Mech. Eng. 3:269-289.

Lourenco, L. M., M. L. Riethmuller and J. A. Essers. 1983. A kinetic approach to gas-solid suspensions. in J. T. Jurewicz, ed. gas-solid flows. New York: ASME.

Lundgren, T. S. 1967. Distribution functions in the statistical theory of turbulence. Phys. Fluids. 10:969-975.

. 1969. Model equation for nonhomogeneous turbulence. Phys. Fluids. 12:485-497.

1972. Slow flow through stationary random beds and suspensions of spheres. J. Fluid Mech. 51:273-299.

Maxey, M. R. and J. J. Riley. 1983. Equation of motion for a small rigid sphere in a nonuniform flow. Phys. Fluids. 26:883-889. 
Murray, J. D. 1965. On the mathematics of fluidization. Part 1. Fundamental equations and wave propagation. J. Fluid Mech. 21:465-493.

Needham, D. J. and J. H. Merkin. 1983. The propagation of a voidage disturbance in a uniformly fluidized bed. J. Fluid Mech. 131:427-454.

Oates, G. C. 1974. Vector Analysis. in C. E. Pearson, ed. Handbook of applied mathematics. New York: van Nostrand Reinhold.

O'Rourke, P. J. 1981. Collective drop effects on vaporizing liquid sprays. Doctoral dissertation, Princeton University. Also, Los Alamos National Laboratory report LA-9069-T.

Reynolds, W. C. and T. Cebeci. 1976. Calculation of turbulent flows. in P. Bradshaw ed. Turbulence. Berlin: Springer-Verlag.

Rodi, W. 1980. Turbulence models and their application in hydraulics. The Netherlands: International Assoication for Hydraulic Research.

Tennekes, H. and J. L. Lumley. 1972. A first course in turbulence. Cambridge: The MIT Press.

Travis, J. R., F. H. Harlow, and A. A. Amsden. 1976. Numerical calculation of two-phase flows. Nuc. Sci. Eng. 61:1-10.

Westbrook, C. K. 1977. Three-dimensional numerical modeling of liquid fuel sprays. The sixteenth symposium on combustion. Pittsburgh: The Combustion Institute.

Whitaker, S. 1973. The transport equations for multi-phase systems. Chem. Eng. Sci. 28:139-147.

Williams, F. A. 1985. 2d ed. Combustion theory. Menlo Park: BenjaminCummings. 


\section{Chapter II \\ Statistical Basics}

The goal of this chapter is to establish the statistical basis for development of a general theory for incompressible, turbulent multimaterial flow. The approach used here parallels and extends the method introduced by Lundgren (1967) for a single turbulent incompressible fluid.

A general theory provides the necessary connection between the local, instantaneous flow and the mean flow. The main purpose of this chapter is to develop the conservation equations for the mean quantities of interest on a statistical basis. These equations, although comprising an unclosed set, provide the starting point for development of the theory. Closure is approached in a series of stages that are presented in succeeding chapters.

For simplicity the case of two immiscible, incompressible fluids is first considered here. Later in this chapter the case of rigid, nonfragmenting bodies, suspended in an incompressible fluid, is shown to be a subset of the two-fluid case.

The first step is to introduce a way to distinguish which of the two fluids is at a point, at any instant. Next, the ensemble average distribution functions for material and velocity at a point are defined. Then the instantaneous equation of motion for an ordinary point is introduced, and the pressure is eliminated using the incompressibility condition. The foregoing steps lead directly to the average conservation equations for mass, momentum, and fluctuational kinetic energy of each of the two fluids. These equations are unclosed in the sense that they contain integrals over the phase space of the still unknown distribution functions. In later chapters the closure problem is approached by estimating the effects of the integral terms in a set of successively higher approximations.

\section{DISTRIBUTION FUNCTIONS IN THE MULTIMATERIAL THEORY}

To begin, a method is needed for identifying which fluid is at a point in space $\mathbf{x}$, at time $t$. This is provided by introducing a set of "material functions" $\theta^{(k)}(\mathbf{x}, t)$ for materials $k=1,2$ defined such that $\theta^{(k)}$ has a unit value if material $k$ is at the point $(x, t)$, and zero otherwise. Hence at any point in space, and at any instant

$$
\theta^{(1)}+\theta^{(2)}=1 .
$$

By definition the material functions $\theta^{(k)}$ are constants along the motion of the fluid, therefore they satisfy the equation

$$
\frac{\partial \theta^{(k)}}{\partial t}+u_{j} \frac{\partial \theta^{(k)}}{\partial x_{j}}=0
$$


where $\mathbf{u}(x, t)$ is the velocity at $(\mathbf{x}, t)$. (As in Chapter $\mathrm{I}$, the superscript in parenthesis is used to denote a quantity associated with a particular fluid.)

The ensemble average distribution function $f\left(\theta^{(k)}, \mathbf{u}\right)$ is defined such that $\int d \theta^{(k)} d u$ is the joint probability that the material function is in $\theta^{(k)}+d \theta^{(k)}$ and that the velocity is in $\mathbf{u}+d \mathbf{u}$. Clearly $f\left(\theta^{(k)}, \mathbf{u}\right)$ has a zero value for all $\theta^{(k)}$ intervals except the two end intervals containing the points $\theta^{(k)}=1$ and $\theta^{(k)}=0$. It is helpful to visualize a particular case of $f$. Consider a specific situation in which the horizontal component of velocity $u$ is randomly distributed with zero mean for both materials. The view of $f$ for this circumstance is sketched in Fig. 1 .

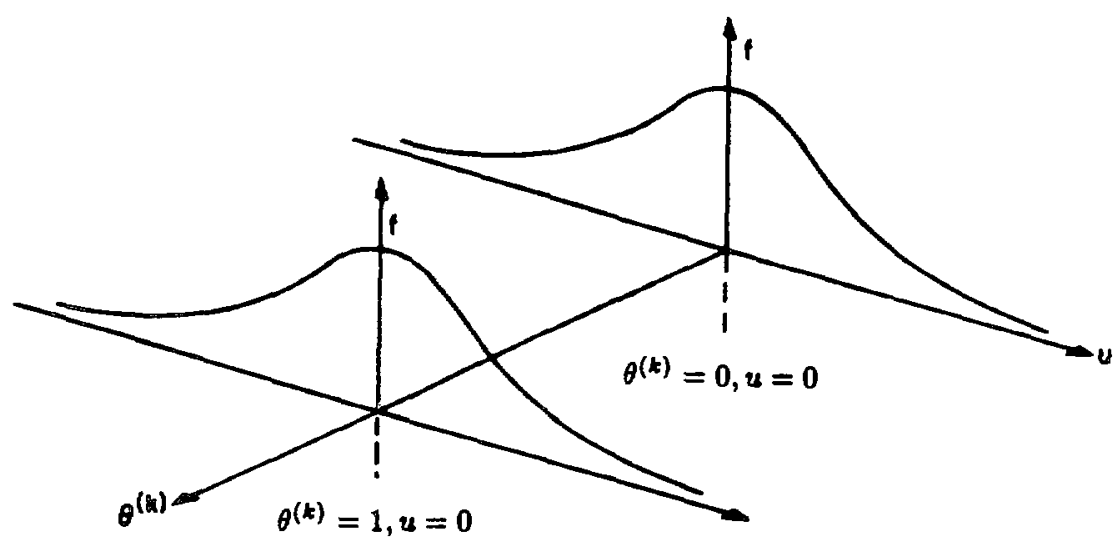

Figure 1. Sketch of a particular realization of the distribution function $f$.

The expectation value of any quantity dependent on $\theta^{(k)}$ and/or $u$ is found by multiplying the quantity by $f$ and integrating over all possible values of $\theta^{(k)}$ and $u$. In that which follows, all such integrations are denoted by the expectation sign \langle\rangle . For later convenience the independent variables are changed by writing

$$
\begin{aligned}
f^{(k)}(\mathbf{x}, \mathbf{v}, \alpha, t) & =\iint \delta\left(\theta^{(k)}-\alpha\right) \delta(\mathbf{u}-\mathbf{v}) f d \theta^{(k)} d \mathbf{u} \\
& =\left\langle\delta\left(\theta^{(k)}-\alpha\right) \delta(\mathbf{u}-\mathbf{v})\right\rangle
\end{aligned}
$$

where $\delta$ is again the Dirac delta function. Observe the identity

$$
\begin{aligned}
\iint f^{(k)} d \alpha d \mathbf{v} & =\iint\left\langle\delta\left(\theta^{(k)}-\alpha\right) \delta(\mathbf{u}-\mathbf{v})\right\rangle d \alpha d \mathbf{v} \\
& =\left\langle\iint \delta\left(\theta^{(k)}-\alpha\right) \delta(\mathbf{u}-\mathbf{v}) d \alpha d \mathbf{v}\right\rangle \\
& =1
\end{aligned}
$$


which gives the natural normalization of $f^{(k)}$.

The probability $f\left(\theta^{(k)}, \mathbf{u}\right)$ and consequently $f^{(k)}(\mathbf{x}, \mathbf{v}, \alpha, t)$ is defined in the ensemble average sense (Townsend $19762 \mathrm{~d}$ ed). That is to say, one imagines $N$ replicas of the same flow system, identical in all respects that one has taken care to measure. The replicas differ only with respect to say, initial conditions that have not been measured. In general the values of $\theta^{(k)}$ and $\mathbf{u}$ wili vary at any given point $(\mathbf{x}, t)$ among system replicas. The probability is the frequency with which the material function is observed in $\theta^{(k)}+d \theta^{(k)}$ and the velocity is observed in $\mathbf{u}+d \mathbf{u}$, in the limit $N \rightarrow \infty$.

In the manipulations that follow, it is frequently necessary to integrate over the phase space of the probability distribution functions. To make the equations more compact, the shorthand $d \mathbf{T}=d \alpha d \mathbf{v}$ is used for the element of volume in phase space of the single-point distribution function, not including position and time. With this the expected material function is

$$
\int \alpha f^{(k)} d \mathbf{\Gamma}=\left\langle\theta^{(k)}\right\rangle
$$

the expected momentum per unit mass is

$$
\int \alpha \mathbf{v} f^{(k)} d \mathbf{\Gamma}=\left\langle\theta^{(k)} \mathbf{u}\right\rangle
$$

and the expected momentum per unit mass, without regard to a specific material, is

$$
\int \mathbf{v} f^{(k)} d \mathbf{T}=\langle\mathbf{u}\rangle
$$

The quantity $\left\langle\theta^{(k)}\right\rangle$ is the expectation that material $k$ is at $(\mathbf{x}, t)$. In the case of two immiscible fluids, $0 \leq\left\langle\theta^{(k)}\right\rangle \leq 1$.

One can think of $\left\langle\hat{\theta}^{(k)}\right\rangle$ as the expected volume fraction of material $k$ at a point. To see this, integrate $\left\langle\theta^{(k)}\right\rangle$ over an arbitrary volume $V$ and divide by $V$. Thus

$$
\begin{aligned}
\frac{1}{V} \int_{V}\left\langle\theta^{(k)}(\mathbf{x}, t)\right\rangle d \mathbf{x} & =\frac{1}{V}\left\langle\int_{V} \theta^{(k)} d \mathbf{x}\right\rangle \\
& =\frac{\left\langle V^{(k)}\right\rangle}{V}
\end{aligned}
$$

where $\left\langle V^{(k)}\right\rangle$ is the expected volume within $V$ occupied by material $k$. Hence the mass and momentum densities, per unit total volume, are given by $\rho^{(k)}\left\langle\theta^{(k)}\right\rangle$ and $\rho^{(k)}\left\langle\theta^{(k)} \mathbf{u}\right\rangle$, where $\rho^{(k)}$ is the constant mass density of pure material $k$.

An important fact to note is that the material functions $\theta^{(k)}$ are discontinuous functions of space, at any instant. By definition $\nabla \theta^{(k)}=0$ everywhere except at an interface between the two fluids where it is infinite. This property of the material 
functions has been used in the literature as a way of separating the effects of a complicated (immeasurable) interfacial structure from the mean flow (measurable) effects (Lundgren 1972). This separation can be accomplished by arranging the equations in such a way that terms involving $\nabla \theta^{(k)}$ emerge. This procedure will be utilized in Sec. 3 of this chapter.

The quantity $\langle\mathbf{u}\rangle$ defined by Eq. (2.7) is the expected velocity at $(\mathbf{x}, t)$ without regard to which material is present. In the theory that follows the mean velocity of material $k$ at $(\mathrm{x}, t)$ is required. This is defined as the average momentum per unit average mass. Hence

$$
\left\langle\mathbf{u}^{(k)}\right\rangle=\frac{\left\langle\theta^{(k)} \mathbf{u}\right\rangle}{\left\langle\theta^{(k)}\right\rangle}
$$

is the average velocity of material $k$ at $(\mathbf{x}, t)$. With this, observe the relation

$$
\begin{aligned}
\langle\mathbf{u}\rangle & =\left\langle\theta^{(1)} \mathbf{u}+\theta^{(2)} \mathbf{u}\right\rangle \\
& =\left\langle\theta^{(1)}\right\rangle\left\langle\mathbf{u}^{(1)}\right\rangle+\left\langle\theta^{(2)}\right\rangle\left\langle\mathbf{u}^{(2)}\right\rangle,
\end{aligned}
$$

so that the velocity $\langle\mathbf{u}\rangle$ defined by Eq. $(2.7)$ will be called the "composite" velocity. Now the average fluctuational kinetic energy per unit mass is defined by

$$
\frac{1}{2} \int \alpha\left(v_{i}-\left\langle u_{i}^{(k)}\right\rangle\right)^{2} f^{(k)} d \Gamma=\left\langle\frac{1}{2} C_{i}^{(k) 2} \theta^{(k)}\right\rangle
$$

where $C_{i}^{(k)}=u_{i}-\left\langle u_{i}^{(k)}\right\rangle$.

The two-point probability distribution function is also needed. This is written

$$
\begin{aligned}
f_{1,2}^{(k, m)}\left(\mathbf{x}_{1}, \mathbf{v}_{1}, \alpha_{1}, \mathbf{x}_{2}, \mathbf{v}_{2}, \alpha_{2}, t\right) \\
=\left\langle\delta\left(\theta_{1}^{(k)}-\alpha_{1}\right) \delta\left(\mathbf{u}_{1}-\mathbf{v}_{1}\right) \delta\left(\theta_{2}^{(m)}-\alpha_{2}\right) \delta\left(\mathbf{u}_{2}-\mathbf{v}_{2}\right)\right\rangle
\end{aligned}
$$

and is defined such that $f_{1,2}^{(k, m)} d \alpha_{1} d v_{1} d \alpha_{2} d v_{2}=f_{1,2}^{(k, m)} d \Gamma_{1,2}$ is the joint probebility that $\alpha_{1}$ is in $\alpha_{1}+d \alpha_{1}, \alpha_{2}$ is in $\alpha_{2}+d \alpha_{2}, v_{1}$ is in $v_{1}+d v_{1}$, and $v_{2}$ is in $\mathrm{v}_{2}+d \mathbf{v}_{2}$. Clearly $f_{1,2}$ has the reduction property

$$
\int f_{1,2}^{(k, m)} d \Gamma_{2}=f_{1}^{(k)}
$$

(Here a subscript notation for evaluation at a point has been used. When a subscript is omitted, evaluation at the point $\mathbf{x}_{1}$ is implied.)

\section{EQUATION FOR THE PRESSURE}

In this section an equation for the pressure, valid at any point in the twofluid mixture, is derived. The derivation follows the usual procedure for a single, 
incompressible fluid using results from potential theory. The derivation starts by considering "ordinary" points.

An ordinary point in a fluid is defined as a point in whose neighborhood the fluid properties vary continuously. In the problem presently being considered, an ordinary point has a constant density and constant molecular viscosity corresponding to pure fluid of either of the two types 1 or 2 . By definition, any control volume containing only ordinary points will not contain a material interface. Thus at any ordinary point one can write the mass conservation equation (Aris 1962)

$$
\frac{\partial u_{j}}{\partial x_{j}}=0
$$

and the momentum conservation equation

$$
\frac{\partial u_{i}}{\partial t}+u_{j} \frac{\partial u_{i}}{\partial x_{j}}=-\frac{1}{\rho} \frac{\partial p}{\partial x_{i}}+\frac{1}{\rho} \frac{\partial \tau_{i j}}{\partial x_{j}}+g_{i}
$$

where $\rho$ is the density at the point, $p(x, t)$ is the fluid pressure, $\vec{\tau}$ is the viscous stress tensor at the point and $\mathbf{g}$ is the acceleration due to gravity.

The next step is of importance to the theory that follows in Chapter III. The goal is to eliminate the pressure from Eq. (2.13) using the incompressibility condition, Eq. (2.12). For a single incompressible fluid in an infinite space the task is easy. In that case one can take the divergence of Eq. (2.13) and use Eq. (2.12) to obtain a Poisson equation for the pressure

$$
\nabla^{2} p=-\rho \nabla \cdot \mathbf{a}
$$

where $\mathrm{a}=(\mathbf{u} \cdot \nabla) \mathbf{u}$. The solution to Eq. (2.14) in an infinite space is (Oates 1974)

$$
p\left(\mathbf{x}_{1}, t\right)=\frac{\rho}{4 \pi} \int \frac{1}{r_{12}} \nabla_{2} \cdot \mathbf{a}_{2} d \mathbf{x}_{2}
$$

where $r_{12}=\left|x_{1}-x_{2}\right|$ and the volume integral extends over all space. The pressure can be thought of as a potential at a point arising from sources distributed throughout space. The source at any point is the gradient in the vector $\mathbf{a}$, where $\mathbf{a}$ is the acceleration at a point that occurs as a result of momentum advection.

In the two-fluid case, careful account must be taken of the fact that a single fluid does not occupy the whole space. Consider a subvolume of the whole space $V^{(1)}$, occupied only by fluid of constant density $\rho^{(1)}$, and bounded by a continuous surface $S^{(1)}$. The volume $V^{(1)}$ could be a single bubble of fluid 1 , of any shape, in a sea of fluid 2. The potential $p$ at any point, inside or outside $V^{(1)}$, is (Oates 1974)

$$
\begin{aligned}
p\left(\mathbf{x}_{1}, t\right)= & \frac{\rho^{(1)}}{4 \pi} \int_{V^{(1)}} \frac{1}{r_{12}} \nabla_{2} \cdot \mathbf{a}_{2} d \mathbf{x}_{2} \\
& +\frac{1}{4 \pi} \int_{S^{(1)}}\left(\frac{1}{r_{12}} \nabla_{2} p_{2}-p_{2} \nabla_{2} \frac{1}{r_{12}}\right) \cdot \hat{\mathbb{M}}^{(1)} d s
\end{aligned}
$$


where the volume integral extends over only $V^{(1)}$ and the surface integral extends over its surface $S^{(1)}$ with outward normal $\hat{n}^{(1)}$. In this form the volume integral accounts for the contribution to the potential due to sources within $V^{(1)}$, and the surface integral accounts for all sources lying external to $V^{(1)}$. A similar expression can be written for the pressure at the same point, by considering only the volume of space not occupied by fluid $1, V^{(2)}$. The result is the same as Eq. (2.16) with superscript 1 replaced by 2 . Now observe that the surface integral in (2.16) can be replaced with the volume integral over $V^{(2)}$ with no approximation. This is because the integral over $V^{(2)}$ is exactly given by the integral over $S^{(1)}$, and the integral over $V^{(1)}$ is given exactly by the integral over $r^{(2)}$. Thus

$$
\begin{aligned}
p\left(\mathbf{x}_{1}, t\right)= & \frac{\rho^{(1)}}{4 \pi} \int_{V^{(1)}} \frac{1}{r_{12}} \nabla_{2} \cdot \mathbf{a}_{2} d \mathbf{x}_{2} \\
& +\frac{\rho^{(2)}}{4 \pi} \int_{V^{(2)}} \frac{1}{r_{12}} \nabla_{2} \cdot \mathbf{a}_{2} d \mathbf{x}_{2} .
\end{aligned}
$$

The next step is to realize that this process of subdivision and addition can be continued as much as is necessary to divide the entire flow field into simply-connected volumes all with continuous surfaces, no matter how complex the system of interfaces may be. All that is necessary is that each subvolume must contain only one material so that no discontinuity exists in any of the subvolumes. Furthermore, all of the subvolumes containing fluid 1 can be grouped into a single volume integral over the whole space by use of the material function $\theta^{(1)}$. Similarly all subvolume integrals over material 2 can be grouped into a single integral. Replacing a with $(\mathbf{u} \cdot \nabla) \mathbf{u}$, the result is

$$
p\left(\mathrm{x}_{1}, t\right)=\frac{1}{4 \pi} \sum_{k} \rho^{(k)} \int \frac{\theta_{2}^{(k)}}{r_{12}} \frac{\partial^{2}}{\partial x_{2 j} \partial x_{2 k}} u_{2 j} u_{2 k} d \mathrm{x}_{2},
$$

which is to be used to eliminate the pressure from the instantaneous equation of motion. With this and the multimaterial probability distribution functions defined in Sec. 1 , the ensemble average equations can be developed. 


\section{ENSEMBLE AVERAGE EQUATIONS}

In this section the conservation equations are derived for mean mass, momentum and fluctuational kinetic energy. The technique is a straightforward application of the definitions given in Sec. 1 of this chapter.

To derive the mean mass conservation equation, Eqs. (2.2) and (2.5) are required. These are the "conservation" law for the material function

$$
\frac{\partial \theta^{(k)}}{\partial t}+u_{j} \frac{\partial \theta^{(k)}}{\partial x_{j}}=0,
$$

and the definition of the expected material function

$$
\int \alpha f^{(k)} d \mathbf{T}=\left\langle\theta^{(k)}\right\rangle
$$

Hence

$$
\begin{aligned}
\frac{\partial}{\partial t}\left\langle\theta^{(k)}\right\rangle & =\left\langle\frac{\partial \theta^{(k)}}{\partial t}\right\rangle \\
& =-\left\langle u_{j} \frac{\partial \theta^{(k)}}{\partial x_{j}}\right\rangle \\
& =-\left\langle u_{j} \frac{\partial \theta^{(k)}}{\partial x_{j}} \int \delta\left(\theta^{(k)}-\alpha\right) \delta(\mathbf{u}-\mathbf{v}) d \Gamma\right\rangle \\
& =-\frac{\partial}{\partial x_{j}}\left\langle\int \alpha v_{j} \delta\left(\theta^{(k)}-\alpha\right) \delta(\mathbf{u}-\mathbf{v} d \Gamma\rangle\right. \\
& =-\frac{\partial}{\partial x_{j}} \int \alpha v_{j} f^{(k)} d \Gamma \\
& =-\frac{\partial}{\partial x_{j}}\left\langle u_{j} \theta^{(k)}\right\rangle .
\end{aligned}
$$

In this development, use has been made of the facts that the limits of the \langle\rangle integrations are not functions of time or space, and $\int \delta\left(\theta^{(k)}-\alpha\right) \delta(\mathbf{u}-\mathbf{v}) d \mathbf{\Gamma}=$ 1. Recalling the definition of mean velocity $\left\langle\mathbf{u}^{(k)}\right\rangle\left\langle\theta^{(k)}\right\rangle=\left\langle\theta^{(k)} \mathbf{u}\right\rangle$, the mass conservation equation is written

$$
\rho^{(k)}\left[\frac{\partial}{\partial t}\left\langle\theta^{(k)}\right\rangle+\frac{\partial}{\partial x_{j}}\left\langle u_{j}^{(k)}\right\rangle\left\langle\theta^{(k)}\right\rangle\right]=0
$$

Equation (2.19a) contains what may be called a multimaterial incompressibility condition. Dividing (2.19) through by $\rho^{(k)}$ and adding the equation for $k=1$ to 
that for $k=2$ yields

$$
\begin{aligned}
\frac{\partial}{\partial x_{i}}\left[\left\langle u_{j}^{(1)}\right\rangle\left\langle\theta^{(1)}\right\rangle+\left\langle u_{j}^{(2)}\right\rangle\left\langle\theta^{(2)}\right\rangle\right] & =\frac{\partial}{\partial x_{j}}\left[\left\langle u_{j} \theta^{(1)}\right\rangle+\left\langle u_{j} \theta^{(2)}\right\rangle\right] \\
& =\frac{\partial\left\langle u_{j}\right\rangle}{\partial x_{j}} \\
& =0 .
\end{aligned}
$$

Hence by virtue of the microscopic incompressibility of the two pure materials, the composite velocity $\langle\mathbf{u}\rangle$ is divergence free.

The mean mornentum equation cornes from the definition of the mean momentum density per unit mass

$$
\int \alpha v_{j} f^{(k)} d \mathbf{\Gamma}=\left\langle u_{j} \theta^{(k)}\right\rangle
$$

and the instantaneous equation of motion at an ordinary point

$$
\frac{\partial u_{i}}{\partial t}+u_{j} \frac{\partial u_{i}}{\partial x_{j}}=-\frac{1}{\rho} \frac{\partial p}{\partial r_{i}}+\frac{1}{\rho} \frac{\partial \tau_{i j}}{\partial x_{j}}+g_{i}
$$

and Eq. (2.2). Thus

$$
\begin{aligned}
\frac{\partial}{\partial t}\left\langle u_{i} \theta^{(k)}\right\rangle= & \left\langle u_{i} \frac{\partial \theta^{(k)}}{\partial t}+\theta^{(k)} \frac{\partial u_{i}}{\partial t}\right\rangle \\
= & -\left\langle u_{i} u_{j} \frac{\partial \theta^{(k)}}{\partial x_{j}}\right\rangle-\left\langle\theta^{(k)} u_{j} \frac{\partial u_{i}}{\partial x_{j}}\right\rangle \\
& -\left\langle\theta^{(k)} \frac{1}{\rho} \frac{\partial p}{\partial x_{i}}\right\rangle+\left\langle\theta^{(k)} \frac{1}{\rho} \frac{\partial \tau_{i j}}{\partial x_{j}}\right\rangle+\left\langle\theta^{(k)} g_{i}\right\rangle,
\end{aligned}
$$

where the first two terms on the right combine

$$
\begin{aligned}
-\left\langle u_{i} u_{j} \frac{\partial \theta^{(k)}}{\partial x_{j}}+\theta^{(k)} u_{j} \frac{\partial u_{i}}{\partial x_{j}}\right\rangle & =-\left\langle u_{j} \frac{\partial}{\partial x_{j}} u_{i} \theta^{(k)}\right\rangle \\
& =-\left\langle\frac{\partial}{\partial x_{j}} u_{j} u_{i} \theta^{(k)}\right\rangle \\
& =-\frac{\partial}{\partial x_{j}}\left\langle u_{j} u_{i} \theta^{(k)}\right\rangle
\end{aligned}
$$

in which the condition $\nabla \cdot \mathbf{u}=\mathbf{0}$ has been used. So far the momentum equation is 


$$
\begin{aligned}
\frac{\partial}{\partial t}\left\langle u_{i} \theta^{(k)}\right\rangle+\frac{\partial}{\partial x_{j}}\left\langle u_{j} u_{i} \theta^{(k)}\right\rangle= & -\frac{1}{\rho^{(k)}}\left\langle\theta^{(k)} \frac{\partial p}{\partial x_{i}}\right\rangle \\
& +\frac{1}{\rho^{(k)}}\left\langle\theta^{(k)} \frac{\partial \tau_{i j}}{\partial x_{j}}\right\rangle \\
& +g_{i}\left\langle\theta^{(k)}\right\rangle .
\end{aligned}
$$

In obtaining Eq. (2.20), use has been made of the fact that $\rho=\rho^{(k)}$ for those members of the ensemble in which $\theta^{(k)}=1$, which are the only members of the ensemble that contribute to the expectation.

To place Eq. (2.20) in a more convenient form, let

$$
u_{i}=\left\langle u_{i}^{(k)}\right\rangle+C_{i}^{(k)}
$$

and

$$
p=\langle p\rangle+p^{\prime}
$$

where $p^{\prime}=p-\langle p\rangle$ and again $C_{i}^{(k)}=u_{i}-\left\langle u_{1}^{(k)}\right\rangle$. Note that $\left\langle p^{\prime}\right\rangle=0$ but that in general $\left\langle C_{1}^{(k)}\right\rangle \neq 0$. However,

$$
\begin{aligned}
\left\langle C_{i}^{(k)} \theta^{(k)}\right\rangle & =\left\langle\left(u_{i}-\left\langle u_{i}^{(k)}\right\rangle\right) \theta^{(k)}\right\rangle \\
& =\left\langle u_{i} \theta^{(k)}\right\rangle-\left\langle u_{i}^{(k)}\right\rangle\left\langle\theta^{(k)}\right\rangle \\
& =0
\end{aligned}
$$

by definition of the mean velocity. With these the advection term of Eq. (2.20) becomes

$$
\begin{aligned}
\left\langle u_{j} u_{i} \theta^{(k)}\right\rangle & =\left\langle\left(\left\langle u_{j}^{(k)}\right\rangle+C_{j}^{(k)}\right)\left(\left\langle u_{i}^{(k)}\right\rangle+C_{i}^{(k)}\right) \theta^{(k)}\right\rangle \\
& =\left\langle u_{j}^{(k)}\right\rangle\left\langle u_{i}^{(k)}\right\rangle\left\langle\theta^{(k)}\right\rangle+\left\langle C_{j}^{(k)} C_{i}^{(k)} \theta^{(k)}\right\rangle \\
& =\left\langle u_{j}^{(k)}\right\rangle\left\langle u_{i} \theta^{(k)}\right\rangle+\left\langle C_{j}^{(k)} C_{i}^{(k)} \theta^{(k)}\right\rangle,
\end{aligned}
$$

(2.22) and the pressure term becomes

$$
\begin{aligned}
-\frac{1}{\rho^{(k)}}\left\langle\theta^{(k)} \frac{\partial p}{\partial x_{i}}\right\rangle & =-\frac{1}{\rho^{(k)}}\left\langle\theta^{(k)}\right\rangle \frac{\partial\langle p\rangle}{\partial x_{i}}-\frac{1}{\rho^{(k)}}\left\langle\theta^{(k)} \frac{\partial p^{\prime}}{\partial x_{i}}\right\rangle \\
& =-\frac{1}{\rho^{(k)}}\left\langle\theta^{(k)}\right\rangle \frac{\partial\langle p\rangle}{\partial x_{i}}-\frac{1}{\rho^{(k)}} \frac{\partial}{\partial x_{i}}\left\langle\theta^{(k)} p^{\prime}\right\rangle+\frac{1}{\rho^{(k)}}\left\langle p^{\prime} \frac{\partial \theta^{(k)}}{\partial x_{i}}\right\rangle
\end{aligned}
$$


Note that the last part is an interface term, by virtue of the gradient in the discontinuous function $\theta^{(k)}$.

The viscous stress at an "ordinary" point in an incompressible fluid is

$$
\tau_{i j}=\mu\left(\frac{\partial u_{i}}{\partial x_{j}}+\frac{\partial u_{j}}{\partial x_{i}}\right)
$$

where $\mu$ is the molecular viscosity of the fluid. The expected stress in material $k$ at a point is found by

$$
\begin{aligned}
\left\langle\theta^{(k)} \tau_{i j}\right\rangle= & \left\langle\theta^{(k)} \mu\left(\frac{\partial u_{i}}{\partial x_{j}}+\frac{\partial u_{j}}{\partial x_{i}}\right)\right\rangle \\
= & \mu^{(k)}\left\langle\theta^{(k)}\right\rangle\left(\frac{\partial}{\partial x_{j}}\left\langle u_{i}^{(k)}\right\rangle+\frac{\partial}{\partial x_{i}}\left\langle u_{j}^{(k)}\right\rangle\right) \\
& +\mu^{(k)}\left\langle\theta^{(k)}\left(\frac{\partial}{\partial x_{j}} C_{i}^{(k)}+\frac{\partial}{\partial x_{i}} C_{j}^{(k)}\right)\right\rangle,
\end{aligned}
$$

where the fact that $\mu=\mu^{(k)}$ in the nonzero members of the ensemble has been used. Now notice that the second part is zero. This can be seen by inserting $\int \delta\left(\theta^{(l)}-\alpha\right) \delta(\mathbf{u}-\mathbf{v}) d \boldsymbol{\Gamma}$ under the gradient operator. For example,

$$
\begin{aligned}
\left\langle\theta^{(k)} \frac{\partial}{\partial x_{j}} \int\left(v_{i}-\left\langle u_{i}^{(k)}\right\rangle\right)\right. & \left.\delta\left(\theta^{(k)}-\alpha\right) \delta(\mathbf{u}-\mathbf{v}) d \mathbf{\Gamma}\right\rangle \\
& =\int \alpha \frac{\partial}{\partial x_{j}}\left(v_{i}-\left\langle u_{i}^{(k)}\right\rangle\right) f^{(k)} d \mathbf{\Gamma} \\
& =\frac{\partial}{\partial x_{j}} \int \alpha\left(v_{i}-\left\langle u_{i}^{(k)}\right\rangle\right) f^{(k)} d \mathbf{\Gamma} \\
& =0,
\end{aligned}
$$

Thus the average stress in material $k$ is

$$
\left\langle\theta^{(k)} \tau_{i j}\right\rangle=\mu^{(k)}\left\langle\theta^{(k)}\right\rangle D_{i j}^{(k)}
$$

where the rate of strain tensor $D_{i j}^{(k)}$ is defined

$$
D_{i j}^{(k)}=\frac{\partial}{\partial x_{j}}\left\langle u_{i}^{(k)}\right\rangle+\frac{\partial}{\partial x_{i}}\left\langle u_{j}^{(k)}\right\rangle
$$

This is used to rewrite the viscous term in Eq. (2.20) 


$$
\begin{aligned}
\frac{1}{\rho^{(k)}}\left\langle\theta^{(k)} \frac{\partial}{\partial x_{j}} \tau_{i j}\right\rangle= & \frac{1}{\rho^{(k)}} \frac{\partial}{\partial x_{j}} \mu^{(k)}\left\langle\theta^{(k)}\right\rangle D_{i j}^{(k)} \\
& -\frac{1}{\rho^{(k)}}\left\langle\tau_{i j} \frac{\partial \theta^{(k)}}{\partial x_{j}}\right\rangle
\end{aligned}
$$

where the second part is seen to contain the gradient in the material function $\theta^{(k)}$. As noted earlier $\nabla \theta^{(k)}=0$ except at a material interface where it is infinite. This can be handled mathematically by imagining an interfacial region of finite width over which the material function varies smoothly from zero to one. In the limit of zero width for the interface region, $\nabla \theta^{(k)}$ becomes a delta function times a unit normal at the interface, directed away from material $k$. This representation will be utilized later. For now, the foregoing terms are inserted into Eq. (2.20) to yield the mean momentum equation

$$
\begin{aligned}
& \rho^{(k)}\left[\frac{\partial}{\partial t}\left\langle u_{i} \theta^{(k)}\right\rangle+\frac{\partial}{\partial x_{j}}\left\langle u_{j}^{(k)}\right\rangle\left\langle u_{i} \theta^{(k)}\right\rangle\right] \\
&=-\left\langle\theta^{(k)}\right\rangle \frac{\partial}{\partial x_{i}}\langle p\rangle \\
&+\frac{\partial}{\partial x_{j}} \mu^{(k)}\left\langle\theta^{(k)}\right\rangle D_{i j}^{(k)} \\
&+\rho^{(k)}\left\langle\theta^{(k)}\right\rangle g_{i} \\
&-\rho^{(k)} \frac{\partial}{\partial x_{j}}\left\langle C_{i}^{(k)} C_{j}^{(k)} \theta^{(k)}\right\rangle \\
&+\left\langle\left(p^{\prime} \delta_{i j}-\tau_{i j}\right) \frac{\partial \theta^{(k)}}{\partial x_{j}}\right\rangle \\
&-\frac{\partial}{\partial x_{i}}\left\langle\theta^{(k)} p^{\prime}\right\rangle,
\end{aligned}
$$

where the mean rate of strain tensor $D_{i j}^{(k)}$ is given by Eq. (2.21).

The equation for fluctuational kinetic energy in material $k$ comes from the definition of Eq. (2.9)

$$
\frac{1}{2} \int \alpha\left(v_{i}-\left\langle u_{i}^{(k)}\right\rangle\right)^{2} f^{(k)} d \mathbf{\Gamma}=\left\langle\frac{1}{2} C_{i}^{(k) 2} \theta^{(k)}\right\rangle
$$

together with Eqs. (2.2) and (2.13). Hence 


$$
\begin{aligned}
\frac{\partial}{\partial t}\left\langle\frac{1}{2} C_{i}^{(k) 2} \theta^{(k)}\right\rangle= & \left\langle\frac{1}{2} \frac{\partial}{\partial t} C_{i}^{(k) 2} \theta^{(k)}\right\rangle \\
= & \left\langle C_{i}^{(k)} \theta^{(k)} \frac{\partial C_{i}^{(k)}}{\partial t}\right\rangle+\left\langle\frac{1}{2} C_{i}^{(k) 2} \frac{\partial \theta^{(k)}}{\partial t}\right\rangle \\
= & \left\langle C_{i}^{(k)} \theta^{(k)} \frac{\partial u_{i}}{\partial t}\right\rangle-\left\langle C_{i}^{(k)} \theta^{(k)}\right\rangle \frac{\partial}{\partial t}\left\langle u_{i}^{(k)}\right\rangle+\left\langle\frac{1}{2} C_{i}^{(k) 2} \frac{\partial \theta^{(k)}}{\partial t}\right\rangle \\
= & -\left\langle C_{i}^{(k)} \theta^{(k)} u_{j} \frac{\partial u_{i}}{\partial x_{j}}\right\rangle-\left\langle C_{i}^{(k)} \theta^{(k)} \frac{1}{\rho} \frac{\partial p}{\partial x_{i}}\right\rangle+\left\langle C_{i}^{(k)} \theta^{(k)} \frac{1}{\rho} \frac{\partial \tau_{i j}}{\partial x_{j}}\right\rangle \\
& +\left\langle C_{i}^{(k)} \theta^{(k)} g_{i}\right\rangle-\left\langle\frac{1}{2} C_{i}^{(k) 2} \theta^{(k)} u_{j} \frac{\partial \theta^{(k)}}{\partial x_{j}}\right\rangle
\end{aligned}
$$

The first and last terms on the right of Eq. (2.23) can be combined. Thus

$$
\begin{aligned}
-\left\langle C_{i}^{(k)} \theta^{(k)} u_{j} \frac{\partial u_{i}}{\partial x_{j}}\right\rangle & -\left\langle\frac{1}{2} C_{i}^{(k) 2} \theta^{(k)} u_{j} \frac{\partial \theta^{(k)}}{\partial x_{j}}\right\rangle=-\frac{\partial}{\partial x_{j}}\left\langle u_{j}^{(k)}\right\rangle\left\langle\frac{1}{2} C_{j}^{(k) 2} \theta^{(k)}\right\rangle \\
& -\frac{\partial}{\partial x_{j}}\left\langle\frac{1}{2} C_{j}^{(k)} C_{i}^{(k) 2} \theta^{(k)}\right\rangle-\left\langle C_{i}^{(k)} C_{j}^{(k)} \theta^{(k)}\right\rangle \frac{\partial}{\partial x_{j}}\left\langle u_{i}^{(k)}\right\rangle
\end{aligned}
$$

The pressure term in Eq. (2.23) can be written

$$
-\left\langle C_{i}^{(k)} \theta^{(k)} \frac{1}{\rho} \frac{\partial p}{\partial x_{i}}\right\rangle=-\frac{1}{\rho^{(k)}}\left\langle C_{i}^{(k)} \theta^{(k)} \frac{\partial p^{\prime}}{\partial x_{i}}\right\rangle
$$

because $\rho=\rho^{(k)}$ for the nonzero points in the expectation and $\left\langle C_{i}^{(k)} \theta^{(k)}\right\rangle=0$. This pressure term can be expressed more conveniently by writing the expectation as a sum of three parts

$$
\begin{aligned}
-\left\langle C_{i}^{(k)} \theta^{(k)} \frac{\partial p^{\prime}}{\partial x_{i}}\right\rangle= & -\frac{\partial}{\partial x_{i}}\left\langle C_{i}^{(k)} \theta^{(k)} p^{\prime}\right\rangle \\
& +\left\langle C_{i}^{(k)} p^{\prime} \frac{\partial \theta^{(k)}}{\partial x_{i}}\right\rangle+\left\langle\theta^{(k)} p^{\prime} \frac{\partial C_{i}^{(k)}}{\partial x_{i}}\right\rangle
\end{aligned}
$$

in which

$$
\frac{\partial C_{i}^{(k)}}{\partial x_{i}}=\frac{\partial}{\partial x_{i}}\left(u_{i}-\left\langle u_{i}^{(k)}\right\rangle\right)=-\frac{\partial}{\partial x_{i}}\left\langle u_{i}^{(k)}\right\rangle,
$$

since $\nabla \cdot \mathbf{u}=\mathbf{0}$. Because the composite velocity $\langle\mathbf{u}\rangle$ is divergence free, this can be written 


$$
\frac{\partial C_{i}^{(k)}}{\partial x_{i}}=-\frac{\partial}{\partial x_{i}}\left\langle u_{i}^{(k)}\right\rangle=\frac{\partial}{\partial x_{i}}\left[\left\langle u_{i}\right\rangle-\left\langle u_{i}^{(k)}\right\rangle\right]=\frac{\partial}{\partial x_{i}}\left\langle C_{i}^{(k)}\right\rangle .
$$

Thus the last part can be rewritten

$$
\begin{aligned}
\left\langle\theta^{(k)} p^{\prime} \frac{\partial C_{i}^{(k)}}{\partial x_{i}}\right\rangle & =\left\langle\theta^{(k)} p^{\prime}\right\rangle \frac{\partial}{\partial x_{i}}\left\langle C_{i}^{(k)}\right\rangle \\
& =\frac{\partial}{\partial x_{i}}\left\langle C_{i}^{(k)}\right\rangle\left\langle\theta^{(k)} p^{\prime}\right\rangle-\left\langle C_{i}^{(k)}\right\rangle \frac{\partial}{\partial x_{i}}\left\langle\theta^{(k)} p^{\prime}\right\rangle
\end{aligned}
$$

The viscous term in Eq. (2.24) is written by analogy to the viscous term in the momentum equation. Thus

$$
\begin{aligned}
\left\langle C_{i}^{(k)} \theta^{(k)} \frac{1}{\rho} \frac{\partial \tau_{i j}}{\partial x_{j}}\right\rangle= & \frac{1}{\rho^{(k)}} \frac{\partial}{\partial x_{j}}\left\langle\theta^{(k)} C_{i}^{(k)} \tau_{i j}\right\rangle \\
& -\frac{1}{\rho^{(k)}}\left\langle\theta^{(k)} \tau_{i j} \frac{\partial}{\partial x_{j}} C_{i}^{(k)}\right\rangle \\
& -\frac{1}{\rho^{(k)}}\left\langle\tau_{i j} C_{i}^{(k)} \frac{\partial \theta^{(k)}}{\partial x_{j}}\right\rangle
\end{aligned}
$$

where the first term on the right becomes

$$
\frac{1}{\rho^{(k)}} \frac{\partial}{\partial x_{j}}\left\langle\theta^{(k)} C_{i}^{(k)} \tau_{i j}\right\rangle=\frac{1}{\rho^{(k)}} \frac{\partial}{\partial x_{j}} \mu^{(k)}\left\langle\theta^{(k)} C_{i}^{(k)}\left(\frac{\partial C_{i}^{(k)}}{\partial x_{j}}+\frac{\partial C_{j}^{(k)}}{\partial x_{i}}\right)\right\rangle
$$

the second term on the right becomes

$$
-\frac{1}{\rho^{(k)}}\left\langle\theta^{(k)} \tau_{i j} \frac{\partial C_{i}^{(k)}}{\partial x_{j}}\right\rangle=-\frac{\mu^{(k)}}{\rho^{(k)}}\left\langle\theta^{(k)}\left(\frac{\partial C_{i}^{(k)}}{\partial x_{j}}+\frac{\partial C_{j}^{(k)}}{\partial x_{i}}\right) \frac{\partial C_{i}^{(k)}}{\partial x_{j}}\right\rangle
$$

and the third term is another interface term. Since the acceleration due to gravity is a constant

$$
\left\langle C_{i}^{(k)} \theta^{(k)} g_{i}\right\rangle=g_{i}\left\langle C_{i}^{(k)} \theta^{(k)}\right\rangle=0 .
$$

Thus the equation for fluctuarional kinetic energy is 


$$
\begin{aligned}
\rho^{(k)}\left[\frac{\partial}{\partial t}\left\langle\frac{1}{2} C_{i}^{(k) 2} \theta^{(k)}\right\rangle\right. & \left.+\frac{\partial}{\partial x_{j}}\left\langle u_{j}^{(k)}\right\rangle\left\langle\frac{1}{2} C_{i}^{(k) 2} \theta^{(k)}\right\rangle\right] \\
= & -\rho^{(k)}\left\langle C_{i}^{(k)} C_{j}^{(k)} \theta^{(k)}\right\rangle \frac{\partial}{\partial x_{j}}\left\langle u_{i}^{(k)}\right\rangle \\
& -\frac{\partial}{\partial x_{j}} \rho^{(k)}\left\langle\frac{1}{2} C_{j}^{(k)} C_{i}^{(k) 2} \theta^{(k)}\right\rangle \\
& +\frac{\partial}{\partial x_{j}} \mu^{(k)}\left\langle C_{i}^{(k)} \theta^{(k)}\left(\frac{\partial C_{i}^{(k)}}{\partial x_{j}}+\frac{\partial C_{j}^{(k)}}{\partial x_{i}}\right)\right\rangle \\
& -\mu^{(k)}\left\langle\theta^{(k)}\left(\frac{\partial C_{i}^{(k)}}{\partial x_{j}}+\frac{\partial C_{j}^{(k)}}{\partial x_{i}}\right) \frac{\partial C_{i}^{(k)}}{\partial x_{j}}\right\rangle \\
& +\left\langle\left(p^{\prime} \delta_{i j}-\tau_{i j}\right) C_{i}^{(k)} \frac{\partial \theta^{(k)}}{\partial x_{j}}\right\rangle \\
& -\frac{\partial}{\partial x_{i}}\left\langle C_{i}^{(k)} \theta^{(k)} p^{\prime}\right\rangle \\
& -\left\langle C_{i}^{(k)}\right\rangle \frac{\partial}{\partial x_{i}}\left\langle\theta^{(k)} p^{\prime}\right\rangle \\
& +\frac{\partial}{\partial x_{i}}\left\langle C_{i}^{(k)}\right\rangle\left\langle\theta^{(k)} p^{\prime}\right\rangle
\end{aligned}
$$

\section{SUMMARY}

This section is a summary and discussion of the average equations for mass, momentum, and fuctuational kinetic energy that have just been developed. Even though these equations are as yet an unclosed set, they contain a variety of interesting features. For the convenience of discussion, the unclosed average equations are collected together here. The mass conservation cquation is

$$
\rho^{(k)}\left[\frac{\partial}{\partial t}\left\langle\theta^{(k)}\right\rangle+\frac{\partial}{\partial x_{j}}\left\langle u_{j}^{(k)}\right\rangle\left\langle\theta^{(k)}\right\rangle\right]=0,
$$

the momentum conservation equation is

$$
\rho^{(k)}\left[\frac{\partial}{\partial t}\left\langle u_{i} \theta^{(k)}\right\rangle+\frac{\partial}{\partial x_{j}}\left\langle u_{j}^{(k)}\right\rangle\left\langle u_{i} \theta^{(k)}\right\rangle\right]=-\left\langle\theta^{(k)}\right\rangle \frac{\partial}{\partial x_{i}}\langle p\rangle+\rho^{(k)}\left\langle\theta^{(k)}\right\rangle g_{i}(2.22)(a)
$$




$$
\begin{aligned}
& +\frac{\partial}{\partial x_{j}}\left[\mu^{(k)}\left\langle\theta^{(k)}\right\rangle D_{i j}^{(k)}-\rho^{(k)}\left\langle C_{i}^{(k)} C_{j}^{(k)} \theta^{(k)}\right\rangle\right] \\
& +\left\langle\left(p^{\prime} \delta_{i j}-\tau_{i j}\right) \frac{\partial \theta^{(k)}}{\partial x_{j}}\right\rangle \\
& -\frac{\partial}{\partial x_{i}}\left\langle\theta^{(k)} p^{\prime}\right\rangle
\end{aligned}
$$

and the conservation equation for fluctuational kinetic energy is

$$
\begin{aligned}
\rho^{(k)}\left[\frac{\partial}{\partial t}\left\langle\frac{1}{2} C_{i}^{(k) 2} \theta^{(k)}\right\rangle\right. & \left.+\frac{\partial}{\partial x_{j}}\left\langle u_{j}^{(k)}\right\rangle\left\langle\frac{1}{2} C_{i}^{(k) 2} \theta^{(k)}\right\rangle\right] \\
= & -\rho^{(k)}\left\langle C_{i}^{(k)} C_{j}^{(k)} \theta^{(k)}\right\rangle \frac{\partial}{\partial x_{j}}\left\langle u_{i}^{(k)}\right\rangle \\
& -\frac{\partial}{\partial x_{j}}\left[\rho^{(k)}\left\langle\frac{1}{2} C_{j}^{(k)} C_{i}^{(k) 2} \theta^{(k)}\right\rangle+\left\langle C_{j}^{(k)} \theta^{(k)} p^{\prime}\right\rangle\right] \\
& +\frac{\partial}{\partial x_{j}} \mu^{(k)}\left\langle C_{i}^{(k)} \theta^{(k)}\left(\frac{\partial C_{i}^{(k)}}{\partial x_{j}}+\frac{\partial C_{j}^{(k)}}{\partial x_{i}}\right)\right\rangle \\
& -\mu^{(k)}\left\langle\theta^{(k)}\left(\frac{\partial C_{i}^{(k)}}{\partial x_{j}}+\frac{\partial C_{j}^{(k)}}{\partial x_{i}}\right) \frac{\partial C_{i}^{(k)}}{\partial x_{j}}\right\rangle \\
& +\left\langle\left(p^{\prime} \delta_{i j}-\tau_{i j}\right) C_{i}^{(k)} \frac{\partial \theta^{(k)}}{\partial x_{j}}\right\rangle \\
& -\left\langle C_{i}^{(k)}\right\rangle \frac{\partial}{\partial x_{i}}\left\langle\theta^{(k)} p^{\prime}\right\rangle \\
& +\frac{\partial}{\partial x_{i}}\left\langle C_{i}^{(k)}\right\rangle\left\langle\theta^{(k)} p^{\prime}\right\rangle
\end{aligned}
$$

The first thing to observe about Eqs. (2.19), (2.22), and (2.24) is that in the case of only one fluid, these reduce to the ensemble average equations for a single, incompressible, turbulent fluid. For the single-fluid case the superscript can be ignored and $\theta=\langle\theta\rangle=1$ everywhere. Thus lines (c) and (d) of Eq. (2.22), and lines (e), (f), and (g) of Eq. (2.24) vanish for a single fluid. Equation (2.19) becomes $\nabla \cdot \mathbf{u}=0$. Line (b) of Eq. (2.22) contains the well-known Reynolds stress term. In Eq. (2.24), line (a) is the so-called turbulence creation term, line (b) is the energy diffusion term, line (c) is the viscous work term, and line (d) is the energy dissipation term, all in thie single-fluid case. 
By analogy to single-fluid turbulence theory, the quantity $-\left\langle C_{i}^{(k)} C_{j}^{(k)} \theta^{(k)}\right\rangle$ will be called the Reynolds stress per unit mass in material $k$, and denoted by $R_{i j}^{(k)}$. This can be written in terms of the distribution function $f^{(k)}$. Thus

$$
\begin{aligned}
R_{i j}^{(k)} & =-\left\langle C_{i}^{(k)} C_{j}^{(k)} \theta^{(k)}\right\rangle \\
& =-\left\langle C_{i}^{(k)} C_{j}^{(k)} \theta^{(k)} \int \delta\left(\theta^{(k)}-\alpha\right) \delta(\mathbf{u}-\mathbf{v}) d \mathbf{\Gamma}\right\rangle \\
& =-\int\left(v_{i}-\left\langle u_{i}^{(k)}\right\rangle\right)\left(v_{j}-\left\langle u_{j}^{(k)}\right\rangle\right) \alpha f^{(k)} d \mathbf{\Gamma}
\end{aligned}
$$

The quantity $-\left\langle\frac{1}{2} C_{j}^{(k)} C_{i}^{(k) 2} \theta^{(k)}\right\rangle$ will be called the energy flux vector for material $k$. It can be written

$$
\begin{aligned}
Q_{j}^{(k)} & =-\left\langle\frac{1}{2} C_{j}^{(k)} C_{i}^{(k) 2} \theta^{(k)}\right\rangle \\
& =-\frac{1}{2} \int\left(v_{j}-\left\langle u_{j}^{(k)}\right\rangle\right)\left(v_{i}-\left\langle u_{i}^{(k)}\right\rangle\right)^{2} \alpha f^{(k)} d \mathbf{\Gamma} .
\end{aligned}
$$

Terms (c) and (d) in Eq. (2.24) will be called the viscous work and dissipation terms respectively. These too can be expressed in terms of $f^{(k)}$, which will be shown in the next chapter. Thus the Reynolds stress, energy flux, viscous work, and dissipation are all functions of the one-point probability distribution function $f^{(k)}$. For this reason, these terms will be collectively called one-point terms.

Line (d) of Eq. (2.22) and lines (b), (f), and (g) of Eq. (2.24) will be seen to depend upon integrals over the two-point distribution function $f_{1,2}^{(k, m)}$. These terms will be called two-point terms because of their dependence on two-point statistical quantities. The two-point term in Eq. (2.22) represents a conservative exchange in momentum between the two fluids. This can be seen by using the identity $\theta^{(1)}+\theta^{(2)}=1$ so that

$$
\frac{\partial}{\partial x_{i}}\left\langle\theta^{(1)} p^{\prime}\right\rangle=-\frac{\partial}{\partial x_{i}}\left\langle\theta^{(2)} p^{\prime}\right\rangle .
$$

Thus the acceleration experienced by fluid one is the negative of the acceleration experienced by fluid two, as a result of an interaction accounted for by these terms. Therefore the two-point term in line (d) of Eq. (2.22) will also be referred to as the momentum exchange term.

Line (f) of Eq. (2.24) is a two-point term that will be seen to account for a positive-definite contribution to the fluctuational kinetic energy as a result of interpenetrating motion. This is a multimaterial turbulence creation term that is 
unique to interpenetrating flows. The quantity $\left\langle C_{i}^{(k)} \theta^{(k)} p^{\prime}\right\rangle$ is a diffusive flux of turbulence energy that results from pressure fluctuations. This appears on line (b) of Eq. (2.24) and constitutes the multimaterial analog of the pressure work term in classical turbulence theory. Like all of the terms containing the pressur. fluctuation $p^{\prime}$, it is a two-point term.

The two-point nature of the $p^{\prime}$ terms can be readily seen by using Eq. (2.18) to eliminate the pressure. First, consider the mean pressure by ensemble averaging Eq. (2.18). This is

$$
\begin{aligned}
\langle p\rangle & =\frac{1}{4 \pi}\left\langle\sum_{m} \rho^{(m)} \int \frac{\theta_{2}^{(m)}}{r_{12}} \frac{\partial^{2}}{\partial x_{2 j} \partial x_{2 k}} u_{2 j} u_{2 k} d x_{2}\right\rangle \\
& =\frac{1}{4 \pi} \sum_{m} \rho^{(m)} \int \frac{1}{r_{12}} \frac{\partial^{2}}{\partial x_{2 j} \partial x_{2 k}} \int \alpha_{2} v_{2 j} v_{2 k} f_{2}^{(m)} d \mathbf{\Gamma}_{2} d \mathbf{x}_{2}
\end{aligned}
$$

which follows directly from Eq. (2.18) by inserting $\int \delta\left(\theta_{2}^{(m)}-\alpha\right) \delta\left(\mathbf{u}_{2}-\mathbf{v}_{2}\right) d \mathbf{r}_{2}$ inside the expectation and rearranging. This can be used in the pressure part of the momentum exchange term. With $p^{\prime}=p-\langle p\rangle$, this is

$$
\begin{aligned}
& \frac{\partial}{\partial x_{i}}\left\langle\theta^{(k)}(p-\langle p\rangle)\right\rangle \\
& =\frac{\partial}{\partial x_{i}}\left\langle\theta^{(k)}\left[\frac{1}{4 \pi} \sum_{m} \rho^{(m)} \int \frac{\theta_{2}^{(m)}}{r_{12}} \frac{\partial^{2}}{\partial x_{2 j} \partial x_{2 k}} u_{2 j} u_{2 k} d \mathbf{x}_{2}-\langle p\rangle\right]\right\rangle \\
& =\frac{\partial}{\partial x_{j}}\left\langle\int \alpha_{1} \delta\left(\theta_{1}^{(k)}-\alpha_{1}\right) \delta\left(\mathbf{u}_{1}-\mathbf{v}_{1}\right) d \mathbf{\Gamma}_{1}\right. \\
& {\left[\frac{1}{4 \pi} \sum_{m} \rho^{(m)} \int \frac{1}{r_{12}} \frac{\partial^{2}}{\partial x_{2 j} \partial x_{2 k}} \int \alpha_{2} v_{2 j} v_{2 k} \delta\left(\theta_{2}^{(m)}-\alpha_{2}\right) \delta\left(\mathbf{u}_{2}-\mathbf{v}_{2}\right) d \mathbf{r}_{2} d \mathbf{x}_{2}\right.} \\
& \left.\left.-\frac{1}{4 \pi} \sum_{m} \rho^{(m)} \int \frac{1}{r_{12}} \frac{\partial^{2}}{\partial x_{2 j} \partial x_{2 k}} \int \alpha_{2} v_{2 j} v_{2 k} f_{2}^{(m)} d \mathbf{r}_{2} d \mathbf{x}_{2}\right]\right\rangle \\
& =\frac{1}{4 \pi} \int \frac{\partial}{\partial x_{1 i}} \frac{1}{r_{12}} \frac{\partial^{2}}{\partial x_{2 j} \partial x_{2 k}}\left[\sum_{m} \rho^{(m)} \int \alpha_{1} \alpha_{2} v_{2 j} v_{2 k}\left(f_{1,2}^{(k, m)}-f_{1}^{(k)} f_{2}^{(m)}\right) d \mathbf{\Gamma}_{1,2}\right] d \mathbf{x} \\
& =\frac{1}{4 \pi} \int \frac{\partial}{\partial x_{1 i}} \frac{1}{r_{12}} \frac{\partial^{2}}{\partial x_{2 j} \partial x_{2 k}} A_{j k}^{(k)} d \mathbf{x}_{2},
\end{aligned}
$$

where 


$$
A_{j k}^{(k)}=\sum_{m} \rho^{(m)} \int \alpha_{1} \alpha_{2} v_{2 j} v_{2 k}\left(f_{1,2}^{(k, m)}-f_{1}^{(k)} f_{2}^{(m)}\right) d \mathbf{\Gamma}_{1,2}
$$

The quantity $\left(f_{1,2}^{(k, m)}-f_{1}^{(k)} f_{2}^{(m)}\right)$ is a correlation function. If points one and two are statistically independent $f_{1,2}^{(k, m)}=f_{1}^{(k)} f_{2}^{(m)}$ and the correlation function is zero. Therefore the tensor $A_{i j}^{(k)}$ will be called a correlation coefficient. In the next chapter these correlation coefficients will be modeled on physical grounds in a way that enables Eq. (2.27) to be evaluated in a certain approximation. This provides a closure technique that is based on what may be called a theory of correlation coefficients.

Line (c) of the momentum equation and line (e) of the energy equation were referred to earlier as interface terms. In a sense these are also two-point terms. This is most easily demonstrated by considering the integral form of the equations. The integral form is obtained by integrating the differential equations over an arbitrary volume $V$. Consider first the viscous part of line (c), Eq. (2.22). The integral form is

$$
\begin{aligned}
-\int_{V}\left\langle\tau_{i j} \frac{\partial \theta^{(k)}}{\partial x_{j}}\right\rangle d \mathbf{x}_{2} & =-\left\langle\int_{V} \tau_{2 i j} \delta\left(\mathbf{x}_{2}-\mathbf{x}_{s}\right) \hat{n}_{2 j}^{(k)} d \mathbf{x}_{2}\right\rangle \\
& =-\left\langle\int_{S} \tau_{2 i j} \hat{n}_{2 j}^{(k)} d \mathbf{x}_{s}\right\rangle
\end{aligned}
$$

where $\mathbf{x}_{s}$ are the coordinates of interface points within the arbitrary volume $V$. Here the discontinuous nature of $\theta^{(k)}$ has been used. The gradient in $\theta^{(k)}$ is zero except at an interface where it is a delta function times a unit normal $\hat{\mathbf{n}}^{(k)}$ directed away from material $k$. Thus the volume integral becomes an integral over the interfacial area contained within $V$.

In a similar way the integral form of the viscous part of the turbulence creation term becomes

$$
-\int\left\langle\tau_{i j} C_{i}^{(k)} \frac{\partial \theta^{(k)}}{\partial x_{j}}\right\rangle d \mathbf{x}_{2}=-\left\langle\int_{S} \tau_{2 i j} C_{2 i}^{(k)} \hat{n}_{2 j}^{(k)} d \mathbf{x}_{s}\right\rangle .
$$

Equations (2.29) and (2.30) are two-point terms because the stress at an interface point is properly obtained by a limiting process in which a volume element containing the interface point is allowed to become small. Unfortunately a statistical description of the interface geometry is required in order to express terms like (2.29) and (2.30) as integrals over a distribution function (Lundgren 1972). Therefore the interface terms are handled in a more heuristic fashion that is detailed in Sec. 4 of the next chapter. 
The last important point to be made here is that Eqs. (2.19), (2.22), and (2.24) are relevant to the case of an incompressible fluid with suspended rigid solid fragments, as well as the two-fluid case. In the case of suspended rigid solids, where $k=1$ for the solid material say, the value $\mu^{(1)}=0$ in Eqs. (2.22) and (2.24) can be used to obtain the proper average equations. The proof of this is in the fact that Eq. (2.13) is an appropriate equation of motion for any point in the flow, even if the point is in a rigid body.

To see this, let $\mathbf{v}(t)$ be the velocity of the center of mass of a rigid body moving in a fluid. If the mass of the body is $m$, the statement of momentum conservation is

$$
\frac{d}{d t} m v_{i}=-\int_{S} p \hat{n}_{i} d s+\int_{S} \tau_{i j} \hat{n}_{j} d s+m g_{i}
$$

where the integrals extend over the surface of the body, $p$ is the pressure and $\tau_{i j}$ is the viscous stress at the surface with outward normal $\hat{n}$. The viscous stress $\tau_{i j}$ is not defined in the rigid body. It can be thought of as being uniformly zero on the interior of the body, with a discontinuous jump to $\tau_{i j}$ at the surface. For this purpose $\tau_{i j}$ at the surface is considered to be obtained from a limiting process by approaching the surface through points in the fluid.

Now let $\mathbf{u}(\mathbf{x}, t)$ be the velocity in the interior of the body. By definition,the total linear momentum of the body is

$$
\int_{V} \rho \mathbf{u}(\mathbf{x}, t) d V=m \mathbf{v}
$$

where $\rho$ is the constant density of the rigid material. Equation (2.32) can be placed in Eq. (2.31) and the time derivative can be brought inside the integral to yield

$$
\rho \int_{V}\left(\frac{\partial u_{i}}{\partial t}+u_{j} \frac{\partial u_{i}}{\partial x_{j}}\right) d V=-\int_{V} \frac{\partial p}{\partial x_{i}} d V+\int_{V} \frac{\partial \tau_{i j}}{\partial x_{j}}+\rho g_{i} \int_{V} d V
$$

where the Reynolds transport theorem and the divergence theorem have been used (Aris 1962). Equation (2.33) can be considered to be valid for any contrsi volume inside the rigid body with $\tau_{i j}=0$, provided that $p(\mathbf{x}, t)$ in the body is regarded as a potential given by Eq. (2.18) and provided that the effects of finite $\tau_{i j}$ at the surface are accounted for in other parts of the formulation. In the earlier sections of this chapter, surface stress effects were seen to be accounted for by terms involving $\nabla \theta^{(k)}$. Therefore Eq. (2.33) can be written

$$
\frac{\partial u_{i}}{\partial t}+u_{j} \frac{\partial u_{i}}{\partial x_{j}}=-\frac{1}{\rho} \frac{\partial p}{\partial x_{i}}+g_{i}
$$

which is identical to Eq. (2.13) with $\mu=0$. 
Thus a suspension of rigid solid fragments in a turbulent fluid is a special case of the more general two-fluid case considered at the beginning of this chapter. A further special case contained in the theory is the problem of a single rigid body in a turbulent fluid. This will turn out to be a significant advantage of the present formulation when the problem of closure is considered in detail in the following chapter.

\section{References Cited}

Aris, R. 1962. Vectors, iensors and the basic equations of fuid mechanics. Englewood Cliffs: Prentice-Hall.

Lundgren, T. S. 1967. Distribution functions in the statistical theory of turbulence. Phys. Fluids. 10:969-975.

. 1969. Model equation for nonhomogeneous turbulence. Phys. Fluids. 12:485-497.

Maxey, M. R. and J. J. Riley. 1983. Equation of motion for a small rigid sphere in a nonuniform flow. Phys. Fluids. 26:883-889.

Oates, G. C. 1974. Vector Analysis. in C. E. Pearson, ed. Handbook of applied mathematics. New York: van Nostrand Reinhold.

Townsend, A. A. 2976. 2d ed. The structure of turbulent shear flow. Cambridge: Cambridge University Press. 


\section{Chapter III}

\section{Homogeneous Flow}

The goal of this chapter is to establish a point of departure for further closure modeling by taking advantage of the simplifying assumption of local homogeneity in the statistical field. "Local homogeneity" is used here in the same sense that "local equilibrium" is used in the kinetic theory of gases. It means that at any point the statistical field is independent of position, but that the average quantities may vary from point to point. (The assumption of isotropy will not be uniformly made in this chapter, for reasons that are explained as the developments unfold.)

Consideration of the case of homogeneous flow opens the way toward closure of the average equations derived in Chapter II. The closure problem is addressed in this chapter in three parts. The first part is concerned with terms that depend upon the one-point distribution function $f^{(k)}$, the second part is concerned with terms that depend upon the two-point distribution function $f_{1,2}^{(k, m)}$ and the third part deals with interface terms.

In addition to the assumption of local homogeneity, a variety of other approximations are required in order to complete the closure, especially with regard to the two-point terms. In this chapter the necessary approximations are made to the lowest order only, since the goal here is to illuminate the basic character of the one-point, two-point and interface terms. Here "lowest order" means approximations that are usually linear in $\left\langle\theta^{(1)}\right\rangle\left\langle\theta^{(2)}\right\rangle$ and, for the most part, free of mean flow gradients. Higher order approximations will be developed in the next chapter. All of the necessary approximations are motived by the physical principles of conservation, tensor invariance and Galilean invariance. To the extent possible, a universal formulation is attempted.

\section{ONE-POINT TERMS}

The lowest order estimate of the one-point terms will be obtained here by introducing the postulate that $f^{(k)}$ is Gaussian. The symbol $f_{(0)}^{(k)}$ will be used to distinguish the postulated distribution function from $f^{(k)}$ itself. The form for $f_{(0)}^{(k)}$ to be tested in this thesis is a two-material analog to the Gaussian form used by Lundgren (1969) for single-phase turbulence. The form selected is

$$
\begin{aligned}
f_{(0)}^{(k)}= & \delta(\alpha-1)\left\langle\theta^{(k)}\right\rangle\left(2 \pi q^{(k) 2}\right)^{-3 / 2} \exp -\left[\left(v_{i}-\left\langle u_{i}^{(k)}\right\rangle\right)^{2} / 2 q^{(k) 2}\right] \\
& +\delta(\alpha-0)\left\langle\theta^{(l)}\right\rangle\left(2 \pi q^{(l) 2}\right)^{-3 / 2} \exp -\left[\left(v_{i}-\left\langle u_{i}^{(l)}\right\rangle\right)^{2} / 2 q^{(l) 2}\right]
\end{aligned}
$$

where $l=3-k$, and $q^{(k) 2}$ is the mean square velocity fluctuation defined by 


$$
\frac{3}{2}\left\langle\theta^{(k)}\right\rangle q^{(k) 2}=\left\langle\frac{1}{2} C_{i}^{(k) 2} \theta^{(k)}\right\rangle
$$

Notice that this form has the required properties. It has the correct normalization

$$
\begin{aligned}
\int f_{(0)}^{(k)} d \mathbf{\Gamma}= & \frac{\left\langle\theta^{(k)}\right\rangle}{\left(2 \pi q^{(k) 2}\right)^{3 / 2}} \iint \delta(\alpha-1) e^{-\left(C_{i}^{(k) 2} / 2 q^{(k) 2}\right)} d \alpha d \mathbf{v} \\
& +\frac{\left\langle\theta^{(l)}\right\rangle}{\left(2 \pi q^{(l) 2}\right)^{3 / 2}} \iint \delta(\alpha-0) e^{-\left(C_{i}^{(l) 2} / 2 q^{(l) 2}\right)} d \alpha d \mathbf{v} \\
= & \left\langle\theta^{(k)}\right\rangle+\left\langle\theta^{(l)}\right\rangle \\
= & 1
\end{aligned}
$$

in which the notation $C_{i}^{(k)}=v_{i}-\left\langle u_{i}^{(k)}\right\rangle$ has been used. In Chapter II $C_{i}^{(k)}=$ $u_{i}-\left\langle u_{i}^{(k)}\right\rangle$ was used for the velocity fluctuation. In the developments that follow $C_{i}^{(k)}$ will always appear in an expectation expression or equivalently, inside an integral over $f_{(0)}^{(k)}$. In either case the meaning is the same, so there is no ambiguity.

Clearly $f_{(0)}^{(k)}$ has the proper $\alpha$-moment

$$
\begin{aligned}
\int \alpha f_{(0)}^{(k)} d \mathbf{\Gamma}= & \frac{\left\langle\theta^{(k)}\right\rangle}{\left(2 \pi q^{(k) 2}\right)^{3 / 2}} \iint \alpha \delta(\alpha-1) e^{-\left(C_{i}^{(k) 2} / 2 q^{(k) 2}\right)} d \alpha d \mathbf{v} \\
& +\frac{\left\langle\theta^{(k)}\right\rangle}{\left(2 \pi q^{(l) 2}\right)^{3 / 2}} \iint \alpha \delta(\alpha-0) e^{-\left(C_{i}^{(l) 2} / 2 q^{(l) 2}\right)} d \alpha d \mathbf{v} \\
= & \left\langle\theta^{(k)}\right\rangle
\end{aligned}
$$

the proper $\alpha \mathbf{v}$ - moment

$$
\begin{aligned}
\int \alpha \mathbf{v} f_{(0)}^{(k)} d \mathbf{\Gamma}= & \frac{\left\langle\theta^{(k)}\right\rangle}{\left(2 \pi q^{(k) 2}\right)^{3 / 2}} \iint \alpha \mathbf{v} \delta(\alpha-1) e^{-\left(C_{i}^{(k) 2} / 2 q^{(k) 2}\right)} d \alpha d \mathbf{v} \\
& +\frac{\left\langle\theta^{(k)}\right\rangle}{\left(2 \pi q^{(l) 2}\right)^{3 / 2}} \iint \alpha \mathbf{v} \delta(\alpha-0) e^{-\left(C_{i}^{(l) 2} / 2 q^{(l) 2}\right)} d \alpha d \mathbf{v} \\
= & \left\langle\theta^{(k)}\right\rangle\left\langle\mathbf{u}^{(k)}\right\rangle=\left\langle\theta^{(k)} \mathbf{u}\right\rangle,
\end{aligned}
$$

and the appropriate $\frac{1}{2} \alpha C_{i}^{(k) 2}-$ moment 


$$
\begin{aligned}
\int \alpha \frac{1}{2} C_{i}^{(k) 2} f_{(0)}^{(k)} d \Gamma= & \frac{\left\langle\theta^{(k)}\right\rangle}{\left(2 \pi q^{(k) 2}\right)^{3 / 2}} \iint \alpha \frac{1}{2} C_{i}^{(k) 2} \delta(\alpha-1) e^{-\left(C_{j}^{(k) 2} / 2 q^{(k) 2}\right)} d \alpha d \mathbf{v} \\
& +\frac{\left\langle\theta^{(l)}\right\rangle}{\left(2 \pi q^{(l) 2}\right)^{3 / 2}} \iint \alpha \frac{1}{2} C_{i}^{(l) 2} \delta(\alpha-0) e^{-\left(C_{j}^{(k) 2} / 2 q^{(l) 2}\right)} d \alpha d \mathbf{v} \\
= & \frac{3}{2}\left\langle\theta^{(k)}\right\rangle q^{(k) 2}=\left\langle\frac{1}{2} C_{i}^{(k) 2} \theta^{(k)}\right\rangle
\end{aligned}
$$

Notice that $f_{(0)}^{(k)}$ also contains the mean velocity, without $\alpha$-weighting. This is the mean velocity at a point with no reference to which material is at a point. Thus

$$
\begin{aligned}
\int \mathbf{v} f^{(k)} d \mathbf{T}= & \frac{\left\langle\theta^{(k)}\right\rangle}{\left(2 \pi q^{(k) 2}\right)^{3 / 2}} \iint \mathbf{v} \delta(\alpha-1) e^{-\left(C_{i}^{(k) 2} / 2 q^{(k) 2}\right)} d \alpha d \mathbf{v} \\
& +\frac{\left\langle\theta^{(l)}\right\rangle}{\left(2 \pi q^{(l) 2}\right)^{3 / 2}} \iint \mathbf{v} \delta(\alpha-0) e^{-\left(C_{i}^{(l) 2} / 2 q^{(l) 2}\right)} d \alpha d \mathbf{v} \\
= & \left\langle\theta^{(k)}\right\rangle\left\langle\mathbf{u}^{(k)}\right\rangle+\left\langle\theta^{(l)}\right\rangle\left\langle\mathbf{u}^{(l)}\right\rangle \\
= & \left\langle\mathbf{u} \theta^{(k)}\right\rangle+\left\langle\mathbf{u} \theta^{(l)}\right\rangle \\
= & \left\langle\mathbf{u}\left(\theta^{(k)}+\theta^{(l)}\right)\right\rangle \\
= & \langle\mathbf{u}\rangle
\end{aligned}
$$

which is a property that will be used in developing the exchange terms in Sec. 2 . With $f_{(0)}^{(k)}$ given by Eqs. (3.1) and (3.2) one can proceed to determine $R_{i j}^{(k)}$, $Q_{i}^{(k)}$ and the viscous work term. $R_{i j}^{(k)}$ is given by

$$
\begin{aligned}
R_{i j}^{(k)}= & -\left\langle C_{i}^{(k)} C_{j}^{(k)} \theta^{(k)}\right\rangle=-\int \alpha C_{i}^{(k)} C_{j}^{(k)} f_{(0)}^{(k)} d \mathbf{\Gamma} \\
= & -\frac{\left\langle\theta^{(k)}\right\rangle}{\left(2 \pi q^{(k) 2}\right)^{3 / 2}} \iint \alpha C_{i}^{(k)} C_{j}^{(k)} \delta(\alpha-1) e^{-\left(C_{k}^{(k) 2} / 2 q^{(k) 2}\right)} d x d \mathbf{v} \\
& -\frac{\left\langle\theta^{(l)}\right\rangle}{\left(2 \pi q^{(l) 2}\right)^{3 / 2}} \iint \alpha C_{i}^{(k)} C_{j}^{(k)} \delta(\alpha-0) e^{-\left(C_{k}^{(l) 2} / 2 q^{(l) 2}\right)} d \alpha d \mathbf{v} .
\end{aligned}
$$


The first diagonal component is

$$
\begin{aligned}
R_{11}^{(k)}=-\frac{\left\langle\theta^{(k)}\right\rangle}{\left(2 \pi q^{(k) 2}\right)^{3 / 2}}\{ & \int C_{1}^{(k) 2} e^{-\left(C_{1}^{(k) 2} / 2 q^{(k) 2}\right)} d C_{1} \\
& \cdot \int e^{-\left(C_{2}^{(k) 2} / 2 q^{(k) 2}\right)} d C_{2} \\
& \left.\cdot \int e^{-\left(C_{3}^{(k) 2} / 2 q^{(k) 2}\right)} d C_{3}\right\}
\end{aligned}
$$

where the subscripts clearly refer to the coordinate direction rather than evaluation at a particular point. Note that the integrals corresponding to cross products like $C_{1}^{(k)} C_{2}^{(k)}$ all vanish because they represent odd-powered integrals over a symmetric function that has limits extending from $-\infty$ to $+\infty$. Thus $R_{i j}^{(k)}=0$ for $i \neq j$. Completing the calculation for $R_{11}^{(k)}$ gives

$$
\begin{aligned}
R_{1 !}^{(k)} & =-\frac{\left\langle\theta^{(k)}\right\rangle}{\left(2 \pi q^{(k) 2}\right)^{3 / 2}}\left(2 \pi q^{(k) 6}\right)^{1 / 2}\left(2 \pi q^{(k) 2}\right)^{1 / 2}\left(2 \pi q^{(k) 2}\right)^{1 / 2} \\
& =-\left\langle\theta^{(k)}\right\rangle q^{(k) 2} .
\end{aligned}
$$

The results for $R_{22}^{(k)}$ and $R_{33}^{(k)}$ are the same. Therefore the Reynolds stress tensor is

$$
R_{i j}^{(k)}=-\left\langle\theta^{(k)}\right\rangle q^{(k) 2} \delta_{i j}
$$

of which only the diagonal part is nonzero. This is a consequence of the Gaussian form of the function $f_{(0)}^{(k)}$; the assumption imbedded in this choice of $f^{(k)}$ is the coordinate components of velocity at a point are uncorrelated. Therefore only what appears as a pressure emerges from $R_{i j}^{(k)}$. Hence the turbulent shear stress that corresponds to the off-diagonal elements of $R_{i j}^{(k)}$ is neglected by the current choice of $f_{(0)}^{(k)}$.

The energy fiux vector $Q_{j}^{(k)}$ is evaluated in a similar fashion. Thus

$$
\begin{aligned}
Q_{j}^{(k)} & =-\left\langle\frac{1}{2} C_{j}^{(k)} C_{i}^{(k) 2} \theta^{(k)}\right\rangle=-\frac{1}{2} \int \alpha C_{j}^{(k)} C_{i}^{(k) 2} f_{(0)}^{(k)} d \Gamma \\
& =-\frac{\left\langle\theta^{(k)}\right\rangle}{\left(2 \pi q^{(k) 2}\right)^{3 / 2}} \frac{1}{2} \int C_{j}^{(k)} C_{i}^{(k) 2} e^{-\left(C_{k}^{(k) 2} / 2 q^{(k) 2}\right)} d \mathrm{C}
\end{aligned}
$$

where the $\alpha$-integrals are already performed. Notice that this integral always contains an odd part, so that there is no coordinate direction for which a nonzero value is obtained. Therefore 


$$
Q_{j}^{(k)}=0
$$

The results so far can be interpreted by analogy to the kinetic theory of gases as representing the case of local equilibrium. In kinetic theory the assumption of local equilibrium means that at any point the distribution in molecular velocities has a Maxwell-Boltzmann form, but the mean square molecular velocity varies from point to point (Vincenti and Kruger 1965). With this assumption the mean flow equations for mass, momenturn and energy, called the Euler equations, are derived as moments of the Boltzmann equation. In the Euler equations, the stress tensor and heat flux vector are both zero. In the present case an analogous situation arises with respect to the turbulent shear stress tensor and tur bulent energy flux vector.

The term representing viscous work in the mean turbulence kinetic energy equation, is line (c) of Eq. (2.24). This can be written

$$
\begin{aligned}
\frac{\partial}{\partial x_{j}} \mu^{(k)}\left\langle C_{i}^{(k)} \theta^{(k)}\left(\frac{\partial C_{i}^{(k)}}{\partial x_{j}}+\frac{\partial C_{j}^{(k)}}{\partial x_{i}}\right)\right\rangle \\
=\frac{\partial}{\partial x_{j}} \mu^{(k)}\left\{\frac{1}{2}\left\langle\theta^{(k)} \frac{\partial}{\partial x_{j}} C_{i}^{(k)} C_{i}^{(k)}\right\rangle\right. \\
\left.\quad+\left\langle\theta^{(k)} \frac{\partial}{\partial x_{i}} C_{i}^{(k)} C_{j}^{(k)}\right\rangle-\left\langle\theta^{(k)} C_{j}^{(k)} \frac{\partial C_{i}^{(k)}}{\partial x_{i}}\right\rangle\right\},
\end{aligned}
$$

in which, for example

$$
\begin{aligned}
\left\langle\theta^{(k)} \frac{\partial}{\partial x_{j}} C_{i}^{(k)} C_{i}^{(k)}\right\rangle & =\frac{\partial}{\partial x_{j}} \int \alpha C_{i}^{(k)} C_{i}^{(k)} f^{(k)} d \Gamma \\
& =\frac{\partial}{\partial x_{j}} R_{i i}^{(k)}
\end{aligned}
$$

Notice that the incompressibility condition can be used to eliminate the last term

$$
\begin{aligned}
\left\langle\theta^{(k)} C_{j} \frac{\partial C_{i}^{(k)}}{\partial x_{i}}\right\rangle & =\left\langle\theta^{(k)} C_{j}^{(k)} \frac{\partial}{\partial x_{i}}\left(u_{i}-\left\langle u_{i}^{(k)}\right\rangle\right)\right\rangle \\
& =-\left\langle\theta^{(k)} C_{j}^{(k)}\right\rangle \frac{\partial}{\partial x_{i}}\left\langle u_{i}^{(k)}\right\rangle=0 .
\end{aligned}
$$

Thus the viscous work depends on $f^{(k)}$ through the Reynolds stress terms

$$
\frac{\partial}{\partial x_{j}} \mu^{(k)}\left\langle C_{i}^{(k)} \theta^{(k)}\left(\frac{\partial C_{i}^{(k)}}{\partial x_{j}}+\frac{\partial C_{j}^{(k)}}{\partial x_{i}}\right)\right\rangle
$$




$$
=\frac{\partial}{\partial x_{j}} \mu^{(k)}\left\{\frac{1}{2} \frac{\partial}{\partial x_{j}} R_{i i}^{(k)}+\frac{\partial}{\partial x_{j}} R_{i j}^{(k)}\right\} .
$$

This term is frequently negligible in comparison to the other one-point terms in single-fluid turbulent flows (Tennekes and Lumley 1972). The present theory is not yet sufficiently developed to allow general neglect of (3.5) so it will be retained until its magnitude can be compared to the other one- and two-point terms yet to be evaluated.

The term representing viscous dissipation is line (d) of Eq. (2.24). This onepoint term can best be evaluated in the present approximation as an integral over the two-point distribution function, in the limit as point two approaches point one. Thus

$$
\begin{gathered}
\mu^{(k)}\left\langle\theta^{(k)}\left(\frac{\partial C_{i}^{(k)}}{\partial x_{j}}+\frac{\partial C_{j}^{(k)}}{\partial x_{i}}\right) \frac{\partial C_{i}^{(k)}}{\partial x_{j}}\right\rangle \\
=\mu^{(k)} \lim _{r_{12} \rightarrow 0}\left\langle\theta_{1}^{(k)}\left(\frac{\partial C_{1 i}^{(k)}}{\partial x_{1 j}}+\frac{\partial C_{1 j}^{(k)}}{\partial x_{1 i}}\right) \frac{\partial C_{2 i}^{(k)}}{\partial x_{2 j}}\right\rangle \\
=\mu^{(k)} \lim _{r_{12} \rightarrow 0}\left\{\frac{\partial}{\partial x_{2 j}}\left\langle\theta_{1}^{(k)} \frac{\partial}{\partial x_{1 j}} C_{1 i}^{(k)} C_{2 i}^{(k)}\right\rangle+\frac{\partial}{\partial x_{2 j}}\left\langle\theta_{1}^{(k)} \frac{\partial}{\partial x_{1 i}} C_{1 j}^{(k)} C_{2 i}^{(k)}\right\rangle\right\} \\
=\mu^{(k)} \lim _{r_{12} \rightarrow 0}\left\{\frac{\partial^{2}}{\partial x_{1 j} \partial x_{2 j}} \int \alpha_{1} C_{1 i}^{(k)} C_{2 i}^{(k)} f_{1,2}^{(k, k)} d \mathbf{\Gamma}_{1,2}\right. \\
\left.\quad+\frac{\partial^{2}}{\partial x_{1 i} \partial x_{2 j}} \int \alpha_{1} C_{1 j}^{(k)} C_{2 i}^{(k)} f_{1,2}^{(k, k)} d \mathbf{\Gamma}_{1,2}\right\}
\end{gathered}
$$

where again $r_{12}=\left|x_{1}-x_{2}\right|$. Evaluation of expression (3.6) within the present approximation of locally homogeneous statistics will be postponed until the next section. This will provide the opportunity to establish a consistent manner by which two-point terms are to be handled in the initial approximation.

\section{TWO-POINT MOMENTUM TERMS}

This section considers the momentum exchange term that emerged in Chapter II, Sec. 3. This term can be broken into three parts that represent various features of the momentum exchange process, which is crucially dependent upon the two-point probability distribution function. The aim is to examine these parts and to extract from them low-order approximations that reflect their essential nature. 
The two-point term

$$
-\frac{\partial}{\partial x_{i}}\left\langle\theta^{(k)} p^{\prime}\right\rangle=-\frac{1}{4 \pi} \int \frac{\partial}{\partial x_{1 i}} \frac{1}{r_{12}} \frac{\partial^{2}}{\partial x_{2 j} \partial x_{2 k}} A_{j k}^{(k)} d \mathbf{x}_{2}
$$

was called the momentum exchange term in Chapter II, Sec. 3 , where

$$
A_{j k}^{(k)}=\sum_{m} \rho^{(m)} \int \alpha_{1} \alpha_{2} v_{2 j} v_{2 k}\left(f_{1,2}^{(k, m)}-f_{1}^{(k)} f_{2}^{(m)}\right) d \Gamma_{1,2}
$$

was referred to as a correlation coefficient, which is nonzero in the presence of a mean interpenetration, by the very nature of multimaterial flow.

The nature of Eq. (3.7) can be explored by first looking closely at $A_{i k}^{(k)}$. Evaluating the integrals in Eq. (3.8) yields

$$
A_{j k}^{(k)}=\sum_{m} \rho^{(m)}\left[\left\langle u_{2 j} u_{2 k} \theta_{2}^{(m)} \theta_{1}^{(k)}\right\rangle-\left\langle u_{2 j} u_{2 k} \theta_{2}^{(m)}\right\rangle\left\langle\theta_{1}^{(k)}\right\rangle\right] .
$$

Using $u_{2 j}=\left\langle u_{2 j}^{(k)}\right\rangle+C_{2 j}^{(k)}$ this can be rearranged to read

$$
\begin{aligned}
A_{j k}^{(k)}=\sum_{m} \rho^{(m)} & {\left[E^{(k, m)}\left\langle u_{2 j}^{(m)}\right\rangle\left\langle u_{2 k}^{(m)}\right\rangle\right.} \\
& +F_{j}^{(k, m)}\left\langle u_{2 k}^{(m)}\right\rangle+F_{k}^{(k, m)}\left\langle u_{2 j}^{(m)}\right\rangle \\
& \left.+G_{j k}^{(k, m)}\right],
\end{aligned}
$$

where

and

$$
\begin{aligned}
& E^{(k, m)}=\left\langle\theta_{1}^{(k)} \theta_{2}^{(m)}\right\rangle-\left\langle\theta_{1}^{(k)}\right\rangle\left\langle\theta_{2}^{(m)}\right\rangle, \\
& F_{j}^{(k, m)}=\left\langle C_{2 j}^{(m)} \theta_{2}^{(k)} \theta_{1}^{(k)}\right\rangle,
\end{aligned}
$$

$$
G_{j k}^{(k, m)}=\left\langle C_{2 j}^{(m)} C_{2 k}^{(m)} \theta_{2}^{(m)} \theta_{1}^{(k)}\right\rangle-\left\langle C_{2 j}^{(m)} C_{2 k}^{(m)} \theta_{2}^{(m)}\right\rangle\left\langle\theta_{1}^{(k)}\right\rangle
$$

This form of $A_{j k}^{(k)}$ facilitates its examination, especially in the case of homogeneous statistics. In this case the two-point distribution function $f_{1,2}^{(k, m)}$ is the same at all points $\mathbf{x}_{1}$ and varies only with the separation vector between points $\mathbf{r}_{12}=\mathbf{x}_{1}-\mathbf{x}_{2}$.

The idea that $f_{1,2}^{(k, m)}$ is intierently anisotropic for interpenetrating flow was established in Chapter I. The essential source of the anisotropy is relative motion of coherent masses of separated materials. Despite this basic property of multiphase flows, consider momentarily that the statistical field is also isotropic. This 
simplification, although quite unreal, is helpful in exposing the essential source of average momentum exchange in multiphase flow. The developments that unfold shortly show that a type of asymmetry in the statistical field is the main source of exchange effects.

If the statistical field is isotropic, then $f_{1,2}^{(k, m)}$ depends on the separation distance $\left|\mathbf{r}_{12}\right|=r_{12}$; a useful feature in examining $A_{j k}^{(k)}$, which depends on the two-point distribution function as shown in Eq. (3.8). With this, the parts of $A_{j k}^{(k)}$ given by the correlation coefficients $E^{(k, m)}, F_{j}^{(k, m)}$, and $G_{j k}^{(k, m)}$, can be modeled. Even though this modeling is by necessity now very crude, the exercise produces an appreciation for the nature of term (3.7) and gives a low-order approximation for it that posses certain essential physical properties.

To begin, notice that $E^{(1,1)}\left(r_{12}\right)$ at $r_{12}=0$ is, by definition in $(3.9 \mathrm{~b})$

$$
\begin{aligned}
E^{(1,1)}(0) & =\left\langle\theta_{1}^{(1)}\right\rangle-\left\langle\theta_{1}^{(1)}\right\rangle^{2} \\
& =\left\langle\theta_{1}^{(1)}\right\rangle\left(1-\left\langle\theta_{1}^{(1)}\right\rangle\right) \\
& =\left\langle\theta_{1}^{(1)}\right\rangle\left\langle\theta_{1}^{(2)}\right\rangle,
\end{aligned}
$$

and similarly

$$
E^{(1,2)}(0)=-\left\langle\theta_{1}^{(1)}\right\rangle\left\langle\theta_{1}^{(2)}\right\rangle .
$$

If points one and two are uncorrelated, then $\left\langle\theta_{1}^{(k)} \theta_{2}^{(m)}\right\rangle=\left\langle\theta_{1}^{(k)}\right\rangle\left\langle\theta_{2}^{(m)}\right\rangle$. Physically the correlation of two points can be expected to diminish with increasing separation, so that the points are completely uncorrelated as $r_{12} \rightarrow \infty$. Thus on physical grounds

$$
\lim _{r_{12} \rightarrow \infty} E^{(1,1)}=\left\langle\theta_{1}^{(1)}\right\rangle\left\langle\theta_{2}^{(1)}\right\rangle-\left\langle\theta_{1}^{(1)}\right\rangle\left\langle\theta_{2}^{(1)}\right\rangle=0
$$

and

$$
\lim _{r_{12} \rightarrow \infty} E^{(1,2)}=\left\langle\theta_{1}^{(1)}\right\rangle\left\langle\theta_{2}^{(2)}\right\rangle-\left\langle\theta_{1}^{(1)}\right\rangle\left\langle\theta_{2}^{(2)}\right\rangle=0 .
$$

Furthermore, $E^{(k, m)}\left(r_{12}\right)$ has an extremum at $r_{12}=0$. Using a technique employed by Hinze (1975 $2 \mathrm{~d}$ ed) for velocity correlation coefficients, this can be demonstrated by writing

$$
\begin{aligned}
\left\langle\left(\theta_{1}^{(1)}-\theta_{2}^{(1)}\right)^{2}\right\rangle & \geq 0 \\
\left\langle\theta_{1}^{(1) 2}-2 \theta_{1}^{(1)} \theta_{2}^{(1)}+\theta_{2}^{(1) 2}\right\rangle & \geq 0 \\
\left\langle\theta_{1}^{(1)}\right\rangle-2\left\langle\theta_{1}^{(1)} \theta_{2}^{(1)}\right\rangle+\left\langle\theta_{2}^{(1)}\right\rangle & \geq 0 \\
\left\langle\theta_{1}^{(1)}\right\rangle & \geq\left\langle\theta_{1}^{(1)} \theta_{2}^{(1)}\right\rangle
\end{aligned}
$$


where $\left\langle\theta_{1}^{(1)}\right\rangle=\left\langle\theta_{2}^{(1)}\right\rangle$ for a statistically homogeneous field. Subtracting $\left\langle\theta_{1}^{(1)}\right\rangle^{2}$ from both sides produces

$$
\left\langle\theta_{1}^{(1)}\right\rangle-\left\langle\theta_{1}^{(1)}\right\rangle^{2} \geq\left\langle\theta_{1}^{(1)} \theta_{2}^{(1)}\right\rangle-\left\langle\theta_{1}^{(1)} \theta_{2}^{(1)}\right\rangle
$$

or

$$
E^{(1,1)}(0) \geq E^{(1,1)}\left(r_{12}\right)
$$

as asserted. Also, one can easily show, for example,

$$
E^{(1,1)}=-E^{(1,2)}
$$

using the identity $\theta^{(1)}+\theta^{(2)}=1$.

The picture that is generated from the foregoing considerations is shown in Fig. 2, where $E^{(1,1)}$ is arbitrarily shown to take on negative values. (In general $E^{(1,1)}$ and $E^{(1,2)}$ could change signs, depending on the particular nature of the physical system being considered.)

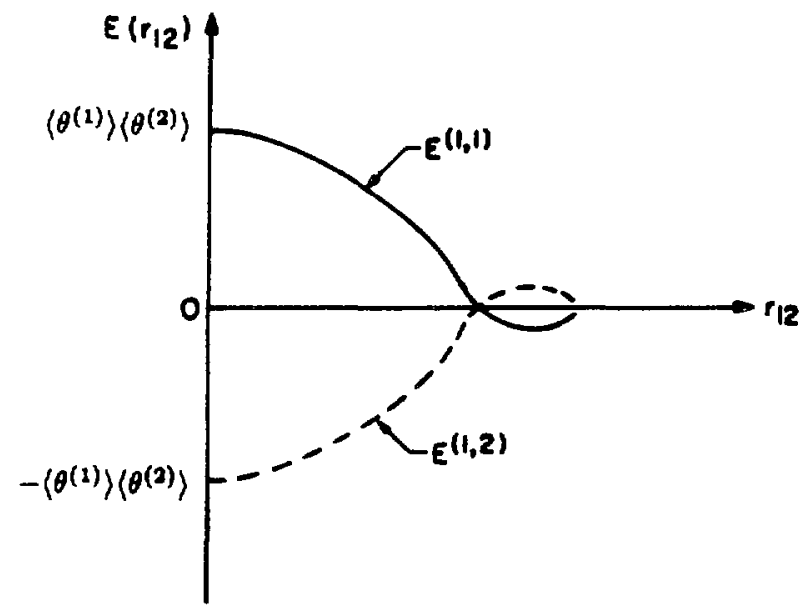

Figure 2. Sketch of the correlation coefficient $E\left(r_{12}\right)$.

Because $E^{(1,1)}=-E^{(1,2)}$, there is a single length scale over which these correlations tend substantially to zero. Surprisingly enough

$$
\begin{aligned}
E^{(1,1)} & =\left\langle\theta_{1}^{(1)} \theta_{2}^{(1)}\right\rangle-\left\langle\theta^{(1)}\right\rangle^{2} \\
& =\left\langle\left(1-\theta_{1}^{(2)}\right)\left(1-\theta_{2}^{(2)}\right)\right\rangle-\left(1-\left\langle\theta^{(2)}\right\rangle\right)^{2} \\
& =\left\langle\theta_{1}^{(2)} \theta_{2}^{(2)}\right\rangle-\left\langle\theta^{(2)}\right\rangle^{2} \\
& =E^{(2,2)},
\end{aligned}
$$


again because $\theta^{(1)}+\theta^{(2)}=1$ and $\left\langle\theta_{1}^{(2)}\right\rangle=\left\langle\theta_{2}^{(2)}\right\rangle$ in a homogeneous flow. Thus there is a single length scale over which the general coefficient $E^{(k, m)}$ tends substantially to zero.

On physical grounds, the overall magnitude of $E^{(k, m)}$ can be expected to diminish as either $\left\langle\theta^{(1)}\right\rangle$ or $\left\langle\theta^{(2)}\right\rangle$ becomes small. This is because the presence of the coefficient $A_{j k}^{(k, m)}$ is due completely to the finite probability of finding either of two materials at a point. As the probability of finding either material at a point becomes infinitesimal, so must the magnitude of $A_{j k}^{(k, m)}$.

Now comes the first leap into modeling approximations. Let the length $L$ represent the distance over which the coefficients $E^{(k, m)}$ remain significant. For example the scale $L$ would be the characteristic separation distance between entities in a uniform dispersion. (The correlation length $L$ plays the part of the mean free path in molecular dynamics.) One functional form that satisfies the limiting conditions on the $E^{(k, m)}$ coefficients is the exponential. Consider the forms

$$
\begin{aligned}
E^{(1,1)} & =\left\langle\theta_{1}^{(1)} \theta_{2}^{(1)}\right\rangle-\left\langle\theta^{(1)}\right\rangle\left\langle\theta^{(1)}\right\rangle \\
& \sim\left\langle\theta^{(1)}\right\rangle\left\langle\theta^{(2)}\right\rangle e^{-\left(r_{12} / L\right)^{2}}
\end{aligned}
$$

and

$$
\begin{aligned}
E^{(1,2)} & =\left\langle\theta_{1}^{(1)} e_{2}^{(2)}\right\rangle-\left\langle\theta^{(1)}\right\rangle\left\langle\theta^{(2)}\right\rangle \\
& \sim-\left\langle\theta^{(1)}\right\rangle\left\langle\theta^{(2)}\right\rangle e^{-\left(r_{12} / L\right)^{2}},
\end{aligned}
$$

each of which clearly satisfy the appropriate definitions at $r_{12}=0$, give the physically expected result for $r_{12} \rightarrow \infty$ and possess an extremum at $r_{12}=0$. Note too that all $E^{(k, m)}$ go to zero if either $\left\langle\theta^{(1)}\right\rangle$ or $\left\langle\theta^{(2)}\right\rangle$ vanish.

Consider now $F_{j}^{(k, m)}$, defined in Eq. (3.9c) whereby

$$
F_{j}^{(1,1)}(0)=\left\langle C_{j}^{(1)} \theta^{(1)}\right\rangle=\left\langle\left(u_{j}-\left\langle u_{j}^{(1)}\right\rangle\right) \theta^{(1)}\right\rangle=0
$$

and

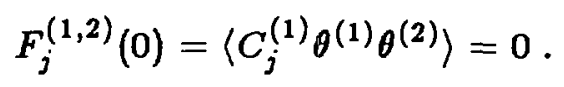

Again, points one and two can be expected to become statistically independent for large $r_{12}$ so that

$$
\lim _{r_{12} \rightarrow \infty} F_{j}^{(1,1)}=\left\langle C_{2 j}^{(1)} \theta_{2}^{(1)}\right\rangle\left\langle\theta_{1}^{(1)}\right\rangle=0
$$

and

$$
\lim _{r_{12} \rightarrow \infty} F_{j}^{(1,2)}=\left\langle C_{2 j}^{(2)} \theta_{2}^{(2)}\right\rangle\left\langle\theta_{1}^{(1)}\right\rangle=0 .
$$


The coefficient $F_{j}^{(k, m)}$ depends on the velocity fluctuation in addition to the material function at two points. The velocity can be expected to remain correlated to the material type for at most some multiple of an entity size scale $s$. Thus, in keeping with the single-length scale, exponential function approach to expressing the correlation coefficients, the magnitude of $F_{j}^{(k, m)}$ can be approximated by

$$
\left|\left\langle C_{j}^{(1)}\right\rangle\right|\left\langle\theta^{(1)}\right\rangle\left(\frac{r_{12}}{s}\right)^{2} e^{-\left(r_{12} / s\right)^{2}}
$$

For the direction of $F_{j}^{(k, m)}$ let us take the direction of $\left\langle C_{j}^{(k, m)}\right\rangle$ itself at point one. Hence

$$
F_{j}^{(1,1)}=\frac{\left\langle C_{j}^{(1)}\right\rangle}{\left|\left\langle C_{j}^{(1)}\right\rangle\right|}\left|\left\langle C_{j}^{(1)}\right\rangle\right|\left\langle\theta^{(1)}\right\rangle\left(\frac{r_{12}}{s}\right)^{2} e^{-\left(r_{12} / s\right)^{2}} .
$$

Now observe an interesting property of the vector $\left\langle C_{j}^{(1)}\right\rangle$. Notice the equality

$$
\begin{aligned}
\left\langle C_{j}^{(1)}\right\rangle & =\left\langle\left(u_{j}-\left\langle u_{j}^{(1)}\right\rangle\right)\right\rangle \\
& =\left\langle u_{j}\right\rangle-\left\langle u_{j}^{(1)}\right\rangle \\
& =\left\langle u_{j}\left(\theta^{(1)}+\theta^{(2)}\right)\right\rangle-\left\langle u_{j}^{(1)}\right\rangle \\
& =\left\langle u_{j} \theta^{(1)}\right\rangle+\left\langle u_{j} \theta^{(2)}\right\rangle-\left\langle u_{j}^{(1)}\right\rangle \\
& =\left\langle u_{j}^{(1)}\right\rangle\left\langle\theta^{(1)}\right\rangle+\left\langle u_{j}^{(2)}\right\rangle\left\langle\theta^{(2)}\right\rangle-\left\langle u_{j}^{(1)}\right\rangle \\
& =\left\langle u_{j}^{(1)}\right\rangle\left(1-\left\langle\theta^{(2)}\right\rangle\right)+\left\langle u_{j}^{(2)}\right\rangle\left\langle\theta^{(2)}\right\rangle-\left\langle u_{j}^{(1)}\right\rangle \\
& =\left\langle\theta^{(2)}\right\rangle\left(\left\langle u_{j}^{(2)}\right\rangle-\left\langle u_{j}^{(1)}\right\rangle\right) \\
& =\left\langle\theta^{(2)}\right\rangle\left\langle u_{j}^{(21)}\right\rangle
\end{aligned}
$$

where the shorthand notation for relative mean velocity $\left\langle u_{j}^{(2)}\right\rangle-\left\langle u_{j}^{(1)}\right\rangle=\left\langle u_{j}^{(21)}\right\rangle=$ $-\left\langle u_{j}^{(12)}\right\rangle$ is introduced. With this the foregoing form for $F_{j}^{(k, m)}$ becomes

$$
\begin{aligned}
F_{j}^{(1,1)} & =\left\langle C_{2 j}^{(1)} \theta_{2}^{(1)} \theta_{1}^{(1)}\right\rangle \\
& \sim\left\langle u_{j}^{(21)}\right\rangle\left\langle\theta^{(1)}\right\rangle\left\langle\theta^{(2)}\right\rangle\left(\frac{r_{12}}{s}\right)^{2} e^{-\left(r_{12} / s\right)^{2}} \\
F_{j}^{(1,2)} & =\left\langle C_{2 j}^{(2)} \theta_{2}^{(2)} \theta_{1}^{(1)}\right\rangle
\end{aligned}
$$




$$
\sim-\left\langle u_{j}^{(21)}\right\rangle\left\langle\theta^{(1)}\right\rangle\left\langle\theta^{(2)}\right\rangle\left(\frac{r_{12}}{s}\right)^{2} e^{-\left(r_{12} / s\right)^{2}}
$$

noting that these both satisfy the limits on $F_{j}^{(k, m)}$, go to zero if either $\left\langle\theta^{(1)}\right\rangle$ or $\left\langle\theta^{(2)}\right\rangle$ vanish, and diminish in magnitude with the relative mean velocity.

Now consider $G_{j k}^{(k, m)}$, which by definition has the properties at $r_{12}=0$

$$
\begin{aligned}
G_{j k}^{(1,1)}(0) & =\left\langle C_{j}^{(1)} C_{k}^{(1)} \theta^{(1)}\right\rangle-\left\langle C_{j}^{(1)} C_{k}^{(1)} \theta^{(1)}\right\rangle\left\langle\theta^{(1)}\right\rangle \\
& =-\left[R_{j k}^{(1)}-R_{j k}^{(1)}\left\langle\theta^{(1)}\right\rangle\right] \\
& =-R_{j k}^{(1)}\left(1-\left\langle\theta^{(1)}\right\rangle\right) \\
& =-R_{j k}^{(1)}\left\langle\theta^{(2)}\right\rangle
\end{aligned}
$$

and

$$
\begin{aligned}
G_{j k}^{(1,2)}(0) & =\left\langle C_{j}^{(2)} C_{k}^{(2)} \theta^{(2)} \theta^{(1)}\right\rangle-\left\langle C_{j}^{(2)} C_{k}^{(2)} \theta^{(2)}\right\rangle\left\langle\theta^{(1)}\right\rangle \\
& =R_{j k}^{(2)}\left\langle\theta^{(1)}\right\rangle
\end{aligned}
$$

$G_{j k}^{(k, m)}$ clearly goes to zero as points one and two become uncorrelated, as expected in the limit $r_{12} \rightarrow \infty$.

The correlation $G_{j k}^{(k, m)}$ depends on the square of the velocity fluctuation in addition to the material type at two points. Like $F_{j}^{(k, m)}, G_{j k}^{(k, m)}$ can be expected to remain nonzero over a distance corresponding to $s$ at most. The properties of $G_{j k}^{(k, m)}$ at $r_{12}=0$ show that this correlation contains the Reynolds stress. In the theory of single-fluid turbulence, a length scale called the integral scale is used to characterize the nonzero part of two-point velocity correlations. Here the analogous length scale $l$ will be defined as the length over which $G_{j k}^{(k, m)}$ tends substantially to zero. With this, the correlation modeling can continue. The forms for $G_{j k}^{(k, m)}$ to be considered are

$$
\begin{aligned}
G_{j k}^{(1,1)} & =\left\langle C_{2 j}^{(1)} C_{2 k}^{(1)} \theta_{2}^{(1)} \theta_{1}^{(1)}\right\rangle-\left\langle C_{2 j}^{(1)} C_{2 k}^{(1)} \theta_{2}^{(1)}\right\rangle\left\langle\theta_{1}^{(1)}\right\rangle \\
& \sim-R_{j k}^{(1)}\left\langle\theta^{(2)}\right\rangle e^{-\left(r_{12} / l\right)^{2}}
\end{aligned}
$$

and

$$
\begin{aligned}
G_{j k}^{(1,2)} & =\left\langle C_{2 j}^{(2)} C_{2 k}^{(2)} \theta_{2}^{(2)} \theta_{1}^{(1)}\right\rangle-\left\langle C_{2 j}^{(2)} C_{2 k}^{(2)} \theta_{2}^{(2)}\right\rangle\left\langle\theta_{1}^{(1)}\right\rangle \\
& \sim R_{j k}^{(2)}\left\langle\theta^{(1)}\right\rangle e^{-\left(r_{12} / l\right)^{2}}
\end{aligned}
$$


These forms also exhibit the appropriate properties at $r_{12}=0, r_{12} \rightarrow \infty$ and vanish for $\left\langle\theta^{(1)}\right\rangle$ or $\left\langle\theta^{(2)}\right\rangle$ going to zero. $\left(R_{j k}^{(1)}=-\left\langle C_{j}^{(1)} C_{k}^{(1)} \theta^{(1)}\right\rangle\right.$ goes to zero if $\theta^{(1)}=0$ everywhere.)

Expressions (3.10), and (3.12), and (3.13) can be inserted into Eq. (3.9a), producing a single expression approximating $A_{j k}^{(k)}$. The result for $k=1$ is

$$
\begin{aligned}
A_{j k}^{(1)}=\left\langle\theta^{(1)}\right\rangle\left\langle\theta^{(2)}\right\rangle & \left\{e^{-\left(r_{12} / L\right)^{2}}\left[\rho^{(1)}\left\langle u_{2 j}^{(1)}\right\rangle\left\langle u_{2 k}^{(1)}\right\rangle-\rho^{(2)}\left\langle u_{2 j}^{(2)}\right\rangle\left\langle u_{2 k}^{(2)}\right\rangle\right]\right. \\
& +\left(\frac{r_{12}}{s}\right)^{2} e^{-\left(r_{12} / \theta\right)^{2}}\left[\rho^{(1)}\left\langle u_{j}^{(21)}\right\rangle\left\langle u_{2 k}^{(1)}\right\rangle-\rho^{(2)}\left\langle u_{j}^{(21)}\right\rangle\left\langle u_{2 k}^{(2)}\right\rangle\right] \\
& +\left(\frac{r_{12}}{s}\right)^{2} e^{-\left(r_{12} / s\right)^{2}}\left[\rho^{(1)}\left\langle u_{2 j}^{(1)}\right\rangle\left\langle u_{k}^{(21)}\right\rangle-\rho^{(2)}\left\langle u_{2 j}^{(2)}\right\rangle\left\langle u_{k}^{(21)}\right\rangle\right] \\
& \left.-e^{-\left(r_{12} / l\right)^{2}}\left[\frac{\rho^{(1)}}{\left\langle\theta^{(1)}\right\rangle} R_{j k}^{(1)}-\frac{\rho^{(2)}}{\left\langle\theta^{(2)}\right\rangle} R_{j k}^{(2)}\right]\right\}
\end{aligned}
$$

Because of the symmetry property shown earlier (that is, $E^{(1,2)}=-E^{(1,1)}$ ) there follows the relation

$$
A_{j k}^{(1)}=-A_{j k}^{(2)}
$$

by inspection of (3.14). Expression (3.14) can in turn be placed into Eq. (3.7). Assuming that the average quantities remain approximately constant over the nonzero part of the exponentials, the result is

$$
\begin{aligned}
-\frac{\partial}{\partial x_{i}}\left\langle\theta^{(1)} p^{\prime}\right\rangle=-\left\langle\theta^{(1)}\right\rangle\left\langle\theta^{(2)}\right\rangle & \left\{\left[\rho^{(1)}\left\langle u_{j}^{(1)}\right\rangle\left\langle u_{k}^{(1)}\right\rangle-\rho^{(2)}\left\langle u_{j}^{(2)}\right\rangle\left\langle u_{k}^{(2)}\right\rangle\right] E_{i j k}^{\prime}\right. \\
& +\left\langle u_{j}^{(21)}\right\rangle\left[\rho^{(1)}\left\langle u_{k}^{(1)}\right\rangle-\rho^{(2)}\left\langle u_{k}^{(2)}\right\rangle\right] F_{i j k}^{\prime} \\
& \left.+\left\langle u_{k}^{(21)}\right\rangle \rho^{(1)}\left\langle u_{j}^{(1)}\right\rangle-\rho^{(2)}\left\langle u_{j}^{(2)}\right\rangle\right] F_{i j k}^{\prime} \\
& \left.-\left[\frac{\rho^{(1)}}{\left\langle\theta^{(1)}\right\rangle} R_{j k}^{(1)}-\frac{\rho^{(2)}}{\left\langle\theta^{(2)}\right\rangle} R_{j k}^{(2)}\right] G_{i j k}^{\prime}\right\}
\end{aligned}
$$

where

$$
\begin{aligned}
& E_{i j k}^{\prime}=\frac{1}{4 \pi} \int \frac{\partial}{\partial x_{1 i}} \frac{1}{r_{12}} \frac{\partial^{2}}{\partial x_{2 j} \partial x_{2 k}} e^{-\left(r_{12} / L\right)^{2}} d \mathrm{x}_{2}, \\
& F_{i j k}^{\prime}=\frac{1}{4 \pi} \int \frac{\partial}{\partial x_{1 i}} \frac{1}{r_{12}} \frac{\partial^{2}}{\partial x_{2 j} \partial x_{2 k}}\left(\frac{r_{12}}{s}\right)^{2} e^{-\left(r_{12} / s\right)^{2}} d \mathrm{x}_{2}
\end{aligned}
$$

and 


$$
G_{i j k}^{\prime}=\frac{1}{4 \pi} \int \frac{\partial}{\partial x_{1 i}} \frac{1}{r_{12}} \frac{\partial^{2}}{\partial x_{2 j} \partial x_{2 k}} e^{-\left(r_{12} / l\right)^{2}} d \mathbf{x}_{2}
$$

The assumption that the average quantities vary slowly over the nonzero part of the exponential functions corresponds to the requirements

$$
\begin{aligned}
& L \ll d \\
& s \ll d
\end{aligned}
$$

and

$$
l \ll d
$$

where $d$ is the scale of the particular physical system being considered.

The next step is to evaluate the integrals $E_{i j k}^{\prime}, F_{i j k}^{\prime}$, and $G_{i j k}^{\prime}$. This is most conveniently done in spherical coordinates, such as the system defined in Fig. 3.

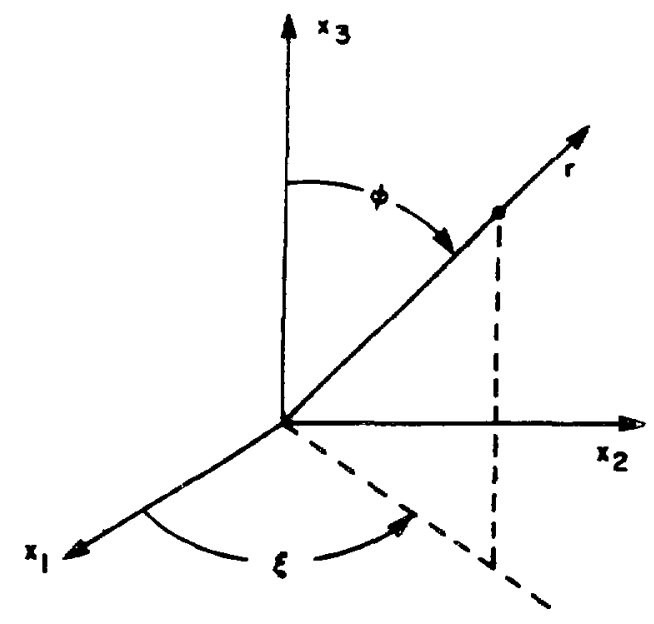

Figure 3. A spherical coordinate system.

With reference to Fig. 3 , where subscripts refer to coordinate directions, point one is the origin, point two is $x=x_{1} \hat{e}_{1}+x_{2} \hat{e}_{2}+x_{3} \hat{e}_{3}, r_{12}=r$,

$$
\begin{aligned}
& x_{1}=r \sin \phi \cos \xi \\
& x_{2}=r \sin \phi \sin \xi \\
& x_{3}=r \cos \phi,
\end{aligned}
$$


and the range of independent variables for the infinite space is $0 \leq \phi \leq \pi, \quad 0 \leq$ $\xi \leq 2 \pi, 0 \leq r \leq \infty$. Also, the element of volume is $d \mathbf{x}=r^{2} \sin \phi d \phi d \xi d r$. Performing the indicated integrations yields

$$
\begin{aligned}
& E_{i j k}^{\prime}=-\frac{(\pi)^{1 / 2}}{4 \pi L} \iint\left(\frac{x_{i}}{r} \frac{x_{j}}{r} \frac{x_{k}}{r}-\frac{\partial x_{k}}{\partial r}\right) \sin \phi d \phi d \xi=0 \\
& F_{i j k}^{\prime}=-\frac{1}{2} E_{i j k}^{\prime}\left(\frac{L}{s}\right)=0
\end{aligned}
$$

and

$$
G_{i j k}^{\prime}=E_{i j k}^{\prime}\left(\frac{L}{l}\right)=0 \text {. }
$$

Note thit $x_{i} / r$ and $\partial x_{k} / \partial r$ are independent of $r$, as seen in Eq. (3.18). These integrals vanish because the form assumed for the correlation coefficient $A_{j k}^{(k)}$ has no dependence on angle. This feature can be visualized by considering, for example, Eq. (3.16b) for $E_{i j k}^{\prime}$ rewritten with an arbitrary function $g(r, \xi, \phi)$ in place of the exponential

$$
E_{i j k}^{\prime}=\frac{1}{4 \pi} \int\left(\frac{\partial}{\partial x_{i}} \frac{1}{r}\right) \frac{\partial^{2}}{\partial x_{2 j} \partial x_{2 k}} g(r, \xi, \phi) d \mathbf{x} .
$$

Any nonlinear function $g$ that is not a function of the angles $\xi$ and $\phi$ will create an integrand whose contours of constant magnitude are spheres centered about the origin. A single contour of the magnitude of the integrand for this case is sketched in the $x_{1}-x_{2}$ plane in Fig. 4.

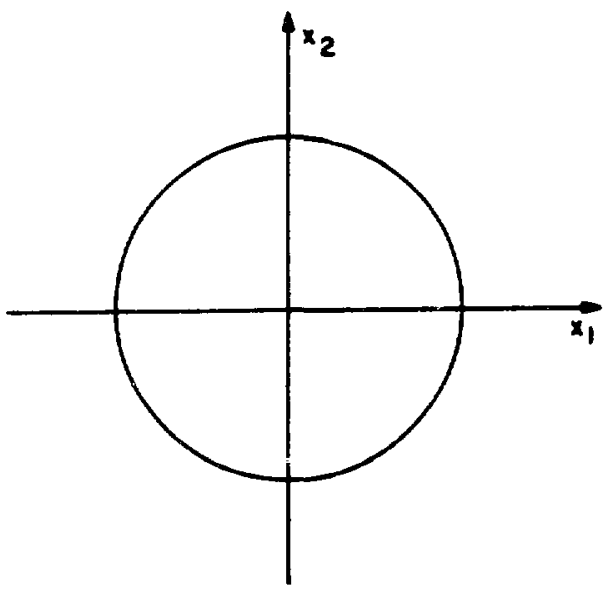

Figure 4. Constant magnitude plot for the integrand of Eq. (3.20). 
Now notice that

$$
\frac{\partial}{\partial x_{i}} \frac{1}{r}=-\frac{1}{r^{2}} \hat{e}_{r}
$$

where $\hat{e}_{r}$ is the unit radial vector. With reference to $F$ ig. 4 , it is clear that every point on the contour has a corresponding point on the opposite side where $\nabla\left(r_{12}^{-1}\right)$ has the opposite sign. The net result is a zero integral.

Recall the fundamental feature of anisotropy in multiphase flow. The probable velocity at a second point always depends on the directior of the vector $r_{12}$ and not just $\left|\mathbf{r}_{12}\right|$. Suppose now that $g(r, \xi, \phi)$ is an anisotropic function such that a constant magnitude contour plot of the integrand of Eq. (3.20) is given by Fig. 5 .

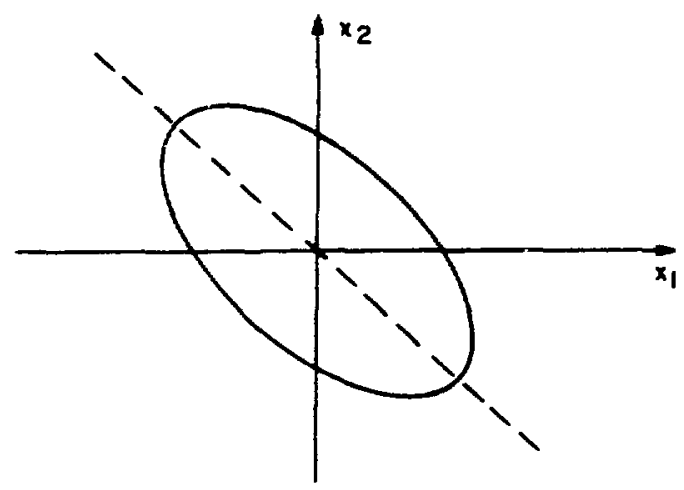

Figure 5. Constant magnitude plot for the integrand of Eq.(3.20) with an anisotropic function $\mathrm{g}$.

Figure 5 is a sketch of an ellipise centered at the origin, with its major axis lying at an arbitrary angle to the $x_{1}$ axis. The integral of this function is clearly zero, as it will be for any integrand that is symmetric about a plane passing through the origin. The only way that a nonzero integral will emerge is if there is an asymmetry in the integrand with respect to the origin, as shown in Fig. 6.

Hence the essential source of any nonzero exchange force between the two materials is asymmetry in the two-point statistics. Anisotropy alone cannot be responsible for the exchange effects.

The discuesion at the end of Chapter II concluded that the term $-\nabla\left\langle\theta^{(k)} p^{\prime}\right\rangle$ must be nonzero in the presence of average interpenetration. This conclusion and the foregoing considerations demand that the correlation coefficient $A_{j k}^{(k)}$ be modeled in a way that includes asymmetries in the statistical field in the presence of relative motion. 


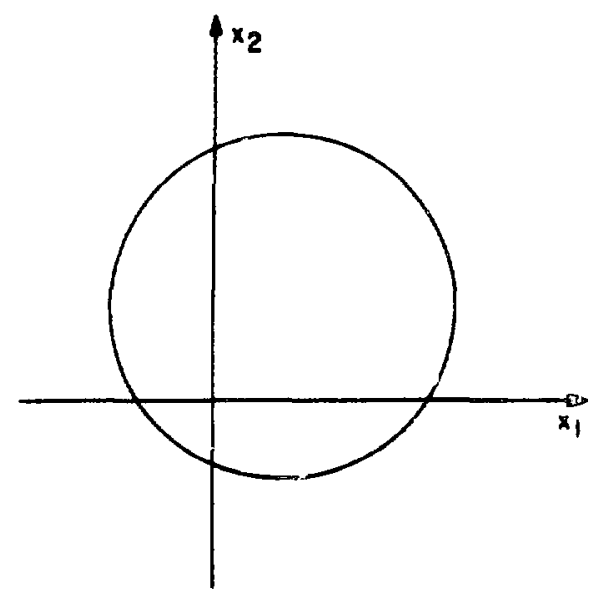

Figure 6. Constant magnitude plot for the integrand of Eq. (3.20) with an asymmetric function $\mathrm{g}$.

In order to model the terms $E_{i j k}^{\prime}, F_{i j k}^{\prime}$, and $G_{i j k}^{\prime}$ in a useful way, knowledge is required of the ways in which asymmetries in the statistical field can develop under various multimaterial flow circumstances. With reference to Chapter I, Sec. 3 , the force on a single rigid body submerged in an incompressible fluid is known to arise because of three basic effects: fluid rotation, viscous stress at the surface of the body and flow separation. (The force referred to is only that which is a consequence of the motion of the fluid relative to the body.) Since the statistical theory must contain the case of a single rigid body in an infinite fluid, it follows that asymmetries in the statistical field must arise as a result of the same basic effects. That the single body case must be contained by the statistical theory is quite evident. This is because the ensemble composed of a large number of identical members, each of which is an infinite fluid with a single submerged body, is a perfectly valid statistical entity.

The exchange force due to viscous stress at an interface between materials is accounted for by line (c) of Eq. (2.22), which was called the interfacial momenturn exchange term eariier on. One would suspect from this that the main sources of the asymmetry in the two-point statistics are fluid rotation and flow separaition.

Assuming that fluid rotation and flow separation are the dominant effects causing asymmetries in the statistical field, an estimate of the integral appearing in (3.19) can be constructed. One way to accomplish this is to add a quantity to the integrand of the symmetric case given by (3.19). The quantity added is to be considered a perturbation about the symmetric case that is caused by interpenetration. Therefore the perturbation parameter should become zero in the absence of relative motion. If the perturbation quantity is $a_{i j k}$, then (3.19) becomes 


$$
\begin{aligned}
E_{i j k}^{\prime} & =-\frac{(\pi)^{1 / 2}}{4 \pi L} \iint\left(\frac{x_{i}}{r} \frac{x_{j}}{r} \frac{x_{k}}{r}-\frac{\partial x_{k}}{\partial r}+a_{i j k}\right) \sin \phi d \phi d \xi \\
& =-\frac{1}{L} a_{i j k} \\
F_{i j k}^{\prime} & =\frac{1}{2 s} a_{i j k}
\end{aligned}
$$

and

$$
G_{i j k}^{\prime}=-\frac{1}{l} a_{i j k}
$$

where $a_{i j k}$ is considered to be constant with respect to the angles and a factor of $(\pi)^{1 / 2}$ is absorbed into the magnitude of $a_{i j k}$. Clearly the tensor $a_{i j k}$ is dimensionless. One form of the asymmetry perturbation $a_{i j k}$ will now be developed.

To lowest order, the principle direction of $a_{i j k}$ will be taken as the relative mean velocity, and the remaining directions given by $\delta_{j k}$. These directions are, for fluid 1

$$
\frac{\left\langle u_{i}^{(2)}\right\rangle-\left\langle u_{i}^{(1)}\right\rangle}{\left|\left\langle\mathbf{u}^{(2)}\right\rangle-\left\langle\mathbf{u}^{(1)}\right\rangle\right|} \delta_{j k}=\frac{\left\langle u_{i}^{(21)}\right\rangle}{\left|\left\langle\mathbf{u}^{(21)}\right\rangle\right|} \delta_{j k} .
$$

The magnitude of $a_{i j k}$ must depend upon the main effects causing the asymmetry: rotation and separation. Rotation can be manifested on a large scale by a bulk rotation in the mean field, or by smaller scale nonhomogeneities in the turbulence of the fluids. Both can be significant or insignificant depending on the particular problem. The effect of rotation on a small scale can be gauged by the inverse of a turbulent relative Reynolds number $R e_{t}^{-1}$ defined by

$$
R e_{t}^{-1}=\frac{[q] l}{\left|\left\langle\mathbf{u}^{(21)}\right\rangle\right| s},
$$

in which $[q]$ is a characteris tic measure of the turbulence given by

$$
[q]=\left\langle\theta^{(1)}\right\rangle q^{(1)}+\left\langle\theta^{(2)}\right\rangle q^{(2)} .
$$

Notice that the numerator is a turbulent viscosity based on the turbulence integral scale. The effect of rotation on a large scale is known to give rise to a lift force, such as the Saffman force. Because such forces depend upon gradients in the mean flow quantities, they will be regarded as higher-order effects and as such are to be neglected in the present low-order approximation.

The other physical source of statistical asyrnmetry is flow separation. In Chapter VI, Sec. 1, it is shown that the magnitude of the flow separation effect is 
properly accounted for by an inverse relative Reynolds number based on a turbulent viscosity involving the scale of the entity itself. Thus the ratio $[q] /\left\langle\left\langle\mathbf{u}^{(21)}\right\}\right|$ is used to gauge the flow separation effect.

Expressions (3.22) through (3.24) can be assembled into an approximation for the perturbation parameter, written for fluid one

$$
a_{i j k}=\left(\beta_{1} \frac{[q]}{\left|\left\langle\mathbf{u}^{(21)}\right\rangle\right|}+\beta_{2} R e_{t}^{-1}\right) \frac{\left\langle u_{i}^{(21)}\right\rangle}{\left|\left\langle\mathbf{u}^{(21)}\right\rangle\right|} \delta_{j k}
$$

where the nondimensional factors $\beta_{1}$ and $\beta_{2}$ are inserted before the inverse turbulent Reynolds numbers because the combined effects have been estimated on dimensional considerations alone.

Now expressions (3.25) and (3.21) can be placed into (3.15a) to yield

$$
\begin{aligned}
-\frac{\partial}{\partial x_{i}}\left\langle\theta^{(1)} p^{\prime}\right\rangle= & \left\langle\theta^{(1)}\right\rangle\left\langle\theta^{(2)}\right\rangle\left(\beta_{1} \frac{[q]}{\left|\left\langle\mathbf{u}^{(21)}\right\rangle\right|}+\beta_{2} R e_{t}^{-1}\right) \frac{\left\langle u_{i}^{(21)}\right\rangle}{\left|\left\langle\mathbf{u}^{(2-1)}\right\rangle\right|} \\
& \cdot\left\{\frac{1}{L}\left(\rho^{(1)}\left\langle u_{j}^{(1)}\right\rangle^{2}-\rho^{(2)}\left\langle u_{j}^{(2)}\right\rangle^{2}\right)\right. \\
& -\frac{1}{s}\left\langle u_{j}^{(21)}\right\rangle\left(\rho^{(1)}\left\langle u_{j}^{(1)}\right\rangle-\rho^{(2)}\left\langle u_{j}^{(2)}\right\rangle\right) \\
& \left.-\frac{1}{l}\left(\frac{\rho^{(1)}}{\left\langle\theta^{(1)}\right\rangle} R_{j j}^{(1)}-\frac{\rho^{(2)}}{\left\langle\theta^{(2)}\right\rangle} R_{j j}^{(2)}\right)\right\} .
\end{aligned}
$$

This expression violates two of the physical principles that were discussed at the beginning of this chapter. First, the expression is nonconservative of total momentum. That is to say, if the same expression with the material indices interchanged is added to (3.26) the result is nonzero. A second defect is that Eq. (3.26) is not invariant to a Galilean transformation. This means that if a fixed constant is added to the velocity, the term gives a different result for the change in momentum, which is physically incorrect. Equation (3.26) is an approximation to an expression that is both conservative of total momentum and invariant to a Galilean transformation. This means that the approximations introduced in obtaining Eq. (3.26) from Eq. (3.7) must be modified in a way that restores these essential physical properties. The first modification that is required is to remove the densities from the differences on the right side of Eq. (3.26). This can be accomplished by introducing a characteristic density $[\rho]$ defined by, say,

$$
[\rho]=\left\langle\theta^{(1)}\right\rangle \rho^{(1)}+\left\langle\theta^{(2)}\right\rangle \rho^{(2)}
$$

and multiplying each of the exponential expressions for the correlation coefficients in Eq. (3.9) by the factor 


$$
\frac{[\rho]}{\rho^{(m)}}
$$

This has the net effect of replacing the densities in (3.26) by $[\rho]$. (Notice that this definition of $[\rho]$ gives emphasis to the material property of the material most likely to be at a point.) Having done this, two more modifications to (3.26) are necessary. The first is the replacement

$$
\begin{aligned}
\left(\left\langle u_{j}^{(1)}\right\rangle^{2}-\left\langle u_{j}^{(2)}\right\rangle^{2}\right) & \rightarrow\left(\left\langle u_{j}^{(1)}\right\rangle-\left\langle u_{j}^{(2)}\right\rangle\right)^{2} \\
& =\left|\left\langle u^{(21)}\right\rangle\right|^{2}
\end{aligned}
$$

which is first-order accurate in velocity. The second modification is the replacement

$$
\begin{aligned}
\frac{R_{j j}^{(1)}}{\left\langle\theta^{(1)}\right\rangle}-\frac{R_{j j}^{(3)}}{\left\langle\theta^{(2)}\right\rangle} & =-3\left(q^{(1) 2}-q^{(2) 2}\right) \\
& \rightarrow-3\left|q^{(1) 2}-q^{(2) 2}\right|
\end{aligned}
$$

where Eq. (3.3) has been used for $R_{j j}^{(k)}$. The effect of (3.27) through (3.29) is to make (3.26) conservative of total momentum. The estimate (3.28) is necessary to ensure invariance to a Galilean transformation. The result of these alterations is

$$
\begin{aligned}
-\frac{\partial}{\partial x_{i}}\left\langle\theta^{(1)} p^{\prime}\right\rangle= & \left\langle\theta^{(1)}\right\rangle\left\langle\theta^{(2)}\right\rangle[\rho]\left(\beta_{1} \frac{[q]}{\left|\left\langle\mathbf{u}^{(21)}\right\rangle\right|}+\beta_{2} R e_{t}^{-1}\right)\left\langle u_{i}^{(21)}\right\rangle \\
& \cdot\left\{\frac{1}{L}\left|\left\langle\mathbf{u}^{(21)}\right\rangle\right|+\frac{1}{s}\left|\left\langle\mathbf{u}^{(21)}\right\rangle\right|+\frac{3}{l} \frac{\left|q^{(1) 2}-q^{(2) 2}\right|}{\left|\left\langle\mathbf{u}^{(21)}\right\rangle\right|}\right\},
\end{aligned}
$$

in which $L$ is the entity separation scale, $s$ is the entity size scale and $l$ is the integral scale for the turbulence. Notice that the entity separation scale $L$ may be approximated by

$$
L \sim \frac{s}{\left\langle\theta^{(*)}\right\rangle^{1 / 3}}
$$

where $\left\langle\theta^{(*)}\right\rangle$ is the volume fraction of the dispersed material. This states that the spacing $L$ becomes infinite if the probable presence of the dispersed material vanishes. Thus the first term in the brackets of (3.35) is proportional to $\left\langle\theta^{(1)}\right\rangle\left\langle\theta^{(2)}\right\rangle\left\langle\theta^{(*)}\right\rangle$ which means that in "thin" mixtures for which 


$$
\left\langle\theta^{(*)}\right\rangle \ll 1
$$

the $L^{-1}$ term is negligible. For this reason the $L^{-1}$ term in Eq. (3.30) will be neglected for now, since it clearly represents a higher-order effect. The $L^{-1}$ term will be further examined in Chapter IV. There it will be shown that this term accounts for asymmetries in the statistical field engendered by what can be called "collective effects", meaning the effects of more than one submerged body.

Notice that if

$$
\frac{\left|q^{(1) 2}-q^{(2) 2}\right|}{\left|\left\langle\mathrm{u}^{(2-1)}\right\rangle\right|^{2}} \ll \frac{l}{s}
$$

then the third term in the brackets of Eq. (3.30) is also negligible. The left side of (3.33) is like the square of a turbulence intensity. For many flows it may be expected that the integral scale $l$ is established by the entity size $s$, such that $l s^{-1} \sim 1$. In these cases (3.33) states that the $l^{-1}$ term of $(3.30)$ is negligible for cases in which the turbulence intensity is low. Since the estimates for the one-point terms can be considered valid only for low-intensity turbulence, the $1^{-1}$ term will be neglected from Eq. (3.30) in order to keep the present level of closure consistent. Another way to view this is that the $l^{-1}$ term is quadratic in the turbulence intensity. Therefore discussion of the $l^{-1}$ term is appropriately included in Chapter IV along with other higher-order effects.

For later convenience, Eq. (3.30) is expressed in the thin flow, low turbulence intensity case for material $k$ as

$$
-\frac{\partial}{\partial x_{i}}\left\langle\theta^{(k)} p^{\prime}\right\rangle=K^{*}\left(\left\langle u_{i}^{(l)}\right\rangle-\left\langle u_{i}^{(k)}\right\rangle\right)
$$

where

$$
K^{*}=[\rho]\left\langle\theta^{(1)}\right\rangle\left\langle\theta^{(2)}\right\rangle \frac{1}{s}\left(\beta_{1} \frac{[q]}{\left|\left\langle\mathbf{u}^{(21)}\right\rangle\right|}+\beta_{2} R e_{t}^{-1}\right)\left|\left\langle u^{(l)}\right\rangle-\left\langle\mathbf{u}^{(k)}\right\rangle\right|
$$

is a positive coefficient, and $l=3-k$ is the material index for the other material.

\section{TWO-POINT KINETIC ENERGY TERMS}

This section is concerned with the two-point terms appearing in the equation for fluctuational kinetic energy. The first of these is the term representing the rate at which the energy is dissipated into heat by action of viscous forces on a small scale. This term is a one-point term that was written in Sec. 1 of this chapter as a two-point term in the limit as point two approaches point one. Recalling Eq. (3.6) the dissipation rate was written 


$$
\begin{aligned}
& -\mu^{(k)}\left\langle\theta^{(k)}\left(\frac{\partial C_{i}^{(k)}}{\partial x_{j}}+\frac{\partial C_{j}^{(k)}}{\partial x_{i}}\right) \frac{\partial C_{i}^{(k)}}{\partial x_{j}}\right\rangle= \\
& -\mu^{(k)} \lim _{r_{12} \rightarrow 0}\left\{\frac{\partial^{2}}{\partial x_{1 j} \partial x_{2 j}}\left\langle\theta_{1}^{(k)} C_{1 j}^{(k)} C_{2 i}^{(k)}\right\rangle+\frac{\partial^{2}}{\partial x_{i j} \partial x_{2 j}}\left\langle\theta_{1}^{(k)} C_{1 j}^{(k)} C_{2 i}^{(k)}\right\rangle\right\}
\end{aligned}
$$

where $r_{12}=\left|\mathbf{x}_{1}-\mathbf{x}_{2}\right|$. Notice that this involves two-point correlation coefficients. In the limit $r_{12} \rightarrow 0$ these correlation coefficients are $-R_{i i}^{(k)}$ and $-R_{i j}^{(k)}$ respectively. Here the idea is to evaluate the dissipation term by modeling these correlation coefficients in a way similar to that used in the previous section. Consider for example

$$
\left\langle\theta_{1}^{(k)} C_{1 i}^{(k)} C_{2 i}^{(k)}\right\rangle=-R_{i i}^{(k)} e^{-(r / l)^{2}}
$$

and

$$
\left\langle\theta_{1}^{(k)} C_{1 j}^{(k)} C_{2 i}^{(k)}\right\rangle=-R_{j i}^{(k)} e^{-(r / l)^{2}}
$$

These forms satisfy the limit $r_{12} \rightarrow 0$ They also indicate that the correlations vanish as the two points become widely separated, which can be expected on physical grounds. The correlations in (3.38) and (3.39) also imply an isotropic statistical field.

Consider for the moment the fully homogeneous case so that $R_{i j}^{(k)}$ is independent of $x$. Also recall that because $r_{12}=x_{1}-x_{2}, \nabla_{1}=-\nabla_{2}$. In this approximation the right side of the dissipation term becomes

$$
\begin{aligned}
-\mu^{(k)} & \left\{R_{i i}^{(k)} \lim _{r_{12} \rightarrow 0} \frac{\partial^{2}}{\partial x_{1 j} \partial x_{1 j}} e^{-\left(r_{12} / l\right)^{2}}\right. \\
& \left.+R_{i j}^{(k)} \lim _{r_{12} \rightarrow 0} \frac{\partial^{2}}{\partial x_{1 i} \partial x_{1 j}} e^{-\left(r_{12} / l\right)^{2}}\right\} .
\end{aligned}
$$

The limits are calculated by performing the indicated differentiation and evaluating at $r_{12}=0$. Recognizing that the limits must be isotropic tensors, the limits are

$$
\begin{aligned}
\lim _{r_{12} \rightarrow 0} \frac{\partial^{2}}{\partial x_{1 j} \partial x_{1 j}} e^{-\left(r_{12} / l\right)^{2}} & =\left.\frac{\partial^{2}}{\partial r_{12}^{2}} e^{-\left(r_{12} / l\right)^{2}}\right|_{r_{12}=0} \delta_{j j} \\
& =-\left(\frac{2}{l^{2}}\right) \delta_{j j}
\end{aligned}
$$

and 


$$
\lim _{r_{12} \rightarrow 0} \frac{\partial^{2}}{\partial x_{1 j} \partial x_{1 j}} e^{-\left(r_{12} / l\right)^{2}}=-\left(\frac{2}{l^{2}}\right) \delta_{i j} .
$$

With these the. viscous dissipation term becomes

$$
\begin{aligned}
-\mu^{(k)}\left\langle\theta^{(k)}\left(\frac{\partial C_{i}^{(k)}}{\partial x_{j}}+\frac{\partial C_{j}^{(k)}}{\partial x_{i}}\right) \frac{\partial C_{i}^{(k)}}{\partial x_{j}}\right\rangle & =2 \frac{\mu^{(k)}}{l^{2}}\left\{3 R_{i i}^{(k)}+R_{i j}^{(k)} \delta_{i j}\right\} \\
& =8 \frac{\mu^{(k)}}{l^{2}} R_{i i}^{(k)} \\
& =-24 \frac{\mu^{(k)}}{l^{2}}\left\langle\theta^{(k)}\right\rangle q^{(k) 2} .
\end{aligned}
$$

Note that in the last step Eq. (3.3) has been used for $R_{i i}^{(k)}$, and $l$ is the integral scale of the turbulence correlation coefficients. The foregoing result is analogous to one for a single turbulent fluid obtained by a variety of other workers, for example Tennekes and Lumley (1972).

In the remainder of this section the two-point terms containing the pressure fluctuation will be considered. The first of these is the diffusion flux of energy due to pressure fluctuations, $\left\langle C_{i}^{(k)} \theta^{(k)} p^{\prime}\right\rangle$. With the expression for $p^{\prime}$ from the previous section, Eq. (3.7), it can be seen that $\left\langle C_{i}^{(k)} \theta^{(k)} p^{\prime}\right\rangle$ will involve triple velocity correlations. If the two point distribution function $F_{1,2}^{(k, m)}$ is joint normal, the triple correlations will vanish. Therefore to be consistent with the treatment of one point terms, $\left\langle C_{i}^{(k)} \theta^{(k)} p^{\prime}\right\rangle$ will be neglected in the first approximation.

The next two-point energy term is the multimaterial turbulence creation term $-\left\langle\mathbf{C}^{(k)}\right\rangle \cdot \nabla\left\langle\theta^{(k)} p^{\prime}\right\rangle$, which is easily evaluated using the results of Sec. 2. Thus

$$
-\left\langle C_{i}^{(k)}\right\rangle \frac{\partial}{\partial x_{i}}\left\langle\theta^{(k)} p^{\prime}\right\rangle=a^{(k)} K^{*}\left\langle u_{i}^{(l k)}\right\rangle^{2},
$$

where Eqs. (3.11), (3.55), and (3.36) have been used. Since $K^{*}$ is a positive coefficient, the expression in (3.40) is clearly positive. This means that any average relative motion will result in a positive contribution to the fluctuational energy of both materials.

Notice that $K^{*}\left\langle u_{i}^{(l k)}\right\rangle^{2}$ is the total rate of fluctuational energy production due to interpenetrating motion. This is the rate at which the ordered kinetic energy is transfered into disordered energy. Equation (3.40) says that a fraction $a^{(k)}$ of this goes into material $k$. Evaluating (3.40) exactly, according to the results of Sec. 2, implys $a^{(k)}=\left\langle\theta^{(l)}\right\rangle$. By analogy to the case of molecular mixtures, it can be expected that this is not completely correct. This is because a similar term appears in the molecular mixture case that is proportioned according to a mass ratio (Braginskii 1965). The relevant mass ratio is determined on the basis of the 
detailed nature of a single collision between two molecules of the different species. If $m_{1}$ and $m_{2}$ are the masses of molecule types one and two respectively, then the fraction of fluctuational energy production going to species one is

$$
\frac{m_{2}}{m_{1}+m_{2}} \text {. }
$$

Hence if species one is very light compared to species two, most of the ordered energy that is taken from the mean flow appears in the chaotic energy of the lighter material.

Derivation of a similar result for the interpenetrating flow case requires replacing the collision between two molecules with, say, the idea of an "interaction" between an isolated dispersed entity, and a mass of fluid. Whereas the mass of the isolated entity is well-defined, the proper mass of fluid encountered in a "collison" with the entity is not yet defined. If $V^{(1)}$ and $V^{(2)}$ are the respective volumes of the continuous material and the dispersed material, then the fractions become

$$
a^{(1)}=\frac{\rho^{(2)}}{\gamma \rho^{(1)}+\rho^{(2)}}
$$

and

$$
a^{(2)}=\frac{\gamma \rho^{(1)}}{\gamma \rho^{(1)}+\rho^{(2)}}
$$

by analogy to Eq. (3.41), where

$$
\gamma=\frac{V^{(1)}}{V^{(2)}}
$$

is the ratio of the "interaction" volume of the continuous material to that of a dispersed entity. This does not determine the proper volume of fluid, it merely provides an additional unknown. In the applications that appear in Chapter VI, it is found that the ratio in volumes can, at least provisionally, be represented by an expression that equates to a statement of "pressure" equilibrium for the turbulent interpenetrating motion.

The last two-point energy term considered in this section is $\nabla \cdot\left\langle\mathbf{C}^{(k)}\right\rangle\left\langle\theta^{(k)} p^{\prime}\right\rangle$. With Eq. (3.7), this can be written

$$
\begin{aligned}
& \frac{\hat{\vartheta}}{\partial x_{i}}\left\langle C_{i}^{(k)}\right\rangle\left\langle\theta^{(k)} p^{\prime}\right\rangle \\
& \quad=\frac{1}{4 \pi} \frac{\partial}{\partial x_{i}}\left\langle C_{i}^{(k)}\right\rangle \int \frac{1}{r_{12}} \frac{\partial^{2}}{\partial x_{2 j} \partial x_{2 k}} A_{j k}^{(k)} d \mathrm{x}_{2} \\
& \quad=\frac{1}{4 \pi} \int \frac{\partial}{\partial x_{1 i}} \frac{1}{r_{12}} \frac{\partial^{2}}{\partial x_{2 j} \partial x_{2 k}}\left\langle C_{1 i}^{(k)}\right\rangle A_{j k}^{(k)} d \mathrm{x}_{2}
\end{aligned}
$$


where $A_{j k}^{(k)}$ is given by Eq. (3.8). Like the pressure-velocity diffusion term, this involves triple correlations and will be neglected as a higher-order effect.

So far a term representing the conservative exchange in fluctuational energy has not explicitly been isolated. Physically such a term can be expected to appear. Further, the rate of this exchange should be independent of any mean relative motion. This is because a body held motionless in a turbulent fluid, when released will itself begin to fluctuate even if there is no mean motion.

The fact that the foregoing type of exchange term is not, at this stage, retained means that it must have been imbedded in one of the neglected terms. This does not prove to be a serious defect in the equations, as will be seen in later chapters. This merely means that the term is negligible for the particular problems considered. However, it should be noted that in general the missing energy exchange term may not be negligible.

\section{INTERFACE TERMS}

Now the interface terms appearing in Eq. (2.22) for the mean momentum and Eq. (2.24) for the mean fluctuational kinetic energy are considered. These are

$$
\left\langle\left(p^{\prime} \delta_{i j}-\tau_{i j}\right)\right\rangle \frac{\partial \theta^{(k)}}{\partial x_{j}}
$$

and

$$
\left\langle\left(\mu^{\prime} \delta_{i j}-\tau_{i j}\right) C_{i}^{(k)}\right\rangle \frac{\partial \theta^{(k)}}{\partial x_{j}}
$$

respectively. The goal is to obtain approximations to these terms that are consistent with the low-order estimates derived in the previous three sections.

In Chapter II, Sec. 4 the viscous parts of these interface terms were expressed in an integral form. There it was realized that the distribution functions do not contain statistical information concerning the interface structure. This prevents the expression of terms (3.43) and (3.44) as integrals over the distribution functions, so that the interface terms must be handled in a more heuristic fashion. Fortunately the results of the foregoing sections provide clues on how to proceed.

To begin, the pressure fluctuation part of the interface terms will be considered negligible in comparison to the total stress part. The rationale is that, in the low-order approximation, attention is limited to cases for which the effects of fluctuations are small.

Now, consider first the term (3.43) from the momentum equation. Like the term $-\nabla\left\langle\theta^{(k)} p^{\prime}\right\rangle,(3.43)$ is a momentum exchange term. In Chapter II, Sec. 4 the viscous part of (3.43) was written in integral form for an arbitrary volume $V$. The expression is 


$$
-\frac{1}{V} \int_{V}\left\langle\tau_{i j} \frac{\partial \theta^{(k)}}{\partial x_{j}}\right\rangle d V=-\frac{1}{V} \int_{S}\left\langle\tau_{i j} \hat{n}_{j}^{(k)}\right\rangle d s,
$$

where the integral on the right is over the surface contained in $V$ with normal $\hat{\mathbf{n}}^{(k)}$ directed away from material $k$. Here the expression is divided by $V$ so that the right side has the appearance of a volume average of an ensemble average. On dimensional grounds the right side of (3.53) will be approximated by a characteristic stress times a characteristic surface area per unit volume with the direction established by the mean relative velocity. Thus

$$
-\left\langle\tau_{i j} \frac{\partial \theta^{(k)}}{\partial x_{j}}\right\rangle \sim \alpha\left\{\frac{[\rho][\nu]\left(\left\langle u_{i}^{(l)}\right\rangle-\left\langle u_{i}^{(k)}\right\rangle\right)}{s}\right\} \frac{\left\langle\theta^{(l)}\right\rangle\left\langle\theta^{(k)}\right\rangle}{s}
$$

where the bracketed part is the stress combined with the direction, the last part estimates the area per unit volume and $\alpha$ is a coefficient of order unity. By analogy to the characteristic density $[\rho]$, the characteristic viscosity is defined

$$
[\nu]=\left\langle\theta^{(1)}\right\rangle \nu^{(1)}+\left\langle\theta^{(2)}\right\rangle \nu^{(2)} \text {. }
$$

The coefficient $\alpha$ can be determined for the special case of widely dispersed spheres moving slowly in a fluid. If the relative motion is slow enough, Stoke's result, Eq. (1.3), can be invoked to obtain $\alpha=9 / 2$ in that case.

The interface term (3.46) can be rearranged in terms of the inverse viscous relative Reynolds number $R e_{v}^{-1}$ defined by

$$
R e_{v}^{-1}=\frac{[\nu]}{\left|\left\langle\mathbf{u}^{(l k)}\right\rangle\right| s}
$$

and combined with the pressure part of the momentum exchange term. The result is

$$
-\left\langle\tau_{i j} \frac{\partial \theta^{(k)}}{\partial x_{j}}\right\rangle-\frac{\partial}{\partial x_{j}}\left\langle\theta^{(k)} p^{\prime}\right\rangle=K\left(\left\langle u^{(l)}\right\rangle-\left\langle u^{(k)}\right\rangle\right)
$$

in which

$$
K=[\rho]\left\langle\theta^{(1)}\right\rangle\left\langle\theta^{(2)}\right\rangle \frac{1}{s}\left(\beta_{1} \frac{[q]}{\left|\left\langle\mathbf{u}^{(21)}\right\rangle\right|}+\beta_{2} R e_{t}^{-1}+\frac{9}{2} R e_{v}^{-1}\right)\left|\left\langle\mathbf{u}^{(l k)}\right\rangle\right|
$$

where the Stoke's limit value of $\alpha$ is used, mainly as a means of insuring that a proper limiting behavior will be obtained from the expression. 


\section{SUMMARY}

This section is a summary and discussion of the results from Sections 1 through 3 of this chapter. For this purpose, the conservation equations for homogeneous flow are written for $k=1,2$ and $l=2,1$. These are the mean mass conservation equation

$$
\rho^{(k)}\left[\frac{\partial}{\partial t}\left\langle\theta^{(k)}\right\rangle+\frac{\partial}{\partial x_{j}}\left\langle u_{j}^{(k)}\right\rangle\left\langle\theta^{(k)}\right\rangle\right]=0,
$$

the mean momentum conservation equation

$$
\begin{aligned}
& \rho^{(k)}\left[\frac{\partial}{\partial t}\left\langle u_{i} \theta^{(k)}\right\rangle+\frac{\partial}{\partial x_{j}}\left\langle u_{j}^{(k)}\right\rangle\left\langle u_{i} \theta^{(k)}\right\rangle\right] \\
&=-\left\langle\theta^{(k)}\right\rangle \frac{\partial}{\partial x_{i}}\langle p\rangle+\rho^{(k)}\left\langle\theta^{(k)}\right\rangle g_{i} \\
&+\frac{\partial}{\partial x_{j}}\left[\mu^{(k)}\left\langle\theta^{(k)}\right\rangle D_{i j}^{(k)}-\rho^{(k)}\left\langle\theta^{(k)}\right\rangle q^{(k)} \delta_{i j}\right] \\
&+K\left(\left\langle u_{i}^{(l)}\right\rangle-\left\langle u_{i}^{(k)}\right\rangle\right),
\end{aligned}
$$

and the conservation equation for mean fluctuational energy

$$
\begin{aligned}
\rho^{(k)}\left[\frac{\partial}{\partial t}\left\langle\frac{1}{2} C_{i}^{(k) 2} \theta^{(k)}\right\rangle+\frac{\partial}{\partial x_{j}}\left\langle u_{j}^{(k)}\right\rangle\right. & \left.\left\langle\frac{1}{2} C_{i}^{(k) 2} \theta^{(k)}\right\rangle\right] \\
= & -\rho^{(k)}\left\langle\theta^{(k)}\right\rangle \frac{3}{2} q^{2} \frac{\partial\left\langle u_{i}^{(k)}\right\rangle}{\partial x_{i}} \\
& +\frac{5}{2} \frac{\partial}{\partial x_{j}} \mu^{(k)}\left\langle\theta^{(k)}\right\rangle q^{(k)} \\
& -24 \frac{\mu^{(k)}}{l^{2}}\left\langle\theta^{(k)}\right\rangle q^{(k)} \\
& +a^{(k)} K\left(\left\langle u_{i}^{(l)}\right\rangle-\left\langle u_{i}^{(k)}\right\rangle\right)^{2} .
\end{aligned}
$$

Equations (3.52), (3.53), and (3.54) are three equations in the four primary unknowns $\left\langle\theta^{(k)}\right\rangle,\left\langle u_{i} \theta^{(k)}\right\rangle,\left\langle\frac{1}{2} C_{i}^{(k) 2} \theta^{(k)}\right\rangle$, and $\langle p\rangle$. The fourth equation needed to close the set is

$$
\left\langle\theta^{(1)}\right\rangle+\left\langle\theta^{(2)}\right\rangle=1
$$


The auxiliary variables, all defined in terms of the primary unknowns, are the mean material velocity

$$
\left\langle\mathbf{u}^{(k)}\right\rangle=\frac{\left\langle\theta^{(k)} \mathbf{u}\right\rangle}{\left\langle\theta^{(k)}\right\rangle}
$$

the mean material rate of strain

$$
D_{i j}^{(k)}=\frac{\partial}{\partial x_{j}}\left\langle u_{i}^{(k)}\right\rangle+\frac{\partial}{\partial x_{i}}\left\langle u_{i}^{(k)}\right\rangle .
$$

the root mean square material velocity fluctuation $q^{(k)}$

$$
\frac{3}{2}\left\langle\theta^{(k)}\right\rangle q^{(k) 2}=\left\langle\frac{1}{2} C_{i}^{(k) 2} \theta^{(k)}\right\rangle
$$

the momentum ex hange coefficient

$$
K=[\rho]\left\langle\theta^{(1)}\right\rangle\left\langle\theta^{(2)}\right\rangle \frac{\mathrm{I}}{s}\left(\beta_{1} \frac{\mid q]}{\left|\left\langle\mathbf{u}^{(21)}\right\rangle\right|}+\beta_{2} R e_{t}^{-1}+\frac{9}{2} R e_{v}^{-1}\right)\left|\left\langle\mathbf{u}^{(l k)}\right\rangle\right|
$$

the inverse viscous relative Reynolds number

$$
\operatorname{Re}_{v}^{-1}=\frac{|\nu|}{\left|\left\langle\mathbf{u}^{(21)}\right\rangle\right| s}
$$

the inverse turbulent relative Reynolds number

$$
R e_{t}^{-1}=\frac{[q] l}{\left|\left\langle\mathbf{u}^{(21)}\right\rangle\right| s}
$$

the composite density

$$
[\rho]=\left\langle\theta^{(1)}\right\rangle \rho^{(1)}+\left\langle\theta^{(2)}\right\rangle \rho^{(2)}
$$

the composite kinematic viscosity

$$
[\nu]=\left\langle\theta^{(1)}\right\rangle \nu^{(1)}+\left\langle\theta^{(2)}\right\rangle \nu^{(2)}
$$

and the compcsite root mean square velocity fluctuation

$$
[q]=\left\langle\theta^{(1)}\right\rangle q^{(1)}+\left\langle\theta^{(2)}\right\rangle q^{(2)} .
$$

The coefficients $\beta_{1}$ and $\beta_{2}$ are dimensionless and of order unity. These are to be determined empirically. The length scales $s$ and $l$ are the entity size and turbulence integral scale respectively. For a suspension of rigid particles $s$ is prescribed and is the characteristic size of the particles. For bubble or droplet flow, $s$ is the scale of the bubbles or droplets. 
Notice that $s$ can be considered to be a function of space and time by use of an experimental correlation. One example, for the case of bubble or droplet flows, is given by the Weber number discussed in Chapter I. Using Eq. (1.7), an estimate for such flows is given by

$$
\frac{s \rho^{*}}{\sigma}\left|\left\langle\mathbf{u}^{(21)}\right\rangle\right|=10
$$

where $\rho^{*}$ is the constant density of the continuous material and $\sigma$ is the interfacial surface tension. The continuous material in a bubbly flow is essentially the one with the largest volume fraction (so that $\rho^{*} \sim[\rho]$ ).

In the case of a suspension of solids that fragment and coalesce, or solids that tend to clump together, a similar treatment can be used. Consider, for example,

$$
s=n(\mathbf{x}, t) s_{0}
$$

where $n(\mathbf{x}, t)$ is the number of solid pieces of scale $s_{0}$ in a clump. Here $n$ is to be determined from experimental data that provide a correspondence between $n$ and the other average quantities. Thus the theory is not strictly limited to a single entity size.

The only remaining quantity yet to be made precise is the turbulence integral scale $l$. A variety of choices exist for estimating $l$ as a function of the other mean flow quantities. These have been reviewed by Rodi (1980) for the single incompressible fluid case, and discussed briefly here in Chapter I, Sec. 4. For the moment the selection of a way to determine $l$ will be postponed until the next chapter. There the goal is to develop a multimaterial analog of the single material $k-\epsilon$ turbulence theory, where the scale $l$ is contained in the turbulence energy dissipation rate $\epsilon$.

\section{References Cited}

Lundgren, T. S. 1967. Distribution functions in the statistical theory of turbulence. Phys. Fluids. 10:969-975.

1969. Model equation for nonhomogeneous turbulence. Phys. Fluids. 12:485-497.

Tennekes, H. and J. L. Lumley. 1972. A first course in turbulence. Cambridge: The MIT Press.

Vincenti, W. G. and C. H. Kruger. 1965. Introduction to physical gas dynamics. New York: John Wiley \& Sons. 


\section{Chapter IV}

\section{Nonhomogeneous Flow}

This chapter extends the results of Chapter III to the case of nonhomogeneous flow. The focus here is first on the single-point terms that were represented in the previous chapter using a Gaussian form for the probability distribution function $f^{(k)}$. The first main geal is to develop representations for the Reynolds stress, energy flux, and pressure work terms, for the multimaterial case, that reflect the general non-Gaussian nature of $f^{(k)}$. The second goal is to find a plausible nonhomogeneous representation for the momentum exchange function.

In Sec. 1 a multimaterial $k-\epsilon$ theory is developed. There the approach is to utilize the analogy between single-fluid turbulence theory and multimaterial turbulence theory that has been established in Chapters II and III. Thus the approach is somewhat heuristic and can benefit from being placed on a firm theoretical foundation. This is shown to be possible in Sec. 2, which contains a brief discussion of how the multimaterial $k-\epsilon$ theory can be connected to more fundamental principles. In Sec. 2 the equations developed in Sec. 1 are shown to be connected to the relaxation-time theory of Lundgren (1969).

In Sec. 3 attention is redirected to the momentum exchange term. There the effect of statistical nonhomogeneity is included in a way designed to be consistent with the approximations imbedded in the $k-\epsilon$ theory for $R_{i j}^{(k)}$. The goal is to make the nonhomogeneous extension in a way that exposes the tensoriai character of the momentum exchange coefficient. Section 3 also revisits the part of the momentum exchange force involving differences in the fluctuational velocity. In this case, the nonhomogeneous extension leads to a force analogous to the so-called thermal force in a molecular mixture of different species.

\section{MULTIMATERIAL $k-\epsilon$ THEORY}

The multimaterial $k-\epsilon$ theory developed here follows by direct analogy to the single fluid $k-\epsilon$ theory discussed in Chapter I. There are five steps in this development. These are:

1. Introduction of an expression for the multimaterial Reynolds stress $R_{i j}^{(k)}$, containing the turbulence viscosity $\nu_{t}^{(k)}$ for each material.

2.Relation of the turbulence kinetic energy $\left\langle k^{(k)}\right\rangle$ and integral scale $l^{(k)}$ to $\nu_{t}^{(k)}$, for each material.

3. Relation of the integral scale to $\left\langle k^{(k)}\right\rangle$ and the dissipation rate $\left\langle\epsilon^{(k)}\right\rangle$ for each fluid.

4. Representation of the diffusive terms in the energy equation using a turbulent Prandtl number. 
5. Representation of the $\left\langle\epsilon^{(k)}\right\rangle$ evolution by analogy to the evolution of the turbulence kinetic energy.

As in the previous chapters, the development will first be followed through for the case of two incompressible fluids. The case of solid fragments suspended in an ircunpressible fluid then follows as a subset of the two-fluid case.

$\mathrm{T}$ c begin, recall the Reynolds stress per unit mass for material $k$, defined by

$$
R_{i j}^{(k)}=-\left\langle\theta^{(k)} C_{i}^{(k)} C_{j}^{(k)}\right\rangle
$$

where $C_{i}^{(k)}=u_{i}-\left\langle u_{i}^{(k)}\right\rangle$. Now let $\left\langle k^{(k)}\right\rangle$ be the mean turbulence kinetic energy per unit mass for material $k$. Thus*

$$
\left\langle k^{(k)}\right\rangle=\left\langle\frac{1}{2} C_{i}^{(k) 2} \theta^{(k)}\right\rangle
$$

whose evolution equation is given by Eq. (3.24). The appropriate generalization of Eq. (1.36) for the Reynolds stress is then

$$
R_{i j}^{(k)}=-\frac{2}{3}\left\langle k^{(k)}\right\rangle \delta_{i j}+\nu_{t}^{(k)}\left\langle\theta^{(k)}\right\rangle\left[D_{i j}^{(k)}-\frac{2}{3} \frac{\partial\left\langle u_{k}^{(k)}\right\rangle}{\partial x_{k}} \delta_{i j}\right]
$$

where, as before, the deformation rate tensor is

$$
D_{i j}^{(k)}=\frac{\partial\left\langle u_{i}^{(k)}\right\rangle}{\partial x_{j}}+\frac{\partial\left\langle u_{j}^{(k)}\right\rangle}{\partial x_{i}} .
$$

Equation (4.3) defines the kinematic turbulence viscosity for material $k, \nu_{t}^{(k)}$.

The next step relates $\nu_{t}^{(k)}$ to $\left\langle k^{(k)}\right\rangle$ and the integral scale $l^{(k)}$ in a fashion identical to Eq. (1.37). This is

$$
\nu_{t}^{(k)}=\frac{C_{\mu}^{\prime}\left\langle k^{(k)}\right\rangle^{1 / 2} l^{(k)}}{\left\langle\theta^{(k)}\right\rangle^{1 / 2}} .
$$

Now the integral scale is eliminated in favor of the dissipation rate $\left\langle\epsilon^{(k)}\right\rangle$, defined

$$
\left\langle\epsilon^{(k)}\right\rangle=\frac{1}{2} \nu^{(k)}\left\langle\theta^{(k)}\left(\frac{\partial C_{i}^{(k)}}{\partial x_{j}}+\frac{\partial C_{j}^{(k)}}{\partial x_{i}}\right)^{2}\right\rangle,
$$

which is approximated by

* Note that this could equally well be defined $\left\langle\theta^{(k)}\right\rangle\left\langle k^{(k)}\right\rangle=\left\langle\frac{1}{2} C_{i}^{(k) 2} \theta^{(k)}\right\rangle$, which would enable writing the left side of (3.24) using the Lagrangian derivative. The form selected here is used because $\left\langle k^{(k)}\right\rangle$ defined by $(4.2)$ is a conserved quantity. 
by analogy to Eq. (1.38).

$$
\left\langle\epsilon^{(k)}\right\rangle=C_{D} \frac{\left\langle k^{(k)}\right\rangle^{3 / 2}}{l^{(k)}\left\langle\theta^{(k)}\right\rangle^{3 / 2}}
$$

Expressions (4.5) and (4.7) combine to yield

$$
\nu_{t}^{(k)}=C_{\mu} \frac{\left\langle k^{(k)}\right\rangle^{2}}{\left\langle\theta^{(k)}\right\rangle\left\langle\epsilon^{(k)}\right\rangle},
$$

where $C_{\mu}=C_{\mu}^{\prime} C_{D}$ is a new coefficient that is to be determined experimentally.

The diffusive parts of Eq. (3.24) are now to be modeled following the approximation of Eq. (1.42). This produces

$$
\left\langle\frac{1}{2} C_{j}^{(k)} C_{i}^{(k) 2} \theta^{(k)}\right\rangle+\left\langle C_{j}^{(k)} \theta^{(k)} p^{\prime}\right\rangle=\frac{\nu_{t}^{(k)}}{\sigma_{k}} \frac{\partial\left\langle k^{(k)}\right\rangle}{\partial x_{j}} .
$$

Placing expression (4.3), (4.6), and (4.9) into Eq. (3.24) yields

$$
\begin{aligned}
\frac{\partial\left\langle k^{(k)}\right\rangle}{\partial t}+\frac{\partial}{\partial x_{j}}\left\langle u_{j}^{(k)}\right\rangle\left\langle k^{(k)}\right\rangle & =\frac{\partial}{\partial x_{j}} \frac{\nu_{t}^{(k)}}{\sigma_{k}} \frac{\partial\left\langle k^{(k)}\right\rangle}{\partial x_{j}} \\
& +\left[P^{(k)}-\left\langle\epsilon^{(k)}\right\rangle+\frac{a^{(k)}}{\rho^{(k)}} F_{i}^{(k)}\left\langle u_{i}^{(l k)}\right\rangle\right]
\end{aligned}
$$

from which the viscous diffusion term has been neglected, the mean flow production rate $P^{(k)}$ is defined

$$
P^{(k)}=R_{i j}^{(k)} \frac{\partial\left\langle u_{i}^{(k)}\right\rangle}{\partial x_{j}}
$$

and $\left\langle\epsilon^{(k)}\right\rangle$ is the mean dissipation rate for material $k$. Equation (3.2) can be used to relate the mean velocity fluctuation $q^{(k)}$ to $\left\langle k^{(k)}\right\rangle$. This is

$$
\frac{3}{2}\left\langle\theta^{(k)}\right\rangle q^{(k) 2}=\left\langle\frac{1}{2} C_{i}^{(k) 2} \theta^{(k)}\right\rangle
$$

The evolution equation for the dissipation rate $\left\langle\epsilon^{(k)}\right\rangle$ is written in the same spirit as Eq. (1.45), with two extensions. These will be discussed shortly. Consider the equation

$$
\begin{aligned}
& \frac{\partial\left\langle\epsilon^{(k)}\right\rangle}{\partial t}+\frac{\partial}{\partial x_{j}}\left\langle u_{j}^{(k)}\right\rangle\left\langle\epsilon^{(k)}\right\rangle=\frac{\partial}{\partial x_{j}} \frac{\nu_{t}^{(k)}}{\sigma_{\epsilon}} \frac{\partial\left\langle\epsilon^{(k)}\right\rangle}{\partial x_{j}}+2\left\langle\theta^{(k)}\right\rangle[\nu] \nu^{(k)}\left(\frac{\partial^{2}\left\langle u_{i}^{(k)}\right\rangle}{\partial x_{j} \partial x_{k}}\right)^{2} \\
& +\frac{\left\langle\epsilon^{(k)}\right\rangle}{\left\langle k^{(k)}\right\rangle}\left[C_{1} P^{(k)}-C_{2}\left\langle\epsilon^{(k)}\right\rangle\right]+C_{3} C_{\mu} \frac{a^{(k) 3 / 2}}{\rho^{(k)}} \frac{\left|\left\langle\mathbf{u}^{(21)}\right\rangle\right|}{s} F_{i}^{(k)}\left\langle u_{i}^{(i k)}\right\rangle
\end{aligned}
$$


in which $\sigma_{\epsilon}$ is the ratio of the turbulent viscosity to the diffusion coefficient for turbulence dissipation rate. Here $C_{1}, C_{2}$ and $C_{3}$ are coefficients of order unity, which, like $\sigma_{\epsilon}$ and $\sigma_{k}$, are to be determined from experiments.

The first extension in modeling the $\left\langle\epsilon^{(k)}\right\rangle$ equation is the use of the composite viscosity in the second term on the right. This is done so that added dissipation is produced in regions of flow where the curvature in velocity profile is great, even if material $k$ is a field of solid fragments. The second extension is the use of an inverse time scale other than

$$
\frac{\left\langle\epsilon^{(k)}\right\rangle}{\left\langle k^{(k)}\right\rangle}
$$

for modeling the rate of $\left\langle\epsilon^{(k)}\right\rangle$ production due to the interpenetration creation term. The reason for using something proportional to

$$
\frac{\left|\left\langle\mathbf{u}^{(21)}\right\rangle\right|}{s}
$$

instead, is to insure the appropriate rate of $\left\langle\epsilon^{(k)}\right\rangle$ creation due to interpenetrating flow. The remaining factors are present only because of the favorable results given by their presence in the applications of Chapter VI.

Equations (4.3), (4.8), and (4.10-13) are a multimaterial $k-\epsilon$ model for determining the Reynolds stress $R_{i j}^{(k)}$. Together with appropriate initial and boundary conditions, these equations are to be used with the equations for mean mass and momentum conservation for examining the dynamics of complex turbulent multimaterial flows. In order to utilize the foregoing theory with confidence, a set of values for the coefficients $C_{\mu}, \sigma_{k}, \sigma_{\epsilon}$, and $C_{1}-C_{3}$ must be found. In this thesis the approach is empirical, whereby values are sought that provide the best correspondence to experimental data in a wide range of industrial conditions. The starting place is of course the single-fluid values for $C_{\mu}, \sigma_{k}, \sigma_{\epsilon}, C_{1}$, and $C_{2}$. These are listed in Eqs. (1.46a-e). The new coefficient introduced in this theory is $C_{3}$, which will be taken as unity for applications of Chapter VI.

From the practical standpoint of engineering calculations, the foregoing result is satisfactory for many purposes. From the scientific viewpoint however, the heuristic approach is less satisfying because the results are not necessarily universal. This situation can be remedied in part by the more fundamental considerations that are contained in the next section. Before going forward, however, some comments are required concerning the multimaterial $k-\epsilon$ theory in the case of solid fragments in an incompressible fluid.

The physical picture of fluctuational energy loss by viscous dissipation in the small-scale motion is satisfying, provided the two materials are both fluids. Another form of dissipation must be present, however, if one of the materials is a field of dispersed solid fragments. It is easy to conceive of a significant loss in the translational kinetic energy of individual fragments through a collisional 
mechanism. In this the process of particle-particle impacts results in the transfer of energy from a translational mode to one of rotation. The use of the $k-\epsilon$ approach for a particulate field requires such a mechanism to be effective.

\section{CONNECTION TO LUNDGREN'S THEORY}

Here the discussion turns to the matter of establishing a fundamental basis for the approximations leading to the multimaterial $k-\epsilon$ theory introduced in the previous section. This can be approached in at least two different ways, for the single-fluid case, neither of which yield complete results in their present stage of development. The first is due to Lundgren (1969) who showed an important connection between the evolution equation for the velocity probability distribution function and the turbulent viscosity formulation used in $k-\epsilon$ single-fluid theory. The second is called Renormalization Group Analysis ( Yakhot and Orszag 1986). The goal of this section is to provide a brief look at how the $k-\epsilon$ theory can, at least in part, be established on fundamental grounds. The further development of the formalism, by whatever method, remains as a significant research project.

The first main result that Lundgren (1967) obtained was to show the existence of an infinite hierarchy of equations for the statistics of a single, incompressible, turbulent fluid. The next step was to model the equation for the single point velocity distribution function $f$ (Lundgren 1969), using a single-relaxation-time approach, so that an approximate solution for $f$ could be obtaired. This approximate solution provides an essential basis for the $k-\epsilon$ theory, and will be displayed here after providing the basic connection between the present multimaterial theory and the theory of Lundgren.

The first step is the derivation of the evolution equation for the multimaterial probability distribution function $f^{(k)}(\mathbf{x}, \mathbf{v}, \alpha, t)$ defined in Chapter II, and written

$$
f^{(k)}=\left\langle\delta\left(\theta^{(k)}-\alpha\right) \delta(\mathbf{u}-\mathbf{v})\right\rangle .
$$

Again, $f^{(k)} d \alpha d v$ is the probability that the material function $\alpha$ is in $\alpha+d \alpha$ and the velocity $\mathbf{v}$ is in $\mathbf{v}+d \mathbf{v}$ at position $\mathbf{x}$ and time $t$. The material function $\theta^{(k)}$ satisfies

$$
\frac{\partial \theta^{(k)}}{\partial t}+u_{j} \frac{\partial \theta^{(k)}}{\partial x_{j}}=0
$$

by definition, and the velocity at a point satisfies

$$
\frac{\partial u_{i}}{\partial t}+u_{j} \frac{\partial u_{i}}{\partial x_{j}}=-\frac{1}{\rho} \frac{\partial p}{\partial x_{i}}+\frac{1}{\rho} \frac{\partial \tau_{i j}}{\partial x_{j}} \quad, .
$$

The equation for $f^{(k)}$ is obtained by differentiating Eq. (4.16) and using Eqs. (4.17) and (4.18) to eliminate time derivatives of $\theta^{(k)}$ and $u$. The result is 


$$
\begin{aligned}
& \frac{\partial f^{(k)}}{\partial t}+\frac{\partial}{\partial x_{j}} v_{j} f^{(k)}-\frac{1}{\rho^{(k)}} \frac{\partial\langle p\rangle}{\partial x_{i}} \frac{\partial f^{(k)}}{\partial v_{i}} \\
& =\frac{1}{\rho^{(k)}} \frac{\partial}{\partial v_{i}}\left\langle\delta\left(\theta^{(k)}-\alpha\right) \delta(\mathbf{u}-\mathbf{v}) \frac{\partial}{\partial x_{j}}\left(p^{\prime} \delta_{i j}-\tau_{i j}\right)\right\rangle
\end{aligned}
$$

where $p^{\prime}=p-\langle p\rangle$. As shown in Chapter II, Sec. 4, the right side of (4.19) can be written in terms of integrals over the two-point distribution function $f_{1,2}^{(k, m)}$. Note that the average equations derived in Chapter II, correspond to the $\alpha, \alpha \mathbf{v}$ and $\frac{1}{2} \alpha(\mathbf{v}-\langle\mathbf{u}\rangle)^{2}$ moments of Eq. (4.19).

The next step in establishing a fundamental basis for a multimaterial $k-\epsilon$ theory is to approximate the right side of (4.19) in such a way that the essential results of Chapter III are retained. Since Eq. (4.19) is new, as are the results of Chapter III, such an approximation does not yet exist. Therefore, to ma.ke the point of this section, consideration is shifted to Lundgren's model equation for the single- fluid distribution function $f$.

The model equation proposed by Lundgren (1969) is

$$
\begin{aligned}
\frac{\partial f}{\partial t}+\frac{\partial}{\partial x_{j}} v_{j} f-\frac{1}{\rho} \frac{\partial\langle p\rangle}{\partial x_{i}} & \frac{\partial f}{\partial v_{i}} \\
& =-\frac{1}{\tau}\left(f-f_{0}\right)+\frac{1}{3} \frac{\epsilon}{q^{2}} \frac{\partial}{\partial v_{i}}\left(v_{i}-u_{i}\right) f,
\end{aligned}
$$

where $\tau^{-1}$ is an inverse relaxation time given by

$$
\frac{1}{\tau}=\frac{4(\epsilon+D k / D t)}{q^{2}}
$$

in which the coefficient 4 is expected to be universal, and $\epsilon, k$ and $q$ are respectively the dissipation rate, turbulence kinetic energy and root mean square velocity fluctuation. Also $D / D t$ is the Lagrangian operator

$$
\frac{D}{D t}=\frac{\partial}{\partial t}+\left\langle u_{j}\right\rangle \frac{\partial}{\partial x_{j}},
$$

and $f_{0}$ is, with $\mathbf{C}=\mathbf{v}-\langle\mathbf{u}\rangle$,

$$
f_{0}=\frac{1}{\left(2 \pi q^{2}\right)^{3 / 2}} \mathrm{e}^{-\left(C_{i}^{2} / 2 q^{2}\right)}
$$

so that the kinetic energy and root mean square velocity fluctuation are connected by 


$$
\frac{3}{2} q^{2}=k
$$

A key element of Lundgren's work is the perturbation solution to Eq. (4.20) for $f$ close to $f_{0}$. The result is

$$
f=f_{0}\left\{1-\frac{\nu_{t}}{q^{2}}\left[\left(\frac{1}{2} \frac{C_{j}^{2}}{q^{2}}-\frac{5}{2}\right) \frac{C_{i}}{q^{2}} \frac{\partial q^{2}}{\partial x_{i}}+\frac{C_{i} D_{i j} C_{j}}{2 q^{2}}\right]\right\}
$$

where $D_{i j}$ is the mean deformation rate

$$
D_{i j}=\frac{\partial\left\langle u_{i}\right\rangle}{\partial x_{j}}+\frac{\partial\left\langle u_{j}\right\rangle}{\partial x_{i}}
$$

and the coefficient $\nu_{t}$ is

$$
\nu_{t}=q^{2} \tau .
$$

The approximate result for $f$ enables computation of the Reynolds stress $R_{i j}$ and the energy flux vector $Q_{i}$. These are

$$
\begin{aligned}
R_{i j} & =-\int C_{i} C_{j} f d \mathbf{C} \\
& =-\frac{3}{2} k \delta_{i j}+\nu_{t} D_{i j}
\end{aligned}
$$

and

$$
\begin{aligned}
Q_{i} & =-\int \frac{1}{2} C_{i} C_{j}^{3} f d \mathrm{C} \\
& =-\frac{5}{3} \nu_{t} \frac{\partial k}{\partial x_{i}}
\end{aligned}
$$

Equation (4.27) is identical to that of standard single-fluid $k-\epsilon$ theory. Equation (4.28) is similar in form to the diffusion approximation used for energy flux and pressure work diffusion, with $\sigma_{k}=3 / 5$. This value is low compared to Launder and Spalding's (1974) value of $\sigma_{k}=1.0$ because (4.28) does not contain the pressure work term. Combining Eqs. (4.21) and (4.26) produces

$$
\nu_{t}=C_{\mu} k^{2} / \epsilon,
$$

for steady flow, with $C_{\mu}=0.11$. This is slightly higher than the value of $C_{\mu}=0.0 \mathrm{~S}$, established experimentally by Launder and Spalding (1974). 
Thus the single-relaxation-time approach produces, in part, the main features of the energy equation treatment and Reynolds stress used in the $k-\epsilon$ theory. An equation for the dissipation rate has not yet emerged from the method of Lundgren so the correspondence is still incomplete. Nevertheless, the foregoing discussion represents the beginnings of a fundamental theory.

\section{NONHOMOGENEOUS MOMENTUM EXCHANGE}

In Chapter III, Sec. 2 the discussion centered on the momentum exchange term. There the goal was to develop a low-order representation of the exchange force by introducing, in effect, a plausible form for the two-point probability distribution function $f_{1,2}^{(k, m)}$. This was accomplished by considering the various correlation coefficients related to $f_{1,2}^{(k, m)}$ in the case of a homogeneous statistical field.

The object of this section is to extend the Chapter III homogeneous result for the momentum exchange term to the nonhomogeneous case. This extension is approached in a manner that is consistent with the developments of Sec. 1. There the extension from homogeneous to nonhomogeneous was made for the Reynolds stress and other turbulence quantities. Here the main objective is io use ideas analogous to those employed in Sec. 1 to obtain a momentum exchange term that depends on mean flow gradients.

The momentum exchange force obtained in Chapter III, Sec. 2 is, for fluid $k$

$$
F_{i}^{(k)}=K\left\langle u_{i}^{(l-k)}\right\rangle
$$

where the scalar momentum exchange coefficient $K$ is

$$
K=[\rho]\left\langle\theta^{(1)}\right\rangle\left\langle\theta^{(2)}\right\rangle \frac{1}{s}\left(\beta_{1} \frac{[q]}{\left|\left\langle\mathbf{u}^{(21)}\right\rangle\right|}+\beta_{2} R e_{t}^{-1}+\frac{9}{2} R e_{v}^{-1}\right)\left|\left\langle\mathbf{u}^{(l k)}\right\rangle\right|,
$$

and has dimensions of (density)/(time). Equation (4.30) contains only a component of force parallel to the mean relative motion, that is, a drag component. A mean lift component can arise in the presence of velocity gradients (see Chapter I, Sec. 2). Here a modification to Eq. (4.30) will be considered that is designed to introduce the lift component of force in a way that depends on nonhomogeneities in the mean flow. The total force, possessing both lift and drag components, is referred to here as simply the friction force. In this context the friction force is the force per unit total volume acting on material $k$ as a result of a mean interpenetrating motion.

In general, any nonhomogeneities in the mean flow quantities can be responsible for altering the friction force from the homogeneous fcrm of Eq. (4.30). Spatial nonhomogeneities alter the viscous drag and form drag, and can be responsible for lift (Saffman force) and other forces. Temporal nonhomogeneities cause the added mass and Basset history effects (Maxey and Riley 1983). 
Consider now Eq. (4.30), modified by the addition of a nonhomogeneous term consisting of the gradient in the composite velocity

$$
\begin{aligned}
F_{i}^{(k)}=[\rho]\left\langle\theta^{(1)}\right\rangle\left\langle\theta^{(2)}\right\rangle & \left(\beta_{1} \frac{\mid q]}{\left|\left\langle\mathbf{u}^{(21)}\right\rangle\right|}+\beta_{2} R e_{t}^{-1}+\frac{9}{2} R e_{v}^{-1}\right) \\
& \cdot\left\{\frac{\left|\left\langle u^{(l-k)}\right\rangle\right|}{s} \delta_{i j}+\frac{\partial\left\langle u_{i}\right\rangle}{\partial x_{j}}\right\}\left\langle u_{j}^{(l-k)}\right\rangle,
\end{aligned}
$$

which reduces to (4.30) in the homogeneous case. A little reflection reveals that expression (4.32) contains a lift force for the simple case of a solid body moving in the direction of decreasing luid velocity in a pure shear flow.

The ideas behind Eq. (4.32) are the following. The momentum exchange coefficient $K$ can be thought of as the ratio of a characteristic density to a relaxation time. The inverse relaxation time in Eq. (4.31) is the magnitude of the mean relative velocity divided by the entity scale. The effect of Eq. (4.32) is to modify the (scalar) inverse relaxation time with another inverse time scale (a tensor) characteristic of velocity gradients in the mean flow. This tensor inverse time scale depends on both velocity and volume fraction gradients by way of the definition of the composite velocity

$$
\begin{aligned}
\langle\mathbf{u}\rangle & =\left\langle\theta^{(1)} \mathbf{u}+\theta^{(2)} \mathbf{u}\right\rangle \\
& =\left\langle\theta^{(1)}\right\rangle\left\langle\mathbf{u}^{(1)}\right\rangle+\left\langle\theta^{(2)}\right\rangle\left\langle\mathbf{u}^{(2)}\right\rangle .
\end{aligned}
$$

Also the combined relaxation time is by itself time-dependent because the mean relative velocity is in go .eral time-dependent.

The foregoing ideas reveal the speculative nature of Eq. (4.32), which stands for now as a simple postulate that remains to be tested by comparison with experimental data. Some of the necessary comparisons are presented in Chapter VI.

Another part of the momentum exchange term that was identified in Chapter IIT, Sec. 2, and also neglected there, is the thermal force. This part of the exchange force has not yet been specifically quantified for interpenetrating flows of the type considered here. For multispecies molecular flows, the thermal force is well known (Braginskii 1965). In that case, the thermal force occurs as a result of gradients in the mear molecular speed (temperature) of a given species of molecule, and can exist in the absence of a mean flow interpenetration of the various molecules. The physical reason for this is the following.

Consider a mixture of two molecule types, say molecules $a$ and $b$, both fluctuating about a zero mean velocity. Let the temperature (mean speed squared) of species $a$ be uniform in space, and suppose the temperature of species $b$ increases to the right. Now consider a plane perpendicular to the $b$ temperature gradient. The molecules of $b$ arriving at this plane from the right will have a larger average 
speed than the molecules of $b$ arriving from the left, by virtue of the $b$ temperature gradient. The net momentum lost by species $b$ in collisions with species $a$ results in an average force on $b$ to the right. This is because the rate of momentum loss by collision (collision frequency) is proportional to the difference in molecular speeds, so the $b$ molecules arriving from the right lose more momentum than those arriving from the left can gain, per unit of time (Braginskii 1965).

In the multimaterial case, the analogous effect can lead to a diffusion in mass. Such an effect has been considered in the literature, notably by Reeks (1983) who coins the term "turbophoresis" for this type of mass diffusion. The same effect is present in the present theory in the form of the thermal force. By analogy to the thermal force in a molecular mixture, the thermal force in a multiphase fow can be estimated for material $k$ by

$$
-[\rho]\left\langle\theta^{(1)}\right\rangle\left\langle\theta^{(2)}\right\rangle \frac{\partial}{\partial x_{i}}\left(q^{(l) 2}-q^{(k) 2}\right) .
$$

Here $q^{(k)}$ is again the root mean square velocity fluctuation for material $k$, and the factor of $\left\langle\theta^{(1)}\right\rangle\left\langle\theta^{(2)}\right\rangle$ is used to ensure that this part of the exchange force limits to zero if either material vanishes at a point. With this the total exchange furce on material $k$ can be written

$$
F_{i}^{(k)}=\{\rho]\left\langle\theta^{(k)}\right\rangle\left\langle\theta^{(l)}\right\rangle\left[K_{i j}^{\prime}\left\langle u_{j}^{(l-k)}\right\rangle-\frac{\partial}{\partial x_{i}}\left(q^{(l) 2}-q^{(k) 2}\right)\right]
$$

where

$$
\begin{aligned}
& K_{i j}^{\prime}=\left[\beta_{1} \frac{\mid q]}{\left|\left\langle\mathbf{u}^{(21)}\right\rangle\right|}+\beta_{2} R e_{t}^{-1}+\frac{9}{2} R e_{v}^{-1}\right] \\
& \cdot\left\{\frac{\left|\left\langle u^{(l k)}\right\rangle\right|}{s} \delta_{i j}+\frac{\partial\left\langle u_{i}\right\rangle}{\partial x_{j}}\right\}
\end{aligned}
$$

is the momentum exchange tensor for material $k$. In combining the friction and thermal exchange forces, the common factor $[\rho]\left\langle\theta^{(1)}\right\rangle\left\langle\theta^{(2)}\right\rangle$ is extracted. This can be thought of as the appropriate characteristic density for the momentum exchange rate. The term in brackets in Eq. (4.34) is the characteristic rate at which the velocity changes due to both average interpenetration and gradients in the fluctuational temperature of the two materials.

The exchange tensor $K_{i j}$ is an inverse time scale, and is expressed in the form of Eq. (4.35) in order to show its basic elements. Note that the term in square brackets is a nondimensional quantity composed of three parts. The first part represents the effect of form drag, the second represents the effect pre-existing turbulence in the fluid and the third represents the viscous shear effect at interfaces. The term in curly brackets in Eq. (4.35) is an inverse time scale (tensor) that 
has two parts. The isotropic part is based on the entity scale and the anisotropic part is the gradient in the composite velocity.

\section{References Cited}

Braginskii, S. I. 1965. Transport processes in a plasma. in M. A. Leontovich ed. Reviews of plasma physics, volume 1. New York: Consultants Bureau.

Launder, B. E. and D. B. Spalding. 1974. The numerical computation of turbulent flows. Comp. Meth. App. Mech. Eng. 3:269-289.

Lundgren, T. S. 1967. Distribution functions in the statistical theory of turbulence. Phys. Fluids. 10:969-975.

. 1969. Model equation for nonhomogeneous turbulence. Phys. Fluids. 12:485-497.

Maxey, M. R. and J. J. Riley. 1983. Equation of motion for a small rigid sphere in a nonuniform flow. Phys. Fluids. 26:883-889.

Reeks, M. W. 1983. The transport of discrete particles in inhomogeneous turbulence. J. Aerosol Sci. 14:729-739.

Yakhot, V. and S. A. Orszag. 1986. Renormalization group analysis of turbulence. I. Basic theory. J. Sci. Computing. 1:3-51. 


\section{Chapter $V$}

\section{Solution of the Average Equations}

The mean flow equations developed in the previous chapters can only be solved exactly for an extremely limited set of conditions. Most of the circumstances for which exact solutions can be obtained are of little interest in engineering or science. Fortunately, however, a great many problems exist for which approximate solutions are not only adequate but extremely useful.

In this chapter a numerical method for obtaining approximate solutions to the multimaterial mean flow equations is described. This method is a scheme developed for the iime-unsteady incompressible single-fluid flow equations. A single-fluid incompressible flow solution method is appropriate for the present multimaterial studies, because each material is considered to be incompressible. This enables expression of the mean pressure as a function of the other independent variables. To see this, consider an abbreviated set of general mean flow equations, neglecting turbulence and viscous effects. Consider, for example, the mean mass equation

$$
\frac{\partial \theta^{(k)}}{\partial t}+\frac{\partial}{\partial x_{j}} u_{j}^{(k)} \theta^{(k)}=0,
$$

the mean momentum equation

$$
\frac{\partial}{\partial t}\left(\theta^{(k)} u_{i}\right)+\frac{\partial}{\partial x_{j}} u_{j}^{(k)}\left(\theta^{(k)} u_{i}\right)=-\frac{\theta^{(k)}}{\rho^{(k)}} \frac{\partial p}{\partial x_{i}}+\theta^{(k)} g_{i}+\frac{1}{\rho^{(k)}} F_{i}^{(\mathfrak{k})}
$$

and the condition on the volume fractions

$$
\sum_{k=1}^{N} \theta^{(k)}=1
$$

where the angle brackets denoting averages have been dropped, and $N$ is the total numbers of materials. Notice that for $N=1$, these reduce to the equations for a single, nonviscous, nonturbulent, incompressible fluid. Summing Eq. (5.1) and using Eq. (5.3) yields the multimaterial incompressibility condition

$$
\sum_{k=1}^{N} \frac{\partial}{\partial x_{j}}\left(\theta^{(k)} u_{j}\right)=0
$$

where the definition of mean velocity 


$$
u_{j}^{(k)}=\frac{\left(\theta^{(k)} u_{j}\right)}{\theta^{(k)}}
$$

has been useci. The divergence of Eq. (5.2) can also be summed and the time derivative eliminated using (5.4). The result is

$$
\begin{aligned}
\frac{\partial^{2} p}{\partial x_{i}^{2}} \sum_{k=1}^{N} \frac{\theta^{(k)}}{\rho^{(k)}}+\frac{\partial p}{\partial x_{i}} \sum_{k=1}^{N} \frac{\partial}{\partial x_{i}}\left(\frac{\theta^{(k)}}{\rho^{(k)}}\right)= & -\sum_{k=1}^{N} \frac{\partial^{2}}{\partial x_{i} \partial x_{j}}-u_{j}^{(k)}\left(\theta^{(k)} u_{i}\right) \\
& +\sum_{k=1}^{N} \frac{1}{\rho^{(k)}} \frac{\partial}{\partial x_{i}} F_{i}^{(k)} .
\end{aligned}
$$

Equation (5.5) is a generalized Poisson equation, which reduces to

$$
\frac{\partial^{2} p}{\partial x_{i}^{2}}=-\rho \frac{\partial^{2} u_{i} u_{j}}{\partial x_{i} \partial x_{j}}
$$

in the single-fluid case.

The fact that Eq. (5.6) is linear in the pressure is frequently utilized in modern numerical methods for incompressible flow (Roache 1972). This may or may not be a significant feature of (5.5). The main point here is that the pressure can be expressed as a function of the other independent variables, allowing use of incompressible flow solution schemes in the case of $N>1$ (Amsden and Harlow 1978).

Equation (5.5) reveals the so-called elliptic nature of the system (5.1)-(5.3). This distinguishes the incompressible flow equations from the compressible flow equations, which are predominantly hyperbolic. In the latter case, the pressure is determined from an equation of state; an equation that reiates the pressure to, say, the density and internal energy per unit mass. Thus for compressible flow studies, one must retain a conservation equation for energy, in order to maintain a closed set of equations. (A notable exception to this is the case of the acoustic equations, which are utilized in the discussion of Sec. 3 on numerical accuracy.)

Sections 1-4 of this chapter present a new method for obtaining numerical solutions to Eqs. (5.1) $-(5.3)$ for the case $N=1$. The single-fluid case is selected so that the presentation is at first unencumbered with multimaterial details. The new method has, in part, already been described (Kashiwa 1986b). The discussion of Secs. 1-4 contains material that clarifies and extends the method already reported.

Section 5 is a discussion of how the full set of multimaterial mean flow equations from Chapter IV are solved, within the context of the procedure presented in Secs. 1-4. The final section is a description of the computer code constructed to carry out the numerical computations. There a variety of sample results are given to demonstrate the accuracy of the solutions obtained by the new method. 


\section{INTRODUCTION}

The numerical method described here belongs to the class of methods in computational fluid dynamics called "control volume" (CV) tecinniques. In a CV method, the flow domain is overlaid by a grid that subdivides the domain into an array of cells. Each cell is treated as an individual control volume, in which the conservation laws for mass, momentum, and energy are to be satisfied. CV techniques typically utilize the quantities mass, momentum, and total energy, all per unit volume, as the primary variables. (When the computational grid is allowed to move during the calculation, the volume of each cell can change, so the primary variables become the total amount of the foregoing quantities in each cell.) Hence the CV computation is physically based, in both its formulation and its result, making $\mathrm{CV}$ methods the most widely-used class of solution techniques in the field of computational fluid dynamics.

The method described here is distinguished from other CV methods for incompressible flow by its use of the same control volume for mass and linear momentum, rather than mass and noomentum control volumes that are staggered in space. Specification of coincident control volumes for mass and momentum obviates some rather restrictive difficulties associated with staggered-mesh schemes, and provides the advantages of single control volume methods that are commonly used for compressible flow problems.

The type of mesh staggering that is now widely used for incompressible flow calculations was invented by Harlow and Welch (1965), and was first introduc:ad in their Marker-and-Cell (MAC) method. The effective mesh staggering employed in the MAC method is to center momentum control volumes on the faces of mass control volumes. This gives rise to what appear as a cell-centered pressure and a cell-face normal velocity. This spatial location of the variables produces the coupling between the mass and momentum equations that is essential to the stability of the calculation.

In its original form, the MAC method is restricted to use on a computational mesh of rectangles. Recently, the MAC approach has been generalized to a socalled "finite-volume" method, whereby the conservation equations are solved in their integral form (Kashiwa 1986a). This enables the use of a nonorthogonal computational grid that is composed of arbitrary polygons.

There are some difficulties with the MAC staggered mesh scheme, either in the original or in the generalized form. First, the MAC method, in the original formulation, is restricted to use on a rectangular grid. This makes simulation in geometries with curved boundaries cumbersome, since a coordinate transformation from a curved physical space to a rectangular computational space is usually required. When the boundary shape evolves with the solution, this transformation becomes excessively complicated. The generalized (finite-volume) MAC technique (Kashiwa 1986a) does not require the coordinate transformation but the complexity of the general staggered grid is excessive, which offers a severe limitation to its 
use.

A second difficulty, which can be more restrictive than the first, involves the noncoincidence in spatial location of mass and momentum in the MAC formulation. For problems in which the density is a constant in space, this spatial staggering poses no difficulty. However, for problems in which the mass per unit total volume can vary, as in the case of incompressible multimaterial flow, the spatial staggering can become prohibitive. This is because the fluid velocity, defined as the ratio of momentum per unit total volume to mass per unit total volume, is a quantity to be derived from the primary variables. The calculation of the velocity is therefore inconsistent if the mass and momentum are conserved in different control volumes. An inconsistent velocity calculation leads to spurious resu!ts from the advective part of the calculation, particularly in multimaterial simulations. Whereas multimaterial simulations have been performed using a staggered mesh (Amsden and Harlow 1978), special care must be used in regions where the volume fraction of one material tends toward zero, in order to avcid the adverse effects of the spurious velocity calculation.

Another staggering scheme is one that has been used for compressible flow computations. This is the method introduced very early on by Von Neumann and Richtmyer (1950) in one-dimensional coordinates, and used in multiple dimensions by Hirt et al. (1974) in the Arbitrary-Langrangian-Eulerian (ALE) method. In the ALE finite-volume method, momentum control volumes are centered at the vertices of mass control volumes so the pressure appears cell-centered and the velocity appears vertex-centered. In addition to the problem of deriving a consistent velocity, the ALE mesh staggering suffers from an added difficulty. With the velocity at mass control volume vertices, certain spatially alternating disturbances in the velocity field do not result in a change in volume of the mass control volume, in multiple space dimensions. The result is the growth in these alternating disturbances and eventually the appearance of spurious patterns in the pressure field.

The various difficulties with $\mathrm{CV}$ computing methods using staggered control volumes are avoided when a coincident control volume is used for mass and momentum. The CV method of MacCormack (1969) for compressible flows is one of the best-known, and most successful, nonstaggered CV methods. More secently, the Godunov method has emerged as a successful single CV method for multidimerisional calculation of compressible flows (Woodward and Collela 1984). For fully incompressible flows, a successful single CV method has not yet been described.

The next three sections contain a description of a single control volume method for incompressible flow computation. The method makes use of the control volume ideas embodied in the explicit predictor-corrector scheme of MacCormack (1969) for compressible flow calculations. 


\section{BASIC PREDICTOR-CORRECTOR METHOD}

The essence of the method can be grasped by consideration of the onedimensional conservation laws for a single, nonviscous, incompressible fluid without gravity. The differential form of these equations is

$$
\begin{gathered}
\frac{\partial u}{\partial x}=0 \\
\frac{\partial u}{\partial t}+\frac{\partial u^{2}}{\partial x}=-\frac{1}{\rho} \frac{\partial p}{\partial x}
\end{gathered}
$$

Here $u$ and $p$ are the velocity and pressure respectively, which are the unknown functions of position $x$ and time $t$, and $\rho$ is the constant fluid density. The concern here is with how Eqs. (5.7) and (5.8) are approximated at logical location $j$ along the domain $0 \leq x \leq 1$, subdivided into $N$ intervals $\Delta x=1 / N$ in length, such that the physical position is $x_{j}=\left(j-\frac{1}{2}\right) \Delta x$, for $j=1,2, \ldots, N$. Initial and boundary conditions will be discussed in a later section. The numerical procedure for finding $u_{j}^{n+1}$ and $p_{j}^{n+1}$, the new time level unknowns, given knowledge of $u_{j}^{n}$ and $p_{j}^{n}$, the unknowns at time $n \Delta t$, is a two-step procedure. The predictor step at cell $j$ is defined by

$$
\begin{gathered}
0=\frac{1}{2 \Delta x}\left(u_{j+1}^{\overline{n+1}}-u_{j-1}^{\overline{n+1}}\right) \\
u_{j}^{\overline{n+1}}=u_{j}^{n}-\frac{\Delta t}{\Delta x} A\left(u_{j}^{n}\right)-\frac{\Delta t}{\rho \Delta x}\left(p_{j+1}^{\overline{n+1}}-p_{j}^{\overline{n+1}}\right),
\end{gathered}
$$

and the corrector step defined by

$$
\begin{gathered}
0=\frac{1}{2 \Delta x}\left(u_{j+1}^{n+1}-u_{j-1}^{n+1}\right) \\
u_{j}^{n+1}=\frac{1}{2}\left\{u_{j}^{n}+u_{j}^{\overline{n+1}}-\frac{\Delta t}{\Delta x} \mathcal{A}\left(u_{j}^{\overline{n+1}}\right)-\frac{\Delta t}{\rho \Delta x}\left(p_{j}^{n+1}-p_{j-1}^{n+1}\right)\right\} .
\end{gathered}
$$

In these, the superscript $\overline{n+1}$ denotes the predictor (provisional or auxiliary) quantities, and $A$ is an advection operator. (Because the advective part of the calculation does not represent the essential newness of the method, precise definition of $A$ will be momentarily postponed.) The main thing to observe about (5.9)-(5.12) is the type of differencing used. The mass equation in both predictor and corrector steps is approximated with central differences. This means that the finite-difference approximation to the mass equation is second-order accurate in space. The pressure gradient term in the predictor step is approximated with a forward difference. In the corrector step a backward difference is used in the pressure 
gradient term. This choice of spatial differencing in the pressure gradient term was introduced by MacCormack (1969) for compressible flow calculations. The effect of the two-step procedure is to make the net pressure acceleration second-order accurate in space. In Sec. 3 it will be seen that the procedure is also formally second-order accurate in time. ("Second-order" is used here in the popular sense that such accuracy is obtained on a uniform grid.) For the case of incompressible flows, the use of one-sided differences in the pressure gradient terms, in each of the predictor and corrector steps, assures the required coupling between the mass and momentum equations.

A second crucial feature of the approximation in Eqs. (5.9)-(5.12) is that each of the predictor and corrector steps are implicit in the independent variables. This implicitness is the key to enabling numerical solution of elliptic equation systems, for which the speed of signal propagation is effectively infinite (Harlow and Welch 1965). Solution of the implicit equation systems is facilitated by the fact that the Poisson equation for the pressure is linear in the pressure. The finitedifference approximation to (5.6) for the predictor step is obtaijed by placing Eq. (5.10) into Eq. (5.9). The result is

$$
\frac{1}{\Delta x^{2}}\left[p_{j+2}^{\overline{n+1}}-p_{j+1}^{\overline{n+1}}-p_{j}^{\overline{n+1}}+p_{j-1}^{\overline{n+1}}\right]=-\frac{\rho}{\Delta x^{2}}\left[\mathcal{A}\left(u_{j+1}^{n}\right)-\mathcal{A}\left(u_{j-1}^{n}\right)\right],
$$

which states that the predictor pressure satisifies a first-order (asymmetric) approximation to Eq. (5.6). Similarly the corrector pressure satisifes

$$
\frac{1}{\Delta x^{2}}\left[p_{j+1}^{n+1}-p_{j}^{n+1}-p_{j-1}^{n+1}+p_{j-2}^{n+1}\right]=-\frac{\rho}{\Delta x^{2}}\left[A\left(u_{j+1}^{\overline{n+1}}\right)-\mathcal{A}\left(u_{j-1}^{\overline{n+1}}\right)\right] .
$$

Clearly the predictor and corrector pressures can be quite different under time-unsteady flow conditions or in regions where the pressure gradient is nonlinear. This is because the right side of (5.13) involves $n$-time velocities and the right side of (5.14) $n+1$-time velocities, and because of the asymmetry of the left sides. The pressure that effectively produces the acceleration can be observed by combining Eqs. (5.10) and (5.12). With some rearrangement the result is

$$
\frac{1}{\Delta t}\left(u_{j}^{n+1}-u_{j}^{n}\right)+\frac{1}{2 \Delta x}\left[\mathcal{A}\left(u_{j}^{n}\right)+\mathcal{A}\left(u_{j}^{\overline{n+1}}\right)\right]=-\frac{1}{\rho \Delta x}\left[p_{j+1 / 2}^{*}-p_{j-1 / 2}^{*}\right],
$$

where

$$
p_{j+1 / 2}^{*}=\frac{1}{2}\left(p_{j+1}^{\overline{n+1}}+p_{j}^{n+1}\right)
$$

is the effective pressure. The half-index on the effective pressure $p^{*}$ is used here to emphasize the idea that $p^{*}$ is a quantity located at the edges of the computational 
cell. Thus a cell-edge pressure emerges as a natural consequence of the predictorcorrector formulation.

The advection operator that has proved very useful for the foregoing method is a modified form of the scheme introduced by van Leer (1977). This is

$$
\mathscr{A}\left(u_{j}\right)=\left[\begin{array}{ll}
\tilde{u}_{j+1 / 2} & u_{j+1 / 2}-\tilde{u}_{j-1 / 2} u_{j-1 / 2}
\end{array}\right],
$$

in which

$$
u_{j+1 / 2}=\frac{1}{2}\left(u_{j}+u_{j+1}\right)
$$

and

$$
\tilde{u}_{j+1 / 2}= \begin{cases}u_{j}+\frac{\Delta x}{2}\left(\left(\frac{\partial u}{\partial x}\right)\right)_{j}, & u_{j+1 / 2}>0 \\ u_{j+1}-\frac{\Delta x}{2}\left(\left(\frac{\partial u}{\partial x}\right)\right)_{j+1}, & u_{j+1 / 2}<0\end{cases}
$$

where

$$
\left(\left(\frac{\partial u}{\partial x}\right)\right)_{j}=\frac{1}{2 \Delta x}\left(u_{j+1}-u_{j-1}\right) .
$$

In this the time level is $n$ for the predictor step and $\overline{n+1}$ for the corrector step. This advection operator can be interpreted as follows. The velocity with which the material flux is formed is given by (5.17b). The quantity to be transported, in this case the horizontal momentum per unit mass, is given by $(5.17 \mathrm{c})$. The expression in $(5.17 \mathrm{c})$ defines the transported quantity at a cell face as the first two terms in a Taylor series expansion in space about the upstream cell center. The derivative in the Taylor series, Eq. (5.17d), is a second-order numerical estimate of the gradient in the quantity, also located at the upstream cell center. Notice that (5.17b) can be used to rewrite Eqs. (5.19) and (5.11)

$$
\begin{aligned}
& 0=\frac{1}{\Delta x}\left(u_{j+1 / 2}^{\overline{n+1}}-u_{j-1 / 2}^{\overline{n+1}}\right) \\
& 0=\frac{1}{\Delta x}\left(u_{j+1 / 2}^{n+1}-u_{j-1 / 2}^{n+1}\right)
\end{aligned}
$$

so the same fluxing velocity appears in both the mass equation and the advection operator (with the exception of the time level). This demonstrates a consistency in the treatment of the mass and momentum equations because the mass equation is essentially the advection operator, operating with the time advanced velocity, where the transported quantity is unity.

The description of the basic predictor-corrector method is now complete, with the exception of describing the way in which initial and boundary conditions are 
to be specified. These specifications are exceptionally simple for this method, and will be defined in Sec. 4 where the multidimensional implementation of the method is discussed. Before that however, some comments regarding accuracy and stability of the basic method must be made.

\section{ACCURACY AND STABILITY}

Understanding the degree to which the finite-difference equations (5.12)(5.15) are an accurate approximation to the differential equations (5.10) and (5.11) is an essential facet of numerical analysis. Another crucial part of this analysis is understanding the condition on the time increment $\Delta t$ that must be satisfied in order to ensure stable time integration of the equations. This section provides an insight into these two important parts of the numerical solution process.

A convenient way to gain an appreciation for the accuracy and stability properties of the foregoing method is to consider a linearized form of the compressible flow equations called the acoustic equations. In one space dimension the acoustic equations are (Vincenti and Kruger 1965)

$$
\begin{aligned}
& \frac{\partial \rho}{\partial t}+\rho_{0} \frac{\partial u}{\partial x}=0 \\
& \frac{\partial u}{\partial t}+\frac{1}{\rho_{0}} \frac{\partial p}{\partial x}=0 \\
& p=a^{2}\left(\rho-\rho_{0}\right)
\end{aligned}
$$

where the density $\rho$, velocity $u$ and pressure $p$ are functions of position $x$ and time $t$. Here $a^{2}$ is a constant coefficient related to the square of the signal propagation speed, and $\rho_{O}$ is a constant reference density. The state relation (5.22) can be used in (5.20) to obtain an alternate form of the mass equation

$$
\frac{1}{a^{2}} \frac{\partial p}{\partial t}+\rho_{0} \frac{\partial u}{\partial x}=0 \text {. }
$$

In the limit $a^{2} \rightarrow \infty$ Eq. (5.23) reduces to Eq. (5.7). Note that Eq. (5.21) is a linearized form of Eq. (5.8), with $\rho=\rho_{0}$. Thus for $a^{2} \rightarrow \infty$, the acoustic equations become the linearized incompressible flow equations in one dimension. Because (5.20)-(5.22) approach the incompressible flow equations smoothly with increasing $a^{2}$, one can expect that the accuracy and stability properties of the method, applied to the acoustic equations, will be relevant to the incompressible flow equations.

In the paragraphs that follow, Eqs. (5.20)-(5.22) are approximated by a succession of related predictor-corrector differencing formulas. This is done in order to provide an understanding of the basis for the scheme given by Eqs. (5.9)-(5.12). The analysis of the finite-difference formulas that is used here is based on the use 
of Taylor series expansions, and is known as truncation error analysis (Hirt, 1968). This simplified sort of analysis facilitates the display of both accuracy and stability properties of finite-difference expressions.

The first differencing scheme to be analyzed is the standard, explicit MacCormack (1969) method. This is shown in order to demonstrate the common fundamental properties of the scheme given in the previous section. The next step is to demonstrate the most obvious implicit generalization of MacCormack's method. This is shown to be defective in a way that is cured by use of the scheme given by Eqs. (5.9)-(5.12).

To begin, consider the well-known explicit MacCormack (1969) method applied to Eqs. (5.23) and (5.21). The predictor step is

$$
\begin{gathered}
p_{j}^{\overline{n+1}}=p_{j}^{n}-a_{2} \rho_{0} \frac{\Delta t}{\Delta x}\left(u_{j}^{n}-u_{j-1}^{n}\right), \\
u_{j}^{\overline{n+1}}=u_{j}^{n}-\frac{1}{\rho_{0}} \frac{\Delta t}{\Delta x}\left(p_{j}^{n}-p_{j-1}^{n}\right),
\end{gathered}
$$

and the corrector step is

$$
\begin{gathered}
p_{j}^{n+1}=p_{j}^{n}-\frac{a^{2} \rho_{0}}{2} \frac{\Delta t}{\Delta x}\left(u_{j}^{n}-u_{j-1}^{n}+u_{j+1}^{\overline{n+1}}-u_{j}^{\overline{n+1}}\right), \\
u_{j}^{n+1}=u_{j}^{n}-\frac{1}{2 \rho_{0}} \frac{\Delta t}{\Delta x}\left(p_{j}^{n}-p_{j-1}^{n}+p_{j+1}^{\overline{n+1}}-p_{j}^{\overline{n+1}}\right) .
\end{gathered}
$$

The important thing to note about the foregoing expressions is the use of backward differences in both the $n$-time velocity and pressure in the predictor step. The corrector step uses forward differences in the $\overline{n+1}$-time quantities. The effect of this procedure can be seen by combining, for example, Eqs. (5.26) and (5.24). The result can be written

$$
\frac{1}{\Delta t}\left(p_{j}^{n+1}-p_{j}^{n}\right)+\frac{a^{2} \rho_{0}}{2 \Delta x}\left(u_{j+1}^{n}-u_{j-1}^{n}\right)=\frac{a^{2} \Delta t}{2 \Delta x^{2}}\left(p_{j+1}^{n}-2 p_{j}^{n}+p_{j-1}^{n}\right)
$$

The accuracy of this expression is determined by expanding the variables spatially about point $j$, and temporally about time $n \Delta t$. The result of such an expansion is

$$
\frac{\partial p}{\partial t}+\frac{\Delta t}{2} \frac{\partial^{2} p}{\partial t^{2}}+a^{2} \rho_{0} \frac{\partial u}{\partial x}=\frac{a^{2} \Delta t}{2} \frac{\partial^{2} p}{\partial x^{2}}+O\left(\Delta t^{2}, \Delta x^{2}\right),
$$

where the symbol $O$ is used the standard fashion to represent "terms of order ...". Now observe that by differentiating Eq. (5.23) with respect to $t$ and subtracting Eq. (5.21), differentiated with respect to $x$, gives 


$$
\frac{\partial^{2} p}{\partial t^{2}}=a^{2} \frac{\partial^{2} p}{\partial x^{2}}
$$

so that in Eq. (5.28), the first term on the right exactly cancels the second term on the left, leaving the original differential equation plus only second-order truncation errors.

This result can be summarized as follows. The effect of the predictor-corrector formulation is to introduce a diffusive term on the right of Eq. (5.28) with the positive coefficient $\frac{1}{2} a^{2} \Delta t$. The forward-time difference term on the left of (5.28) brings in a term that, without compensation, would tend to introduce a destabilizing influence in the form of negative diffusion. The diffusive term on the right exactly cancels the destabilizing term, in a formally second-order fashion, giving the scheme the appearance of the so-called Lax-Wendroff (1964) method.

The condition on the time increment that must be satisfied in order to obtain a stable time integration using (5.24)-(5.27) is well known (MacCormack 1969). This condition, called the Courant-Freidricks-Lewy (CFL) condition, is

$$
\frac{|a+u| \Delta t}{\Delta x}<1
$$

which clearly forms an obstruction to using the explicit scheme when $a^{2} \rightarrow \infty$. This is the reason for development of implicit schemes, which are not restricted by the CFL condition.

As an intermediate step toward the implicit equations presented in the previous section, consider the implicit predictor step defined by

$$
\begin{aligned}
p_{j}^{\overline{n+1}} & =p_{j}^{n}-a^{2} \rho_{0} \frac{\Delta t}{\Delta x}\left(u_{j+1}^{\overline{n+1}}-u_{j}^{\overline{n+1}}\right), \\
u_{j}^{\overline{n+1}} & =u_{j}^{n}-\frac{1}{\rho_{0}} \frac{\Delta t}{\Delta x}\left(p_{j}^{\overline{n+1}}-p_{j-1}^{\overline{n+1}}\right)
\end{aligned}
$$

and the corrector step

$$
\begin{aligned}
& p_{j}^{n+1}=p_{j}^{n}-\frac{a^{2} \rho_{0}}{2} \frac{\Delta t}{\Delta x}\left(u_{j+1}^{\overline{n+1}}-u_{j}^{\overline{n+1}}+u_{j}^{n+1}-u_{j-1}^{n+1}\right), \\
& u_{j}^{n+1}=u_{j}^{n}-\frac{1}{2 \rho_{0}} \frac{\Delta t}{\Delta x}\left(p_{j}^{\overline{n+1}}-p_{j-1}^{\overline{n+1}}+p_{j+1}^{n+1}-p_{j}^{n+1}\right) .
\end{aligned}
$$

Notice that the predictor step uses a forward difference in the velocity and a backward difference in the pressure, both at $\overline{n+1}$-time. This has been referred to as using "oppositen differences (Kashiwa 1986b). The corrector step uses opposite differences in the $n+1$-time unknowns. There are two reasons for using opposite differences here. One reason is that a more accurate expression is obtained in 
doing so. The second and more important reason is that such a prescription is the only way that the boundary conditions can be satisfied in the incompressible limit.

The accuracy and stability properties of (5.31)-(5.34) can be observed by placing Eqs. (5.32) and (5.34) into (5.33). This produces

$$
\begin{aligned}
\frac{1}{\Delta t}\left(p_{j}^{n+1}-p_{j}^{n}\right)+\frac{a^{2} \rho_{0}}{2 \Delta x}\left(u_{j+1}^{n}-u_{j-1}^{n}\right)=\frac{a^{2} \Delta t}{2 \Delta x^{2}} & {\left[\left(p_{j+1}^{\overline{n+1}}-2 p_{j}^{\overline{n+1}}+p_{j-1}^{\overline{n+1}}\right)\right.} \\
& +\frac{1}{2}\left(p_{j+1}^{n+1}-2 p_{j}^{n+1}+p_{j-1}^{n+1}\right) \\
& \left.+\frac{1}{2}\left(p_{j+2}^{\overline{n+1}}-2 p_{j+1}^{\overline{n+1}}+p_{j}^{\overline{n+1}}\right)\right] .
\end{aligned}
$$

It can be seen by inspection of (5.35) that the destabilizing term from the forwardtime difference on the left is over-compensated by the diffusion term on the right. The diffusion term on the right is essentially a factor of two too large. Besides the asymmetry of the right side (note the last part of the diffusion term) which makes the scheme first-order accurate in space, the overcompensation has a more serious defect. A truncation error proportional to $\Delta t / \Delta x^{2}$ is present, producing a so-called "inconsistent" approximation (Roache 1972), which should always be avoided.

A second-order, consistent approximation can be obtained by replacing the on 乞-sided differences in Eqs. (5.31) and (5.33) with centered-difference expressions. This yields the basic implicit predictor-corrector scheme outlined in Sec. 2. For Eqs. (5.23) and (5.21) this produces the predictor step

$$
\begin{gathered}
{f_{j}^{\overline{n+1}}}^{\bar{n}}=p_{j}^{n}-a^{2} \rho_{0} \frac{\Delta t}{2 \Delta x}\left(u_{j+1}^{\overline{n+1}}-u_{j-1}^{\overline{n+1}}\right), \\
u_{j}^{\overline{n+1}}=u_{j}^{n}-\frac{1}{\rho_{0}} \frac{\Delta t}{\Delta x}\left(p_{j+1}^{\overline{n+1}}-p_{j}^{\overline{n+1}}\right),
\end{gathered}
$$

and the corrector step

$$
\begin{gathered}
p_{j}^{n+1}=p_{j}^{n}-\frac{a^{2} \rho_{0}}{2} \frac{\Delta t}{2 \Delta x}\left(u_{j+1}^{\overline{n+1}}-u_{j-1}^{\overline{n+1}}+u_{j-1}^{n+1}-u_{j+1}^{n+1}\right) \\
u_{j}^{n+1}=u_{j}^{n}-\frac{1}{2 \rho_{0}} \frac{\Delta t}{\Delta x}\left(p_{j+1}^{\overline{n+1}}-p_{j}^{\overline{n+1}}+p_{j}^{n+1}-p_{j-1}^{n+1}\right) .
\end{gathered}
$$

Combining Eqs. (5.37) and (5.39) into Eqs. (5.38) produces 


$$
\begin{aligned}
\frac{1}{\Delta t}\left(p_{j}^{n+1}-p_{j}^{n}\right)+\frac{a^{2} \rho}{2 \Delta x}( & \left.u_{j+1}^{n}-u_{j-1}^{n}\right) \\
=\frac{a^{2} \Delta t}{2 \Delta x^{2}}[ & {\left[\frac{3}{4}\left(p_{j+1}^{\overline{n+1}}-p_{j}^{\overline{n+1}}-p_{j-1}^{\overline{n+1}}+p_{j-2}^{\overline{n+1}}\right)\right.} \\
& \left.\quad+\frac{1}{4}\left(p_{j+2}^{n+1}-p_{j+1}^{n+1}-p_{j}^{n+1}+p_{j-1}^{n+1}\right)\right],(5.40)
\end{aligned}
$$

which is to be examined for accuracy and stability. The second-order accuracy property of $(5.40)$ can only be shown for a limited case. That is the case of timesteady flow, with at most a linear spatial variation in pressure. Then $p_{j}^{n+1}=$ $p_{j}^{n+1}$, and the right side of $(5.40)$ can be demonstrated to be a spatially secondorder accurate expression for $\frac{1}{2} a^{2} \Delta t \nabla^{2} p$ in the time-advanced pressure. The timeadvanced nature of the diffusion term endows the expression with unconditional stability so that the CFL condition is removed.

Under the general circumstances of time-unsteady flow with pressure gradients that are noniiiear, the accuracy and stability of (5.36)-(5.39) are difficult to prove analytically. Therefore the proof is approached experimentally by performing numerical calculations. In Sec. 6 of this chapter, a variety of calculations designed to demonstrate the performance of the numerical solution technique, are shown. So far, the experimenital result is that the implicit predictor-corrector method defined by Eqs. (5.9)-(5.12) is stable and accurate for all types of incompressible flov's.

\section{FINITE-VOLUME GENERALIZATION}

Up to now, the discussion of the numerical solution technique bas focused on the simplest form of the differential equations in one space dimension. Now that an impression of the numerical method has been established, the discussion can turn to a description of the scheme in multiple space dimensions. Before discussing the full set of multimaterial mean flow equations, however, the multidimensional solution of the laminar, viscous single-fluid conservation equations will be considered. This is done as an incremental step toward numerical solution of the average equations developed in Chapter IV. Hence this section displays the generalized form of the differencing scheme given in Sec. 2 , and discusses details of initial and boundary condition specifications. A variety of other more subtle facets of the solution procedure also discussed here.

A convenient way to obtain numerical solutions to the conservation equations in multiple dimensions is to discretize the integral form of the equations. Numerical procedures for solving the integral form of the conservation laws have been called "finite-volume" methods. The finite-difference equations presented in Secs. 2 and 3 of this chapter can be derived from the more general finite-volume 
equations for the special case of a uniform rectangular computational mesh. The main advantage of the finite-volume discretization of the integral form of the equations is that a computational mesh composed of arbitrarily shaped cells can be used. This facilitates the realistic representation of curved boundaries and irregular shaped computational domains, which could otherwise become quite difficult with a scheme limited to a rectangular mesh.

The mass and momentum conservation laws for an incompressible, viscous fluid can be written for a volume $V$ bounded by a surface $S$ with outward normal $\hat{\mathbf{n}}$

$$
\frac{1}{V} \int_{S} \mathbf{u} \cdot \hat{\mathbf{n}} d a=0
$$

and

$$
\begin{aligned}
\frac{d}{d t} \int_{V} \mathbf{u} d v+\int_{S} \mathbf{u} \mathbf{u} \cdot \hat{\mathbf{n}} d a= & -\int_{S}\left(\frac{p}{\rho}-\phi\right) \hat{\mathbf{n}} d a \\
& +\frac{1}{\rho} \int_{S} \vec{\tau} \cdot \hat{\mathbf{n}} d a
\end{aligned}
$$

where $\phi$ is the gravitational potential and the viscous stress tensor $\vec{\tau}$, for a Newtonian fluid, is

$$
\vec{\tau}=\mu\left[(\nabla \mathbf{u})+(\nabla \mathbf{u})^{T}\right],
$$

where $\mu$ is fluid viscosity. Notice that the gravitational acceleration has been expressed as the gradient of a potential, which is correspondingly converted to a surface integral and combined with the pressure acceleration term. If the acceleration due to gravity is considered to be a constant, the appropriate potential is

$$
\phi=\mathbf{g} \cdot \mathbf{r}
$$

where $\mathbf{g}$ is the constant acceleration and $\mathbf{r}$ is the position vector relative to an arbitrary but fixed point of reference. The reasons for expressing the gravitational term in this way will be discussed shortly.

The multidimensional discretization of Eqs. (5.41)-(5.42) that corresponds to Eqs. (5.9)-(5.12) is as follows. The predictor step for each cell in the mesh is defined

$$
\frac{1}{V} \sum_{\alpha} \frac{1}{2}\left(\mathbf{u}_{\alpha, b}^{\overline{n+1}}+\mathbf{u}_{\alpha, f}^{\overline{n+1}}\right) \cdot \mathbf{S}_{\alpha}=0
$$




$$
\mathbf{u}^{n+1}=\mathbf{u}^{n}-\frac{\Delta t}{V} \mathcal{A}\left(\mathbf{u}^{n}\right)-\frac{\Delta t}{V} \sum_{\alpha}\left(\frac{1}{\rho} p_{\alpha, f}^{\overline{n+1}}-\phi_{\alpha, f}\right) \mathbf{S}_{\alpha}+\frac{\Delta t}{\rho V} \sum_{\alpha} \vec{\tau}_{\alpha}^{n} \cdot \mathbf{S}_{\alpha},
$$

where the summations are over the sides of the computational cell with volume $V$, with outward-directed surface vecior $\mathbf{S}_{\alpha}$ ( $\hat{\mathbf{n}}$ times the cell-side area), and $A$ is again the advection operator. The subscripts $(\alpha, b)$ and $(\alpha, f)$ refer respectively to the cell-centered quantities on the $b$. - kward and forward sides of cell face $\alpha$ in logical space. The viscous stress is a cell-side quantity given by

$$
\vec{\tau}_{\alpha}^{n}=\mu\left[\llbracket \nabla \mathbf{u}^{n} \rrbracket_{\alpha}+\llbracket \nabla \mathbf{u}^{n} \rrbracket_{\alpha}^{T}\right],
$$

where $\llbracket \nabla \mathbf{u}^{n} \rrbracket_{\alpha}$ is a cell-side numerical estimate of the gradient in the velocity at $c \in l l$ face $\alpha$. Appendix A discribes a convenient way to obtain accurate numerical estimates of gradients. The gravitational potential is given directly by Eq. (5.44), where the position vector involved is that of the cell-center.

The corrector step at each cell of the mesh is defined

$$
\begin{gathered}
\frac{1}{V} \sum_{\alpha} \frac{1}{2}\left(\mathbf{u}_{\alpha, b}^{n+1}+\mathbf{u}_{\alpha, f}^{n+1}\right) \cdot \mathbf{S}_{\alpha}=0 \\
\mathbf{u}^{n+1}=\frac{1}{2}\left\{\mathbf{u}^{n}+\mathbf{u}^{n+1}-\frac{\Delta t}{V} \mathcal{A}\left(u^{\overline{n+1}}\right)-\frac{\Delta t}{V} \sum_{\alpha}\left(\frac{1}{\rho} p_{\alpha, b}^{n+1}-\phi_{\alpha, b}\right) \mathbf{S}_{\alpha}\right. \\
\left.+\frac{\Delta t}{\rho V} \sum_{\alpha} \overrightarrow{\vec{\tau}}_{\alpha}^{\overline{n+1}} \cdot \mathbf{S}_{\alpha}\right\}
\end{gathered}
$$

in which $\vec{\tau}_{\alpha}^{\overline{n+1}}$ is given by Eq. (5.46) with the $\overline{n+1}$-time velocity replacing the $n$-time velocity.

To clarify the meaning of "backward" and "forward" in logical space, corsider the typical cell drawn in two-dimensional logical coordinates shown in Fig. $r$. Figure " 7 shows a typical cell in a logically square mesh, which is a widely used arrangement because of the corresponding convenience provided by the array data storage system used in modern computers. In three space dimensions the foregoing computational cell becomes a cube. In Fig. 1a, cell-center $b$ is on the logical forward side of cell-side 1. Cell-center $a$ is on the logical backward side of cellside 1, which is also the logical backward side of cell side 2, and so on. Notice that Eq. (5.45) for the predictor step uses the forward-side pressure and gravitational potential. Equation (5.48) for the corrector step uses the correspoiiding data from the backward-side in logical space. This procedure gives rise to the forwardbackward finite-difference formulation described in Sec. 2. 

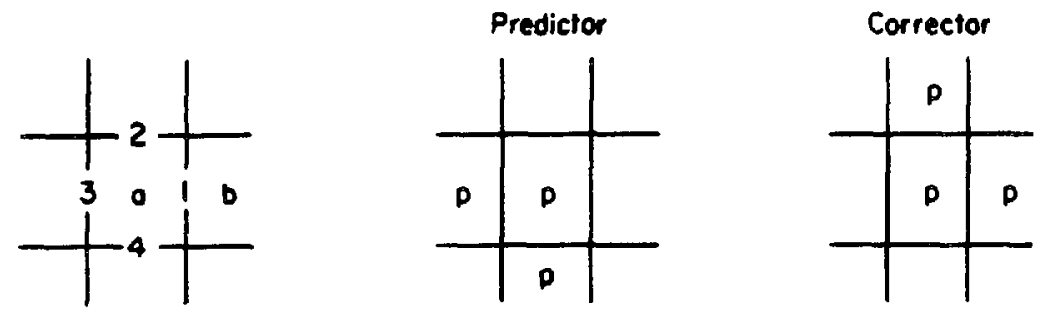

Figure 7. A typical computational cell in two-dimensional logical space.

Figure 7 provides a picture of the logical locations from which the pressure data is used in each of the predictor and corrector steps. In practice, the user should alternate between forward-backward and backward-forward differencing in the predictor-corrector scheme, in order to prevent a possible bias in the solution. (In two-dimensions there are four possible forward-backward differencing combinations among the coordinate directions. In three dimensions there are eight possible combinations. The user of schemes of this type should take care to rotate among all possible combinations in successive time-steps.)

Equations (5.44) and (5.45) represent a linear implicit system of equations for $\mathbf{u}^{\overline{n+1}}$ and $p^{n+1}$ in each cell of the mesh. As shown in Sec. 2, these two equations can be combined to form a Poisson equation for $p^{\overline{n+1}}$. The solution can be obtained by a variety of techniques from linear algebra. In this study, the simple relaxation procedure described in Appendix B has b/sen employed. Once the provisional quantities $u^{\overline{n+1}}$ and $\tilde{q}^{\overline{n+1}}$ are known, then Eqs. (5.47) and (5.48) define a second linear system in $\mathbf{u}^{n+1}$ and $p^{n+1}$. Thus the predictor-corrector procedure requires solving a meshwide, linear algebraic system of equations twice during each time increment.

The generalized form of the advection operator is

$$
A(\mathbf{u})=\sum_{\alpha} \tilde{\mathbf{u}}_{\alpha} v_{\alpha}
$$

in which the fluxing rate is

$$
v_{\alpha}=\frac{1}{2}\left(\mathbf{u}_{\alpha, b}+\mathbf{u}_{\alpha, f}\right) \cdot \mathbf{S}_{\alpha}
$$

and the momentum per unit mass fluxed at cell-side $\alpha$ is 


$$
\tilde{\mathbf{u}}_{\alpha}= \begin{cases}\mathbf{u}_{\alpha, b}+\left(\mathbf{x}_{\alpha}-\mathbf{r}_{\alpha, b}\right) \cdot((\nabla \mathbf{u}))_{\alpha, b}, & v_{\alpha}>0 \\ \mathbf{u}_{\alpha, \mathbf{f}}+\left(\mathbf{x}_{\alpha}-\mathbf{r}_{\alpha, f}\right) \cdot((\nabla \mathbf{u}))_{\alpha, f}, & v_{\alpha}<0\end{cases}
$$

where the velocity time level is $n$ for the predictor step and $\overline{n+1}$ for the corrector step, and $\mathbf{x}$ is the average position of cell-face $\alpha$. The quantity $v_{\alpha}$ can be thought of as the volume per unit time swept out by fluid passing cell-face $\alpha$. This same quantity is summed over the faces of the cell in Eqs. (5.44) and (5.47), at the advanced-time level, in calculation of the divergence in the velocity. Thus the rate at which mass and momentum are "fluxed" at the control volume surface (cell-edges) is identical (except for the time level). In (5.49c) the tensor $((\nabla u))$ is a numerical estimate of the cell-centered gradient in the velocity. Calculation of $((\nabla \mathbf{u}))$ is detailed in Appendix A.

Description of the finite-volume generalization of the basic predictor-corrector scheme is now only partly complete. There are four remaining subjects yet to be discussed. These are initial conditions, boundary conditions, the reasons for expressing the gravitational acceleration as the gradient in a potential, and specification of a stable time-step $\Delta t$.

Initialization of the independent variables is particularly simple in the foregoing numerical technique. This is because the initial velocity and pressure are completely arbitrary. That is to say, if an initial velocity and pressure are known, the values can be used to initialize the fields. If not, then any velocity and pressure can be inserted, and the calculation will produce an appropriate initial condition in the first time-step. To see this consider, for example, a horizontal flow in a uniform pipe with steady inflow at the left. Suppose the velocity and pressure are both specified initially to be zero. Equation (5.41) requires a solenoidal (divergence free or incompressible) velocity field, so in the first time increment the stationary fluid is accelerated impulsively to a solenoidal field resembling a potential flow. This flow will be uniform and equal to the specified velocity at the left. The pressure field in the first time-step corresponds to the pressure needed to accelerate the initially stationary fluid to the potential flow field. In successive time-steps, the velocity profile in the pipe develops toward the steady-flow solution, in a manner that duplicates the true dynamical behavior of such an impulsively started flow.

Boundary conditions on the velocity and pressure are also particularly simple in the present method. These can be summarized by a set of very simple "rules". The rule on the pressure is the following.

When the normal component of velocity at a boundary is specified, the pressure at the boundary is equal to the pressure at the adjacent cell center. When the velocity gradient normal to the boundary is specified, the pressure must be given a fixed value. 
The first part of the boundary pressure rule corresponds to a zero pressure gradient approximation at boundaries. This is consistent with the boundary-layer approximation on the pressure gradient, and is found to give quite accurate results (Kashiwa 1986b). The rule on velocity specification at boundaries is also simple. This is

Each of both the normal and tangential components of velocity can either have specified values or have a specified gradient. The usual specified gradient is zero, which means that the appropriate component of the adjacent cell-centered velocity is placed at the boundary.

This boundary condition rule contains within it a variety of boundary types. For example, if the normal component of velocity is set to zero, and the gradient in the tangential component is zero, then the boundary becomes a so-called freeslip wall (or equivalently a plane of symmetry, if the wall is flat). If instead the tangentia! component is set to zero the boundary becomes a no-slip stationary wall. Obviously the tangential component can be given a fixed value, making the boundary a no-slip moving wall.

There are two important types of "permeable" boundaries that $c \cdots:$ when a nonzero normal component of velocity is specified. The first is an inflow boundary, which arises when the normal component is given a value causing flow into the computational domain. (An inflow boundary can either be free-slip or no-slip.) The second type of permeable boundary occurs when the flow is predominantly outward from the computational domain. This outflow boundary can be a bit tricky and deserves a few special comments.

An outflow boundary is one for which the normal component is defined to be always outward from the computational domain. This means that if a flow field arises near the boundary causing the tendency to produce inflow at the outflow boundary, the inflow is cut off. (Consider a strong whirlpool approaching the exit of a channel.) Hence the interior flow can be affected by the outflow boundary in a nonphysical way. This is a basic and well-known difficulty with incompressible flow computation (Hirt et al. 1975). The way in which the difficulty can be avoided is to configure the computational mesh in such a way that a physically true outflow always occurs. This can usually be done by placing the outflow boundary far away from obstacles in the flow that may cause flow separation and subsequent development of large-scale fluid structures in the flow. Another way to limit the influence of an outflow boundary is to use instead zero normal velocity gradient specification, and fixed boundary pressures at the infiow and outflow boundaries.

The last comment in regard to the outflow boundary concerns the rate of convergence of the solution for the pressure. This convergence rate can be improved substantially if the total outtlow is continually normalized to equal the total inflow, throughout the process of solving the linear equation system. This assures that the net outflow always equals the net inflow so that the divergence condition on the velocity is satisfied globally at all times. For the relaxation procedure de- 
scribed in Appendix B, the foregoing practice can have a significant effect on the rate of convergence.

The rule for specification of the pressure at boundaries reveals the reason for using the gravitational potential in the gravity term. In a stationary hydrostatic pressure field, the pressure gradient exactly balances the acceleration due to gravity. If a velocity change like $g \Delta t$ is used, then the predictor-corrector scheme computes the appropriate pressure field, everywhere except at boundaries. Notice, for example, that at a left-hand zero gradient boundary, no pressure acceleration occurs when a backward pressure difference is used. The acceleration only occurs at the left end of the problem when a forward pressure difference is used, cwing to the zero pressure gradient specification at the boundary. The cell-centered gravitational potential provides a simple and handy way to perform the gravitational acceleration in a fashion that is consistent with the pressure acceleration. The following rule on the gravitational potential at boundaries assures this consistency.

When a zero pressure gradient is specified at a boundary, so must a zero gradient in the gravitational potential be applied. When a specified pressure condition is used, then the gravitational potential corresponding to the physical location of the boundary should be specified.

The foregoing completes the present discussion, with the exception of the time-step calculation. For this there are three conditions that must be satisfied in order to ensure a stable time integration. In one-dimensional form these are the explicit advection time-step condition

$$
\frac{|u| \Delta t}{\Delta x}<1,
$$

the explicit viscous diffusion condition

$$
\frac{\nu \Delta t}{\Delta x^{2}}<\frac{1}{2},
$$

and the explicit gravitational acceleration condition

$$
\Delta t \sqrt{g / \Delta x}<1,
$$

where $\nu=\mu / \rho$. The condition (5.50) arises because of the explicit advection operator. It states, in effect, that the net flux of material from a cell, in a single time-step, must be less than the total amount of material available in the cell. Condition (5.51) essentially states that the change in momentum by viscous diffusion, in a time $\Delta t$, must be less than the total momentum in the cell. Similarly, (5.52) requires that the gravitational acceleration must not cause the cell to become devoid of material, in a time $\Delta t$. This interpretation of the conditions (5.50)-(5.52) leads to the combined condition used in the finite-volume generalization 


$$
\frac{\Delta t}{2}\left\{\frac{1}{V} \sum_{\alpha}\left|v_{\alpha}\right|+\frac{2 \nu}{V^{2}} \sum_{\alpha} \mathbf{S}_{\alpha}^{2}+\sqrt{\frac{1}{V} \sum_{\alpha}\left|\mathrm{g} \cdot \mathrm{S}_{\alpha}\right|}\right\}<1,
$$

where $v_{\alpha}$ is the fluxing rate given by Eq. $(5.49 \mathrm{~b})$, and factor of $\frac{1}{2}$ is incorporated so that the expression reduces to a combination of $(5.50)-(5.52)$ in the onedimensional case. (Note that the length scale for the parts of (5.53) corresponding to (5.51) and (5.52) is the ratio of volume to total surface area for the cell.)

The discussion of the finite-volume form of the predictor-corrector solution method is now complete. The next step is to describe the use of the scheme for multimaterial problems, which is the subject of the next section.

\section{MULTIMATERIAL IMPLEMENTATION}

The purpose of this section to define in detail the way in which the full set of multimaterial mean flow equations are solved numerically in the applications presented in the next chapter. First the integral form of the equations is given. Then a brief description of the solution procedure is provided in words, followed by a complete listing of the discretized finite-volume equations.

For a volume $V$, fixed in space, the mass conservation equation for material $k=1,2$ is

$$
\frac{d}{d t}\left\langle\theta^{(k)}\right\rangle+\frac{1}{V} \int_{S}\left\langle\theta^{(k)}\right\rangle\left\langle\mathbf{u}^{(k)}\right\rangle \cdot \hat{\mathbf{n}} d a=0
$$

and the momentum conservation equation is

$$
\begin{aligned}
\frac{d}{d t}\left\langle\theta^{(k)} \mathbf{u}\right\rangle+\frac{1}{V} \int_{S}\left\langle\theta^{(k)} \mathbf{u}\right\rangle\left\langle\mathbf{u}^{(k)}\right\rangle \cdot \hat{\mathbf{n}} d a= & -\frac{\left\langle\theta^{(k)}\right\rangle}{V} \int_{S}\left(\frac{\langle p\rangle}{\rho^{(k)}}-\phi\right) \hat{\mathbf{n}} d a \\
& +\frac{1}{V} \int_{S} \nu^{(k)}\left\langle\theta^{(k)}\right\rangle \vec{D}^{(k)} \cdot \hat{\mathbf{n}} d a \\
& +\frac{1}{V} \int_{S} \overrightarrow{\vec{R}}^{(k)} \cdot \hat{\mathbf{n}} d a+\frac{1}{\rho^{(k)}} \mathbf{F}^{(k)}(5
\end{aligned}
$$

in which a typic..i element of the mean deformation rate tensor $\vec{D}^{(k)}$ is

$$
D_{i j}^{(k)}=\frac{\partial}{\partial x_{j}}\left\langle u_{i}^{(k)}\right\rangle+\frac{\partial}{\partial x_{i}}\left\langle u_{j}^{(k)}\right\rangle
$$

and a typical element of the Reynolds stress tensor $\vec{R}^{(k)}$ is 


$$
R_{i j}^{(k)}=\nu_{t}^{(k)}\left\langle\theta^{(\xi)}\right\rangle\left[D_{i j}^{(k)}-\frac{2}{3} \frac{\partial}{\partial x_{k}}\left\langle u_{k}^{(k)}\right\rangle \dot{\delta}_{i j}\right] .
$$

where the main diagonal part is assumed to be part of the mean pressure. Here the turbulent viscosity $\nu_{t}^{(k)}$ is

$$
\nu_{t}^{(k)}=C_{\mu} \frac{\left\langle k^{(k)}\right\rangle^{2}}{\left\langle\theta^{(k)}\right\rangle\left\langle\epsilon^{(k)}\right\rangle}
$$

The force per unit volume $\mathrm{F}^{(k)}$ for $l=2,1$ is given by

$$
F_{i}^{(k)}=[\rho]\left\{\theta^{(l)}\right\rangle\left\langle\theta^{(k)}\right\rangle\left[K_{i j}^{\prime}\left\langle u_{j}^{(l k)}\right\rangle-\frac{\partial}{\partial x_{i}}\left(q^{(l) 2}-q^{(k) 2}\right)\right]
$$

in which the momentum exchange function is

$$
K_{i j}^{\prime}=\left(\beta_{1} \frac{[q]}{\left|\left\langle\mathbf{u}^{(21)}\right\rangle\right|}+\beta_{2} R e_{t}^{-1}+\frac{9}{2} R e_{t}^{-1}\right)\left[\frac{\left|\left\langle\mathbf{u}^{(l k)}\right\rangle\right|}{s} \delta_{i j}+\frac{\partial\left\langle u_{i}\right\rangle}{\partial x_{j}}\right],
$$

with inverse viscous Reynolds number

$$
R e_{v}^{-1}=\frac{[\nu]}{\left|\left\langle\mathbf{u}^{(l k)}\right\rangle\right| s},
$$

and inverse turbulent Reynolds number

$$
\operatorname{Re}_{t}^{-1}=\frac{\left[\nu_{t}\right]}{\left|\left\langle\mathbf{u}^{(l k)}\right\rangle\right| s} .
$$

In Eq. (5.58) the mean turbulence energy per unit mass $\left\langle k^{(k)}\right\rangle$ satisfies

$$
\begin{aligned}
\frac{d}{d t}\left\langle k^{(k)}\right\rangle & +\frac{1}{V} \int_{S}\left\langle k^{(k)}\right\rangle\left\langle\mathbf{u}^{(k)}\right\rangle \cdot \hat{\mathbf{n}} d a=\frac{1}{V} \int_{S} \frac{\nu_{t}^{(k)}}{\sigma_{k}} \nabla\left\langle k^{(k)}\right\rangle \cdot \hat{\mathbf{n}} d a \\
& \left.+\left[P^{(k)}-\left\langle\epsilon^{(k)}\right\rangle+\frac{a^{(k)}}{\rho^{(k)}} F_{i}^{(k)}\left\langle u_{i}^{(k)}\right\rangle\right]\right]
\end{aligned}
$$

and the mean turbulence energy decay rate per unit mass $\left\langle\epsilon^{(k)}\right\rangle$ satisfies

$$
\begin{aligned}
\frac{d}{d t}\left\langle\epsilon^{(k)}\right\rangle & +\frac{1}{V} \int_{S}\left\langle\epsilon^{(k)}\right\rangle\left\langle\mathbf{u}^{(k)}\right\rangle \cdot \hat{\mathbf{n}} d a=\frac{1}{V} \int_{S} \frac{\nu_{t}^{(k)}}{\sigma_{\epsilon}} \nabla\left\langle\epsilon^{(k)}\right\rangle \cdot \hat{\mathbf{n}} d a \\
& +2\left\langle\theta^{(k)}\right\rangle[\nu] \nu^{(k)}\left(\frac{1}{V} \int_{S} \nabla\left\langle\mathbf{u}^{(k)}\right\rangle \cdot \hat{\mathbf{n}} d a\right)^{2} \\
& +\frac{\left\langle\epsilon^{(k)}\right\rangle}{\left\langle k^{(k)}\right\rangle}\left[C_{1} P^{(k)}-C_{2}\left\langle\epsilon^{(k)}\right\rangle\right]+C_{3} C_{\mu} \frac{a^{(k) 3 / 2}}{\rho^{(k)}} \frac{\left|\left\langle\mathbf{u}^{(21)}\right\rangle\right|}{s} F_{i}^{(k)}\left\langle u_{i}^{(l k)}\right\rangle
\end{aligned}
$$


where $P^{(k)}$ is the mean flow production rate

$$
P^{(k)}=R_{i j}^{(k)} \frac{\partial}{\partial x_{j}}\left\langle u_{i}^{(k)}\right\rangle
$$

the composite kinematic viscosity $[\nu]$ is

$$
\left\langle\theta^{(1)}\right\rangle \nu^{(1)}+\left\langle\theta^{(2)}\right\rangle \nu^{(2)}
$$

and the mean square velocity fluctuation is

$$
q^{(k) 2}=\frac{2}{3} \frac{\left\langle k^{(k)}\right\rangle}{\left\langle\theta^{(k)}\right\rangle} .
$$

The final equation, apart from defining a variety of derived quantities, is

$$
\sum_{k=1}^{2}\left\langle\theta^{(k)}\right\rangle=1 .
$$

For completeness the derived quantities are the mean velocity

$$
\left\langle\mathbf{u}^{(k)}\right\rangle=\frac{\left\langle\theta^{(k)} \mathbf{u}\right\rangle}{\left\langle\theta^{(k)}\right\rangle} .
$$

and the composite velocity

$$
\langle\mathbf{u}\rangle=\left\langle\theta^{(l)} \mathbf{u}\right\rangle+\left\langle\theta^{(k)} \mathbf{u}\right\rangle .
$$

The numerical solution of Eqs. (5.54)-(5.70) is characterized as follows. The mean mass and momentum conservation equations, which are the items of primary concern, are discretized according to a procedure corresponding to the one outlined in the previous section. The main extension required for the multimaterial case is the condition (5.68), which with (5.54) and (5.55) permits expression of the pressure as a function of the velocity. Thus the same basic solution method for the predictor and corrector implicit equation systems is used. The second term on the right side of (5.55) is the viscous stress term due to molecular viscosity. It is the same as the corresponding term in the single-fluid case, with the exception of a factor $\left\langle\theta^{(k)}\right\rangle$ inside the surface integral. Recall that the viscous stress is a cell-side quantity, and the volume fraction is cell-centered. This discrepancy in position is nicely handled within the predictor-corrector formulation by a forward-backward treatment of the volume fraction in this term. In the solution method used here, the viscous stress term is discretized in an explicit fashion, as it was in the singlefluid case already discussed. The third term on the right of (5.55) is the Reynolds stress term, which is also discretized explicitly. Notice that the turbulent viscosity, defined by (5.58), is a fundamentally cell-centered quantity, so that a forwardbackward treatment is required for accurate simulation of its effects. The last 
term in (5.55) is the exchange force, part of which is discretized implicitly, and part of which is handled explicitly. The nonhomogeneous parts of (5.59) are both calculated explicitly. The homogeneous part of the force is computed implicitly. This mixture of time-differencing provides an essential stability property to the calculation. That is, if the magnitude of $K_{i j}$ is large, a small relative mean velocity causes a very large acceleration. By placing the main part of the exchange force at the time-advanced level, time-step limitation due to momentum exchange is avoided.

For simplicity and ease of implementation Eqs. (5.63) and (5.64) for $\left\langle k^{(k)}\right\rangle$ and $\left\langle\epsilon^{(k)}\right\rangle$ are discretized explicitly. This means that special care must be exercised in selection of the time increment to ensure stability of the integration for these two equations. (In some cases the turbulence production and decay rates may become large, making this explicit treatment impractical. In this event the user could profit from expressing (5.63) and (6.64) as a point-wise implicit system of equations, whereby some selected parts of the right sides are placed at the timeadvanced level.)

Now the discretized form of (5.54)-(5.70) can be displayed. For the predictor step in a typical cell of the mesh, the mass equation is

$$
\left\langle\theta^{(k)}\right\rangle^{\overline{n+1}}=\left\langle\theta^{(k)}\right\rangle^{n}-\frac{\Delta t}{V} A\left(\left\langle\theta^{(k)}\right\rangle^{\overline{n+1}}\right),
$$

the condition $(5.68)$ is

$$
\left\langle\theta^{(k)}\right\rangle^{\overline{n+1}}+\left\langle\theta^{(l)}\right\rangle^{\overline{n+1}}=1,
$$

the momentum equation is

$$
\begin{aligned}
\left\langle\theta^{(k)} \mathbf{u}\right\rangle^{\overline{n+1}}=\left\langle\theta^{(k)} \mathbf{u}\right\rangle^{n} & -\frac{\Delta t}{V} A\left(\left\langle\theta^{(k)} \mathbf{u}\right\rangle^{n}\right) \\
& -\frac{\Delta t\left\langle\theta^{(k)}\right\rangle^{\overline{n+1}}}{V} \sum_{\alpha}\left(\frac{\dot{2}}{\rho^{(k)}}\langle p\rangle_{\alpha, f}^{\overline{n+1}}-\phi_{\alpha, f}\right) \mathbf{S}_{\alpha} \\
& +\frac{\Delta t \nu^{(k)}}{V} \sum_{\alpha}\left\langle\theta^{(k)}\right\rangle_{\alpha, f}^{n} \vec{D}_{\alpha}^{(k) n} \cdot \mathbf{S}_{\alpha} \\
& +\frac{\Delta t}{V} \sum_{\alpha} \vec{R}_{\alpha, f}^{(k) n} \cdot \mathbf{S}_{\alpha}+\frac{1}{\rho^{(k)}} \mathbf{F}^{(k) \overline{n+1}}
\end{aligned}
$$

the enery equation is 


$$
\begin{aligned}
\left\langle k^{(k)}\right\rangle^{\overline{n+1}} & =\left\langle k^{(k)}\right\rangle^{n}-\frac{\Delta t}{V} A\left(\left\langle k^{(k)}\right\rangle^{n}\right)+\frac{\Delta t}{V \sigma_{k}} B_{p}\left(\left\langle k^{(k)}\right\rangle^{n}\right) \\
& +\Delta t\left[P^{(k)}-\left\langle\epsilon^{(k)}\right\rangle+\frac{a^{(k)}}{\rho^{(k)}} F_{i}^{(k)}\left\langle u_{i}^{(l k)}\right\rangle\right]^{n},
\end{aligned}
$$

and the equation for the dissipation rate is

$$
\begin{aligned}
& \left\langle\epsilon^{(k)}\right\rangle^{\overline{i z+1}}=\left\langle\epsilon^{(k)}\right\rangle^{n}-\frac{\Delta t}{V} A\left(\left\langle\epsilon^{(\dot{k})}\right\rangle^{n}\right)+\frac{\Delta t}{V \sigma_{k}} B_{p}\left(\left\langle\epsilon^{(k)}\right\rangle^{n}\right) \\
& +\Delta t\left[\frac{\left\langle\epsilon^{(k)}\right\rangle}{\left\langle k^{(k)}\right\rangle}\left[C_{1} P^{(k)}-C_{2}\left\langle\epsilon^{(k)}\right\rangle\right]+C_{3} C_{\mu} \frac{a^{(k) 3 / 2}}{\rho^{(k)}} \frac{\left|\left\langle\mathbf{u}^{(21)}\right\rangle\right|}{s} F_{i}^{(k)}\left\langle u_{i}^{(l k)}\right\rangle\right]^{n} \\
& +\Delta t\left[2\left\langle\theta^{(k)}\right\rangle[\nu] \nu^{(k)}\left(\frac{1}{V} \sum_{\alpha}\left[\nabla\left\langle\mathbf{u}^{(k)}\right\rangle\right] \cdot \mathbf{S}_{\alpha}\right)^{2}\right]^{n}
\end{aligned}
$$

where $A$ is the advection operator and $B_{p}$ is the predictor diffusion operator. Also the net production terms in (5.74) and (5.75) are written in the form [ ${ }^{n}$, which means that everything inside the brackets is at time level $n$. The advection operator is defined

$$
A(Q)=\sum_{\alpha} \tilde{Q}_{\alpha} v_{\alpha}
$$

where the fluxing rate is

$$
v_{\alpha}=\frac{1}{2}\left[\mathbf{u}_{\alpha, b}+\mathbf{u}_{\alpha, f}\right] \cdot \mathbf{S}_{\alpha}
$$

and the fluxed quantity is

$$
\tilde{Q}_{\alpha}= \begin{cases}Q_{\alpha, b}+\left(\mathbf{x}_{\alpha}-\mathbf{r}_{\alpha, b}\right) \cdot((\nabla Q))_{\alpha, b}, & v_{\alpha}>0 \\ Q_{\alpha, f}+\left(\mathbf{x}_{\alpha}-\mathbf{r}_{\alpha, f}\right) \cdot((\nabla Q))_{\alpha, f}, & v_{\alpha}<0\end{cases}
$$

where $Q$ is any of the advected quantities, and $((\nabla Q))$ is a cell-centered numerical estimate of the gradient in $Q$. Notice that $A$ operates on the time-advanced volume fraction in Eq. (5.71), with the time-advanced velocity (that is, time-advanced in the predictor sense). In Eqs. (5.73)-(5.75) $A$ operates on $n$-time quantities with the $n$-time fluxing rate.

The operator $B_{p}$ is defined 


$$
B_{p}\left(Q^{n}\right)=\sum_{\alpha}\left(\nu_{t}^{(k)}\right)_{\alpha, f}^{n} \llbracket \nabla Q^{n} \rrbracket_{\alpha} \cdot \mathbf{S}_{\alpha}
$$

where $\llbracket \nabla Q^{n} \rrbracket_{\alpha}$ is a cell-side numerical estimate of $\nabla Q$ at cell face $\alpha$, and time level $n$. Calculation of both $((\nabla Q))$ and $\llbracket \nabla Q \rrbracket$ is described in Appendix A. Notice that the predictor diffusion operator involves only $n$-time quantities.

In the corrector step, for a typical cell, the mass equations are

$$
\left\langle\theta^{(k)}\right\rangle^{n+1}=\frac{1}{2}\left\{\left\langle\theta^{(k)}\right\rangle^{n}+\left\langle\theta^{(k)}\right\rangle^{\overline{n+1}}-\frac{\Delta t}{V} A\left(\left\langle\theta^{(k)}\right\rangle^{n+1}\right)\right\}
$$

and

$$
\left\langle\theta^{(k)}\right\rangle^{n+1}+\left\langle\theta^{(l)}\right\rangle^{n+1}=1,
$$

the momentum equation is

$$
\begin{aligned}
\left\langle\theta^{(k)} \mathbf{u}\right\rangle^{n+1}=\frac{1}{2}\left\{\left\langle\theta^{(k)} \mathbf{u}\right\rangle^{n}\right. & -\frac{\Delta t}{V} A\left(\left\langle\theta^{(k)} \mathbf{u}\right\rangle^{\overline{n+1}}\right) \\
& -\frac{\left.\Delta t\left\langle\theta^{(k)}\right)\right\rangle^{n+1}}{V} \sum_{\alpha}\left(\frac{1}{\rho^{(k)}}\langle p\rangle_{\alpha, b}^{n+1}-\phi_{\alpha, b}\right) \mathbf{S}_{\alpha} \\
& +\frac{\Delta t \nu^{(k)}}{V} \sum_{\alpha}\left\langle\theta^{(k)}\right\rangle_{\alpha, b}^{\overline{n+1}} \vec{D}_{\alpha}^{(k) \overline{n+1}} \cdot \mathbf{S}_{\alpha} \\
& \left.+\frac{\Delta t}{V} \sum_{\alpha} \vec{R}_{\alpha, b}^{(k) \overline{n+1}} \cdot \mathbf{S}_{\alpha}+\frac{1}{\rho^{(k)}} \mathbf{F}^{(k) n+1}\right\}
\end{aligned}
$$

the mean turbulence kinetic energy equation is

$$
\begin{aligned}
\left\langle k^{(k)}\right\rangle^{n+1} & =\frac{1}{2}\left\{\left\langle k^{(k)}\right\rangle^{n}+\left\langle k^{(k)}\right\rangle^{\overline{n+1}}-\frac{\Delta t}{V} A\left(\left\langle k^{(k)}\right\rangle^{\overline{n+1}}\right)\right. \\
& \left.+\frac{\Delta t}{V \sigma_{k}} B_{c}\left(\left\langle k^{(k)}\right\rangle^{\overline{n+1}}\right)+\Delta t\left[\left[P^{(k)}-\left\langle\epsilon^{(k)}\right\rangle+\frac{a^{(k)}}{\rho^{(k)}} F_{i}^{(k)}\left\langle u_{i}^{(l k)}\right\rangle\right]\right]^{\overline{n+1}}\right\}
\end{aligned}
$$

and the equation for the mean dissipation rate is

$$
\left\langle\epsilon^{(k)}\right\rangle^{n+1}=\frac{1}{2}\left\{\left\langle\epsilon^{(k)}\right\rangle^{n}+\left\langle\epsilon^{(k)}\right\rangle^{\overline{n+1}}-\frac{\Delta t}{V} A\left(\left\langle\epsilon^{(k)}\right\rangle^{\overline{n+1}}\right)\right.
$$




$$
\begin{aligned}
& +\frac{\Delta t}{V \sigma_{\epsilon}} B_{c}\left(\left\langle\epsilon^{(k)}\right\rangle^{\overline{n+1}}\right) \\
& +\Delta t\left[\frac{\left\langle\epsilon^{(k)}\right\rangle}{\left\langle k^{(k)}\right\rangle}\left[C_{1} P^{(k)}-C_{2}\left\langle\epsilon^{(k)}\right\rangle\right]+C_{3} C_{\mu} \frac{a^{(k) 3 / 2}}{\rho^{(k)}} \frac{\left|\left\langle\mathbf{u}^{(21)}\right\rangle\right|}{s} F_{i}^{(k)}\left\langle u_{i}^{(l k)}\right\rangle\right]^{\overline{n+1}} \\
& +\Delta t\left[2\left\langle\theta^{(k)}\right\rangle\left[\nu \mid \nu^{(k)}\left(\frac{1}{V} \sum_{\alpha} \llbracket \nabla\left\langle\mathbf{u}^{(k)}\right\rangle \rrbracket \cdot \mathbf{S}_{\alpha}\right)^{2}\right]^{\frac{n+1}{n}}\right\}
\end{aligned}
$$

where the corrector diffusion operator $B_{c}$ is

$$
B_{c}\left(Q^{\overline{n+1}}\right)=\sum_{\alpha}\left(\nu_{t}^{(k)}\right)_{\alpha, b}^{\overline{n+1}} \llbracket \nabla Q^{\overline{n+1}} \mathbb{Z}_{\alpha} \cdot \mathbf{S}_{\alpha}
$$

and $\llbracket \rrbracket^{\overline{n+1}}$ means evaluation at the predictor time level.

Equations (5.71) -(5.83) constitute the discretized form of (5.54)-(5.70). Supplemented with initial conditions and boundary conditions on the independent variables $\left\langle\theta^{(k)}\right\rangle,\left\langle\theta^{(k)} \mathbf{u}\right\rangle,\langle p\rangle,\left\langle k^{(k)}\right\rangle$, and $\left\langle\epsilon^{(k)}\right\rangle,(5.71)-(5.83)$ provide a practical means for the study of complicated mulimaterial flows. The specification of boundary and initial values on $\left\langle u^{(k)}\right\rangle$ and $\langle p\rangle$ is done following the rules on pressure and velocity given in the previous section. Since $\left\langle\theta^{(k)} \mathbf{u}\right\rangle=\left\langle\theta^{(k)}\right\rangle\left\langle\mathbf{u}^{(k)}\right\rangle$, the only additional rules needed are for $\left\langle\theta^{(k)}\right\rangle,\left\langle k^{(k}\right\rangle$, and $\left\langle\epsilon^{(k)}\right\rangle$ in order to complete the specification of boundary conditions and initialization.

The initial values of $\left\langle k^{(k)}\right\rangle$ and $\left\langle\epsilon^{(k)}\right\rangle$ throughout the computational domain are arbitrary, for problems in which a steady flow solution is required. In this case the final distribution is determined by the boundary values and by the physical competition between the processes of turbulence energy production, decay and diffusion. For time-unsteady problems the initial values of $\left\langle k^{(k)}\right\rangle$ and $\left\langle\epsilon^{(k)}\right\rangle$ are not necessarily arbitrary and must be established from prior knowledge of the initial flow conditions. Time-unsteady flows can be initialized from a steady-flow configuration, in a manner designed to simulate an actual laboratory experiment. For example, if the unsteady flow past an obstacle in a pipe is of interest, a sufficient length of pipe, upstream of the obstacle, should be provided to enable the development of a fixed distribution in velocity across the pipe before the flow encounters the obstacle.

The initialization of $\left\langle\theta^{(k)}\right\rangle$ is restricted only by the conditions*

* An exception to (5.84) occurs when a close-packing limit on one of the materials is enforced. In this case, the upper limit on $\langle\theta\rangle$ for that material is dictated to be a number less than unity, which corresponds to the volume fraction of closepacked solids. In this case a second pressure must be introduced into the equations, which is beyond the scope of this study. 


$$
0 \leq \theta^{(k)} \leq 1
$$

and

$$
\left\langle\theta^{(1)}\right\rangle+\left\langle\theta^{(2)}\right\rangle=1 .
$$

Boundary conditions of $\left\langle\theta^{(k)}\right\rangle$ can be expressed by the following simple rule. $A t$ an inflow boundary, $\left\langle\theta^{(k)}\right\rangle$ may have any specified value subject to conditions (5.84) and (5.85). At any othe boundary $\left\langle\theta^{(k)}\right\rangle$ satisfies the zero-gradient condition.

The zero-gradient condition means that $\left\langle\theta^{(k)}\right\rangle$ at the boundary is equal to $\left\langle\theta^{(k)}\right\rangle$ at the adjacent cell-center. The boundary condition rule for $\left\langle k^{(k)}\right\rangle$ is

At an inflow boundary $\left\langle k^{(k)}\right\rangle$ is specified. At a free-slip wall, $\left\langle k^{(k)}\right\rangle$ satisifes the zero gradient condition. At a no-slip wall $\left\langle k^{(\hat{\kappa})}\right\rangle=0$.

The rule for $\left\langle\epsilon^{(k)}\right\rangle$ is the same as for $\left\langle k^{(k)}\right\rangle$. Note that by definition $\nu_{t}^{(k)}=0$ at a no-slip wall.

This completes the detailed description of the numerical method used to solve the mean flow equations. The next section contains a description of the computer code used to carry out the solution for a variety of appl: itions that are presented in Chapter VI.

\section{FORTRAN COMPUTER CODE}

A FORTRAN computer program for conducting the numerical solution to the mean flow multimaterial equations, in two space dimensions, was prepared during this study. The main purpose of this section is to provide a brief description of the general properties of the code. Computer programs for multidimensional hydrodynamic studies are necessarily rather complex. In the present case, the complexity is compounded by both the multimaterial and the turbulence effects. Therefore before proceeding to utilize the computer program in circumstances for which the physical result may be unknown, it is important to gain some confidence that the code produces accurate results in problems for which solutions are known. The second purpose of this section is to demonstrate that the code carries out the numerical solution faithfully. This is done by reporting results in circumstances for which a solution is known, either experimentally or analytically.

A complete listing of the FORTRAN code developed for this study is provided in Appendix 4. The code is intended for use with the Cray FORTRAN compiler (Cray Research 1976), and as such makes use of a variety of techniques for optimized vector processing. The program is designed to be compact, readable and easily extended to handle more general sets of discrete multimaterial equation systems. The compactness is obtained mainly by the widespread use of multiplydimensioned arrays so that, for example, separate variables do not have to be 
defined for horizontal and vertical components of velocity. For tensor quantities, this produces a significant savings in the number of FORTRAN variables in the code. Readability is achieved in two ways. First, variable names are selected that emulate the meaning of each of the variables, and second a "modular" construction is used. "Modular" means that a separate subroutine is provided for calculation of each term in the equations representing a separate physical effect. For example, subroutine PRESAC uses the pressure $P R$ to perform the pressure acceleration in the momentum equation. The modularity also enhances the ability to extend the code beyond its present capabilities.

The data storage scheme used in the code is the multiregion data structure introduced by Baumgardner.* In two-dimensions, this scheme represents the logical domain as a collection of rectangular "regions" that are connected together through boundary conditions. (In three dimensions each region is a regular hexahedron.) During the computation, the regions are processed in succession, each computational time increment. The main advantage of this multiregion data structure is that of optimizing vectorization of the calculations performed in each cell of the mesh. Because the boundaries of each side of any given region are of one type, the multiregion data structure also expedites the setting of boundary conditions, in addition to improving the compactness of the code. (At present the implementation of the multiregion feature of the code listed in Appendix $\mathrm{C}$ is such that computations are limited to a single region.)

The two-dimensional code is configured to allow use of either a plane coordinate system or axisymmetric coordinates. In the latter case, the left side of the computational domain is considered to be an axis of rotation. One-dimensional problems are computed by simply specifying a single row or a single column of computational cells.

The multimaterial aspect of the code is generalized to enable an arbitrary number of materials. (A practical limitation exists, of course, that depends on the amount of memory space available.) For the problems considered in this study, the maximum number of materials used is two. Extension to larger numbers of materials is a straightforward task within the current construction of the code.

The composition of the code can be understood by examination of the first three subroutines that appear in the listing in Appendix C. The first routine is a brief glossary that defines the essential FORTRAN variables used in the program. The next routine is the main program whose function is simply to first call the input and initialization routines, then transfer control step-by-step to the third routine and finally to call the output routine. The third subroutine is where the real business of carrying out the computation takes place. This subroutine consists mainly of a two-pass $D O$ loop in which calls are made to a variety of more generic routines that perform various parts of the computation. For example subroutine

* Dr. John Baumgardner, Mail Stop B-216, Los Alamos National Laboraiory, Los Alamos, NM 87545. Unpublished work. 1986. 
$A D V E C T$ applies the advection operator to the first variable in its argument list. Each of the remaining subroutines are listed in alphabetical order, and have a short description of their function in a comment at the beginning of the routine.

The performance of this computer program, in terms of its ability to carry out the predictor-corrector solution scheme, will now be demonstrated by displaying the results from four calculations. These are time-unsteady Couette flow, steady laminar two-dimensional channel flow, turbulent channel flow and development of a two-dimensional Rayleigh-Taylor instability. The first three problems involve single-fluid flows and are selected as being typical test problems for which the solutions are well known. The Rayleigh-Taylor problem is a two-fluid flow with initially separated materials. By specifying a vanishingly small entity scale $s$, one can simulate the case of infinite $K$ so that interpenetration is prevented. Thus if the two fluids are initially separated, they will remain separated forever. This set of tesi problems is selected in order to test each of the various facets of the code, with the exception of its ability to compute interpenetrating flow problems. Such problems are the real target of the entire study and are the subject of Chapter VI.

The first test problem is chosen in order to demonstrate the ability of the solution scheme to accurately calculate time-unsteady flow problems. This unsteady flow problem involves the development of a velocity profile between parallel plates, one of which is impulsively set into motion in its own plane. This is called flow formation in Couette motion, for which the single-fluid conservation laws reduce to a diffusion equation, whose solution by series expansion is well known (Schlichting 1955,65 ). The numerical result for $R e=1$, based on the velocity of the wall and the wall spacing, is given by the solid lines in Fig. 8, where the series solution is shown by the symbols.

In this one-dimensional calculation, 10 cells are uniformly spaced across the channel. The hydrodynamic part of the computation required 0.975 central processing unit (CPU) seconds, on a Cray X-MP-4 computer, running in an interactive environment.

The second test calculation is the so-called hydrodynamic entry length problem, for flow in a circular tube. In this steady, two-dimensional, laminar flow problem, fluid enters a pipe with a uniform (slug) flow profile and develops toward the steady parabolic profile as the distance away from the entrance increases. The length required to reach $98 \%$ of the final centerline velocity is called the hydrodynamic entry length (Kays 1966, 60). Figure 9 is a plot of typical velocity vectors for the entry length problem at $R e=20$. Figure 10 is a log-log plot of the error versus the mesh spacing showing that the solution scheme behaves in a spatially secondorder accurate fashion. The computer time required for the case having 10 cells across the radius of the pipe and 40 cells along its length was 5.602 interactive CPU seconds.

The third test problem is the case of one-dimensional, steady turbulent flow in a channel at $R e=12,300$. This single-fluid circumstance is selected for comparison 


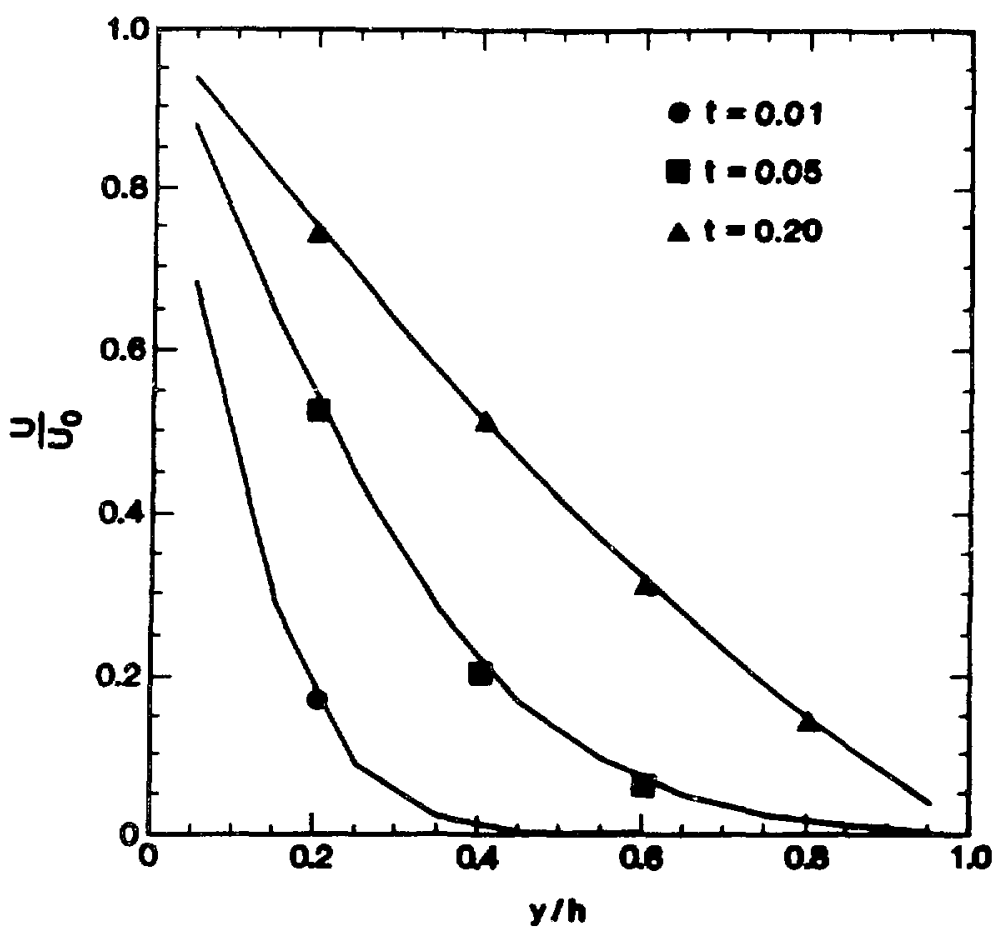

Figure 8. Flow formation in Couette motion. Solid line is the computed result. The symbols indicate the series solution.

to the experimental data of Laufer (1951). The computation is performed with a single column of 25 cells across the channel half-width, and specified-pressure boundary conditions on the channel ends, in order to simulate an infinitely long channel. The bottom wall is no-slip, and the top wall is free-slip, simulating a plane of s smmetry at the center of the channel. In this computation, a uniform initial flow is allowed to develop in time to a steady configuration. Figure 11 is a plot of the Reynolds stress $R_{12}$ and the mean flow velocity versus channel height.

The agreement shown in Fig. 11 demonstrates two things. The main feature of interest is that the $\left\langle k^{(k)}\right\rangle$ and $\left\langle\epsilon^{(k)}\right\rangle$ equations seem to be integrated properly by the computer code. The other feature is that the $k-\epsilon$ model for a single turbulent shear flow appears to be quite accurate. (This is of no particularly great consequence because the coefficients in the $k-\epsilon$ model for a singie-fluid are "tuned" for this type of flow.) The foregoing turbulent flow reached a steady flow velocity profile in about 600 seconds of problem (simulated) time. The calculation required 2500 time-steps that took 25.463 interactive CPU seconds to complete.

The final test problem is designed to test at least some facets of the multimaterial part of the computation. This is the so-called Rayleigh-Taylor instability problem, experimental data for which have been produced by Ratafia (1973). The initial conditions for Ratafia's experiment are shown in Fig. 12.

At time zero a uniform acceleration is applied causing the heavier fluid (water) 


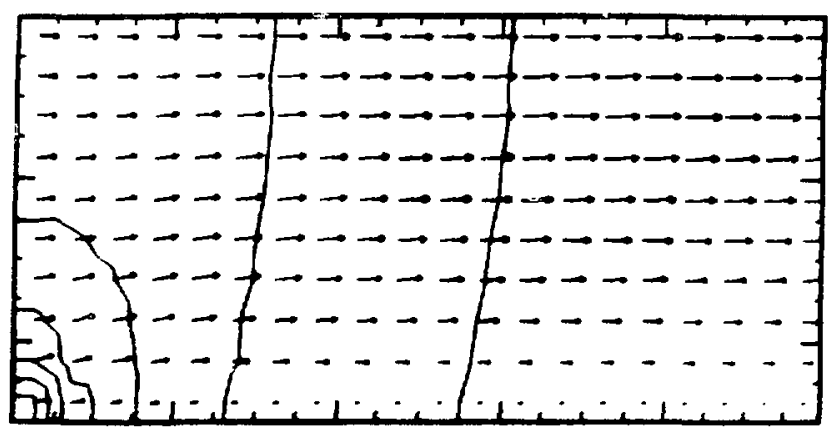

Figure 9. Hydrodynamic entry length problem. Typical velocity vectors, and contours of constant pressure.

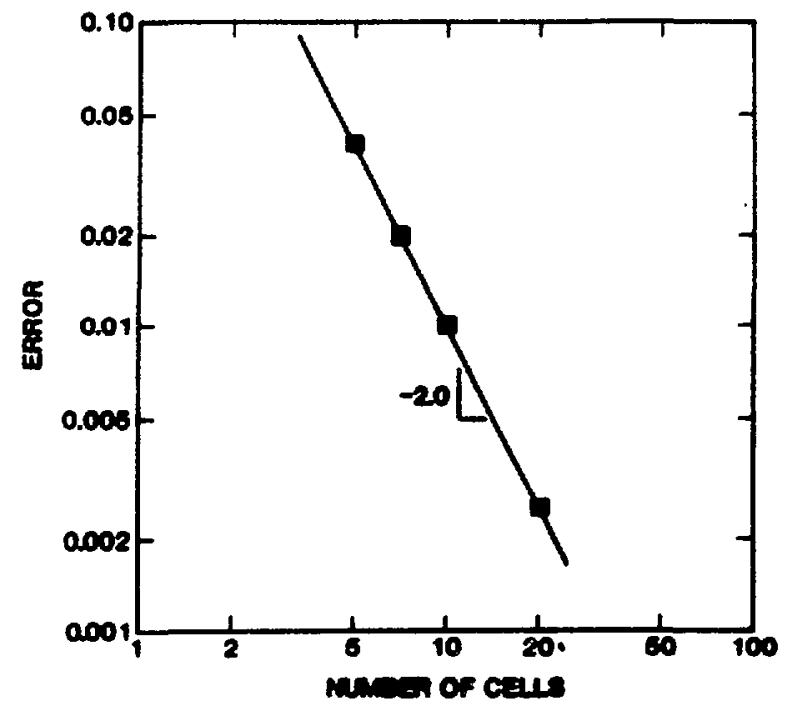

Figure 10. Hydrodynamic entry length problem. Error versus mesh spacing.

to begin displacing the lighter fluid (alcohol). Figure 13 is a time-sequence of volume fraction contours for the water, superimposed with velocity vectors of $\langle\theta u\rangle$ for the water.

The size and shape of the spike (downward-pointing water feature) in Fig. 13 at $t=0.65$, appear identical to the photographic data of Ratafia. This problem required 147 time-steps and 63.407 interactive CPU seconds to complete., The foregoing agreement for a two-dimensional, time unsteady (essentially nonviscous) flow problem, together with the results for the other three test problems, provide the confidence in the fidelity of the computer program needed to proceed onward to the applications discussed next. 

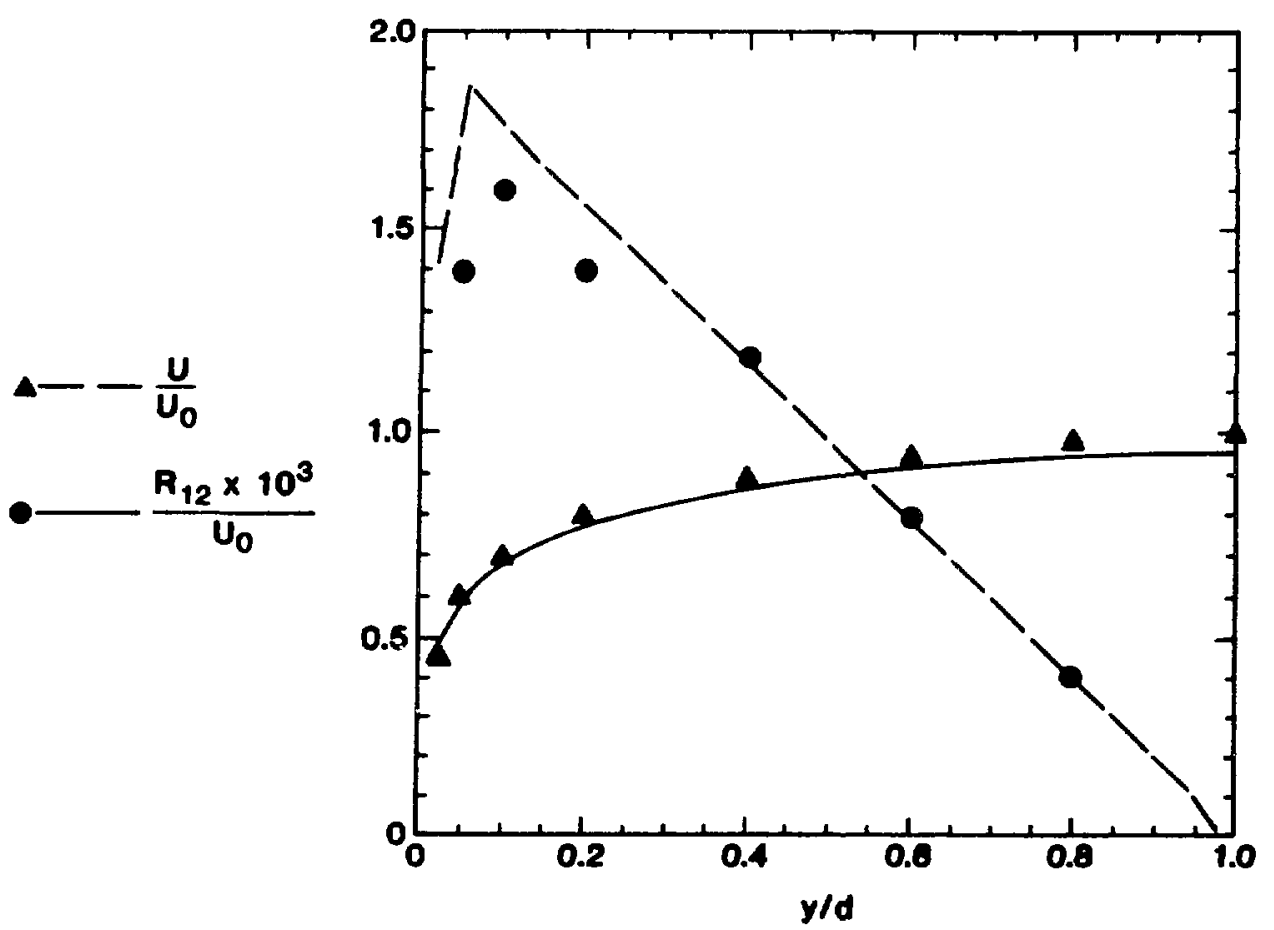

Figure 11. Flow in a channel at $R e=12,300$. Solid line is $R_{12} \cdot 10^{4}$ versus channel height, symbols are data due to Laufer (1951).

WATER, $P=1.00$

$0=-800 \mathrm{~cm} / \mathrm{e}$

ALCOHOL, $p=0.82$

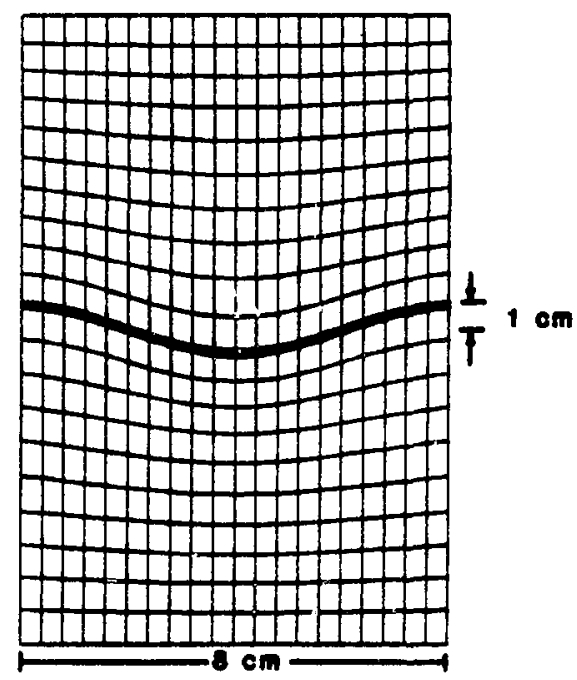

Figure 12. Initial conditions for the Rayleigh-Taylor irstability calculation. 


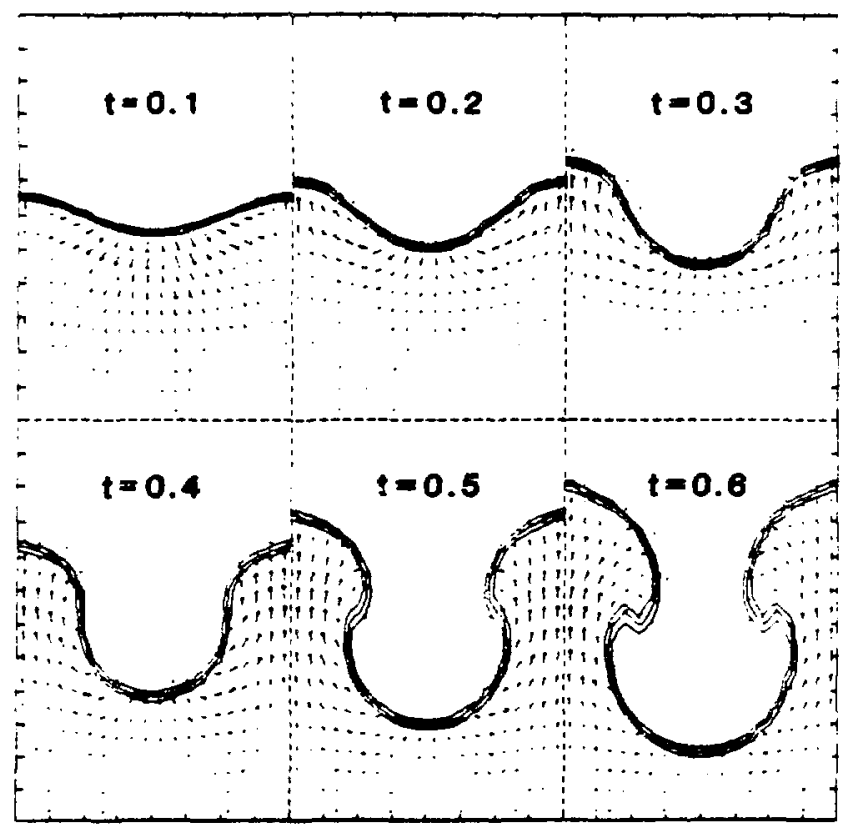

Figure 13. Rayleigh-Taylor instability ca'culation. Contours of water volume fraction $(0.4,0.5$, and 0.6$)$ superimposed over vectors of $\langle\theta u\rangle$ for the water.

\section{References Cited}

Amsden, A. A. and F. H. Harlow. 1978. K-TIF; A two-fluid computer code for downcomer flow dynamics. Los Alamos National Laboratory Report LA-6994.

Cray Research, Inc. 1976. Cray-1 and Cray X-MP computer systems. Mendota Heights: Cray Research.

Harlow, F. H. and J. E. Welch. 1965. Numerical Calculation of time-dependent viscous incompressible flow with free surface. Phys. Fluids. 8:2182-2189.

Hirt, C. W. 1968. Heuristic stability theory for finite-difference equations. $J$. Comput. Phys. 2:339-355.

Hirt, C. W., A. A. Amsden and J. L. Cook. 1974. An arbitrary LagrangianEulerian viuputing method for all flow speeds. J. Comput. Phys. 14:227253.

Hirt, C. W., B. D. Nichols and N. C. Romero. 1975. Sola-A numerical solution alogrithm for transient fluid flows. Los Alamos National Laboratory Report LA-5852.

Kashiwa, B. A. 1986a. A generalized MAC method for incompressible fluid flow. Los Alamos National Laboratory Report LA-10853-MS. 
1986b. A predictor-corrector method for calculation of incompressible fluid flow dynamics. Los Alamos National Laboratory report LA-10854-MS.

Kays, W. M. 1966. Convective heat and mass transfer. New York: McGraw-Hill.

Laufer, J. 1951. Investigation of turbulent flow in a two-dimensional channel. National Advisory Committee for Aeronautics report 1053.

Lax, P. D. and B. Wendroff. 1964. Difference schemes for hyperbolic equations with high order of accuracy. Comm. on Pure and Appl. Math. 17:381-398.

MacCormack, R. W. 1969. The effect of viscosity in hypervelocity impact cratering. AIAA Paper 69-354.

Ratafia, M. 1973. Experimental investigation of Rayleigh-Taylor instability. Phys. Fluids. 16:1207-1210.

Roache, P. J. 1972. Computational fluid dynamics. Albuquerque: Hermosa.

Schlichting, H. 1955. Boundary layer theory. J. Kestin translator. New York: McGraw-Hill.

van Leer, B. 1977. Towards the ultimate conservative difference scheme. IV. A new approach to numerical convection. J. Comput. Phys. 23:276-299.

Von Neumann, J. and R. D. Richtmyer. 1950. A method for the numerical calculation of hydrodynamic shocks. J. Applied Phys. 21:232-237.

Woodward, P. and P. Collela. 1984. The numerical simulation of tw -dimensional fluid flow with strong shocks. J. Comput. Phys. 54:115-173. 


\section{Chapter VI}

\section{Applications}

Here the multimaterial $k-\epsilon$ theory introduced in Chapters II-IV is applied to a set of problems in engineering for which experimental data exist. The purpose of this is to demonstrate the strengths and weaknesses of the theory in its present state of development. To accomplish this, three problems are considered. These are:

1. Calculation of the drag force on a single sphere in a uniform flow.

2. Calculation of the settling rate in a uniform suspension.

3. Calculation of the velocity profiles in the pneumatic transport of solid particles in a pipe.

The first problem is considered in Sec. 1 and reveals one of the powerful attributes of the new theory. By examining the special case of a single sphere in a uniform flow, the equations of motion can be reduced to an expression for the drag coefficient as a function of relative Reynolds number. This analytic result compares very well with experimental data over a wide range of relative Reynolds numbers. Thus the fundamental theoretical considerations embodied in the theory provide a way of obtaining what has been previously only available from careful experimentation or detailed multidimensional numerical simulations.

The second problem goes one step forward and shows that not only is the correct single-body drag force contained in the theory, but so is the correct manybody drag force. In Sec. 2, the settling speed in a uniform suspension of solid spheres is computed over a very wide range in values of solids volume fraction. The good comparison to experimental data for sedimentation speed at various relative Reynolds numbers provides a strong indication that the elements composing the new theory are correct.

In Sec. 3, a pneumatic transport problem is considered. In this problem, the effect of dispersed solid particles on the velocity profile of a turbulent flow of air in a vertical pipe is simulated. Comparison of computed results to experimental data indicate that many essential features of the interaction between the air and spherical particles are well represented. Some features are less well represented, indicating that the theory could benefit from additional development.

\section{DRAG FORCE ON A SINGLE SPHERE}

Consider a single sphere rigidly fixed in a fluid of density $\rho$, flowing uniformly with speed $u$ relative to the sphere. The magnitude of the drag force on the sphere $F$, per unit volume of the sphere, can be written 


$$
F=\frac{3}{8} \frac{\rho u^{2}}{s} C_{d}
$$

which effectively defines the drag coefficient $C_{d}$, where $s$ is the radius of the sphere. Let $\nu$ be the kinematic viscosity of the fluid. Dimensional considerations dictate that $C_{d}$ must be a function of the relative Reynolds number, $R e=2 s u / \nu$, only. For $R e \ll 1$ Stokes' result

$$
C_{d}=\frac{24}{R e}
$$

is valid. For $R e>1$ one must resort to careful experimentation or numerical computation of the complete flow field in order to establish the force, and therefore $C_{d}$. The goal here is to demonstrate that $C_{d}$ is contained within the multimaterial $k-\epsilon$ theory.

In this problem the flow domain of interest is exterior to the sphere so that only the average equations for the fluid need to be considered. Because the flow is time steady and there are no gradients in the average quantities, the equations for turbulence energy and decay rate reduce to

$$
\epsilon=\frac{F u}{\rho}
$$

and

$$
C_{2} \frac{\epsilon^{2}}{k}=C_{3} C_{\mu} \frac{u}{s}\left(\frac{F u}{\rho}\right)
$$

where the expectation bars and material number have been dropped. By definition the root mean square velocity fluctuatior. $q$ is $\left(\frac{2}{3} k\right)^{1 / 2}$. Using (6.3) and (6.4) this is

$$
q=\left(\frac{2}{3} \frac{C_{2}}{C_{3}} \frac{F u}{C_{\mu} \rho}\right)
$$

Suppose the expression

$$
C_{d}=\frac{24}{R e}+\beta_{1} \frac{q}{u}
$$

is contained in the exchange force using Eq. (3.50), in some approximation. (This will be shown to be true later.) Inserting the foregoing expression for $q$ and using (6.1), yields

$$
C_{d}-\beta_{1} A C_{d}^{1 / 2}-\frac{24}{R e}=0
$$

where 


$$
A=\left(\frac{1}{4} \frac{C_{2}}{C_{3}} \frac{1}{C_{\mu}}\right)^{1 / 2}
$$

is a fixed constant. Notice that $\beta_{1}$ and $C_{3}$ are unknown constants. A unique relation between these constants can be established by referencing the experimental data. For this, consider

$$
\lim _{R \rightarrow \infty} C_{d}=\frac{1}{2}
$$

The basis for this choice will be discussed shortly. Using (6.6) the relationship

$$
\frac{\beta_{1}}{C_{3}^{1 / 2}}=0.306
$$

can be established, in which the values $C_{2}=1.920$ and $C_{\mu}=0.090$ (Launder and Spalding 1974) have been used. The coefficient $C_{3}$ is expected to be near unity. Using $C_{3}=1.000$, Eq. (6.5) can be solved for $C_{d}(R e)$. The result is displayed in Fig. 14, together with the so-called "standard" drag curve (Clift, Grace and Weber 1978, 111).

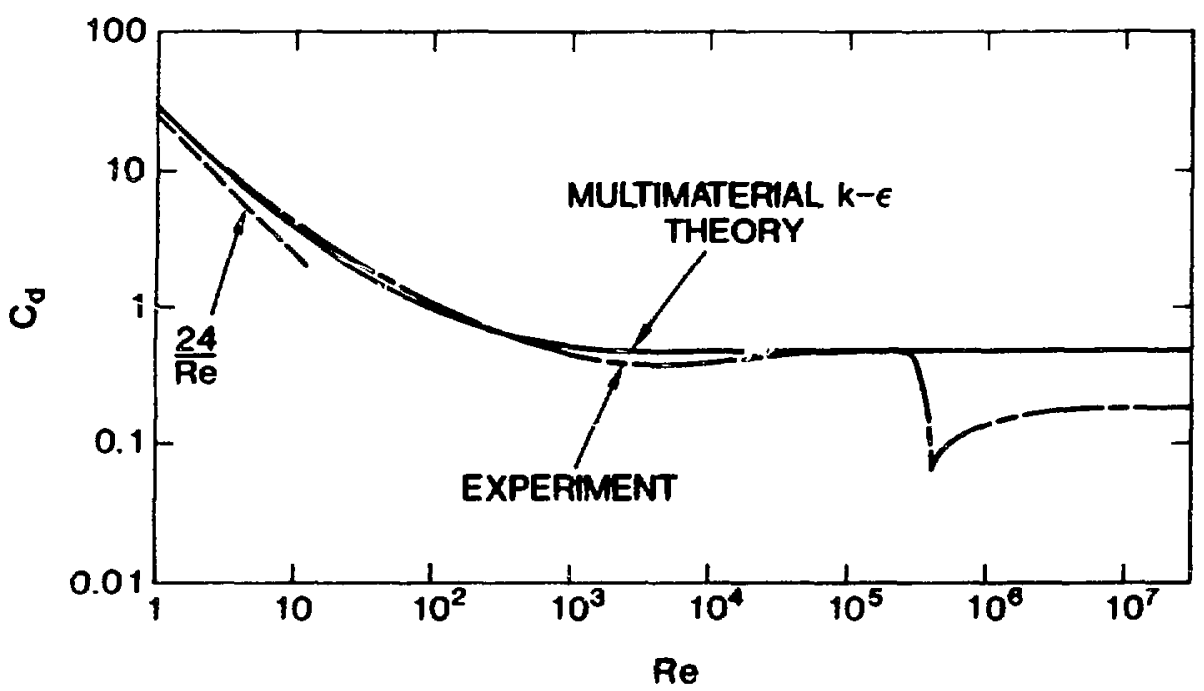

Figure 14. Drag coefficient for a single fixed sphere in a uniform flow. Comparison of the multimaterial theory with experimental data.

Notice that for $R e<3 \cdot 10^{5} \mathrm{Eq}$. (6.5a) produces $C_{d}$ quite accurately. At $R e \approx 3 \cdot 10^{5}$ (called the critical Reynolds number) the experimental drag coefficient undergoes a sudden decrease from 0.50 to about 0.20 and remains essentially constant with increasing $R e$. This sudden decrease in $C_{d}$ is associated with the transition from 
a laminar to a turbulent boundary layer on the sphere. The attendant change in the structure of the boundary layer causes the point of flow separation to move downstream on the sphere's surface reducing the cross-sectional area of the wake, and therefore the drag force.

In many multiphase flows, the apperarance of a critical Reynolds number effect is unlikely. There are at least two reasons for this. The first is that, in general, the dispersed entities are not perfect spheres, but rather some collection of irregular fragments (such as sand, pulverized coal or wheat grain). The second reason is that, for many-body circumstances, any given entity will encounter a nonuniform flow field that is set up by the flow past upstream entities. This gives the continuous fluid a pre-existing turbulence that tends to both raise the drag force on a single entity and diminish the critical Reynolds number effect (Clift, Grace and Weber 1978, 268). Hence the choice made in Eq. (6.6) is made on the assumption that a critical Reynolds number effect is absent.

The result in Fig. 14 can be understood on the following physical basis. The presence of an obstacle in a uniform flow causes a disturbance. This disturbance results in the transfer of kinetic energy from the ordered, uniform motion into disordered motion in the wake of the obstacle. The rate of this transfer is given by Eq. (6.3) which simply expresses the balance between production of fluctuational kinetic energy and its rate of destruction by viscous dissipation. The creation of fluctuational energy in the flow is accompanied by an enhanced rate of decay, because of the creation of small-scale fluid motion.

The balance between creation and destruction of dissipation rate is given by Eq. (6.4). The key facet of this model equation is the choice of the inverse time scales. For the rate of destruction, the inverse time scale is $C_{2} \epsilon / k$ as is usual in single-material $k-\epsilon$ theory. Based on the result in Fig. 14, the relevant inverse time scale for the creation of dissipation rate is $C_{3} C_{\mu} u / s$. (Note that $C_{3} q / s$ is almost as good for the single sphere problem, but the form given in Eq. (6.4) is a better choice, as demonstrated in the next section.)

The flow energy deposited in the turbulent fluctuations enters into the drag force on the body by introducing an asymmetry in the velocity statistics in the flow. This asymmetry is accounted for in the momentum exchange coefficient, a part of which is given by Eq. (6.5). Equation (6.5) expresses the magnitude of asymmetric effects as the sum of a viscous force and local (short-range) turbulence in the fluid. The third part, which is not relevant in this case and is therefore omitted, represents the effects of a pre-existing turbulence in the fluid (nonuniform flow).

Hence the drag force on a single sphere is contained within the multimaterial $k-\epsilon$ theory mainly as a consequence of fluctuational kinetic energy considerations. Deviations of $C_{d}$ from the experimental data exist because the detailed nature of the boundary layer on the sphere has been neglected in the developments. Nevertheless, the result of Fig. 14 is useful for a great many engineering purposes, 
and provides confidence in the potential for validation of the postulates contained in the theory. This is provided of course that Eq. (6.5) can be justified, which is addressed in the next section.

\section{SEDIMENTATION OF A SUSPENSION}

Consider now a vertical tube filled with a liquid initially having solid spherical particles randomly distributed throughout. The solid particles have a microscopic density $\rho^{(2)}$ larger than that of the liquid $\rho^{(1)}$, and a radius that is much smaller than that of the tube. The gravitational acceleration causes the dispersed particles to begin falling in the liquid, and the incompressibility of the system requires that the liquid begin to rise. Very quickly a time steady flow is established in which a layer of pure stationary liquid exists at the top of the tube, and a layer of close-packed spheres (filled with liquid) rests at the bottom of the tube.

This is the experimental configuration of Richardson and Zaki (1954), whose studies were motivated by the use of sedimentation processes in chemical engineering. They observed that the thickness of the top and bottom layers increased linearly in time, indicating the existence of a constant settling speed for the suspension. Richardson and Zaki referred to the division between the upper pure liquid layer and the uniform mixture as the "sludge line". They called the rate at which the sludge line descended the sedimentation velocity $V_{c}$. By changing the number of spheres in the tube, the variation in $V_{c}$ with the volume fraction of liquid $\left\langle\theta^{(1)}\right\rangle$ could be determined. Using liquids such as water mixed with glycerol and by varying the size of the dispersed spheres, Richardson and Zaki were able to measure the variation in $V_{c}$ with $\left\langle\theta^{(1)}\right\rangle$ for a variety of conditions characterized by the quantity $R e_{0}$ defined

$$
R e_{0}=\frac{2 s V_{0}}{\nu^{(1)}}
$$

This is a Reynolds number based on the free-fall velocity $V_{0}$ computed assuming Stokes law

$$
V_{0}=\frac{2}{9} \frac{g \Delta \rho s^{2}}{\rho^{(1)} \nu^{(1)}}
$$

where $g$ is the magnitude of the gravitational acceleration, $s$ is the radius of the sphere, $\nu^{(1)}$ is the liquid kinematic viscosity, and $\Delta \rho=\rho^{(2)}-\rho^{(1)}$. For $R e_{0}>0.2$, Richardson and Zaki found $V_{0}$ by observing the descent of a single sphere.

A significant finding of the Richardson and Zaki experiments is that the relationship of $\log V_{c}$ to $\log \left\langle\theta^{(1)}\right\rangle$ is approximately linear, with $V_{c}$ approaching $V_{0}$ as $\left\langle\theta^{(1)}\right\rangle$ approaches unity. Therefore these workers were able to report their data as the slope of the line in $\log V_{c}-\log \left\langle\theta^{(1)}\right\rangle$ coordinates, intercepting $\log \left\langle\theta^{(1)}\right\rangle=0$ at $\log V_{0}$. 
The goal here is to demonstrate that the multimaterial $k-\epsilon$ theory predicts the correct variation in $V_{c}$ with $\left\langle\theta^{(1)}\right\rangle$. To accomplish this, $V_{c}$ must first be related to the magnitude of the relative mean velocity $u$. Then the momentum equations and the equations for the turbulence energy and decay rate can be combined in a way that reveals a prediction for the variation in $V_{c}$ with $\left\langle\theta^{(1)}\right\rangle$.

Clearly the rate at which the sludge line descends is just the negative of the vertical component of the particle field average velocity. Thus $V_{c}=-\left\langle u^{(2)}\right\rangle$. This is because the average force per unit volume on material two increases with increasing $\left\langle\theta^{(2)}\right\rangle$. Hence any tendency for particles to "pile up" at the sludge line is accompanied by an increase in the force that retards their motion. Conversely any tendency for particles to lag behind the sludge line is accompanied by a lower $\left\langle\theta^{(2)}\right\rangle$ and therefore a decrease in the resistive force allowing the particles to speed up. The result is a stable configuration in the sludge line that resembles a discontinuity in the volume fraction, travelling at the average speed of the falling particles.

Thus the first problem is to relate the relative mean velocity to the mean particle velocity. This can be accomplished by using the multimaterial incompressibility condition, Eq. (2.19b). Considering a small control volume in the uniform area of the suspension, away from the wall of the tube (where presumably a boundary layer exists), Eq. (2.19b) implies that

$$
\left\langle u^{(1)}\right\rangle\left\langle\theta^{(1)}\right\rangle+\left\langle u^{(2)}\right\rangle\left\langle\theta^{(2)}\right\rangle=0
$$

where $\left\langle u^{(k)}\right\rangle$ is the average vertical component of velocity for material $k$. The horizontal component is, on the average, zero. Since $u=\left\langle u^{(2)}\right\rangle-\left\langle u^{(1)}\right\rangle$ and $\left\langle\theta^{(2)}\right\rangle=1-\left\langle\theta^{(1)}\right\rangle$, this can be rearranged to give

$$
-\left\langle u^{(2)}\right\rangle=\left\langle\theta^{(1)}\right\rangle u=V_{c}
$$

as needed.

Away from the tube wall, the gradients in the mean quantities are assumed to be small. Neglecting terms proportional to spaital gradients, the time steady momentum equation for material two (vertical component) is

$$
F-\left\langle\theta^{(2)}\right\rangle \Delta p+\rho^{(2)}\left\langle\theta^{(2)}\right\rangle g=0
$$

where $F$ is the vertical component of the exchange force per unit volume acting on material two, and $\Delta p$ is the vertical component of the pressure gradient. The vertical hydrostatic pressure gradient is just $\Delta p=\left(\rho^{(1)}\left\langle\theta^{(1)}\right\rangle+\rho^{(2)}\left\langle\theta^{(2)}\right\rangle\right) g$ so that this can be rearranged to give

$$
g \Delta \rho\left\langle\theta^{(1)}\right\rangle\left\langle\theta^{(2)}\right\rangle=F
$$

where again $\Delta \rho=\rho^{(2)}-\rho^{(1)}$.

The next step is to eliminate $F$ from (6.11) using the multimaterial equation for $\left\langle k^{(k)}\right\rangle$ and $\left\langle\epsilon^{(k)}\right\rangle$. Let us consider the force in the form 


$$
F=\frac{3}{8}\left\langle\theta^{(1)}\right\rangle\left\langle\theta^{(2)}\right\rangle\left[\rho^{(1)} \frac{24}{R e}+\beta_{2} C_{\mu} \frac{\left[\mu_{t}\right]}{u s}\right] \frac{u^{2}}{s}
$$

from which the term representing the short-range part of the exchange force is omitted and $R e=2 s u /[\nu]$. (The short-range part turns out be be negligible for the Richardson and Zaki experiments in which $R e_{0}<0.2$, as will be seen shortly.) To eliminate $\left[\mu_{t}\right]$ from (6.12) the time steady, zero gradient energy and decay rate equations are required. These are

$$
\left\langle\epsilon^{(k)}\right\rangle=a^{(k)} \frac{F u}{\rho^{(k)}}
$$

and

$$
C_{2} \frac{\left\langle\epsilon^{(k)}\right\rangle^{2}}{\left\langle k^{(k)}\right\rangle}=C_{3} C_{\mu} \sqrt{a^{(k)}} \frac{u}{s}\left(a^{(k)} \frac{F u}{\rho^{(k)}}\right)
$$

where as before

$$
a^{(1)}=\frac{\rho^{(2)}}{\gamma \rho^{(1)}+\rho^{(2)}}
$$

and

$$
a^{(2)}=\frac{\gamma \rho^{(1)}}{\gamma \rho^{(1)}+\rho^{(2)}}
$$

are the respective fraction of the total fluctuational energy produced (by interpenetration) that goes into field one and two, and

$$
\gamma=\frac{\rho^{(2)}}{\rho^{(1)}} \frac{\left\langle k^{(2)}\right\rangle}{\left\langle k^{(1)}\right\rangle}
$$

Equation (6.16) is motivated by the idea that materials one and two are in a state of "pressure" equilibrium in the suspension. The turbulence pressure is $\rho^{(k)} V^{(k)}\left\langle k^{(k)}\right\rangle$ so that

$$
\rho^{(2)} V^{(2)}\left\langle k^{(2)}\right\rangle=\rho^{(1)} V^{(1)}\left\langle k^{(1)}\right\rangle
$$

and Eq. (6.16) follows. Thus for the single sphere problem $\gamma=1$ and $a^{(1)}=1$. There is no experimental evidence for the validity of $\mathbb{E q}$. (6.16), so that the prescription must be regarded as another postulate. The results that follow suggest that (6.16) is at least quite feasible.

Equation (6.13) can be used in $\mathbb{E} q$. (6.14) to produce 


$$
\frac{\left\langle k^{(k)}\right\rangle}{\left\langle\epsilon^{(k)}\right\rangle}=\frac{C_{2}}{C_{3}} \frac{s}{C_{\mu} u} \frac{1}{\sqrt{a^{(k)}}}
$$

so that

$$
\nu_{t}^{(k)}=\left(\frac{C_{2}}{C_{3}}\right)^{2} \frac{s^{2}}{C_{\mu} u} \frac{F}{\left\langle\theta^{(k)}\right\rangle \rho^{(k)}}
$$

where (6.13) has been used again. Now the expected turbulent viscosity is

$$
\begin{aligned}
{\left[\mu_{t}\right] } & =\left\langle\theta^{(1)}\right\rangle \rho^{(1)} \nu_{t}^{(1)}+\left\langle\theta^{(2)}\right\rangle \rho^{(2)} \nu_{t}^{(2)} \\
& =2\left(\frac{C_{2}}{C_{3}}\right)^{2} \frac{s^{2}}{C_{\mu} u} F .
\end{aligned}
$$

Entering this into Eq. (6.12) and combining with (6.11) produces the result

$$
V_{c}=V_{0}\left[1-2\left\langle\theta^{(1)}\right\rangle\left\langle\theta^{(2)}\right\rangle \beta_{2}\left(\frac{C_{2}}{C_{3}}\right)^{2}\right]
$$

where Eq. (6.10) has been used, and where $V_{0}$ is the Stokes free-fall velocity given by Eq. (6.9).

Expression (6.17) exhibits the main essence of the long-range part of the exchange force. As $\left\langle\theta^{(1)}\right\rangle$ departs from unity, the product $\left\langle\theta^{(1)}\right\rangle\left\langle\theta^{(2)}\right\rangle$ increases causing $V_{c}$ to decrease. This can be thought of as resulting from an increase in the pre-existing level of turbulence in the fluid and in the particles upstream of any given isolated particle. The increase in fluid turbulence is caused by the tortuous path that a fluid element must take in moving through the particles, and increases with decreasing $\left\langle\theta^{(1)}\right\rangle$.

Note that the unknown coefficients in Eq. (6.17) form a group, so that only a relationship among them can be determined by comparison to experimental data. For $R e_{0} \ll 1$, where (6.17) can be expected to be valid, the combination

$$
\frac{\beta_{2}}{C_{3}^{2}}=1.333
$$

is found to give accurate results, by comparison to the Richardson and Zaki data (when again $C_{2}=1.920$ is assumed to be known from single-phase turbulence theory). If $R e>1$ then flow separation becomes significant and one must use the full drag force

$$
F=\frac{3}{8}\left\langle\theta^{(1)}\right\rangle\left\langle\theta^{(2)}\right\rangle\left[\rho^{(1)} \frac{24}{R e}+\frac{\beta_{1} \rho^{(1)} a^{(1)}}{\left(\left\langle\theta^{(1)}\right\rangle\left\langle\theta^{(2)}\right\rangle\right)^{1 / 2}} \frac{[q]}{u}+\beta_{2} C_{\mu} \frac{\left[\mu_{t}\right]}{u s}\right] \frac{u^{2}}{s}
$$


together with Eqs. (6.13)-(6.16) and (6.11). This forms a nonlinear system of equations that is easily solved precisely by Newton iteration. A short FORTRAN program for obtaining the solution is given in Appendix D.

To illustrate the performance of the foregoing equations, consider the six cases from the sedimentation experiments of Richardson and Zaki (1954) listed in Table 1. Notice that these cases contain free-fall relative Reynolds numbers that span five orders of magnitude. Comparison with the theory is given in Fig. 15. There it is clear that both the magnitude of $V_{c}$ and the linear character of the variation is contained accurately in the theory. This is true over a range of particle loadings from zero to nearly close-packed solids. (This impressive result suggests that the multimaterial $k-\epsilon$ theory may even be useful for prediction of permeabilities in porous media.)

Table 1. Conditions for six of the sedimentation experiments of Richardson and Zaki (1954).

\begin{tabular}{lrrllll}
\hline Run & $\rho^{(1)}$ & $\begin{array}{c}\rho^{(2)} \\
\left(\mathrm{g} / \mathrm{cm}^{3}\right)\end{array}$ & $\begin{array}{l}\nu^{(1)} \\
\left(\mathrm{cm}^{2} / \mathrm{s}\right)\end{array}$ & $\begin{array}{l}s \\
(\mathrm{~cm})\end{array}$ & $\begin{array}{l}V_{0} \\
(\mathrm{~cm} / \mathrm{s})\end{array}$ & $R e_{0}$ \\
\hline $1,3,4$ & 2.745 & 2.976 & $6.70 \cdot 10^{-3}$ & $5.15 \cdot 10^{-2}$ & 2.48 & $3.83 \cdot 10^{+1}$ \\
$10,11,13$ & 2.170 & 2.623 & $7.43 \cdot 10^{-3}$ & $1.15 \cdot 10^{-2}$ & $6.53 \cdot 10^{-1}$ & 2.02 \\
$\mathrm{~N}, \mathrm{O}$ & 2.170 & 2.923 & $7.43 \cdot 10^{-3}$ & $4.80 \cdot 10^{-3}$ & $2.34 \cdot 10^{-1}$ & $3.03 \cdot 10^{-1}$ \\
$\mathrm{~A}, \mathrm{~B}, \mathrm{C}$ & 1.001 & 1.058 & $1.53 \cdot 10^{-2}$ & $9.05 \cdot 10^{-3}$ & $6.64 \cdot 10^{-2}$ & $7.89 \cdot 10^{-2}$ \\
$\mathrm{~K}, \mathrm{~L}$ & 1.208 & 2.923 & $5.13 \cdot 10^{-1}$ & $4.80 \cdot 10^{-3}$ & $1.39 \cdot 10^{-2}$ & $2.61 \cdot 10^{-4}$ \\
$\mathrm{P}, \mathrm{Q}$ & 1.034 & 1.058 & $2.01 \cdot 10^{-1}$ & $9.05 \cdot 10^{-3}$ & $2.06 \cdot 10^{-3}$ & $1.85 \cdot 10^{-4}$ \\
\hline \multicolumn{7}{l}{ ("Run" is the experiment identification used by Richardson and Zaki.) } \\
\hline
\end{tabular}

Now observe that the force in Eq. (6.19) is exactly the force given by the momentum exchange function (3.50), with two morifications. The first is the factor $\left(\left\langle\theta^{(1)}\right\rangle\left\langle\theta^{(2)}\right\rangle\right)^{1 / 2}$ in the denominator of the separation term. This is necessary in order for the expression to limit to Eq. (6.5) in the single sphere case. The other modification is the factor of $C_{\mu}$ in the small-scale rotation ter n. This appears in order to cancel with a similar factor in the $\left\langle\epsilon^{(k)}\right\rangle$ equation in arriving at (6.17). Hence it is clear that the momentum exchange function has the final form (for the scalar part)

$$
\begin{aligned}
K= & \rho^{(1)}\left\langle\theta^{(1)}\right\rangle\left\langle\theta^{(2)}\right\rangle \\
& \left(\frac{\beta_{1}^{\prime} a^{(1)}}{\left(\left\langle\theta^{(1)}\right\rangle\left\langle\theta^{(2)}\right\rangle\right)^{1 / 2}} \frac{[q]}{\left\langle\mathbf{u}^{(21)}\right\rangle \mid}+\beta_{2}^{\prime} C_{\mu} R e_{t}^{-1}+\frac{9}{2} R e_{v}^{-1}\right) \frac{\left|\left\langle\mathbf{u}^{(l k)}\right\rangle\right|}{s},
\end{aligned}
$$

where $\beta_{1}^{\prime}=\frac{3}{8} \beta_{1}$ and $\beta_{2}^{\prime}=\frac{3}{8} \beta_{2}$.

Thus another significant piece of evidence for the validity of the multimaterial $k-\epsilon$ theory exists in the sedimentation results. The next section contains one more 


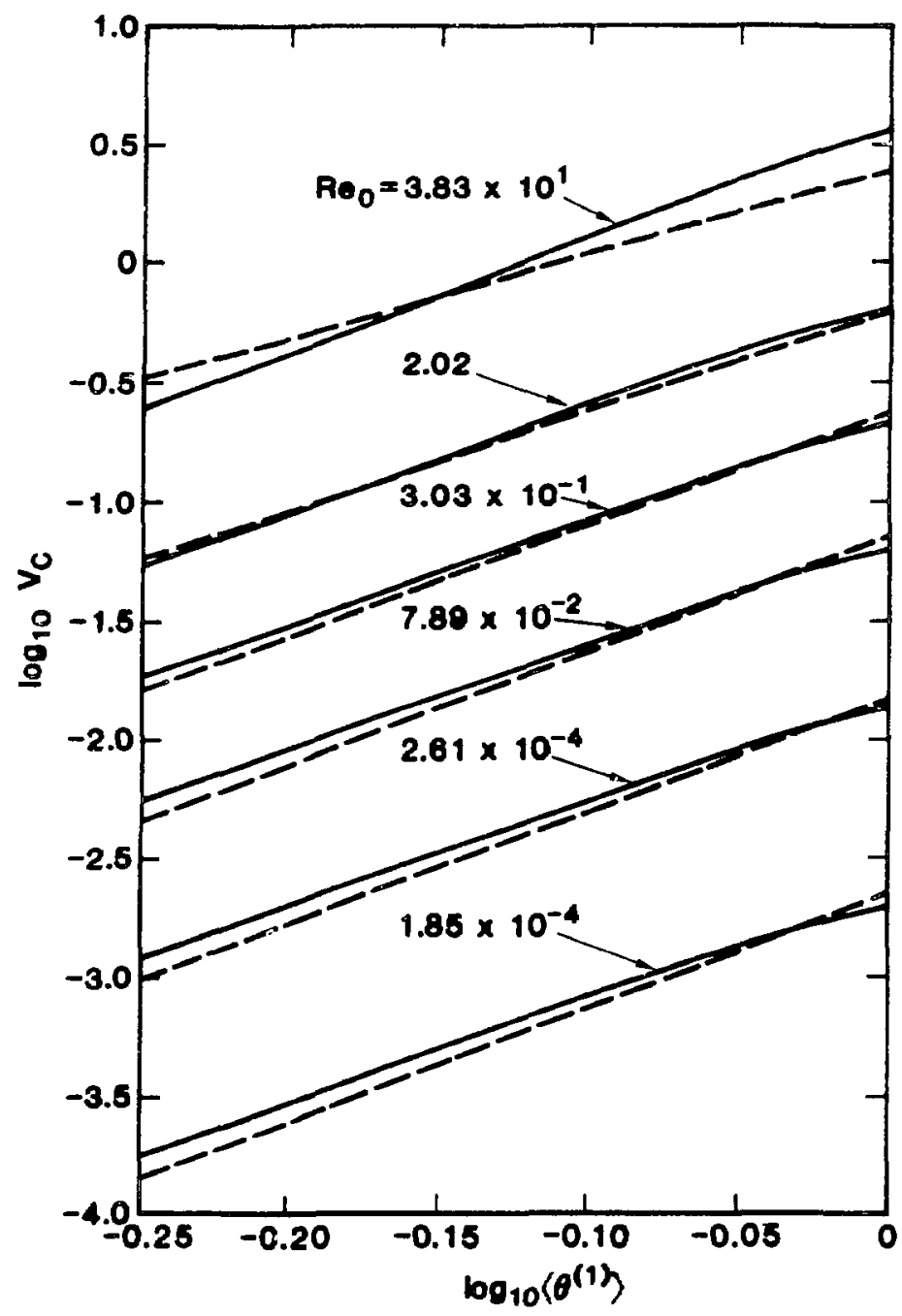

Figure 15. Sedimentation speed versus liquid volume fraction. Comparison of the theory with the experimental data of Richardson and Zaki (1954).

important circumstance by which to gauge the utility of the ideas contained in this thesis.

\section{PNEUMATIC TRANSPORT IN A PIPE}

Here attention is directed to the fully developed flow of air, loaded with spherical particles, travelling upward in a vertical pipe. The experimental data of Lee and Durst (1982) are typical of this pnuematic transport situation. These workers measured the mean velocity of both the carrier air and the transported particles at various radial locations in a pipe, using a laser-Doppler-anemometer. Figure 16 contains the main velocity profile data, and a brief summary of the 
test conditions from the Lee and Durst experiments. The pipe Reynolds number based on the pipe diameter of $4.18 \mathrm{~cm}$ and a nominal centerline velocity of 600 $\mathrm{cm} / \mathrm{s}$ is 17,300 . Notice that the velocity profiles are quite flat over a portion of the pipe nearest the center. This indicates that the flow near the pipe center is approximately in a state of "uniform fluidization", which means that the volume fraction of particles is nearly constant. Close to the wall however, Lee and Durst observed a thin region that was essentially free of particles. This region is presumed to be maintained as a result of the very steep gradient in the air velocity at the wall, which is known to cause a force perpendicular to the average relative motion (the Saffman force).
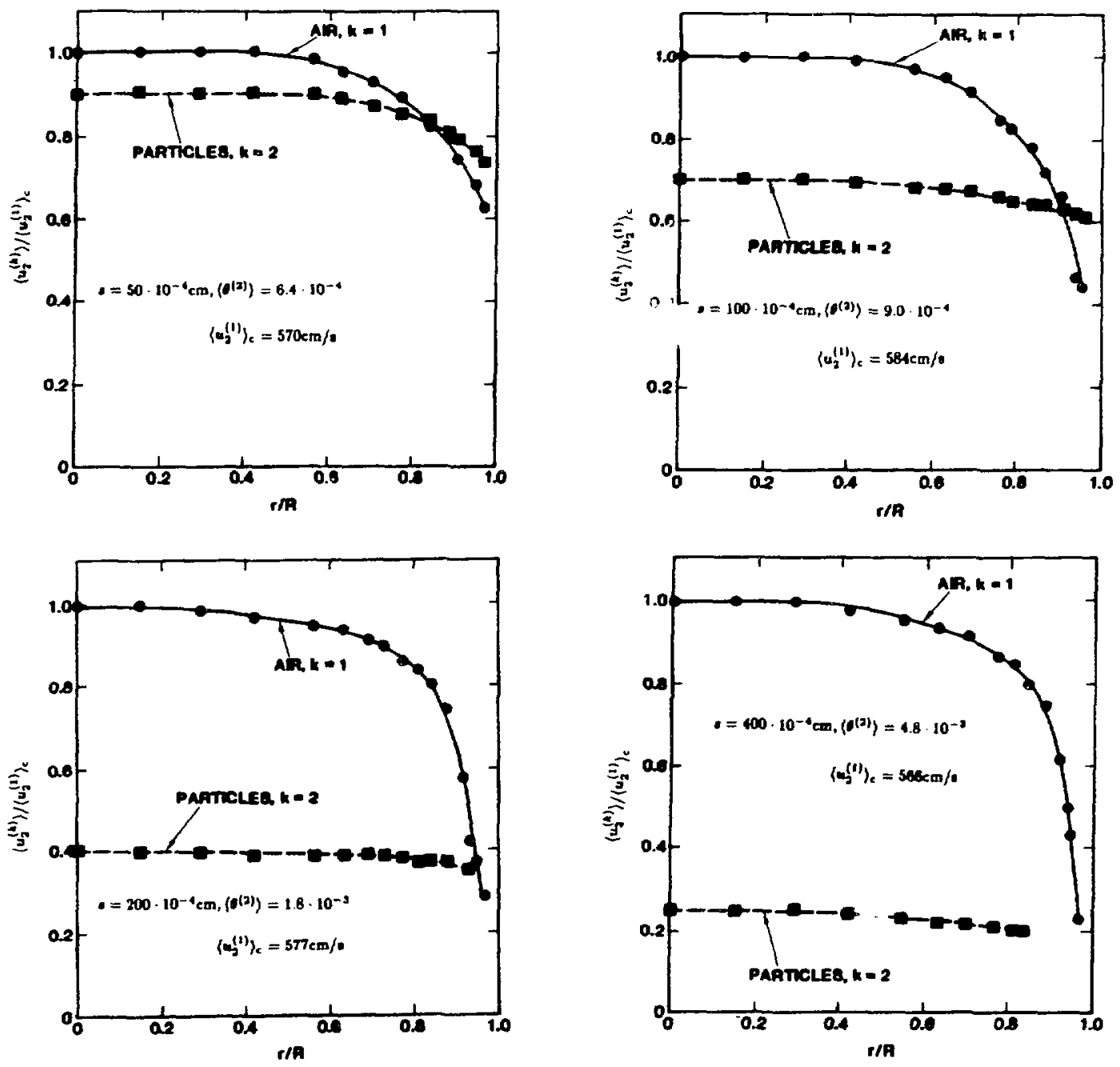

Figure 16. Velocity profile data from the pneumatic transport experiments of Lee and Durst (1982). The pipe radius $R$ is $2.09 \mathrm{~cm}$.

There are two important features of this velocity profile data that have not 
yet been explained. The first feature is seen in the cases $s=50 \cdot 10^{-4} \mathrm{~cm}$ and $s=100 \cdot 10^{-4} \mathrm{~cm}$, where the mean velocity of the particles is larger than that of the air near the pipe wall. The second feature is prominent in only the case $s=400 \cdot 10^{-4} \mathrm{~cm}$ where the apparent drag force is much larger than the force on a single sphere at that relative Reynolds number.

Clearly the upward velocity of the air must be larger than that of the particles over most of the pipe in order for the particles to be lifted against the downward force of gravity. This is because the only other possible source of upward acceleration of the particles is the pressure gradient, the action of which is negligible on the solid material due to the very small $\left\langle\theta^{(2)}\right\rangle$. (The Lee and Durst problem falls into the class of what is called a "dusty gas".)

The physical explanation for the particle velocity exceeding the air velocity near the wall is that of momentum diffusion by the turbulent fluctuations in particle velocity. Evidently the turbulence in the particle field is sufficiently large that the radial transport of axial particle momentum occurs at a rate great enough to enable the particles to lift the fluid near the wall. This results in a reversal in the direction of momentum exchange between the two materials.

To understand the second inexplained feature of the Lee and Durst experiments, a closer examination of the data is required. Let $u_{c}$ be the relative mean velocity at the pipe center, and consider the relative Reynolds number based on $u_{c}$. Figure 14 provides a value of the drag coefficient for a single sphere at that relative Reynolds number. This can be used to compute the single-sphere free-fall velocity $V_{0}$ using the definition of the drag coefficient. The downward force per unit sphere volume due to gravity is $\rho^{(2)} g$ which is balanced by the force in Eq. (6.1), with $u$ replaced by $V_{0}$. Hence

$$
V_{0}=\left(\frac{8}{3} \frac{\rho^{(2)} g s}{\rho^{(1)} C_{d}}\right)
$$

Table 2 is a list of values of $u_{c}$ and $V_{0}$. In Table $2, R e=2 s u_{c} / \nu^{(1)}, \nu^{(1)}=$ $0.145 \mathrm{~cm}^{2} / \mathrm{s}, \rho^{(1)}=1.225 \cdot 10^{-3} \mathrm{~g} / \mathrm{cm}^{3}, \rho^{(2)}=2.7 \mathrm{~g} / \mathrm{cm}^{3}$, and $\Delta=\left(u_{c}-V_{0}\right) / u_{c}$.

Table 2. Comparison of the single-sphere free-fall velocity with the data of Lee and Durst (1982).

\begin{tabular}{lllllll}
\hline $\begin{array}{l}s \cdot 10^{4} \\
(\mathrm{~cm})\end{array}$ & $\left\langle\theta^{(2)}\right\rangle$ & $\begin{array}{l}u_{c} \\
(\mathrm{~cm} / \mathrm{s})\end{array}$ & $R e$ & $C_{d}$ & $\begin{array}{l}V_{0} \\
(\mathrm{~cm} / \mathrm{s})\end{array}$ & $\Delta$ \\
\hline 50 & $6.4 \cdot 10^{-4}$ & 57 & 3.9 & 8.0 & 61 & -0.07 \\
100 & $9.0 \cdot 10^{-4}$ & 175 & 24 & 2.4 & 153 & +0.13 \\
200 & $1.8 \cdot 10^{-3}$ & 346 & 95 & 1.1 & 327 & +0.05 \\
400 & $4.8 \cdot 10^{-3}$ & 425 & 230 & 0.76 & 556 & -0.31
\end{tabular}

Notice that in the case $s=400 \cdot 10^{-4} \mathrm{~cm}$, the experimental relative mean velocity $u_{c}$ is much smaller than the single-sphere free-fall velocity $V_{0}$. This means that 
an effect is at play that tends to increase the drag in that case. According to the results of the previous section, the level of $\left\langle\theta^{(2)}\right\rangle$ in the Lee and Durst experiments is much too small for the long-range part of the exchange force to be significant. It is well known, however, that a flow with a pre-existing level of turbulence will produce a larger drag force on a single fixed sphere than a nonturbulent uniform flow (Clift, Grace and Weber 1978, 268). Therefore, the indication is that the production of turbulence energy in the boundary layer, with subsequent diffusion toward the pipe center, is responsible for enhancing the short-range part of the drag force in this case.

These two facets of the pnuematic transport data of Lee and Durst present a significant challenge for the multimaterial $k-\epsilon$ theory. This is because the new theory contains two entirely different sources of turbulence energy and decay rate. One source is the usual source due to gradients in the mean flow. The other is the creation of turbulence energy due to mean interpenetration of the two materials. These sources must act in the proper proportion with respect to one another in order to produce the correct turbulent viscosity of each material. (The exchange of turbulence energy and decay rate between materials provides an additional complexity that is extremely difficult to deal with on physical grounds. For that reason, energy exchange effects are neglected in the present work.) Because the turbulent viscosity determines the velocity profile and also contributes to the drag force, the momentum equations and turbulence equations are coupled together intimately. Hence this pneumatic transport problem represents a severe test of the universality of the multimaterial $k-\epsilon$ formulation proposed here.

For an initial comparison, consider the special case in which the volume fraction of the particles is uniform across the entire radius of the pipe. In this approximation, the particle-free zone near the pipe wall is neglected. This enables solution of the conservation equations using the two-dimensional FORTRAN computer code described in Chapter V, for this one-dimensional problem. The calculation proceeds in a manner identical to the turbulent channel flow problem solved in Chapter V: arbitrary initial velocity profiles are allowed to develop in time to a steady state as the processes of creation, destruction and diffusion come into balance.

The pressure gradient required to support the steady flow can be estimated from the experimental data in Fig. 16. A simple force balance shows that the pressure gradient $\Delta p$ is

$$
\Delta p=\frac{4}{D} \tau_{w}+[\rho] g
$$

where $\tau_{w}$ is the viscous shear stress at the pipe wall and $[\rho] g$ is the weight of the fluid mixture. (This neglects any momentum loss due to particle-wall interactions.) Since the wall stress $\tau_{w}$ can only be obtained approximately from the data and the particle-wall interactions are neglected in (6.22), the actual $\Delta p$ used in the calculations was adjusted slightly so that the computed centerline velocity was 
about equal to the measured value. The $\Delta p$ from (6.22) and that which was used in the calculations are given in Table 3.

It is of interest to compute the effective pipe friction factor $f$, defined by

$$
\Delta p=\frac{1}{2} \rho u_{c}^{2} \frac{f}{D} \pm[\rho] g
$$

where $u_{c}$ is the air centerline velocity. Here the plus sign is taken for upward pipe flow and the minus for downward flow. This too is listed in Table 3, where $f$ is based on the $\Delta p$ actually used in the computations.

Table 3. Pressure gradient and friction factor for the pnuematic transport

\begin{tabular}{|c|c|c|c|}
\hline $\begin{array}{l}\overline{s \cdot 10} \\
(\mathrm{~cm})\end{array}$ & & $\Delta p \quad$ (used) & $\bar{f}$ \\
\hline 50 & 4.05 & 4.0 & 0.023 \\
\hline 100 & 3.88 & 4.3 & 0.022 \\
\hline 200 & 6.39 & 7.0 & 0.72 \\
\hline 400 & 14.5 & 14.7 & 0.017 \\
\hline
\end{tabular}

Notice that the pipe friction factor falls into the range of that which would be expected for pure fluid at this pipe Reynolds number (Baumeister and Marks 1967 ed., 3-60).

Comparison of the computations to the experimental data provides additional confidence in the multimaterial $k-\epsilon$ theory, while at the same time some questions are raised, as discussed next. Figure 17 compares one set of calculations (designated a) to the Lee and Durst data. There it can be seen that the main features of the experimental velocity profiles are represented fairly well. Note that the particle velocity exceeds the gas velocity near the wall in the first two cases. This is a consequence of diffusion of the particle momentum by fluctuations in the particle velocity. Note too that the computed relative velocity between the materials is approximately correct near the center of the pipe, but deviates from the data near the wall. The predicted relative velocity in the case $s=400 \cdot 10^{-4} \mathrm{~cm}$ is about $496 \mathrm{~cm} / \mathrm{s}$ which is to be compared with $556 \mathrm{~cm} / \mathrm{s}$ for a single sphere (see Table 2). This means that the turbulence generated in the boundary layer has the effect of providing a pre-existing nonuniformity in the flow causing a significant increase in the drag on the particles.

One of the main questions that Fig. 17 raises has to do with the velocity boundary condition on the particulate field. In the Fig. 17 calculation, the particle field is subjected to a no-slip velocity condition at the pipe wall. This is not exactly correct for two reasons. The first is that a nearly particle-free region is observed to exist close to the wall. The second reason is that any particles that do manage to impact the wall can be expected to reflect with little or no loss in kinetic energy. This indicates that the wall should be well represented by a reflective boundary 

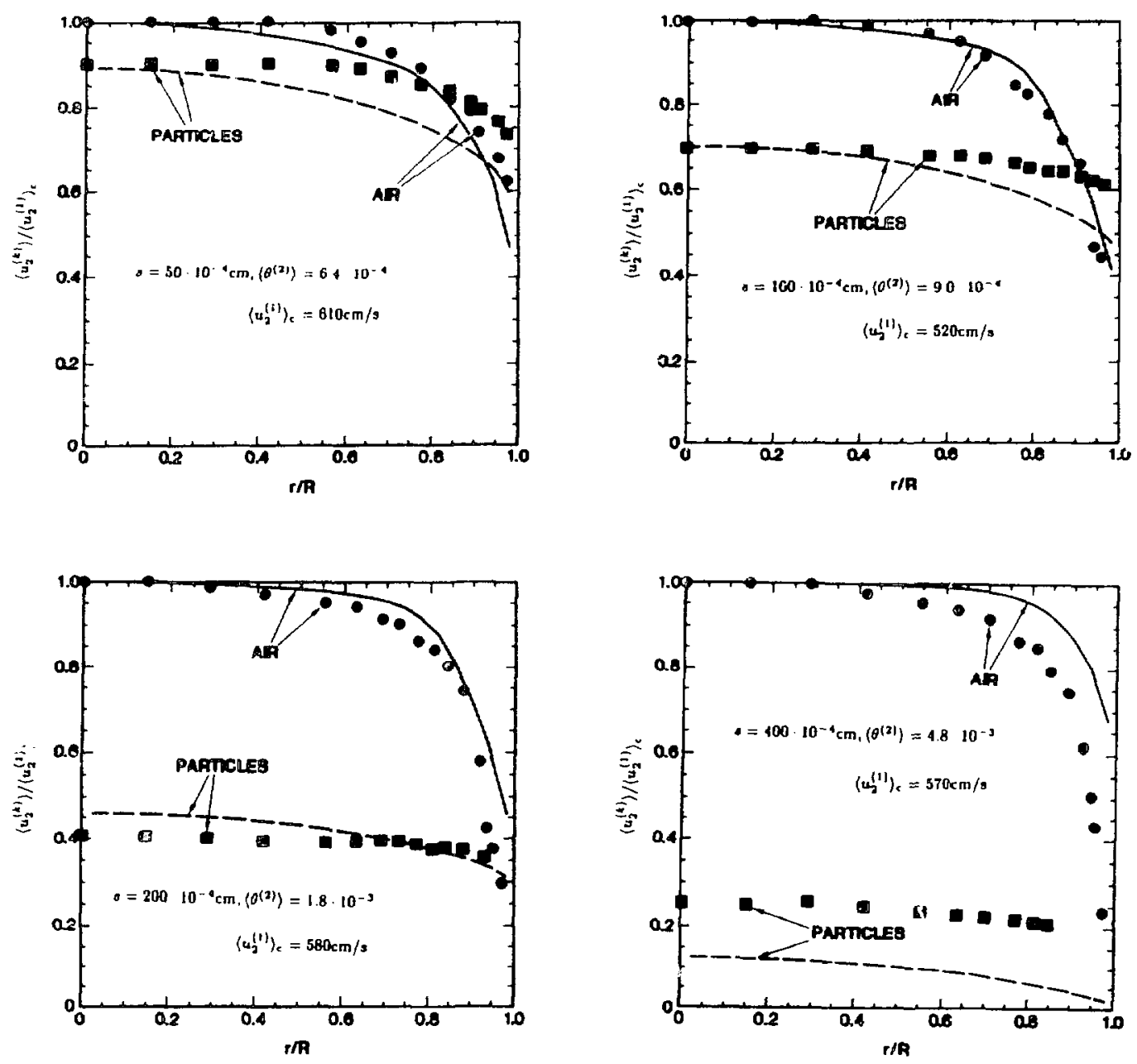

Figure 17. Comparison of velocity profiles form the multimaterial $k-\epsilon$ theory with the pneumatic transport data of Lee and Durst (1982).

for the particles. (There is of course no question that the wall is no-slip with regard to the fluid velocity.) Unfortunately this is not exactly the case, at least within the current construct of the theory. This fact is evident in the next set of comparisons.

Figure 18 is a comparison of calculation a, shown in Fig. 17, to a second computation (calculation b) which is in all respects identical except for a freeslip boundary condition on the particles at the wall. The comparison is just as might be expected. The particle velocity increases near the wall with the free-slip condition. This means the air velocity can also increase near the wall (to keep the relative velocity about the same). The effect is much greater for the small particle case than the large particle case. In the small particle case, both the solid 

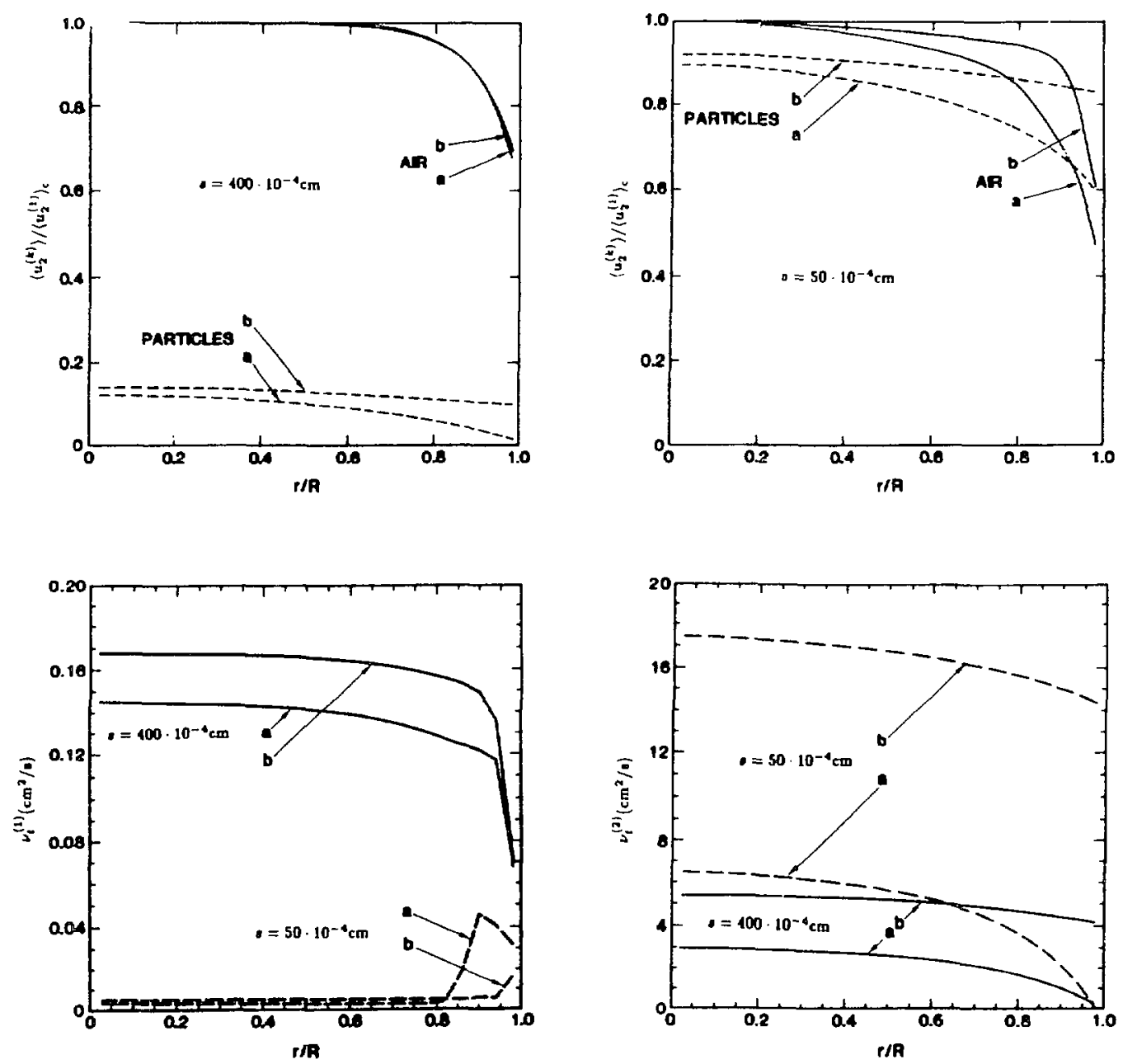

Figure 18. Comparison of no-slip (a) and free-slip (b) boundary conditions on the particle field.

velocity and the air velocity exceed the measured velocities in the near wall region by a substantial amount in calculation $b$. Whereas the particle velocity profile in calculation a is closer to the data, it is still not quite right.

Evidently the particle velocity boundary condition lies somewhere between no-slip and free-slip. One could improve on the fundamental theory by making use of some knowledge of the inelasticity (friction) in the particle-wall collision process. In the present work however, boundary effects have not been the principal focus of attention.

The main attention here has been the matter of fluctuational energy production by interpenetrating motion. The creation of fluctuational energy by interpenetration adds to the turbulence created by mean flow gradients. The magnitude of 

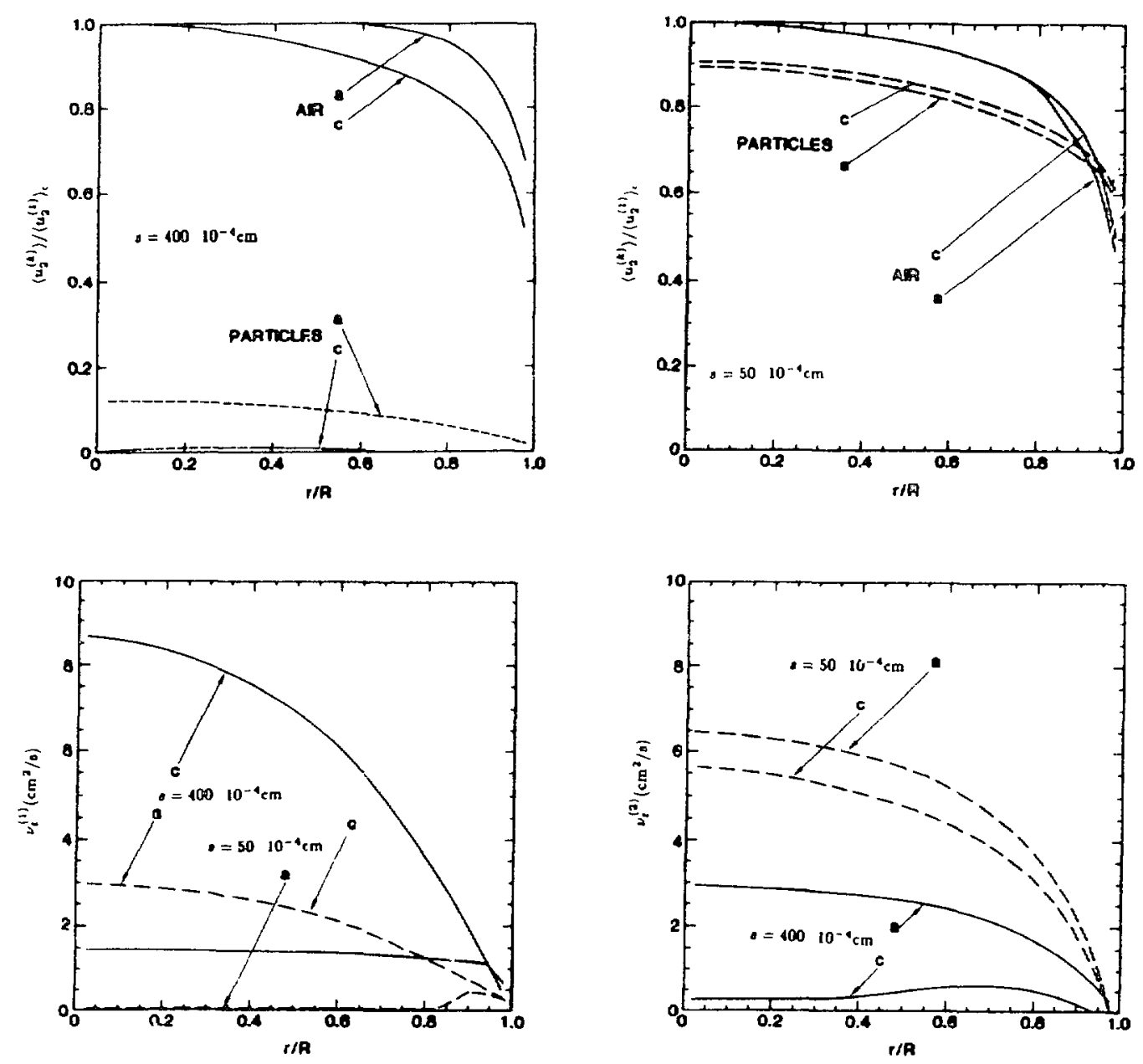

Figure 19. Comparison of a calculation including the interpenetrational creation term (a) to the same computation omitting the creation due to interpenetration (c).

this multimaterial effect is shown in Fig. 19. There a third calculation (c), in which the interpenetrational creation term is omitted, is compared to calculation a. In this comparison, the largest effect occurs in the large particle case, where the level of turbulence intensity is lowered enough to reduce the drag to where the particles remain barely suspended by the air. (Note that the decrease in intensity is accompanied by a diminished decay rate enabling the turbulent viscosity for the air to increase.)

The comparison of calculations $c$ and a illustrates a behavior of the theory that suggests an area in which the model can be improved. Notice that in the small particle case, there is very little difference in the velocity profiles when the 
interpenetrational creation term is omitted. However, there is a large difference in the turbulence viscosity for the air. The effect of the interpenetrational creation term is to reduce $\nu_{t}^{(1)}$ to a very small level in the central portion of the pipe. This can only occur if the creation in dissipation rate occurs at a rate that is disproportionate with respect to the rate of creation in fluctuational energy, which is probably the case for the air. This evidence leads to the conclusion that the inverse time scale relating the energy creation rate to the rate of $\left\langle\epsilon^{(k)}\right\rangle$ production is too large for the air in this case. Since the equation for $\left\langle\epsilon^{(k)}\right\rangle$ is largely a postulate anyway, evidence of the foregoing type can be used to set forth progressively better postulates, until a satisfactory model is produced. Evolution of this sort should be the subject of future investigations.

Another area of investigation is the whole subject of exchange of turbulence energy, which has been largely neglected in the present work. There is considerable potential for the improvement of the results in this section by including the exchange effect in the model. To see this, consider the distribution in root mean square velocity fluctuation $q^{(k)}$, compared to the data of Lee and Durst, plotted in Fig. 20. Observe that the level of turbulence intensity in the particles is lower than the data and that of the air is higher, over most of the pipe radius. It can be readily speculated that if the direction of fluctuational energy exchange were to be from the air to the particulate field, the agreement in $q^{(k)}$ distribution would improve.

A variety of other applications exist for which the multimaterial $k-\epsilon$ theory may be of significant value, and by whose guidance the theory can be further evoived. Some of these matters will be discussed in the next chapter. 


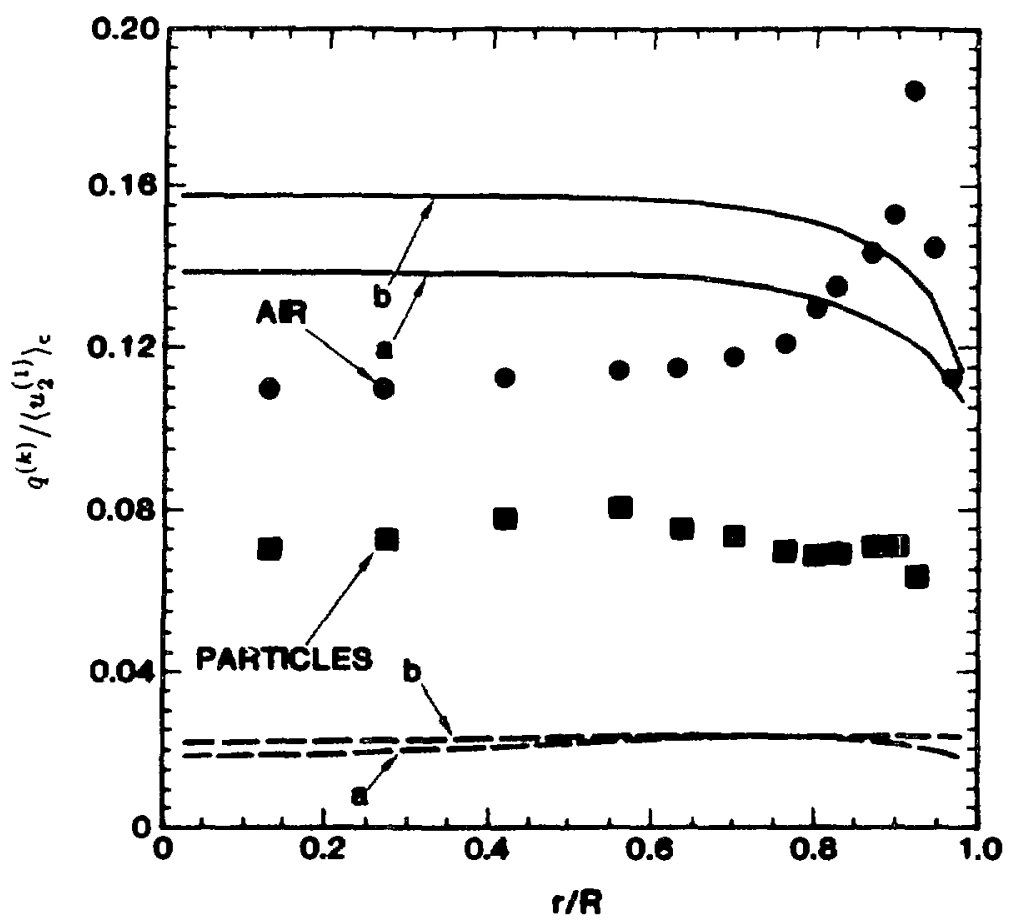

Figure 20. Distribution in root mean square velocity fluctuation for calculations $a$ and $b$, compared to the data of Lee and Durst (1982).

\section{References Cited}

Baumeister, T. and L. S. Marks, eds. 1967 ed. Standard handbook for mechanical engineers. New York: McGraw-Hill.

Clift, R., J. R. Grace and M. E. Weber. 1978. Bubbles, drops and particles. New York: Academic Press.

Lee, S. L. and F. Durst. 1982. On the motion of particles in turbulent duct flows. Int. J. Multiphase Flow. 8:125-146.

Richardson, J. F. and W. N. Zaki. 1954. Sedimentation and fluidization: Part I. Trans. Instn. Chem. Engrs. 32:35-53. 


\section{Chapter VII}

\section{Conclusions}

A great deal of ground has been covered in the preceding chapters, ranging from physical and theoretical considerations to numerical solutions and practical applications of the work. There is even a greater amount of further development that is required before many of the new ideas engendered here are brought to their full potential. The first section here contains a brief summary of the main results of the work and a description of how the results were obtained. The second section is a summary of the main conclusions about the work at can be drawn from the applications of the previous chapter. The final section is a potpourri of ideas concerning possible directions for the future evolution of the work.

\section{SUMMARY OF THE RESULTS}

There are two main physical phenomena associated with interpenetrating flows for which new results are obtained in this study. One is the diffusion of momentum and the other is momentum exchange. Developments in both areas are made by use of a mixture of physical reasoning with a particular statistical formalism (together with a bit of experimentation in the form of posing postulates for later verification).

The main theoretical tool that is utilized here is that of an ensemble averaging procedure for the derivation of the mean flow conservation equations. The new twist to this is the use of the "material functions" $\theta^{(k)}$. These are defined for each material such that if a point lies in material $k$ at an instant, then $\theta^{(k)}=1$, otherwise $\theta^{(k)}=\bigcup$ A natural consequence of this definition is the equation of motion satis. Sod by the material functions. Another consequen ce is that the ensemble average expectation of the material function $\left\langle\theta^{(k)}\right\rangle$ is related to the volume fraction of material $k$, defined in the continuum sense. This immediately gives a physical character to what otherwise would remain an abstract mathematical definition.

Having defined the material functions, the matter of deriving average (although unclosed) equations follows directly. In this, one must take care to define the velocity of material $k$ as the expected momentum per unit total expected mass. The result is a set of conservation equations for mass, momentum and fluctuational kinetic energy for each material. These equations bear a great resemblance to the Reynolds averaged Navier-Stokes equations. The main difference is that a material function appears inside the expectation sign in most terms, and a few additional terms arise that would be zero if only a single material were present.

This is the first important result of the work. In the momentum conservation equation, a multimaterial analog to the single-material Reynolds stress appears. It has exactly the same origin (both physically and mathematically), which suggests 
that an analogous closure scheme should be worthwhile. One additional term in the momentum equation clearly represents the effect of a conservative exchange of momentum between the interpenetrating materials. Close examination of the statistical nature of this exchange term reveals that the magnitude of the exchange force is partly dependent upon the intensity of the turbulence in each material.

The closure scheme used is a direct analog to the $k-\epsilon$ approach to singlematerial turbulence. The multimaterial Reynolds stress is represented by a turbulent viscosity, for each material. The turbulent viscosity $\nu_{t}^{(k)}$ is then approximated by the expression

$$
\nu_{t}^{(k)}=C_{\mu} \frac{\left\langle k^{(k)}\right\rangle^{2}}{\left\langle\theta^{(k)}\right\rangle\left\langle\epsilon^{(k)}\right\rangle}
$$

where $C_{\mu}$ is a universal constant (known from single-fluid theory), $\left\langle k^{(k)}\right\rangle$ is $\left\langle\theta^{(k)}\right\rangle$ times the fluctuational kinetic energy per unit mass in material $k$, and $\left\langle\epsilon^{(k)}\right\rangle$ is $\left\langle\theta^{(k)}\right\rangle$ times the turbulence energy decay rate per unit mass in material $k$. The use of (7.1) requires retaining evolution equations for $\left\langle k^{(k)}\right\rangle$ and $\left\langle\epsilon^{(k)}\right\rangle$ for each material. The model equations for $\left\langle k^{(k)}\right\rangle$ is designed by analogy to single-material theory, with two additional terms. One represents creation of turbulence energy by interpenetrating motion and the other represents the conservative exchange in $\left\langle k^{(k)}\right\rangle$. The additional terms are implied by the exact equations but their precise form is still uncertain. Hence it is only certain that they exist.

The use of similar evolution equations for $\left\langle\epsilon^{(k)}\right\rangle$ in each material depends upon the idea that dissipation of fluctuational energy occurs in each material, independent of the nature of the materials. For a mixture of two fluids, this concept is not difficult to comprehend because both materials can exhibit viscous dissipation at small length scales. For solid particles dispersed in a fluid, the idea of energy partitioning is needed in order to impart a physical interpretation to $\left\langle\epsilon^{(k)}\right\rangle$. That is, the occurrence of collisions between particles results in a transfer of translational kinetic energy into rotational energy, with an average loss in translational energy given by $\left\langle\epsilon^{(k)}\right\rangle$.

Hence the second main result of the study is closure of the average equations using a $k-\epsilon$ modeling methodology. An important part of this is the manner in which the turbulence quantities enter into the momentum exchange term. This is done in a way that is only suggested by the exact equations, and therefore is mostly obtained using physical reasoning. This combination of procedures produces a true tensorial character in the momentum exchange coefficient and introduces an analog to the thermal force in a mixture of molecular species. The magnitude of the exchange coefficient is found to depend upon three main physical effects. The first is viscous shear at interfaces, the second is flow separation and the third is multiple-entity effects.

Besides the theoretical formulation of the multimaterial conservation equations, a new method for the numerical solution of the equations has been developed 
during this study. This new method is motivated by defects in standard techniques that prohibit the accurate calculation of problems involving rapid spatial changes in the volume fraction. Since the mean velocity is defined by the momentum per unit total mass, computational techniques based on staggered control volumes can exhibit spurious velocities. This is because mass and momentum are conserved on spatially different control volumes, so that an interpolation is required to compute the velocity. When the momentum or mass varies rapidly in space, the interpolation can be inaccurate causing a poor representation of the velocity. This can be disastrous for multimaterial computations, which depend strongly on velocity differences.

This problem is remedied with the new method, which is based on a single control volume for mass and momentum, and in which a spurious velocity can never occur. The reason is that both total momentum and total mass approach zero at essentially the same rate so that the ratio is always well-defined. Since the scheme is based on coincident control volumes, all conserved quantities appear as cell-centered variables. The usual pressure-velocity decoupling that is experienced with such schemes is avoided by use of a MacCormack-like "predictor-corrector" formulation.

\section{CONCLUSIONS}

The first important conclusion is that a new formalism is developed for the derivation of average conservation equations for multimaterial flows. A variety of new results stem from the ensemble averaging technique used, with the generous help of physical reasoning. Some of the other main conclusions from the study are the following.

1. Representation of the multimaterial Reynolds stress by a $k-\epsilon$ formulation involving a turbulent viscosity times the mean rate of strain in each material has a great deal of merit. The good qualitative agreement in the velocity profile data in the pneumatic transport problem is the basis for this conclusion.

2. The essential parts of the momentum exchange force are contained in the modeled form of the momentum exchange function $K$, given in Eq. (6.20). All three applications in Chapter VI support this conclusion. Hence the effects of shear stress at material interfaces, drag due to flow separation and enhanced drag due to multiple-entity effecis are contained in (6.20).

3. The exchange in turbulence energy and decay rate, which was neglected in the Chapter VI problems, may have an important effect. This is supported by the last comparison of calculation and experimental data in Chapter VI, Sec. 3. 
4. The model equation for $\left\langle\epsilon^{(k)}\right\rangle$ can be improved by examining the behavior of the different forms of the inverse time scale multiplying the interpenetrational creation term. The calculations of Chapter VI, Sec. 3 indicate that a dependence on $\left\langle\theta^{(k)}\right\rangle$ may be required in this inverse time scale.

5. Additional physical effects such as a lift force due to gradients in the composite velocity and a force due to gradients in the fluctuational temperature are formulated but remain untested.

6. A new numerical solution procedure has been developed. This method enables accurate solution of the multimaterial conservation equations in multiple space dimensions.

\section{RECOMMENDATIONS FOR EXTENDED WORK}

The first most obvious need for further study is the application of the multimaterial $k-\epsilon$ theory to a wide variety of circumstances for which the general behavior is known. This will provide important heuristic guidance toward ways in which the theory can be improved, by understanding the strengths and weaknesses of the present formulation. During this evaluation process the untested portions of the theory should be carefully examined. Examples of applications that could provide useful information are the turbulent gas jet with solid particles, bubbly flow in a pipe at various volume fractions, the fluidized bed of fragmented solids and Poiseuille flow of a suspension. Experimental data exist for each of these problems.

Besides extended proof testing, a variety of specific areas of research that could provide useful extensions to this study are the following.

1. The matter of boundary effects was largely left unexamined in the prissent work. The whole subject of deriving relevant wall functions relating the wall stress to nearby mean flow quantities remains open for exploration.

2. The problem of deriving the correct volume fraction dependence of the interpenetrational inverse time scale appearing in the $\left\langle\epsilon^{(k)}\right\rangle$ equation remains unsettled.

3. Testing and improving on the formulation for energy and decay rate exchange between materials is also open for study.

4. In Chapter IV a number of two-point statistical correlations were defined. The formulation of the theory may benefit substantially from a careful re-visitation of these correlation functions. A small amount of good experimental data will go a long way in assisting the problem of closure in the momentum exchange and energy exchange terms that depend upon these two-point statistics. 
5. Careful measurement of the fluctuating quantities in sedimentation experiments would be very useful in the further development of the theory.

6. Finally, complicating physical processes such as phase change and chemical reaction are left as potential areas of exploration that could be studied within the statistical formalism utilized in this study. 


\section{Bibliography}

Amsden, A. A. and F. H. Harlow. 1978. K-TIF: A two-fluid computer code for downcomer flow dynamics. Los Alamos National Laboratory Report LA-6994.

Anderson, T. B. and R. Jackson. 1967. A fluid mechanical description of fluidized beds. Ind. and Eng. Chem. Fund. 6:527-639.

A ris, R. 1962. Vectors, tensors and the basic equations of fiuid mechanics. Englewood Cliffs: Prentice-Hall.

Batchelor, G. K. 1967. An introduction to fluid dynamics. Cambridge: Cambridge University Press.

Baumeister, T. and L. S. Marks, eds. 1967 ed. Standard handbook for mechanical engineers. New York: McGraw-Hill.

Bear, J. 1972. Dynamics of fluids in porous media. New York: American Elsevier.

Beran, M. J. 1968. Statistical continurm iheories. New York: Interscience Publishers.

Besnard, D. and F. H. Harlow. 1985. Turbulence in two-field incompressible flow. Los Alamos National Laboratory report LA-10187-MS.

Boothroyd, R. G. 1971. Flowing gas-solids suspensions. London: Chapman-Hall. Bradshaw, P. 1976. Turbulence. Berlin: Springer-Verlag.

Braginskii, S. I. 1965. Transport processes in a plasma. in M. A. Leontovich ed. Reviews of plasma physics, volume 1. New York: Consultants Bureau.

Burgers, J. M. 1969. Flow equations for composite gases. New York: Academic Press.

Buyevich, Y. A. 1971. Statistical hydromechanics of disperse systems. (3 parts). J. Fluid Mech. 49:489-507, 52:345-355, 56:313-336.

Chandrasekhar, S. 1943. Stochastic problems in physics and astronomy. Rev. Modern Phys. 15:1-89.

Clift, R., J. R. Grace and M. E. Weber. 1978. Bubbles, drops and particles. New York: Academic Press.

Cray Research, Inc. 1976. Cray-1 and Cray X-MP computer systems. Mendota Heights: Cray Research.

Crowe, C. T. 1982. Numerical models for dilute gas-particle flows. J. Fluids Eng. 104:297-303.

Crowe, C. T. and D. T. Pratt. 1972. Two-dimensional gas-particle flows. In R. B. Landis and G. J. Hordemann, eds. Proceedings of the 1972 heat transfer and fiuid mechanics institute. Stanford: Stanford University Press.

Currie, I. G. 1974. Fundamental mechanics of fiuids. Toronto: McGraw-Hill. 
Davis, R. W., E. F. Moore and C. T. Crowe. 1983. Numerical modeling of unsteady gas-particle flows around rectangles inside channels. in C. Taylor, ed. Numerical Methods in Laminar and Turbulent Flow. Swansea: Pineridge Press.

Drew, D. A. 1971. Averaged field equations for two-phase media. Studies in Applied Math. 50:133-166.

Dukowicz, J. K. 1980. A particle-fluid numerical model for liquid sprays. $J$. Comput. Phys. 35:229-253.

Elghobashi, S. E. and T. W. Abou-Arab. 1983. A two-equation turbulence model for two-phase flows. Phys. Fluids. 26:931-938.

Fan, L. T., S. H. Hwang, S. T. Chou and R. Nassar. 1985. Birth-death modeling of deep bed filtration: sectional analysis. Chem. Eng. Commun. 35:101-121.

Gawain, T. H. and J. N. Pritchett. 1970. A unified heuristic model of fluid turbulence. J. Comput. Phys. 5:383-405.

Hanjalić, K. and B. E. Launder. 1972. A Reynolds stress model of turbulence and its application to thin shear flows. J. Fluid Mech. 52:689-XXX.

Harlow, F. H. and A. A. Amsden. 1975. Numerical calculation of multiphase fluid flow. J. Comput. Phys. 17:19-52.

Harlow, F. H. and P. I. Nakayama. 1967. Turbulence transport equations. Phys. Fluids. 10:2323-2332.

- 1968. Transport of turbulence energy decay rate. Los Alamos Scientific Laboratory report LA-3854.

Harlow, F. H. and J. E. Welch. 1965. Numerical Calculation of time-dependent viscous incompressible flow with free surface. Phys. Fluids. 8:2182-2189.

Hetsroni, G. 1982. ed. Handbook of multiphase systems. New York: McGraw-Hill. Hinze, J. O. 1975. 2d ed. Turbulence. New York: McGraw-Hill.

Hirt, C. W. 1968. Heuristic stability theory for finite-difference equations. $J$. Comput. Phys. 2:339-355.

Hirt, C. W., A. A. Amsden and J. L. Cook. 1974. An arbitrary LagrangianEulerian computing method for all flow speeds. J. Comput. Phys. 14:227253.

Hirt, C. W., B. D. Nichols and N. C. Romero. 1975. Sola-A numerical solution alogrithm for transient fluid flows. Los Alamos National Laboratory Report LA-5852.

Ishii, M. 1975. Thermo-fluid dynamic theory of two-phase flow. Eyrolles: Direction des Études et Recherches d'Électricité de France.

Kashiwa, B. A. 1986a. A generalized MAC method for incompressible fuid fiow. Los Alamos National Laboratory Report LA-10853-MS. 
1986b. A predictor-corrector method for calculation of incompressible fluid flow dynamics. Los Alamos National Laboratory report LA-10854-MS.

Kays, W. M. 1966. Convective heat and mass transfer. New York: McGraw-Hill.

Laufer, J. 1951. Investigation of turbulent flow in a two-dimensional channel. National Advisory Committee for Aeronautics report 1053.

Launder, B. E. and D. B. Spalding. 1974. The numerical computation of turbulent flows. Comp. Meth. App. Mech. Eng. 3:269-289.

Lax, P. D. and B. Wendroff. 1964. Difference schemes for hyperbolic equations with high order of accuracy. Comm. on Pure and Appl. Math. 17:381-398.

Lee, S. L. 1982. Aspects of suspension shear flows. In Chia-Shun Yih, ed. Advances in applied mechanics. 22:1-65. New York: Academic Press.

Lee, S. L. and F. Durst. 1982. On the motion of particles in turbulent duct flows. Int. J. Multiphase Flow. 8:125-146.

Lourenco, L. M., M. L. Riethmuller and J. A. Essers. 1983. A kinetic approach to gas-solid suspensions. in J. T. Jurewicz, ed. gas-solid flows. New York: ASME.

Lundgren, T. S. 1967. Distribution functions in the statistical theory of turbulence. Phys. Fluids. 10:969-975.

- 1969. Model equation for nonhomogeneous turbulence. Phys. Fluids. 12:485-497.

. 1972. Slow flow through stationary random beds and suspensions of spheres. J. Fluid Mech. 51:273-299.

Maxey, M. R. and J. J. Riley. 1983. Equation of motion for a small rigid sphere in a nonuniform flow. Phys. Fluids. 26:883-889.

MacCormack, R. W. 1969. The effect of viscosity in hypervelocity impact cratering. AIAA Paper 69-354.

McTigue, D. F. 1982. A nonlinear consitutive model for granular materials: application to gravity flow. J. Applied Mech. 49:291-296.

Mih, W. C., C. K. Chen and J. F. Orsborn. 1971. Bibliography of solid-liquid transport in pipelines. Pullman: Albrook Hydraulic Laboratory.

Murray, J. D. 1965. On the mathematics of fluidization. Part 1. Fundamental equations and wave propagation. J. Fluid Mech. 21:465-493.

Needham, D. J. and .T. H. Merkin. 1983. The propagation of a voidage disturbance in a uniformly fluidized bed. J. Fluid Mech. 131:427-454.

Oates, G. C. 1974. Vector Analysis. in C. E. Pearson, ed. Handbook of applied mathematics. New York: van Nostrand Reinhold.

O'Rourke, P. J. 1981. Collective drop effects on vaporizing liquid sprays. Doctoral dissertation, Princeton University. Also, Los Alamos National Laboratory report LA-9069-T. 
Ratafia, M. 1973. Experimental investigation of Rayleigh-Taylor instability. Phys. Fluids. 16:1207-1210.

Reeks, M. W. 1983. The transport of discrete particles in inhomogeneous turbulence. J. Aerosol Sci. 14:729-739.

Reynolds, W. C. and T. Cebeci. 1976. Calculation of turbulent flows. in P. Bradshaw ed. Turbulence. Berlin: Springer-Verlag.

Richardson, J. F. and W. N. Zaki. 1954. Sedimentation and fluidization: Part I. Trans. Instn. Chem. Engrs. 32:35-53.

Roache, P. J. 1972. Computational fluid dynamics. Aiouquerque: Hermosa.

Rodi, W. 1980. Turbulence models and their application in hydraulics. The Netherlands: International Assoication for Hydraulic Research.

Schlichting, H. 1955. Boundary layer theory. J. Kestin translator. New York: McGraw-Hill.

Tennekes, H. and J. L. Lumley. 1972. A first course in turbulence. Cambridge: The MIT Press.

Townsend, A. A. 1976. 2d ed. The structure of turbulent shear flow. Cambridge: Cambridge University Press.

Travis, J. R., F. H. Harlow, and A. A. Amsden. 1976. Numerical calculation of two-phase flows. Nuc. Sci. Eng. 61:1-10.

Tsuji, Y. and Y. Morikawa. 1982. LDV measurements of an air-solid two-phase flow in a horizontal pipe. J. Fluid Mech. 120:385-409.

van Leer, B. 1977. Towards the ultimate conservative difference scheme. IV. A new approach to numerical convection. J. Comput. Phys. 23:276-299.

Vincenti, W. G. and C. H. Kruger. 1965. Introduction to physical gas dynamics. New York: John Wiley \& Sons.

Von Neumann, J. and R. D. Richtmyer. 1950. A method for the numerical calculation of hydrodynamic shocks. J. Applied Phys. 21:232-237.

Westbrook, C. K. 1977. Three-dimensional numerical modeling of liquid fuel sprays. The sixteenth symposium on combustion. Pittsburgh: The Combustion Institute.

Whitaker, S. 1973. The transport equations for multi-phase systems. Chem. Eng. Sci. 28:139-147.

Williams, F. A. 1985. 2d edition. Combustion theory. Menlo Park: BenjaminCummings.

Woodward, P. and P. Collela. 1984. The numerical simulation of two-dimensional fluid flow with strong shocks. J. Comput. Phys. 54:115-173.

Yakhot, V. and S. A. Orszag. 1986. Renormalization group analysis of turbulence. I. Basic theory. J. Sci. Computing. 1:3-51. 


\section{Appendix $\boldsymbol{A}$ \\ Numerical Calculation of Gradients}

This appendix describes the way in which numerical estimates of gradients in the mean flow quantities are computed. The basic scheme depends on the chain rule for differentiation. The technique makes use of the functional relation of physical coordinates to logical coordinates. For example, in logical $(i, j)$ space the position vector is

$$
\mathbf{x}=[x(i, j), y(i, j)]
$$

and some quantity $Q$ distributed in space is

$$
Q=Q(x, y)
$$

such that

$$
\left(\begin{array}{ll}
\frac{\partial x}{\partial i} & \frac{\partial y}{\partial i} \\
\frac{\partial x}{\partial j} & \frac{\partial y}{\partial j}
\end{array}\right)\left(\begin{array}{l}
\frac{\partial Q}{\partial x} \\
\frac{\partial Q}{\partial y}
\end{array}\right)=\left(\begin{array}{l}
\frac{\partial Q}{\partial i} \\
\frac{\partial Q}{\partial j}
\end{array}\right) .
$$

The numerical gradient is simply the solution to these linear equations, with the differentials replaced by finite-difference estimates. Hence the horizontal component of the gradient is

$$
\frac{\partial Q}{\partial x} \sim \frac{1}{d}\left(\Delta_{i} Q \Delta_{j} y-\Delta_{i} y \Delta_{j} Q\right),
$$

and the vertical component of the gradient is

$$
\frac{\partial Q}{\partial y} \sim \frac{1}{d}\left(\Delta_{i} x \Delta_{j} Q-\Delta_{i} Q \Delta_{j} x\right)
$$

in which

$$
d=\left(\Delta_{i} x \Delta_{j} y-\Delta_{i} y \Delta_{j} x\right) .
$$

In these expressions, the difference operators are defined

$$
\Delta Q=Q_{f}-Q_{b}
$$

where $Q$ is the distributed quantity, and the subscripts $f$ and $b$ refer to cellcentered locations on the forward and backward sides of the point for which the gradients are computed. Figure (A1) indicates the location in logical space of the $Q$ and coordinate information that is used in the gradient calculation for the facecentered gradients and the cell-centered gradients. Recall that the face-centered gradients are used in the viscous stress tensor (and are designated by the I I brackets), in both the predictor and corrector steps. The cell-centered gradients (designated with (( )) brackets) enter only into the advection operator. 
Predictor

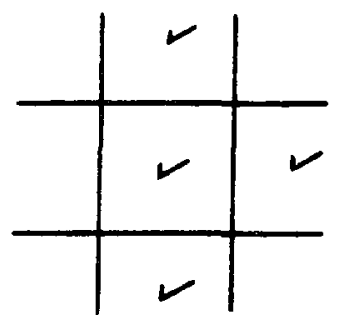

(a)
Corrector

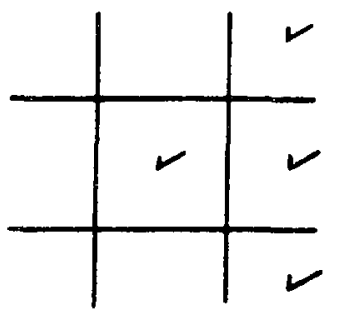

(b)
Advection

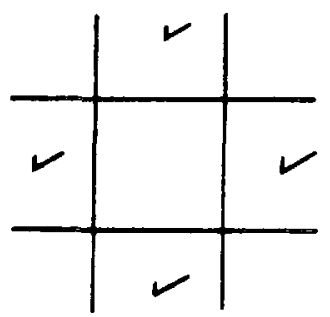

(c)

Figure A1. Location of data used in gradient calculations: a) data used in the gradients at one side in the predictor step, b) data used in the gradients at the same side in the corrector step, and c) data used in the cell-centered gradients used in both steps. 


\section{Appendix $B$}

\section{Relaxation Procedure}

This appendix provides a brief description of the point-wise relaxation procedure used to find the time advanced pressure and velocity in the predictorcorrector method. The basis of the scheme is that which was made popular by Hirt et.al. (1975).

The procedure is as follows. First, the explicit part of the change in momentum is computed, and saved, for each cell of the mesh. Then a test value of the new $\left\langle\theta^{(k)} u_{i}\right\rangle$ is obtained using an estimate of the pressure field. The new velocity is then derived, and estimates of the new $\left\langle\theta^{(k)}\right\rangle$ are obtained from the mass equations. This enables a residual $D$ to be determined by

$$
D=1-\sum_{k}\left\langle\theta^{(k)}\right\rangle
$$

This residual is then used to compute a change in pressure $\delta p$ that drives the residual toward zero in a point-wise Newton-Raphson fashion. The appropriate pressure change is

$$
\delta p=-\frac{D}{a^{2} \Delta t^{2}}\left[\frac{V^{2} \rho^{*}}{4\left(\mathbf{S}_{1} \cdot \mathbf{S}_{3}+\mathbf{S}_{2} \cdot \mathbf{S}_{4}\right)}\right] \Omega
$$

in each cell, where

$$
a= \begin{cases}1, & \text { predictor } \\ 1 / 2, & \text { corrector. }\end{cases}
$$

Here $\Omega$ is a relaxation coefficient such that $0<\Omega<1, S_{1}$ through $S_{4}$ are the outward normal surface vectors for the cell, corresponding to Fig. 7 , and the density is

$$
\rho^{*}=\left(\sum_{k} \frac{\left\langle\theta^{(k)}\right\rangle}{\rho^{(k)}}\right)^{-1} .
$$

At the beginning of the iteration, the predictor or corrector pressure from the previous time step can be used as the initial estimate of the pressure in each of the respective steps. The iteration process is repeated until the residual is of sufficiently small measure in all cells of the mesh. For a rectangular mesh, $\Omega=1.0$ is the maximum stable relaxation coefficient. As the nonorthogonality increases, $\Omega$ must be decreased to maintain a stable iteration. For example, a vaiue of $\Omega=0.8$ provides a stable relaxation for all of the problems shown in Chapter $\mathrm{V}$. 


\section{Appendix $C$ \\ Listing of the FORTRAN Computer Code}

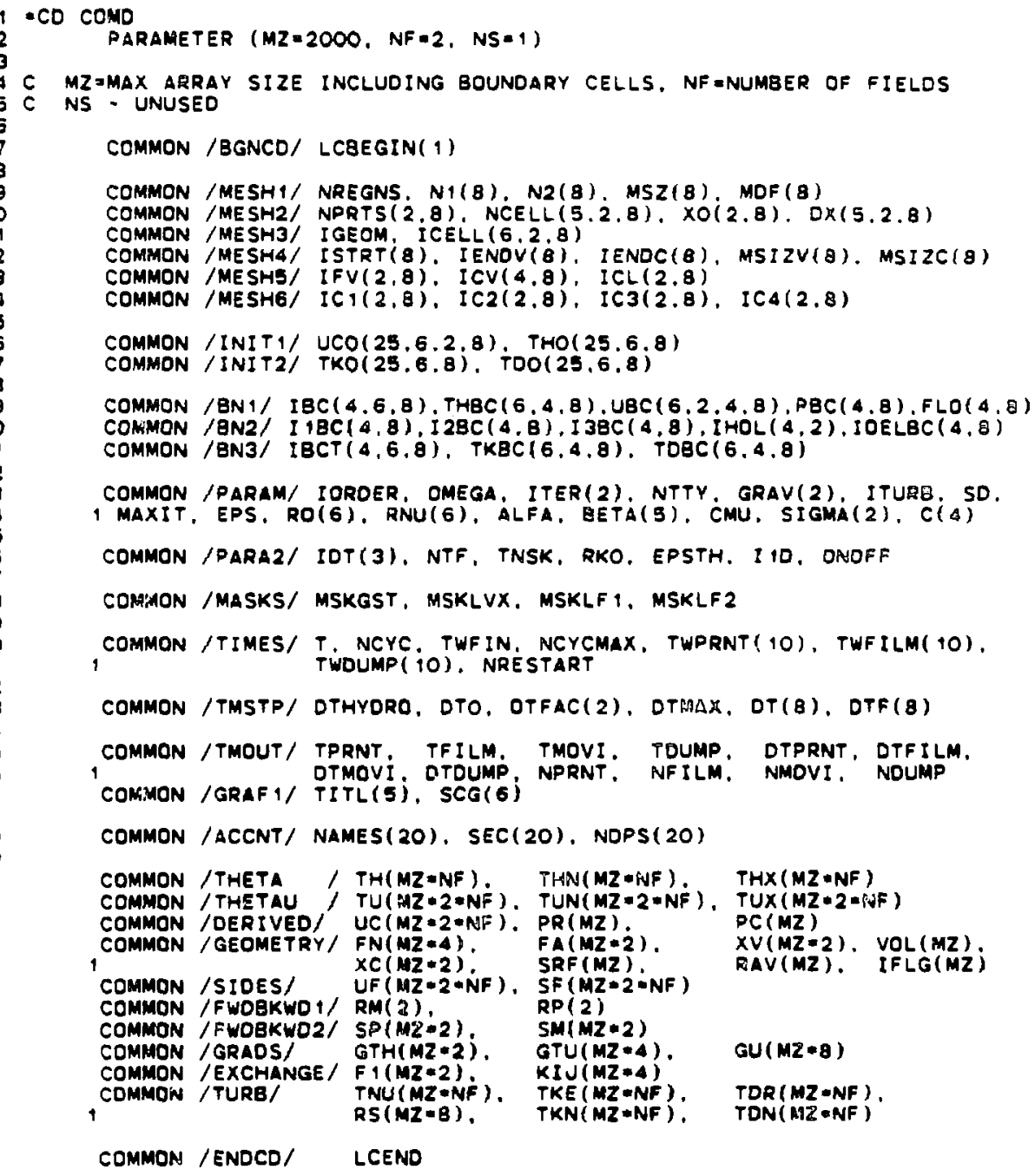




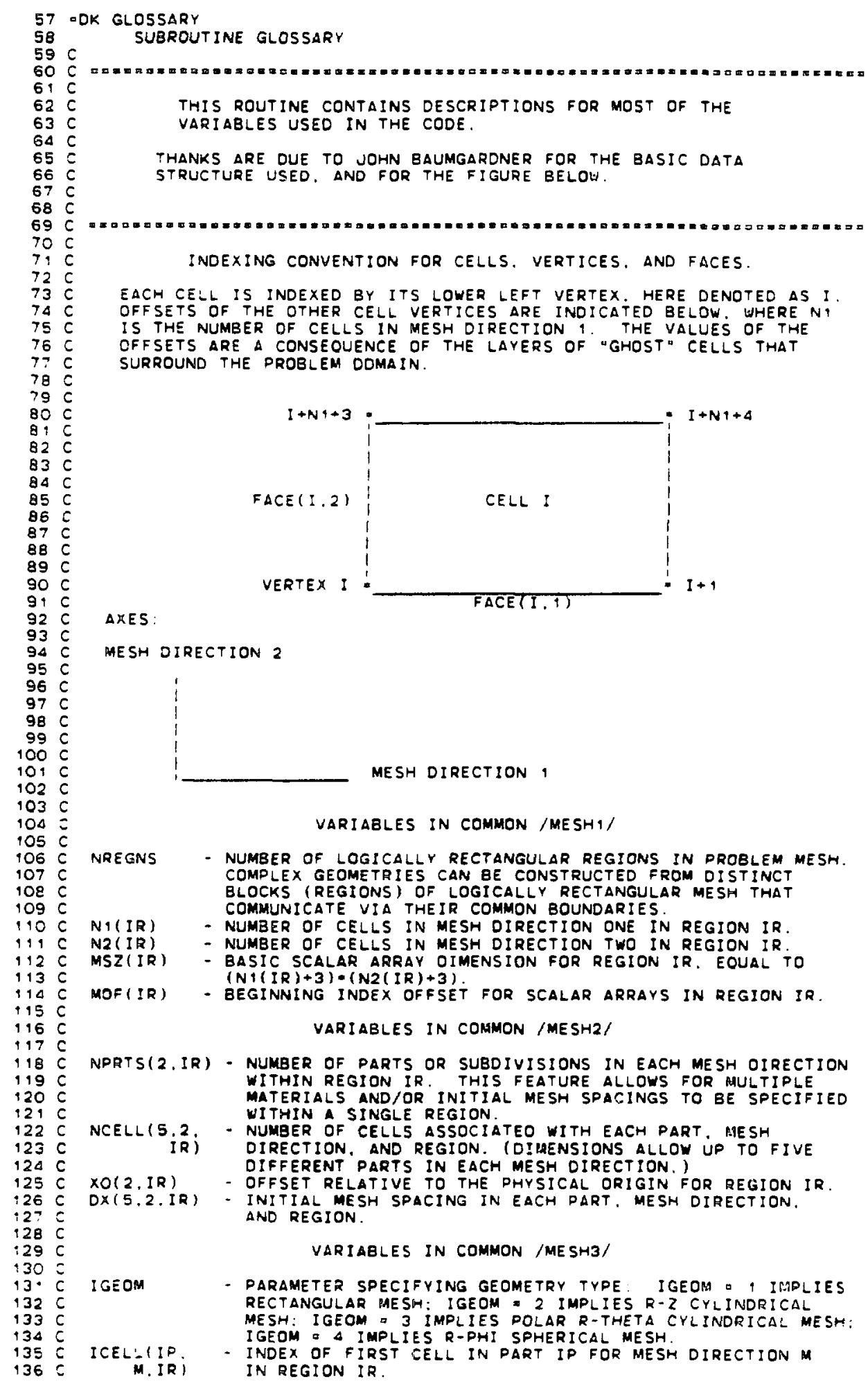




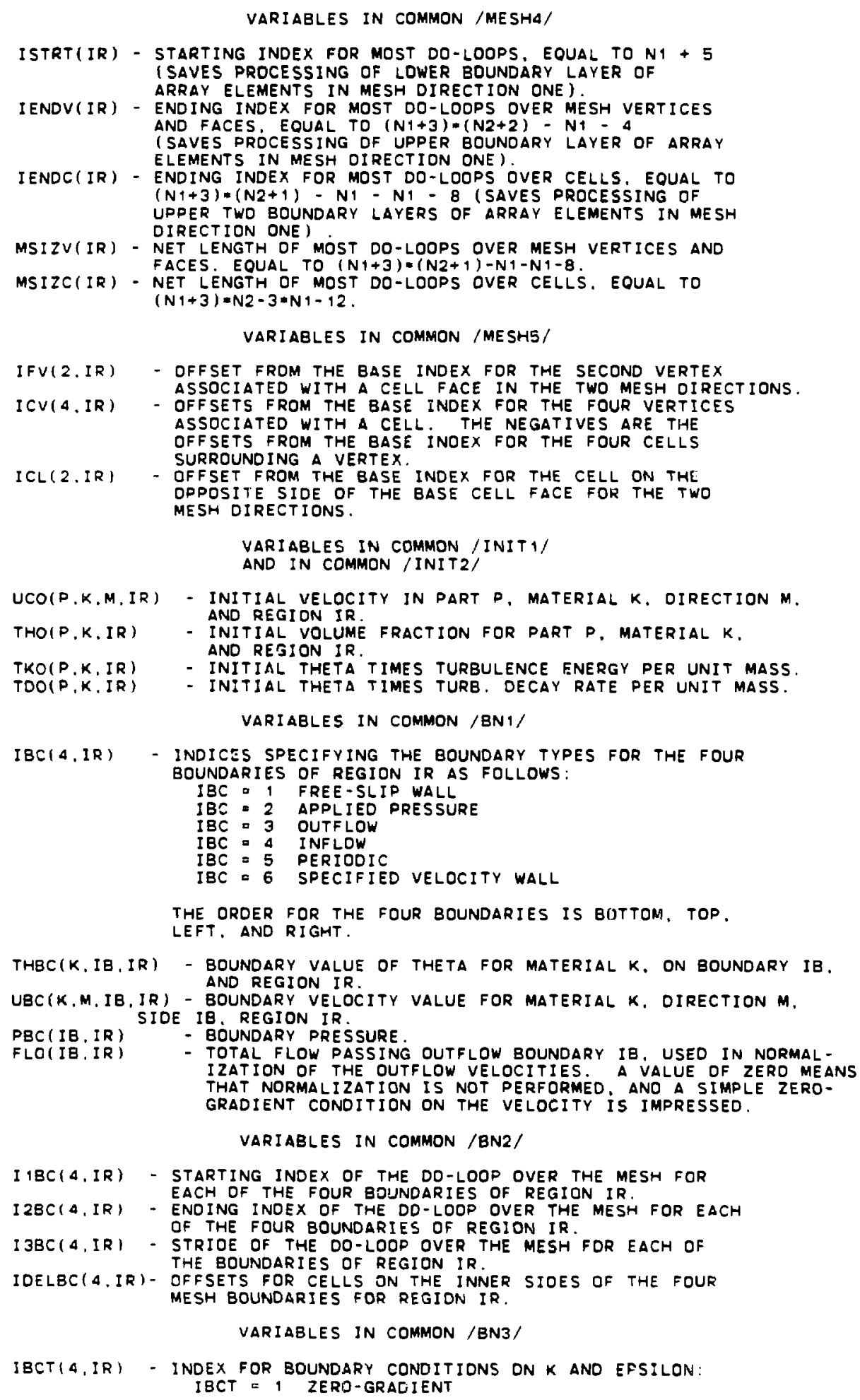




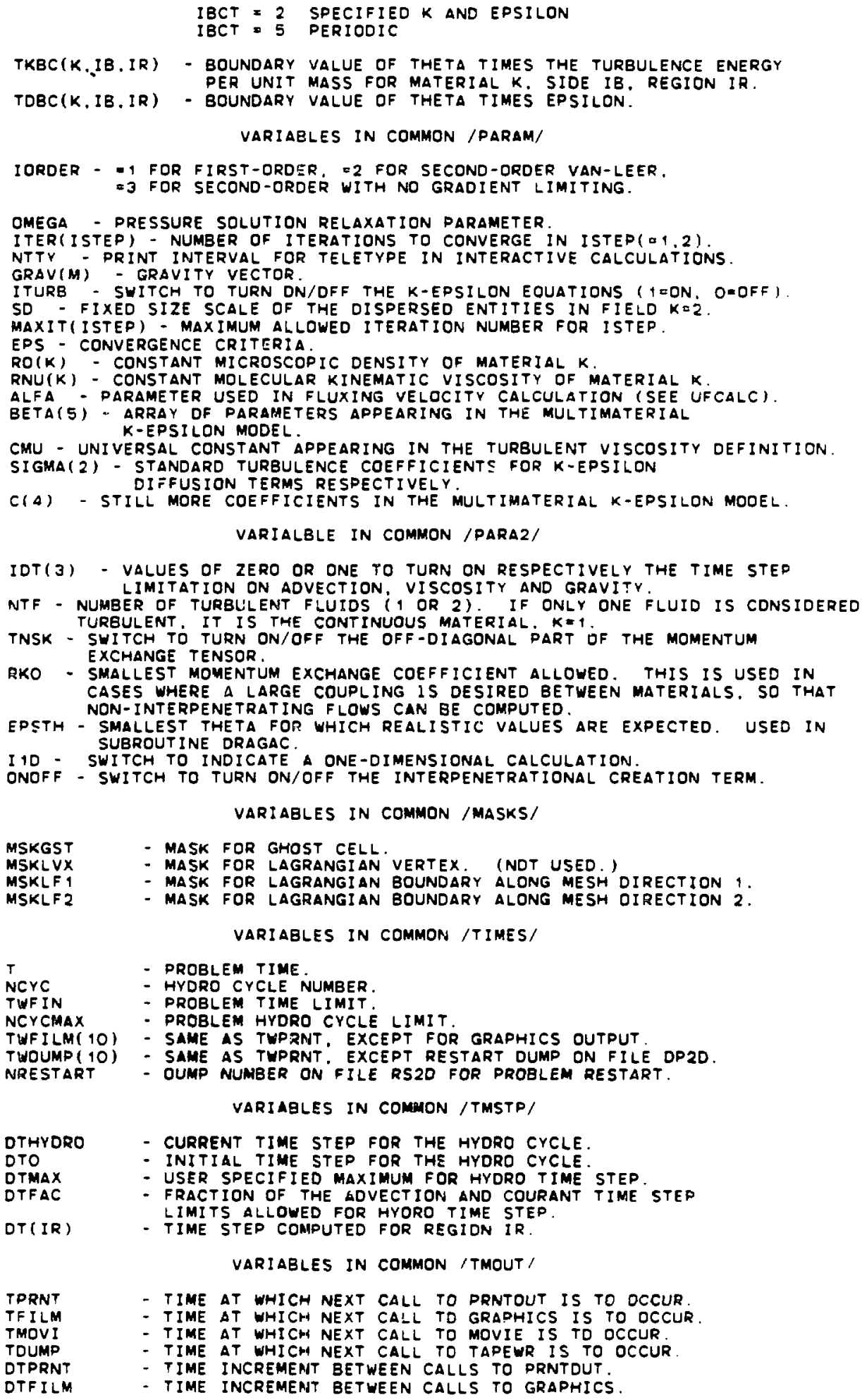




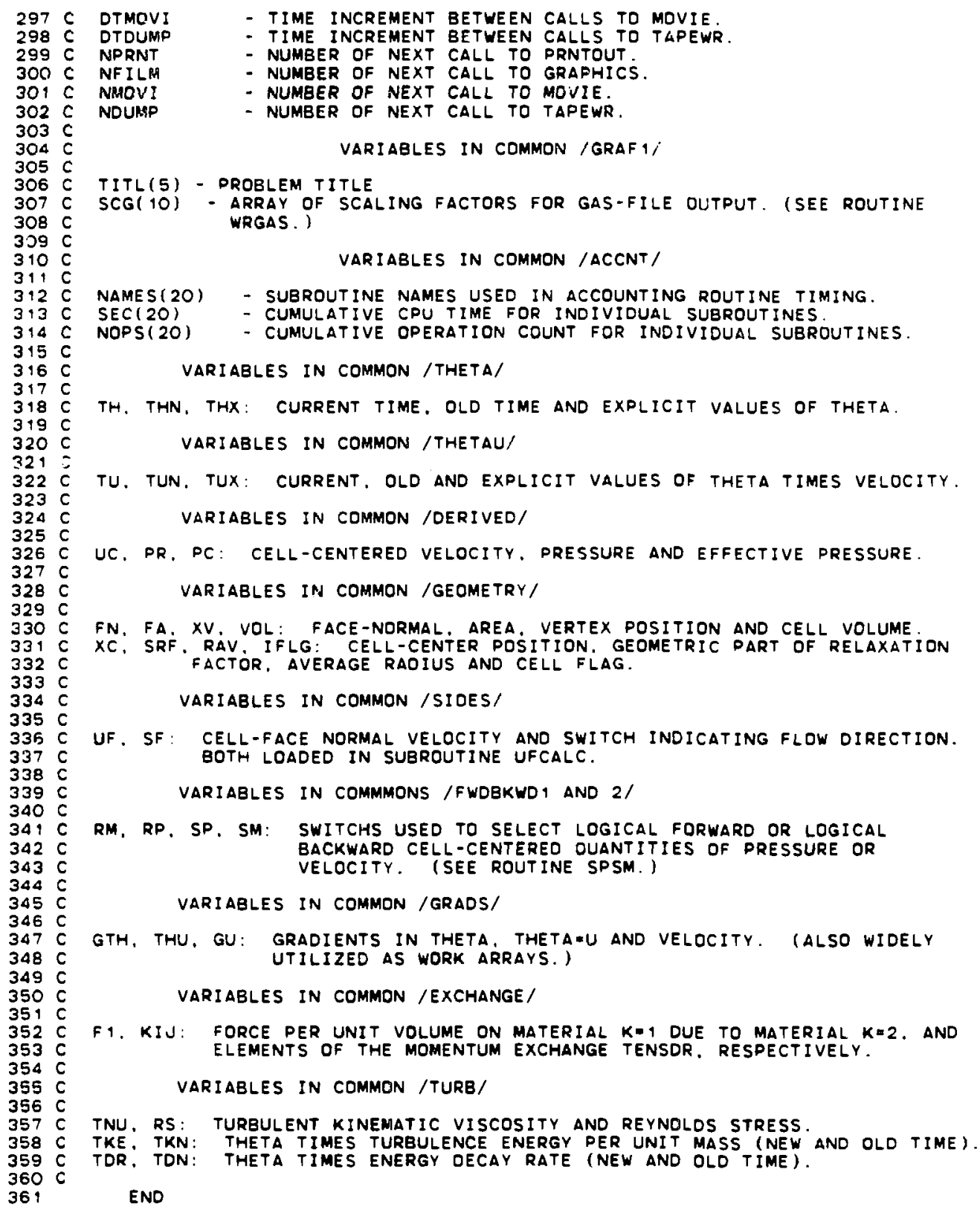




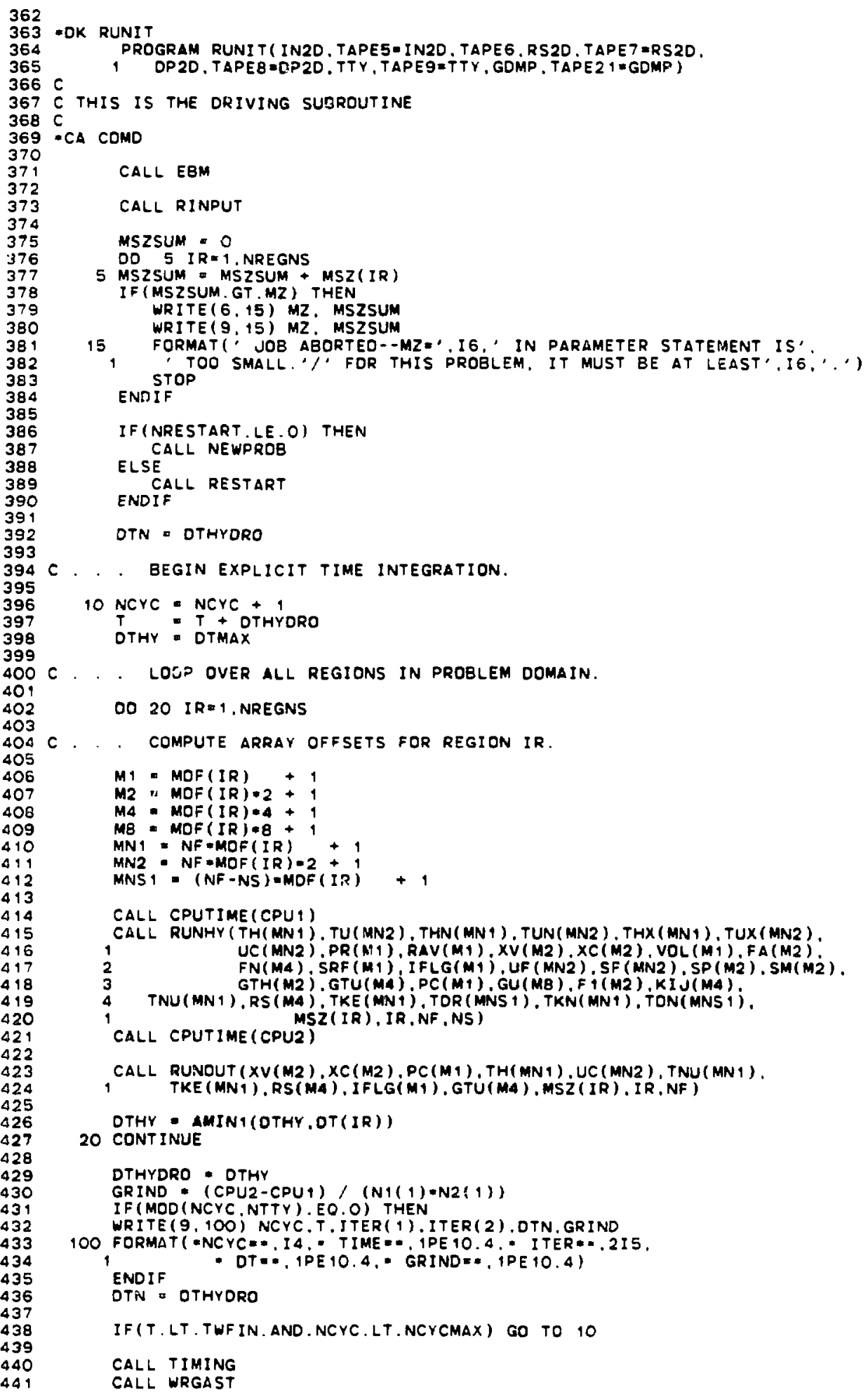




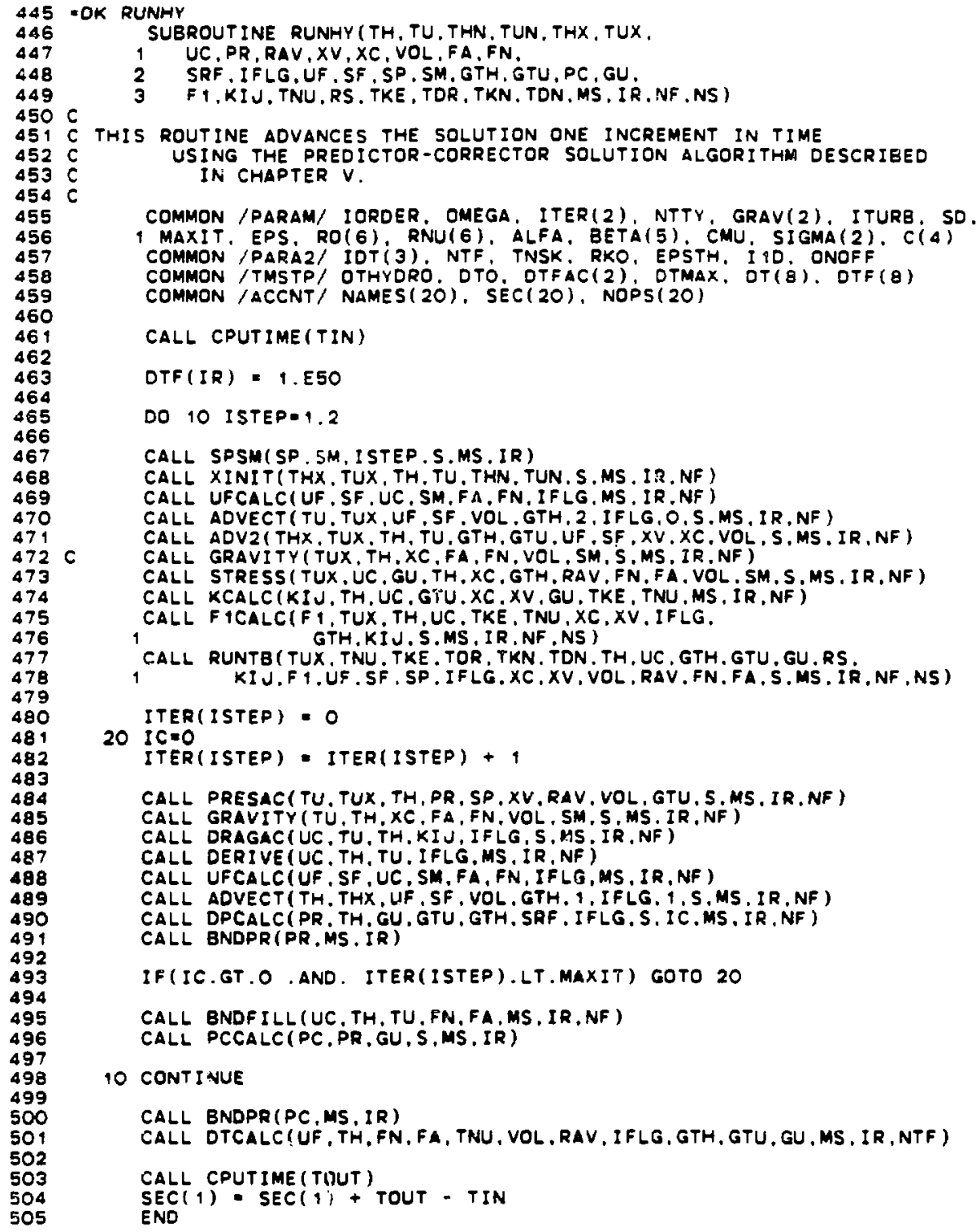




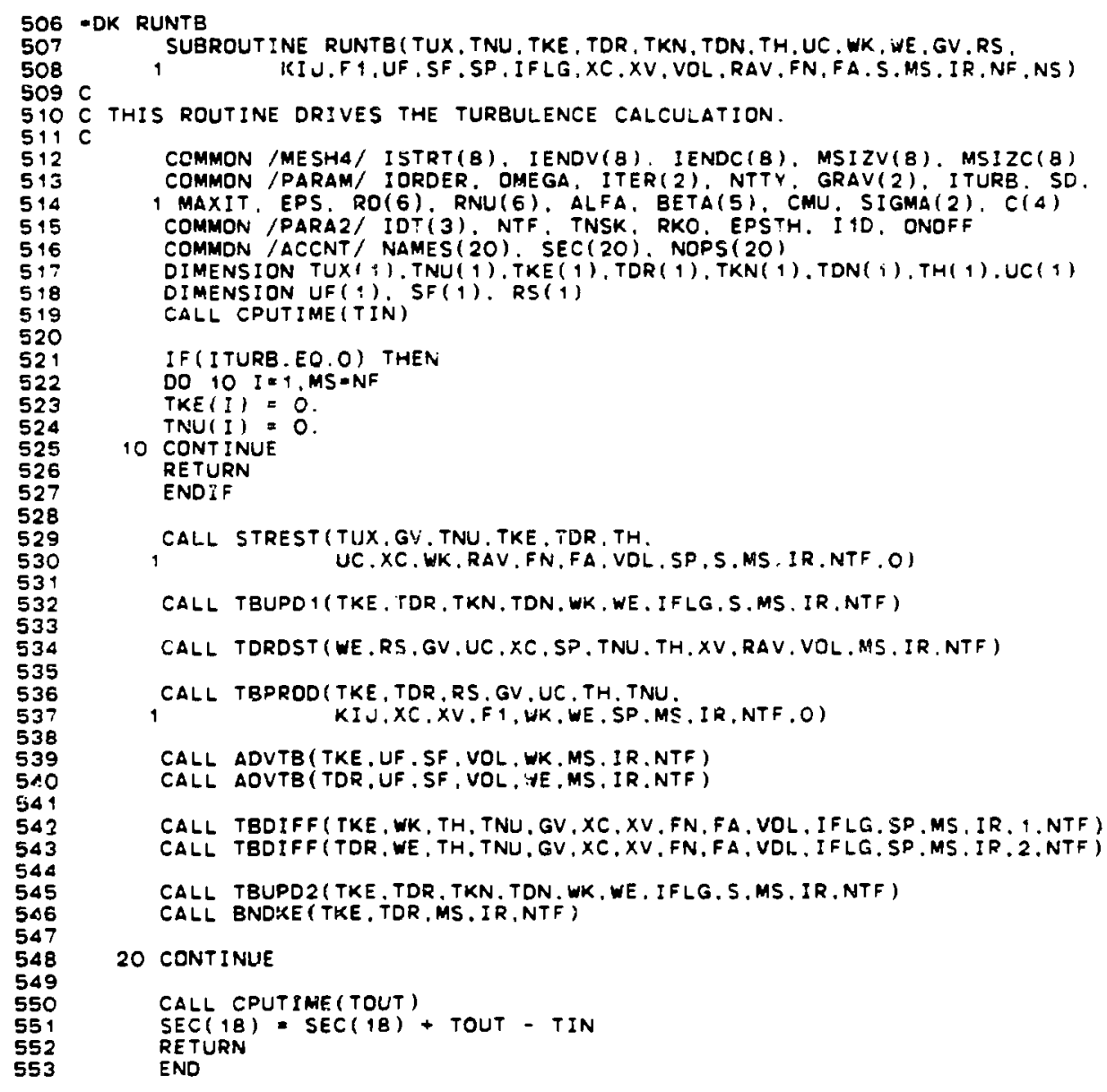




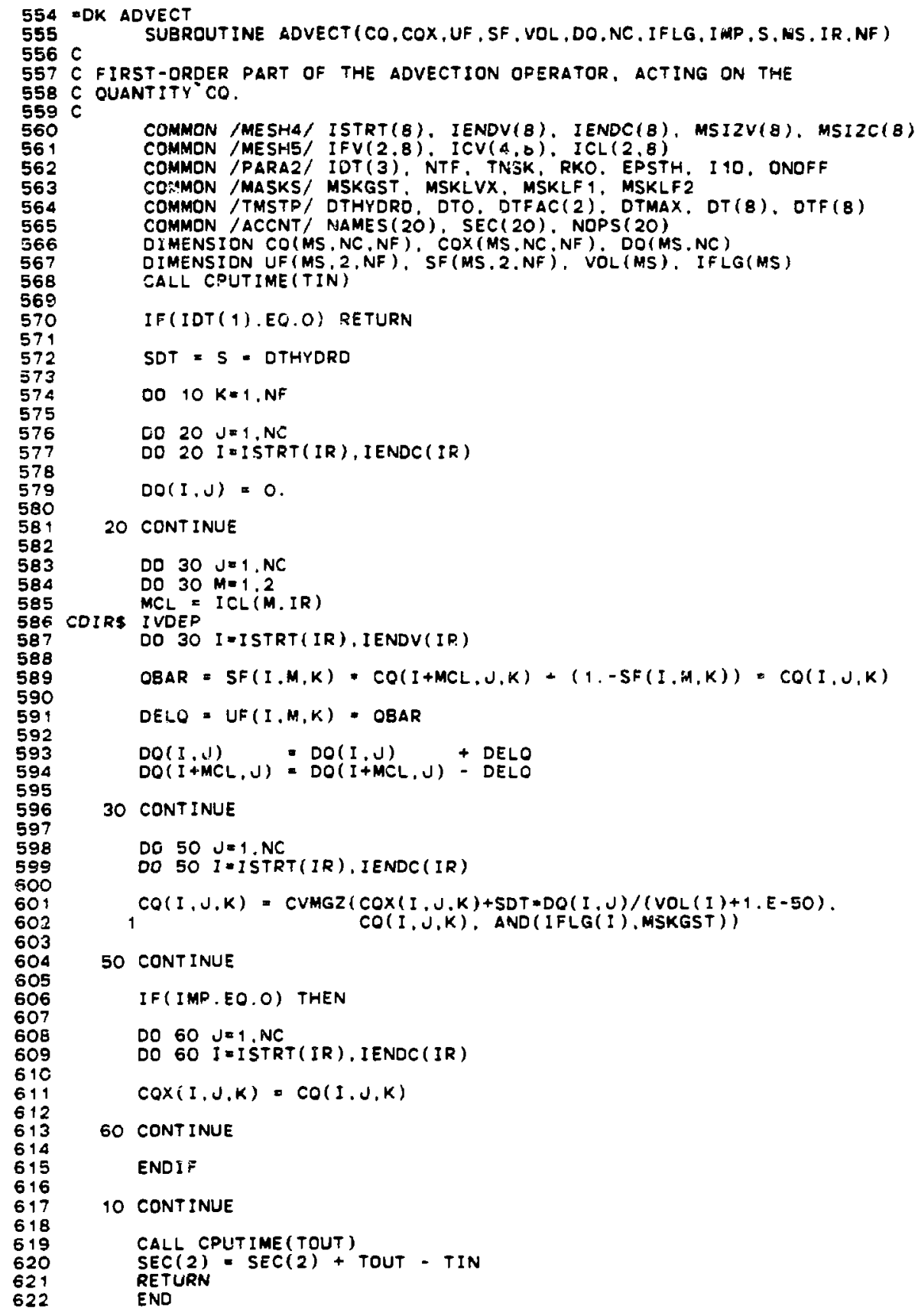




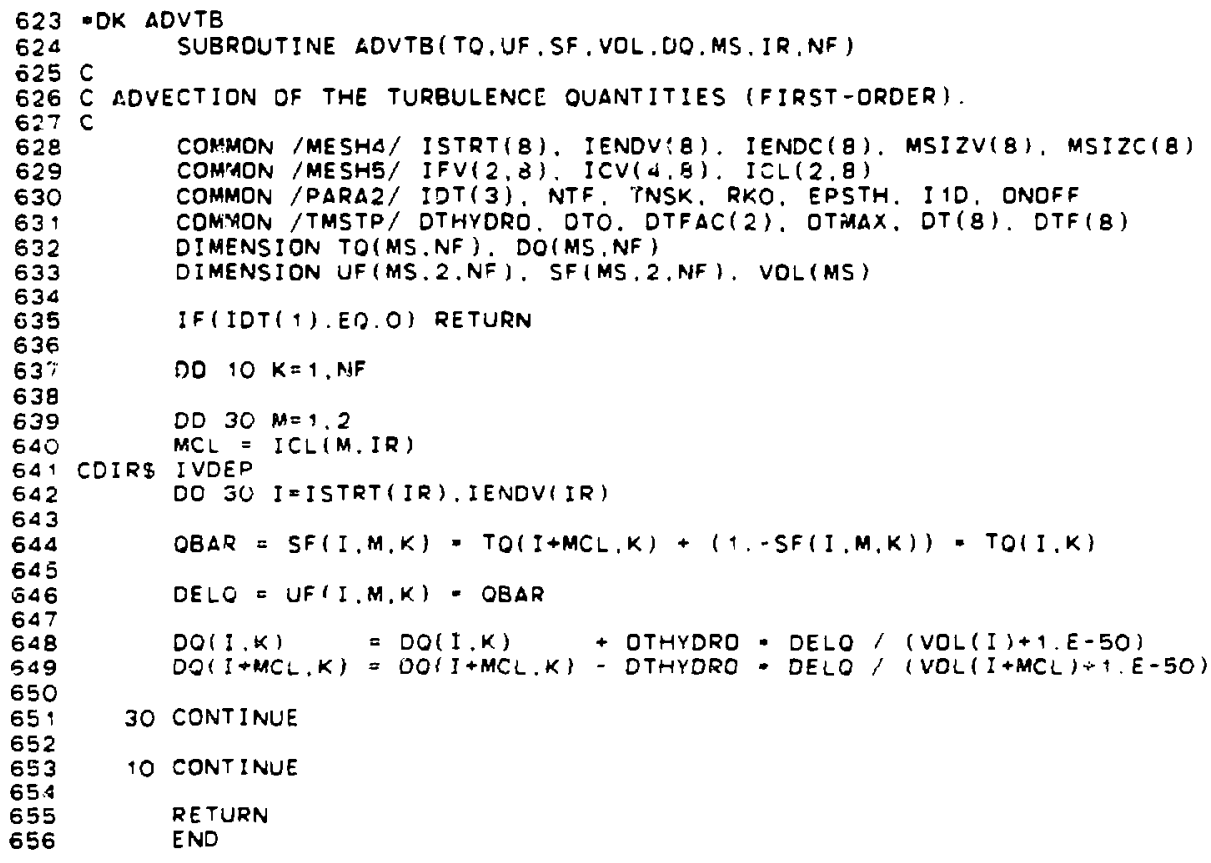




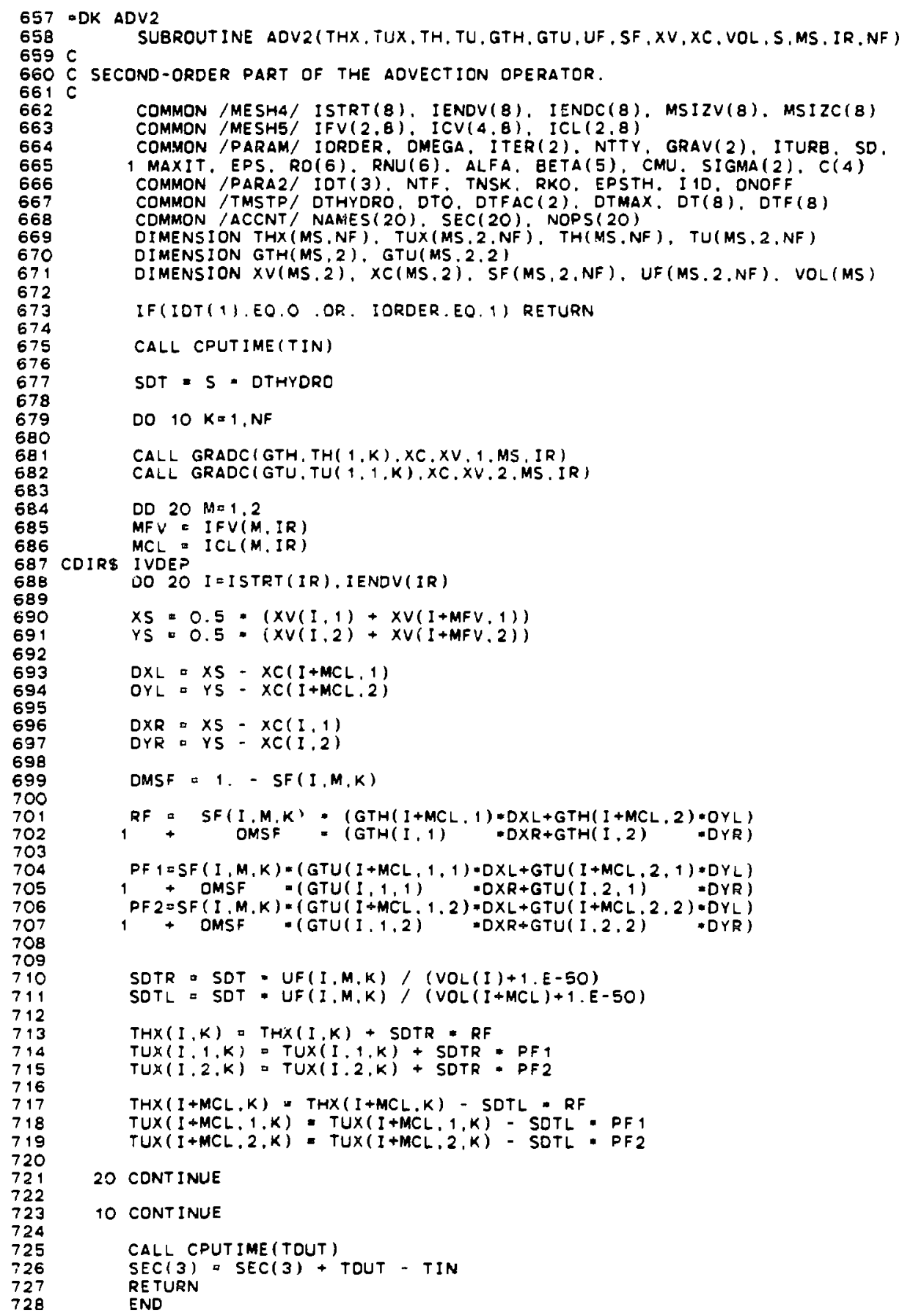




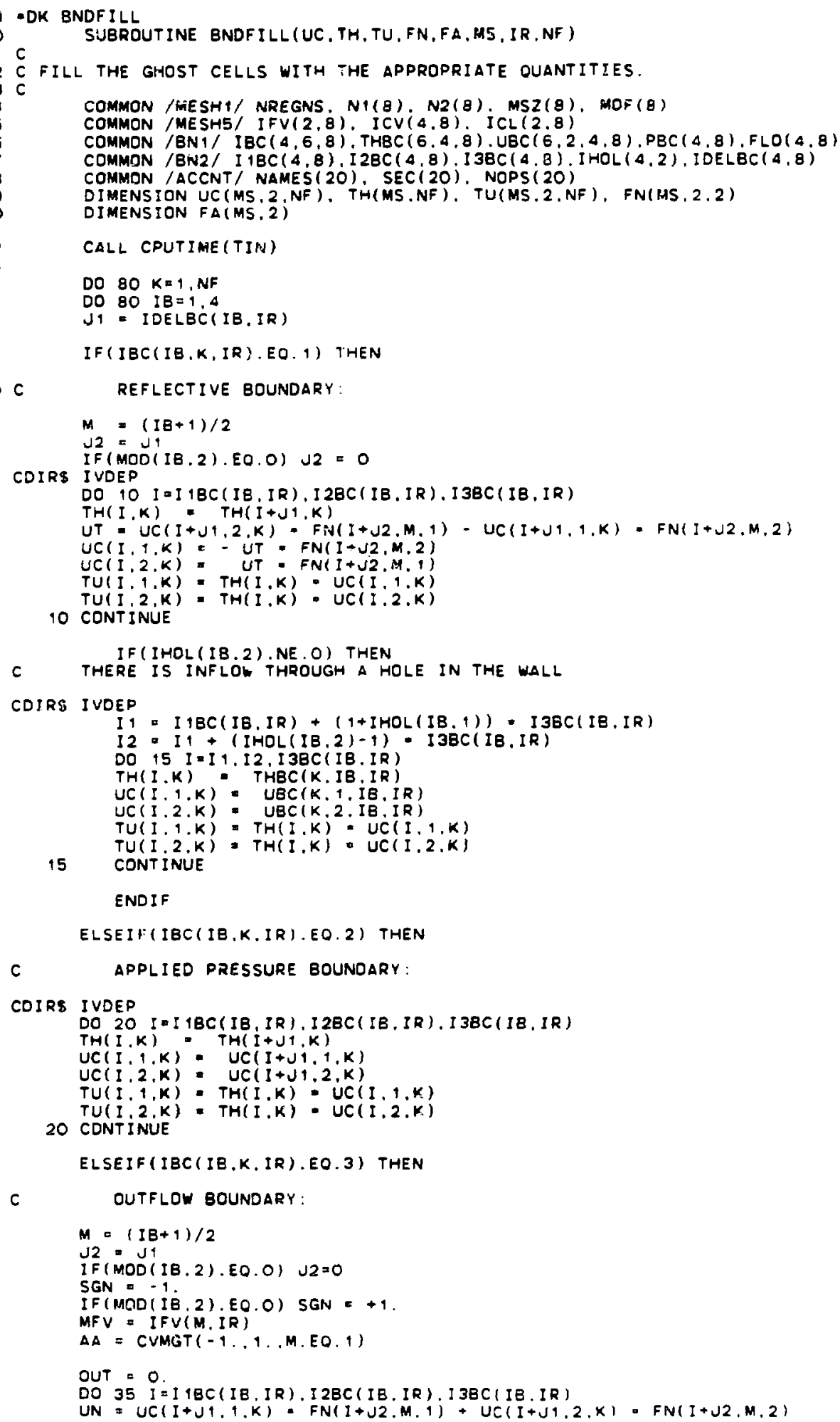




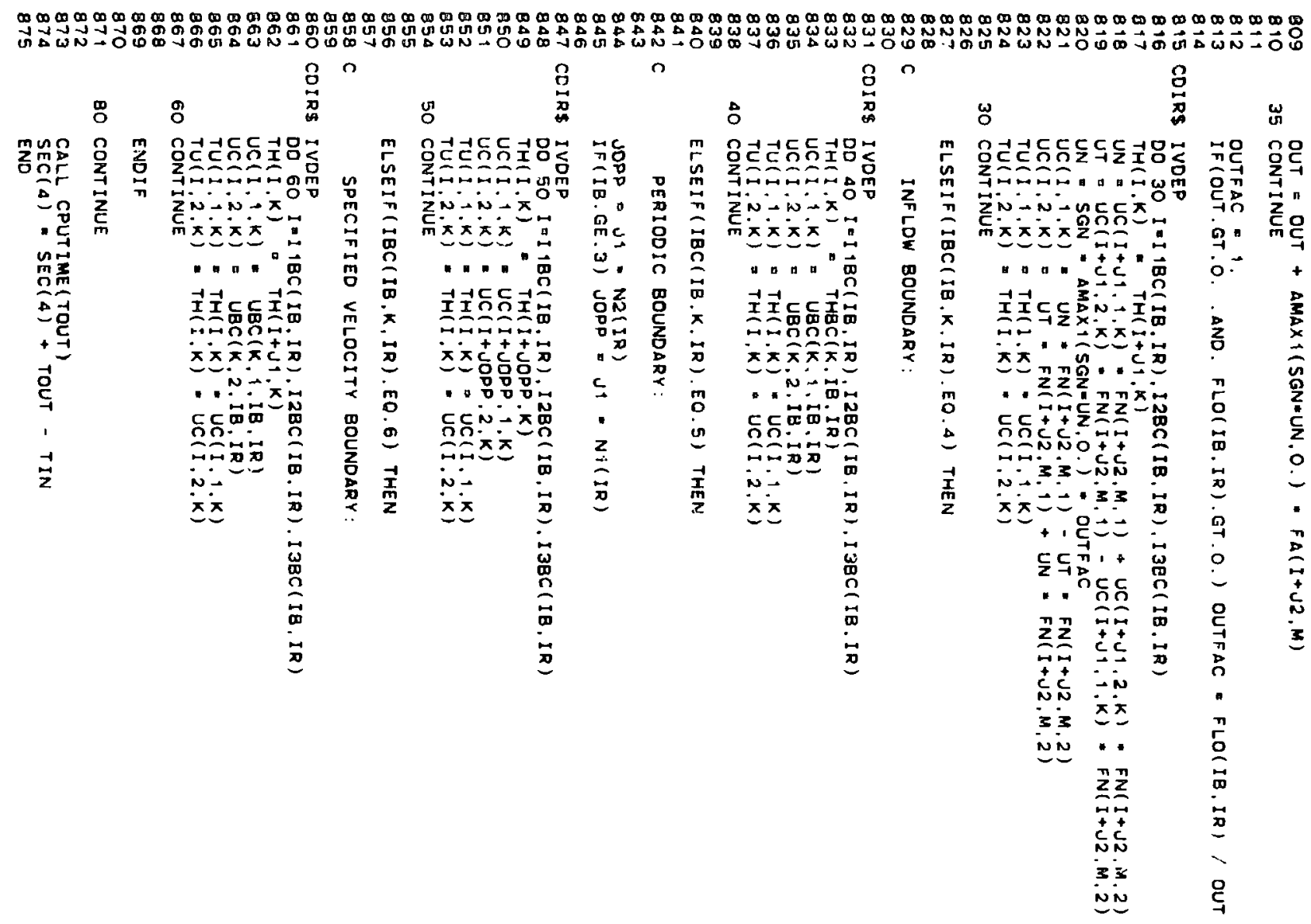




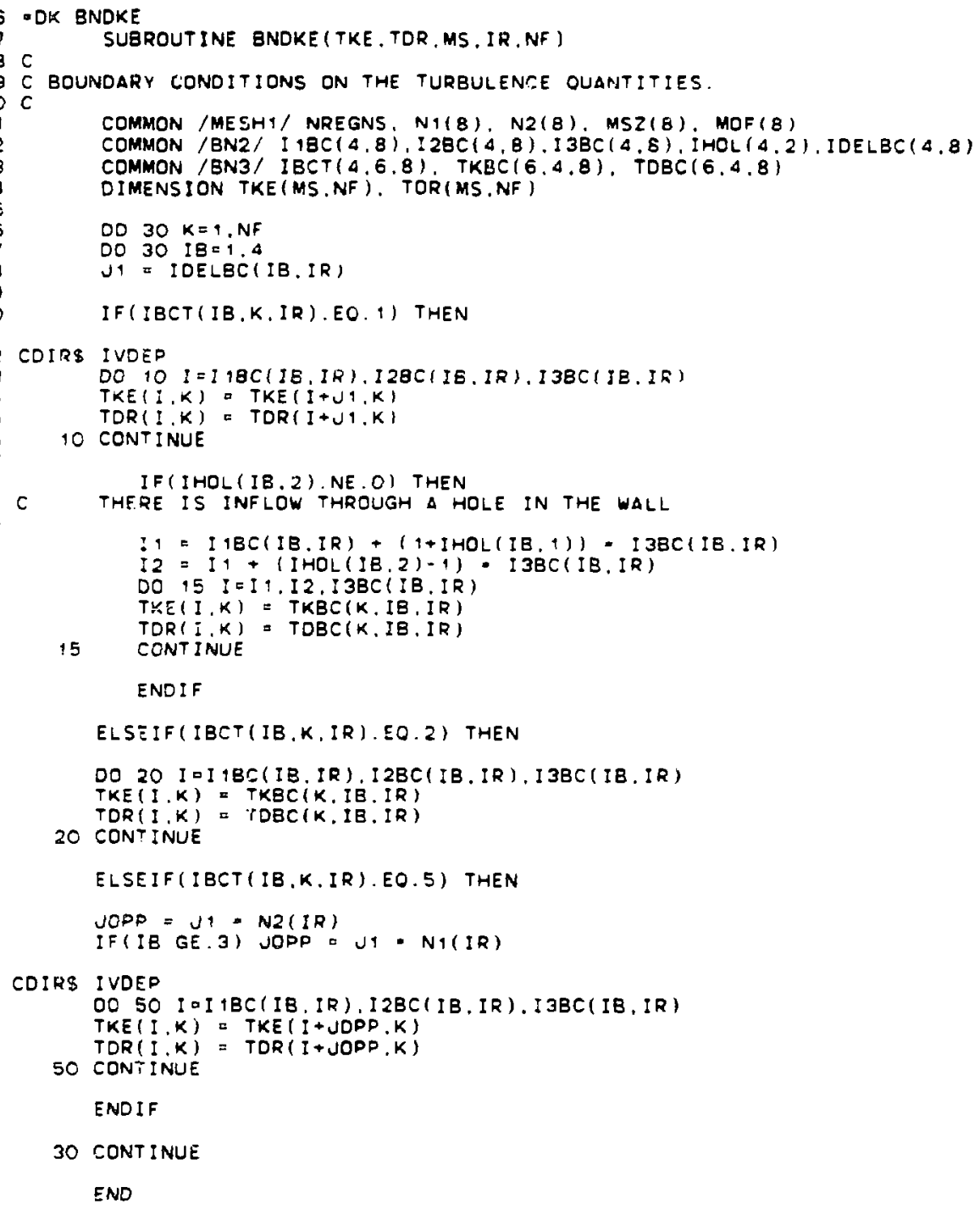



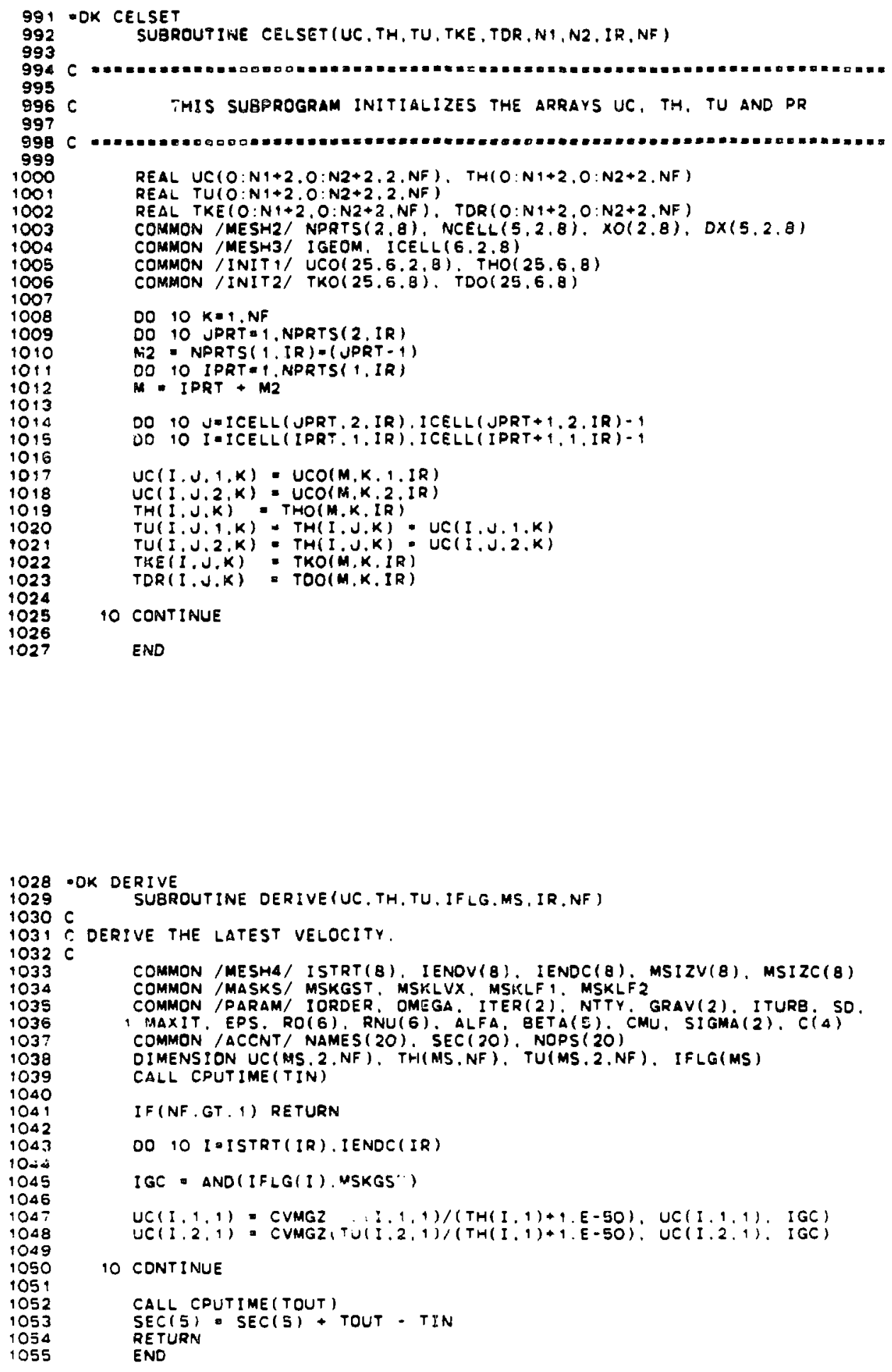


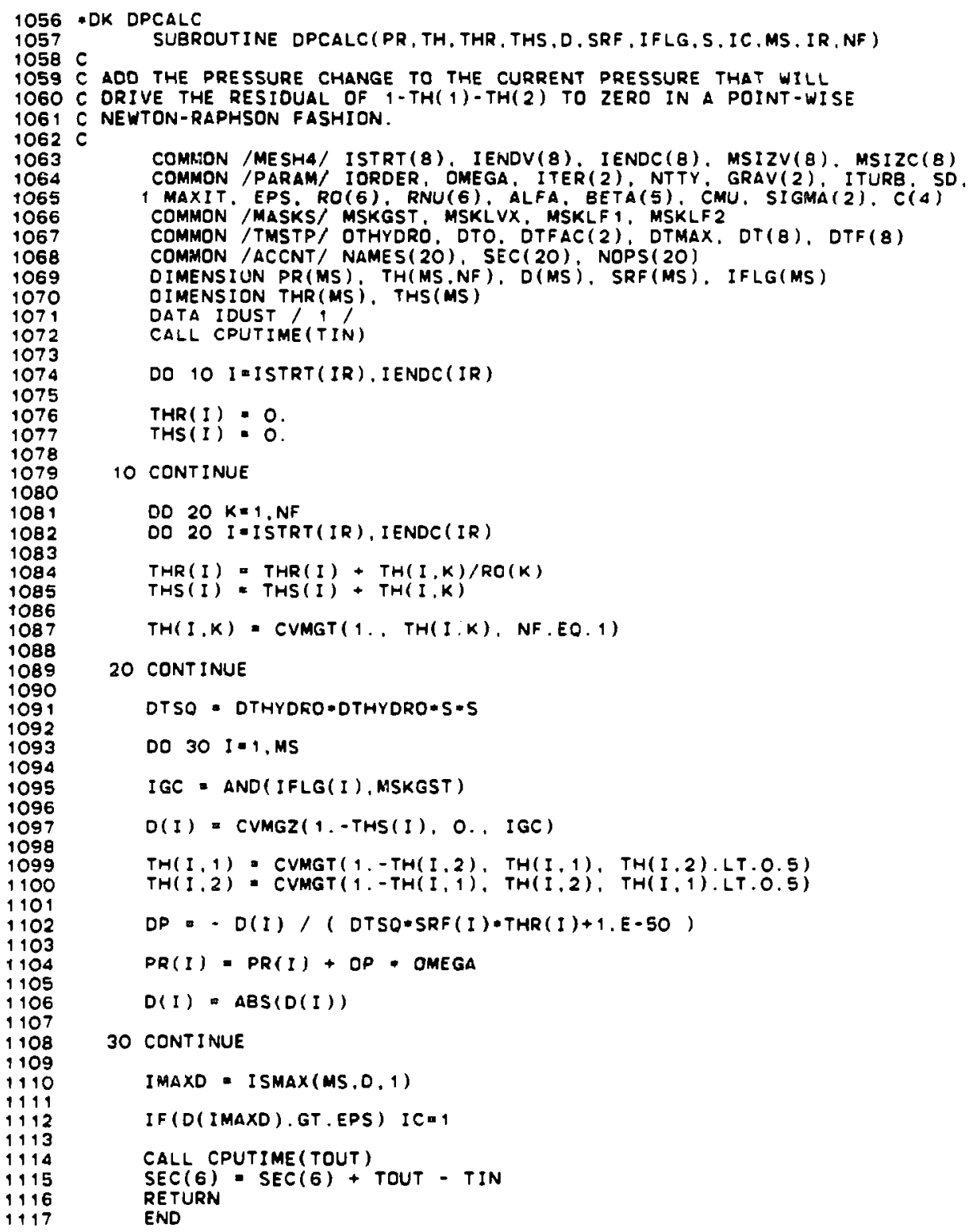




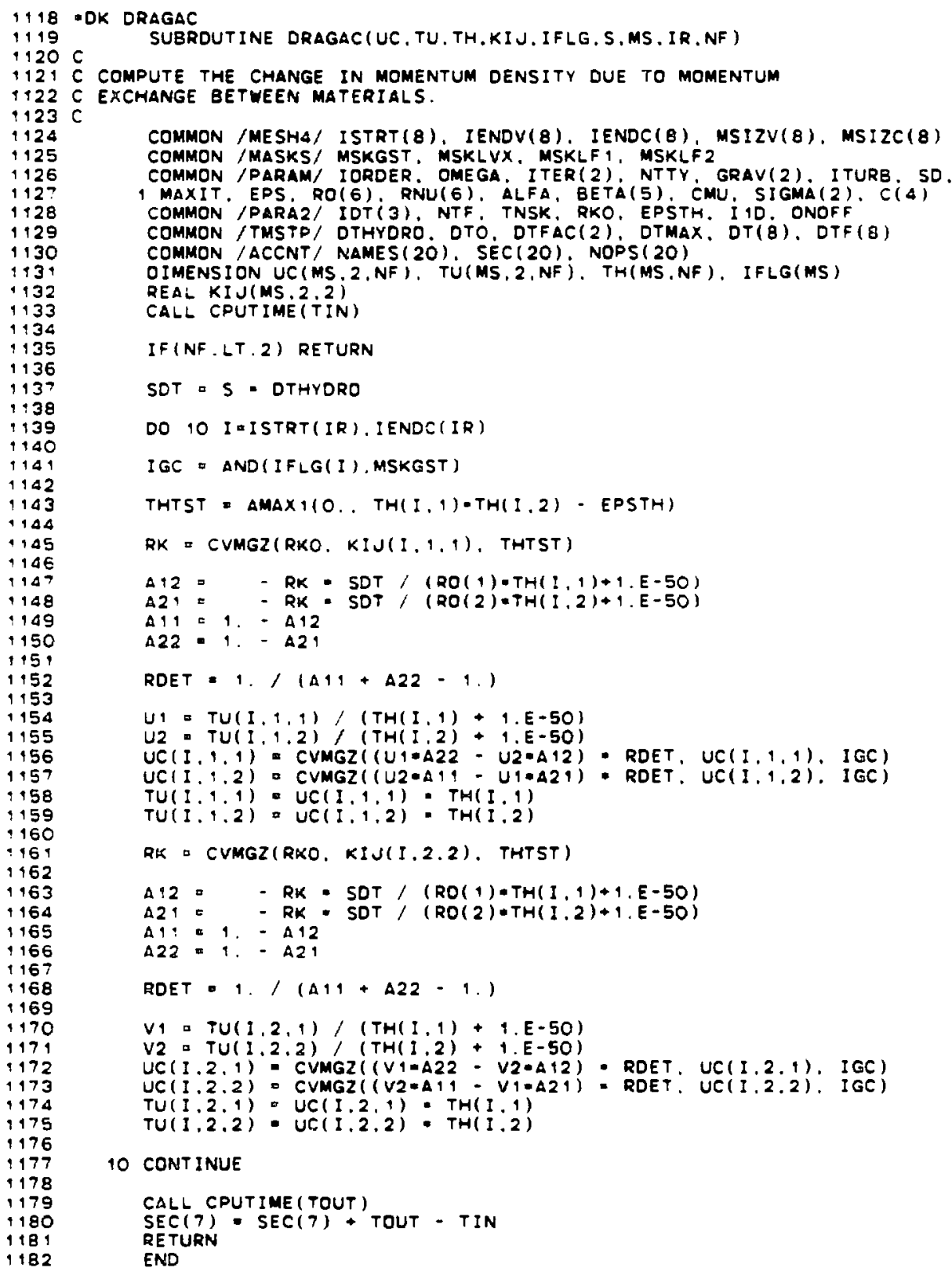




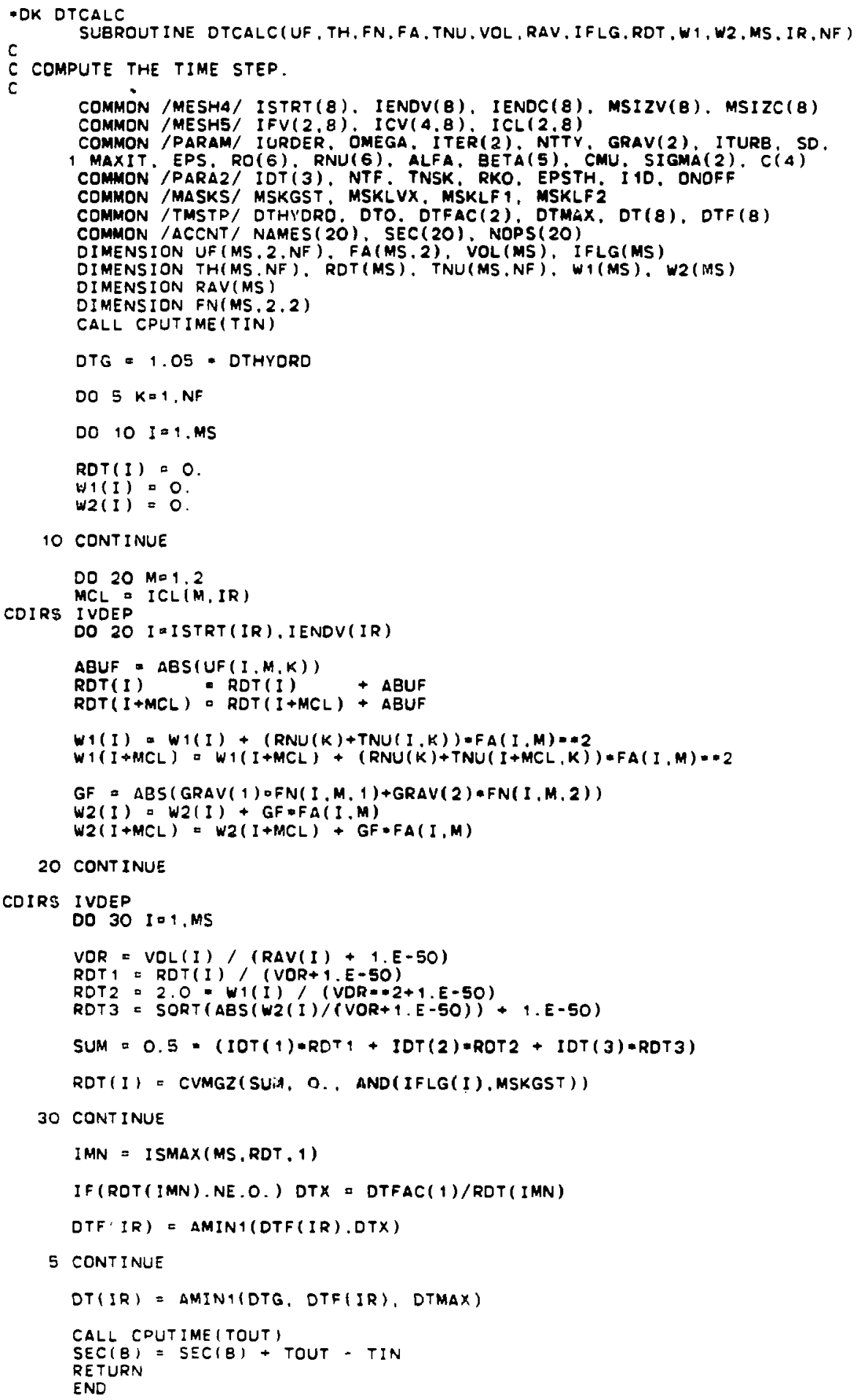




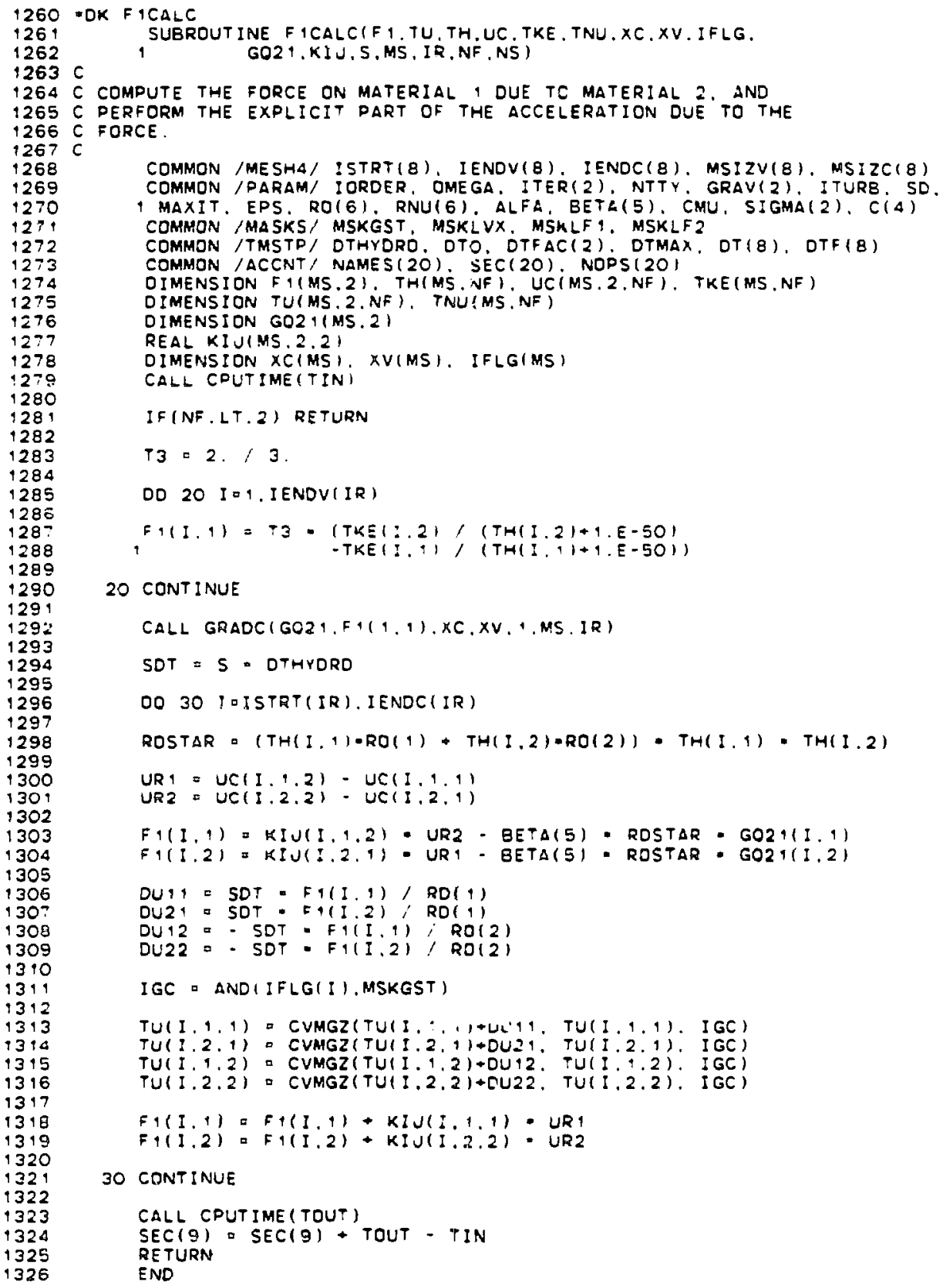




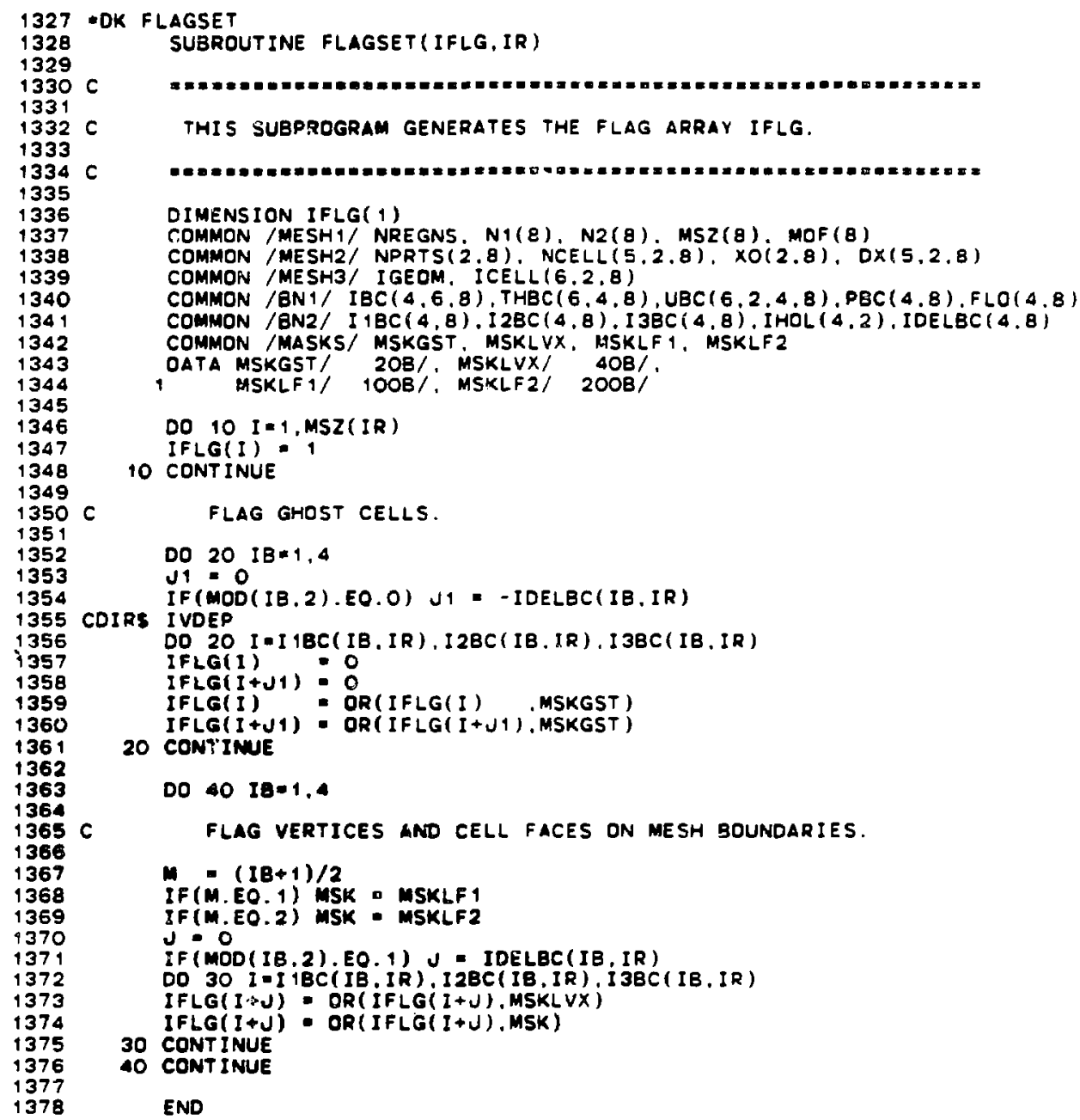




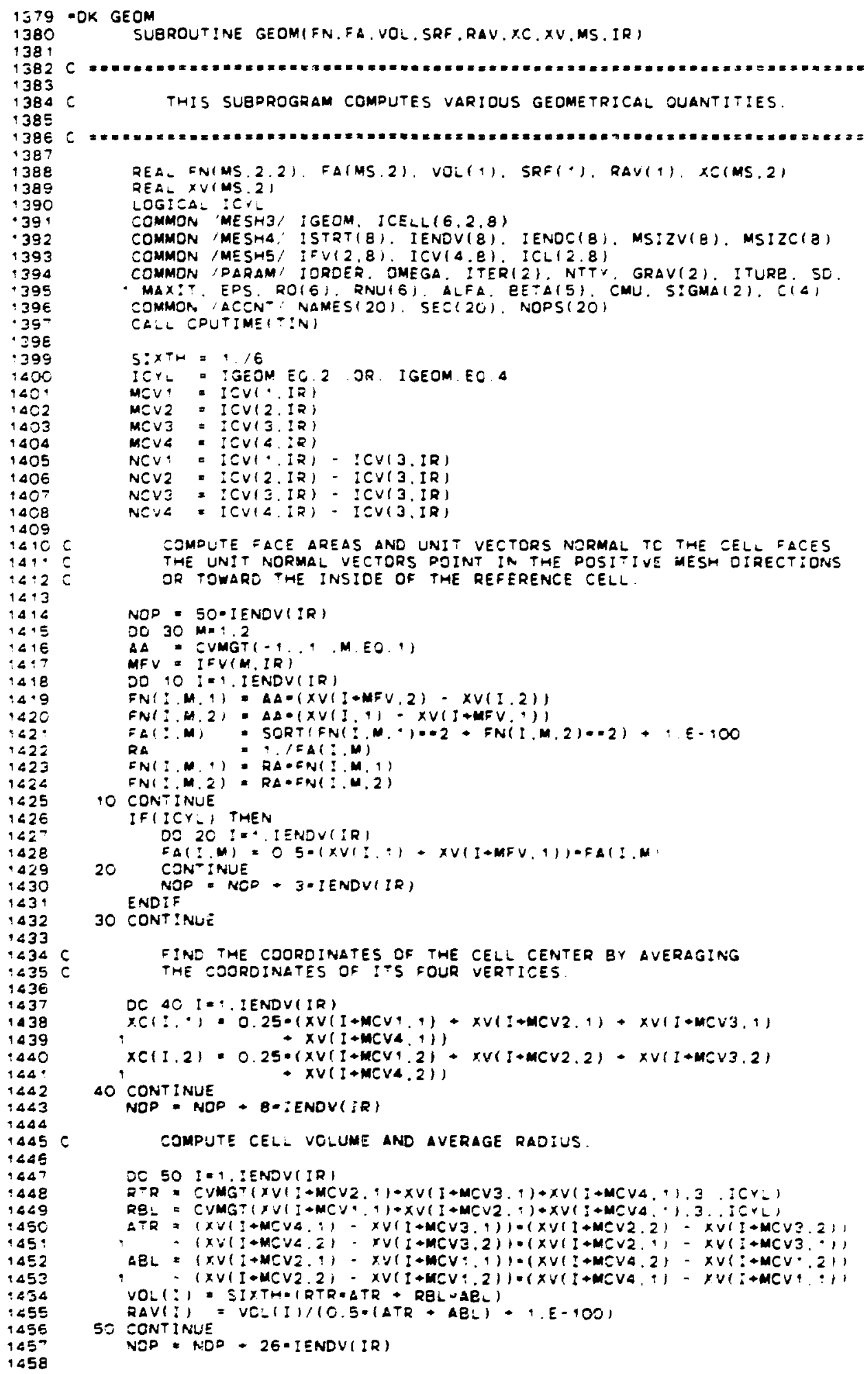




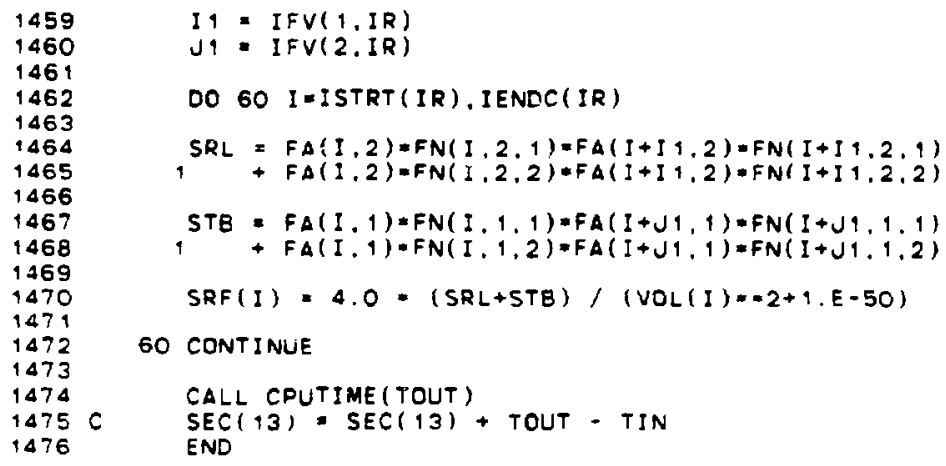




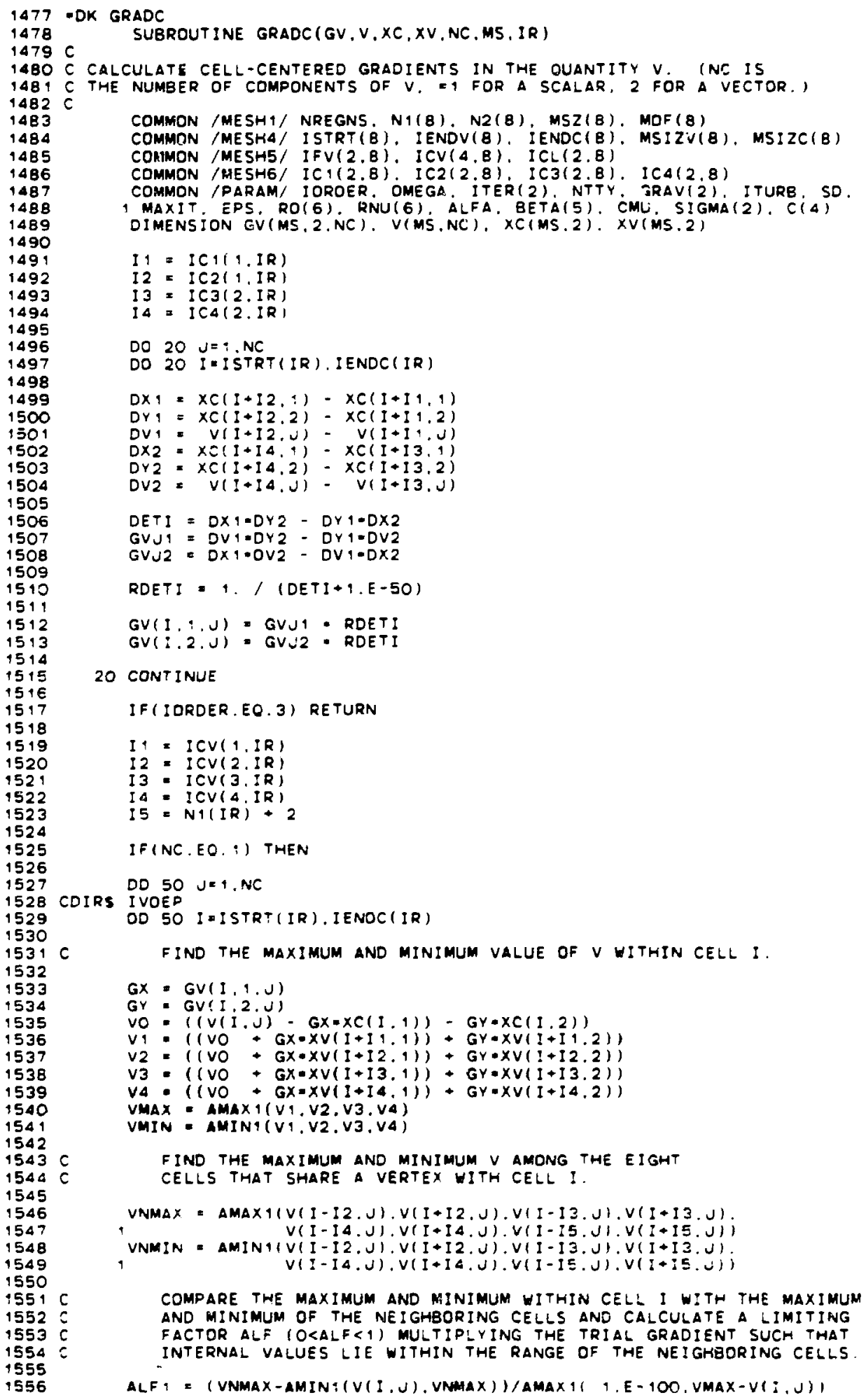




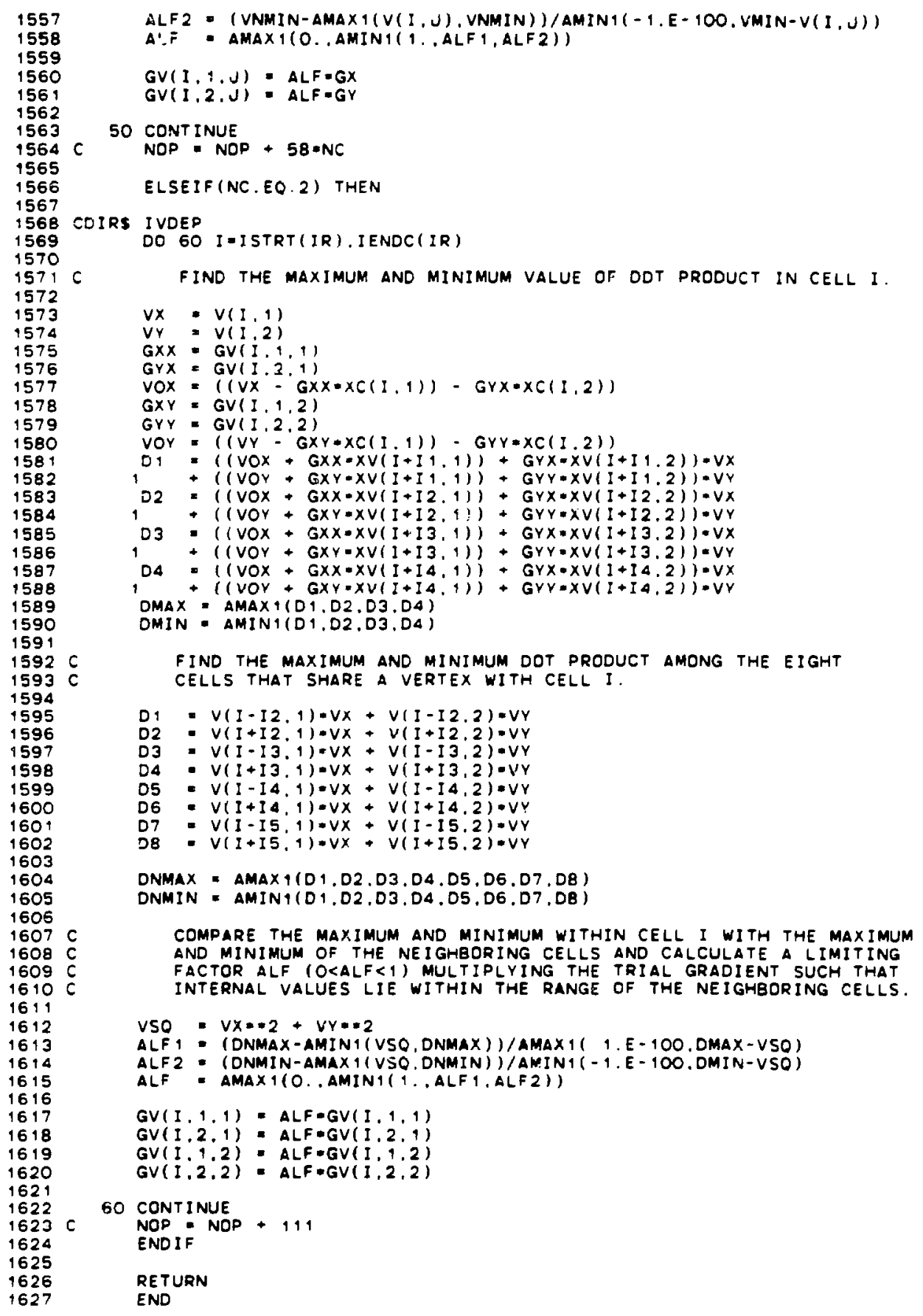




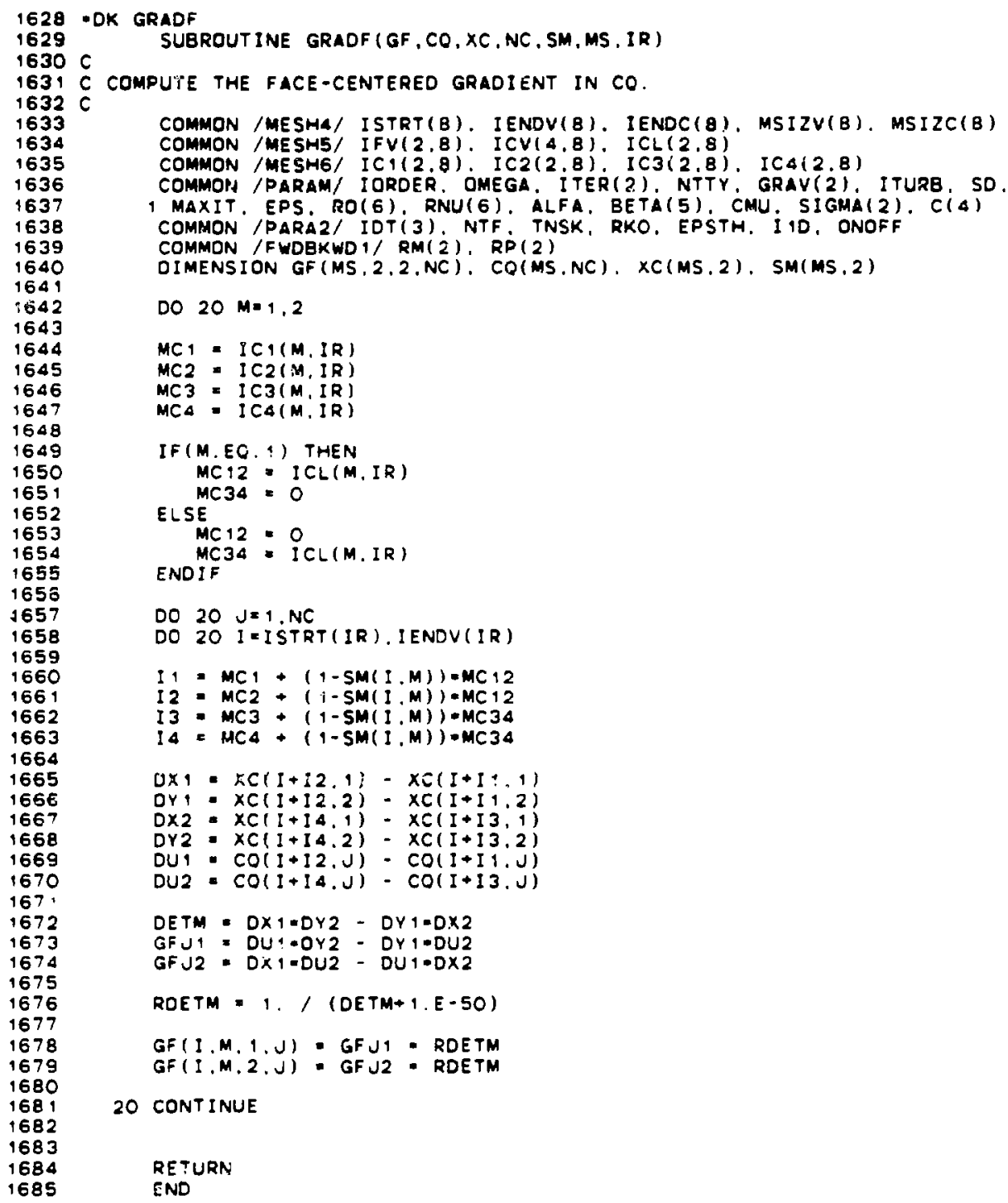




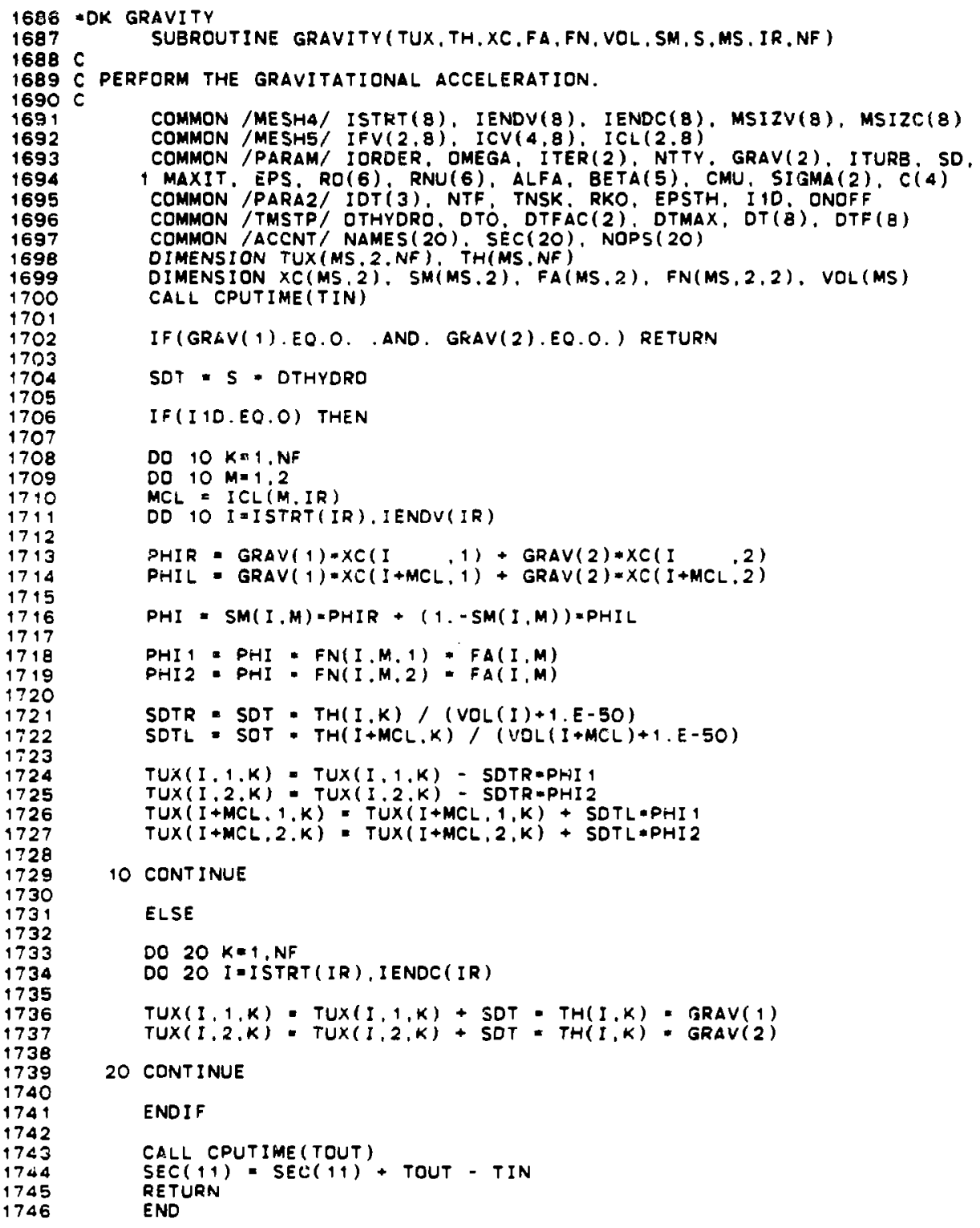




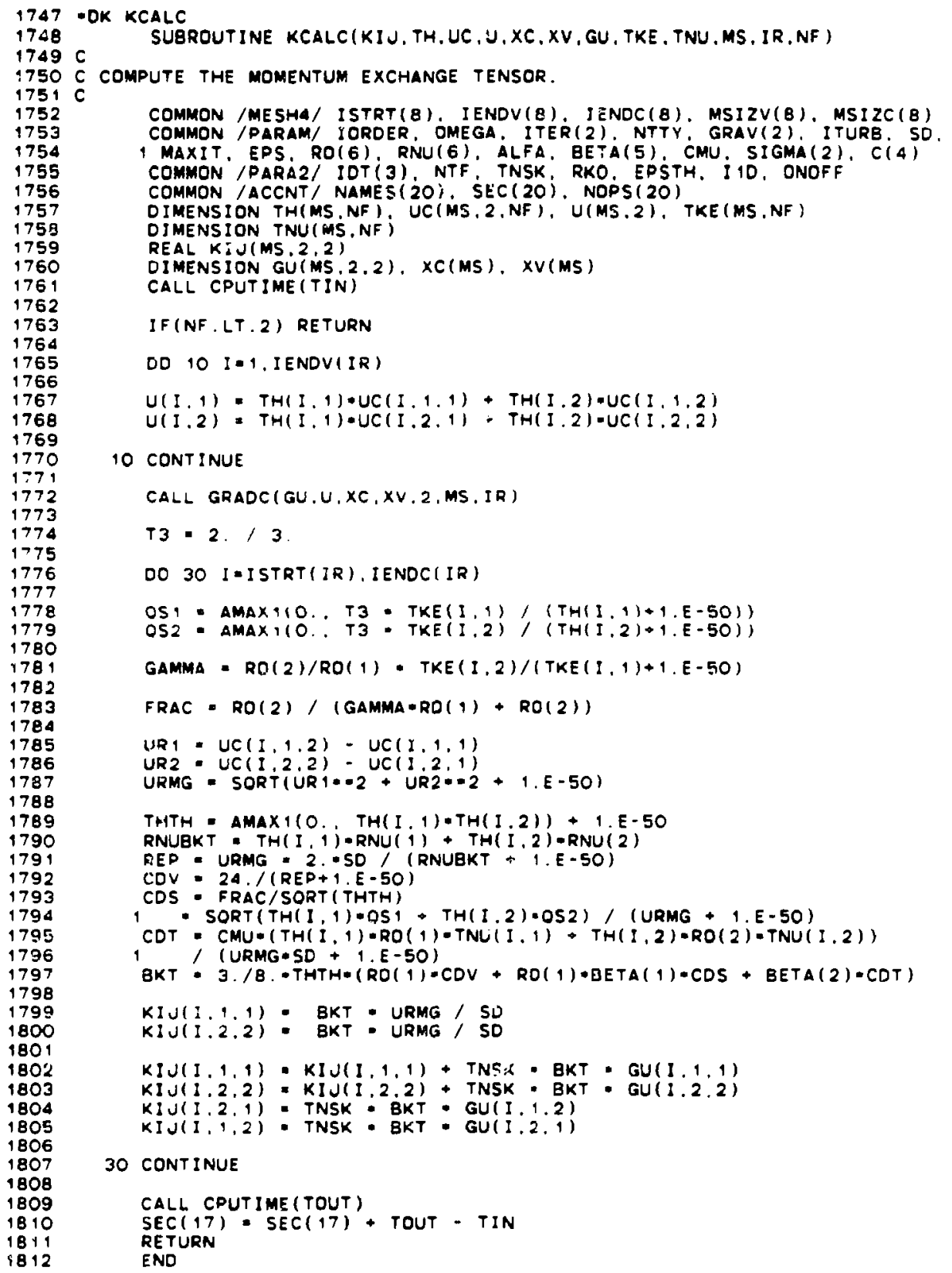




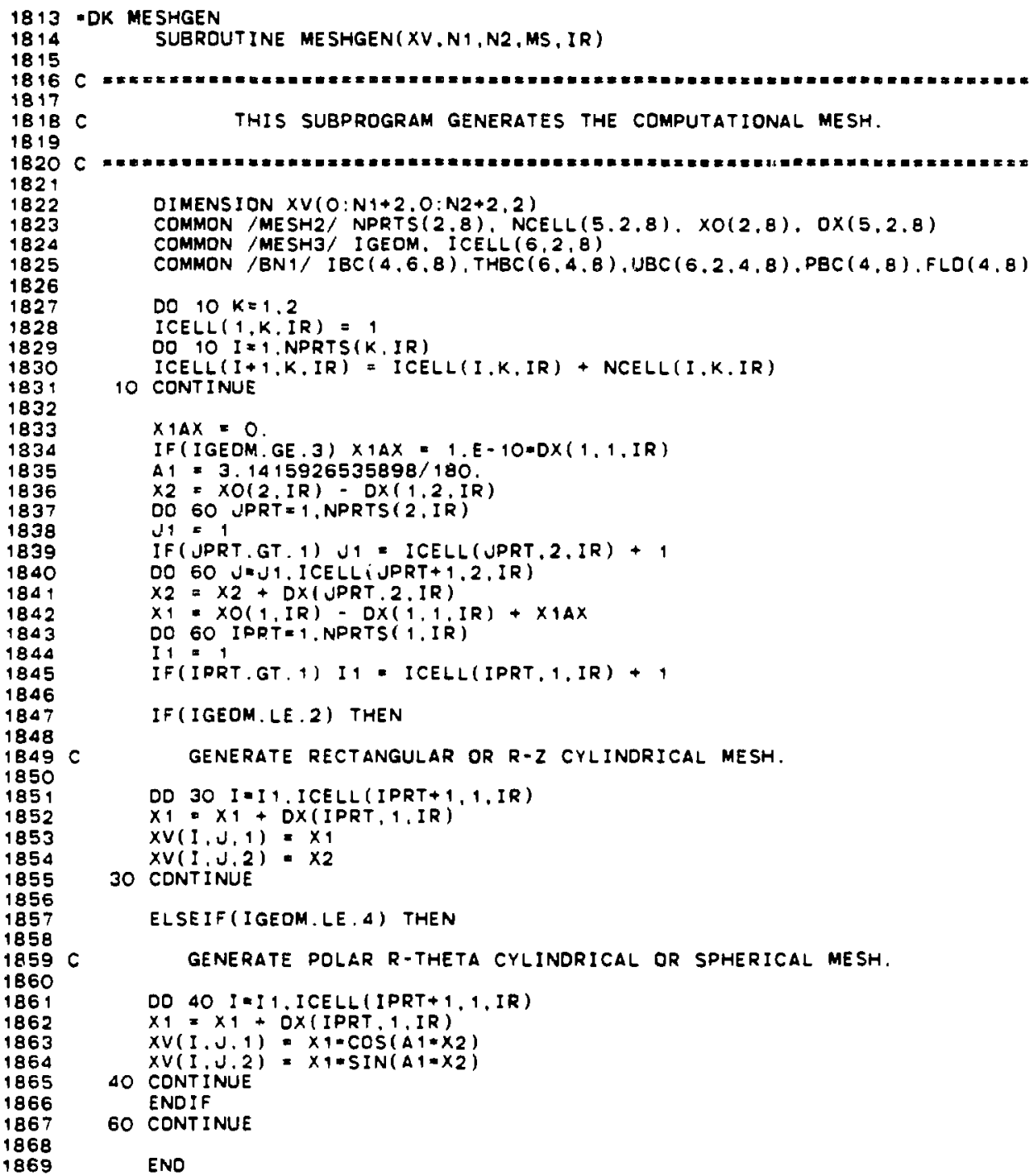




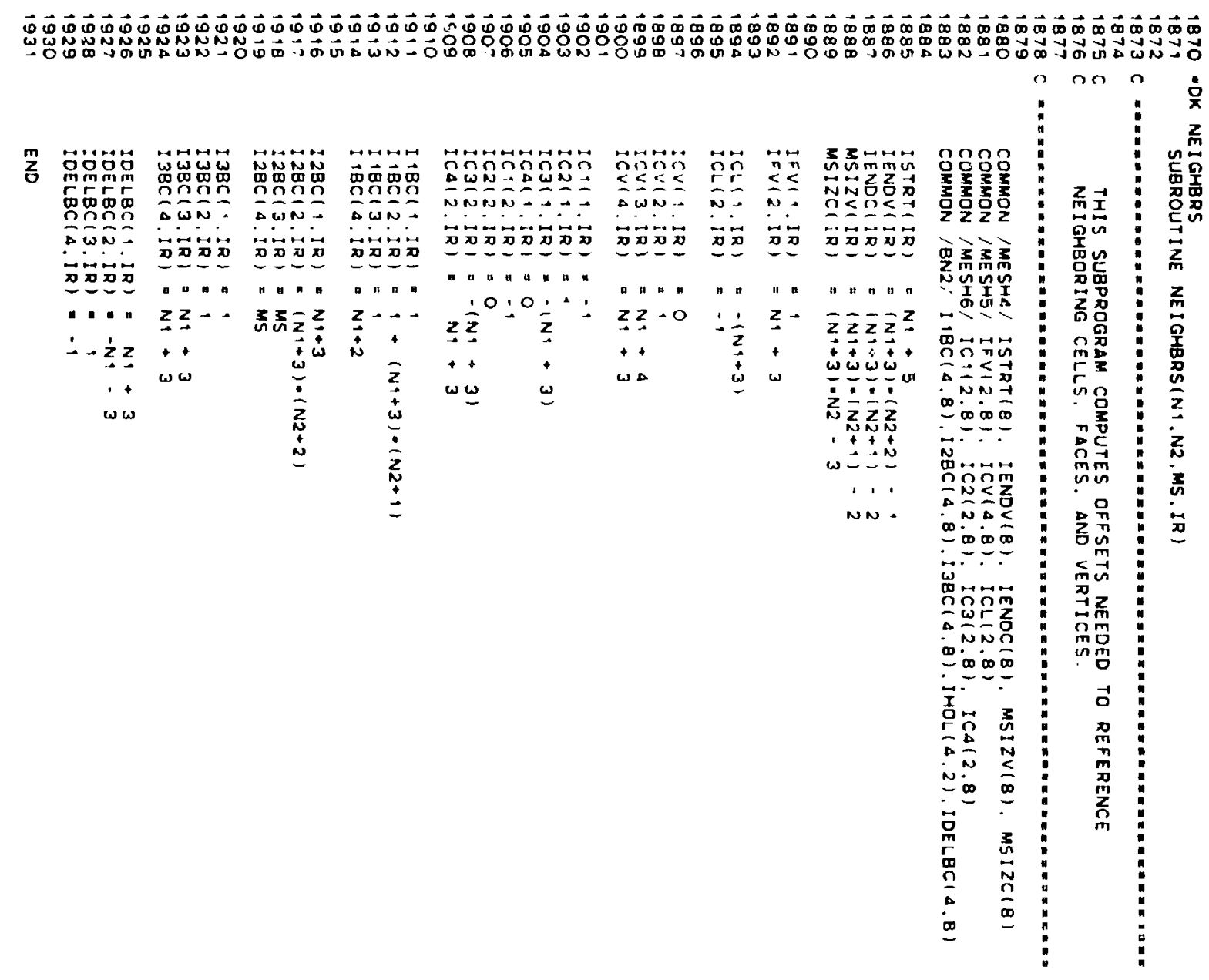




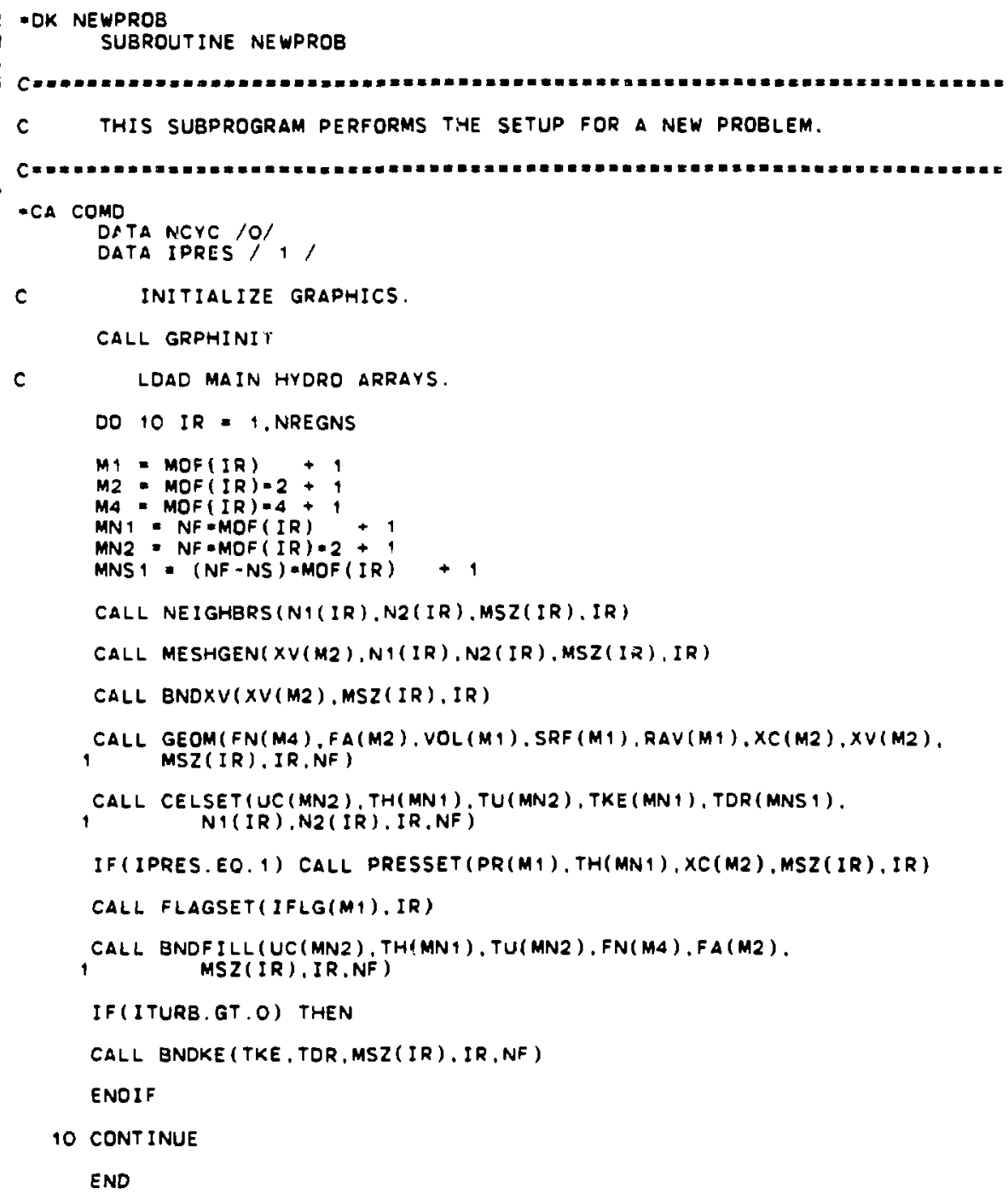




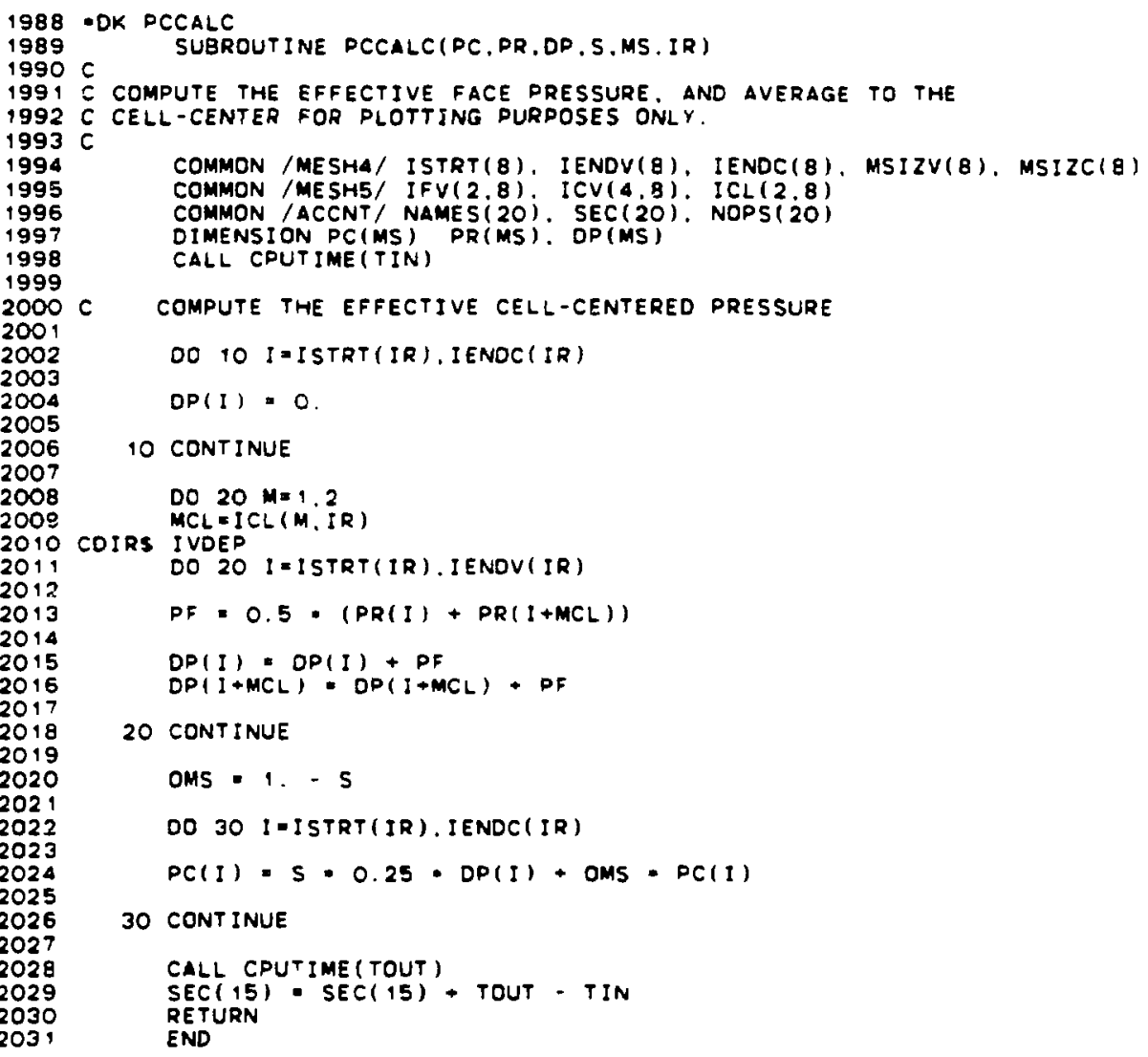




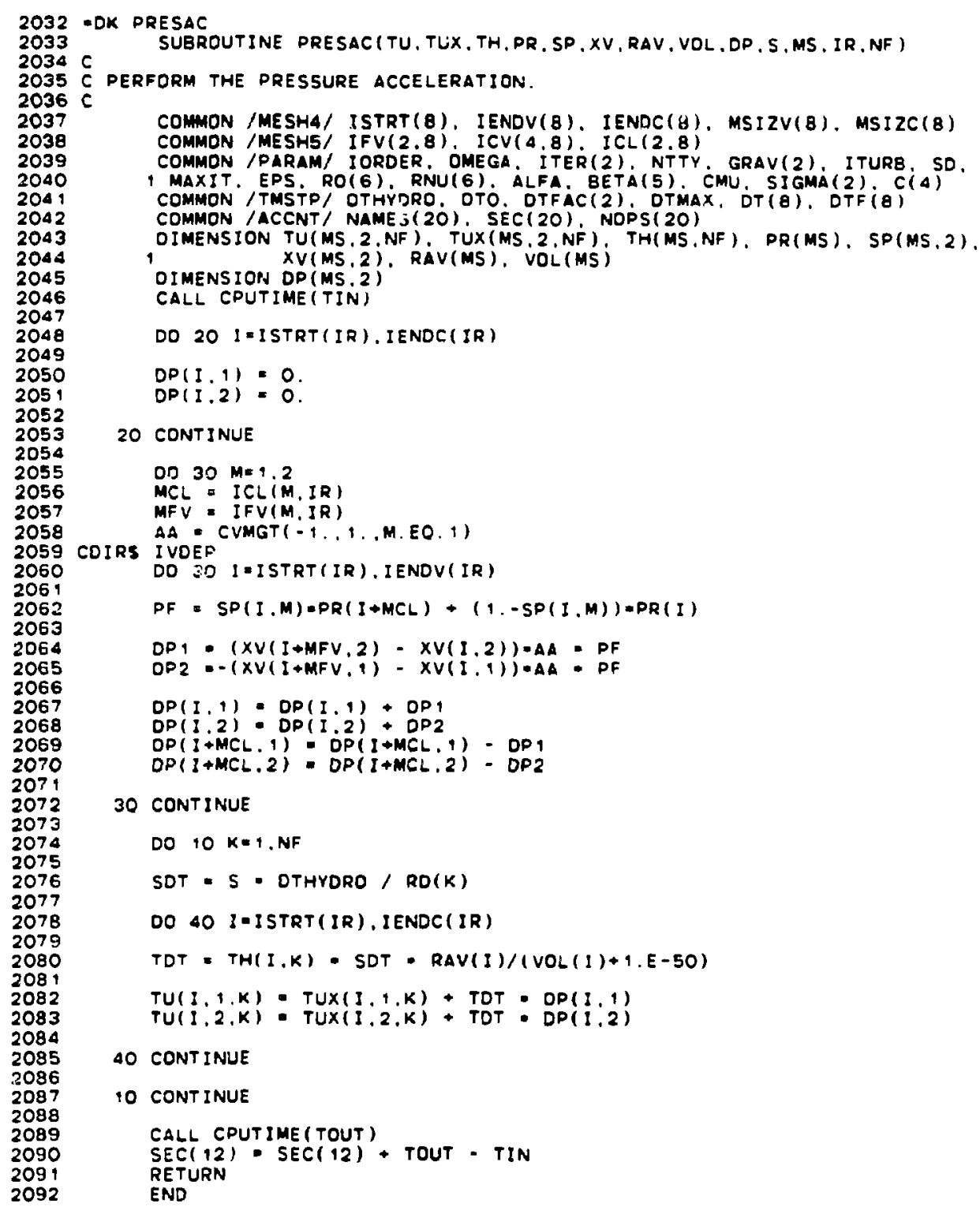



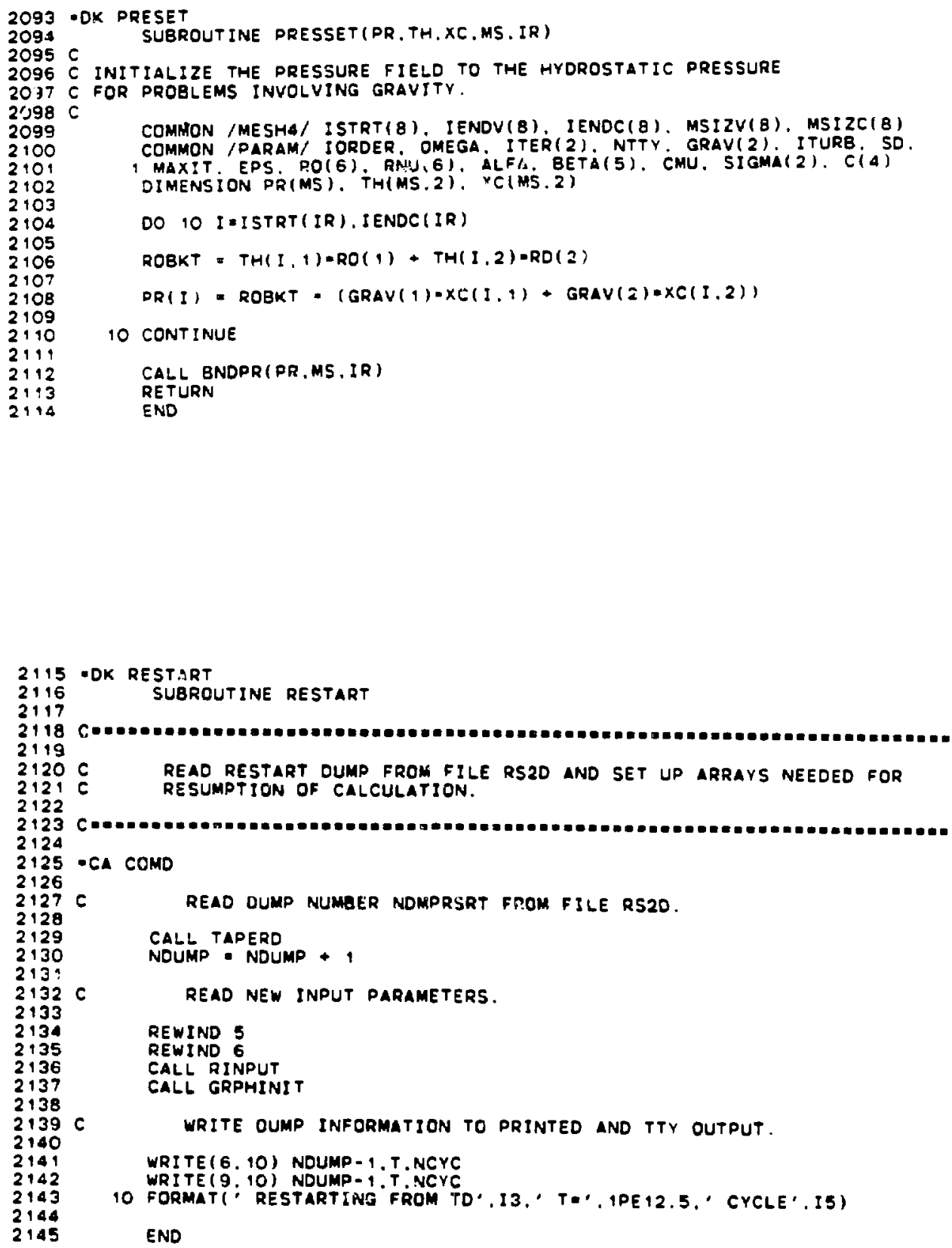


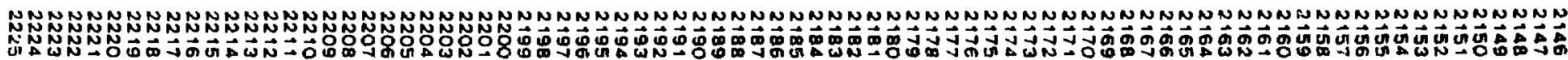

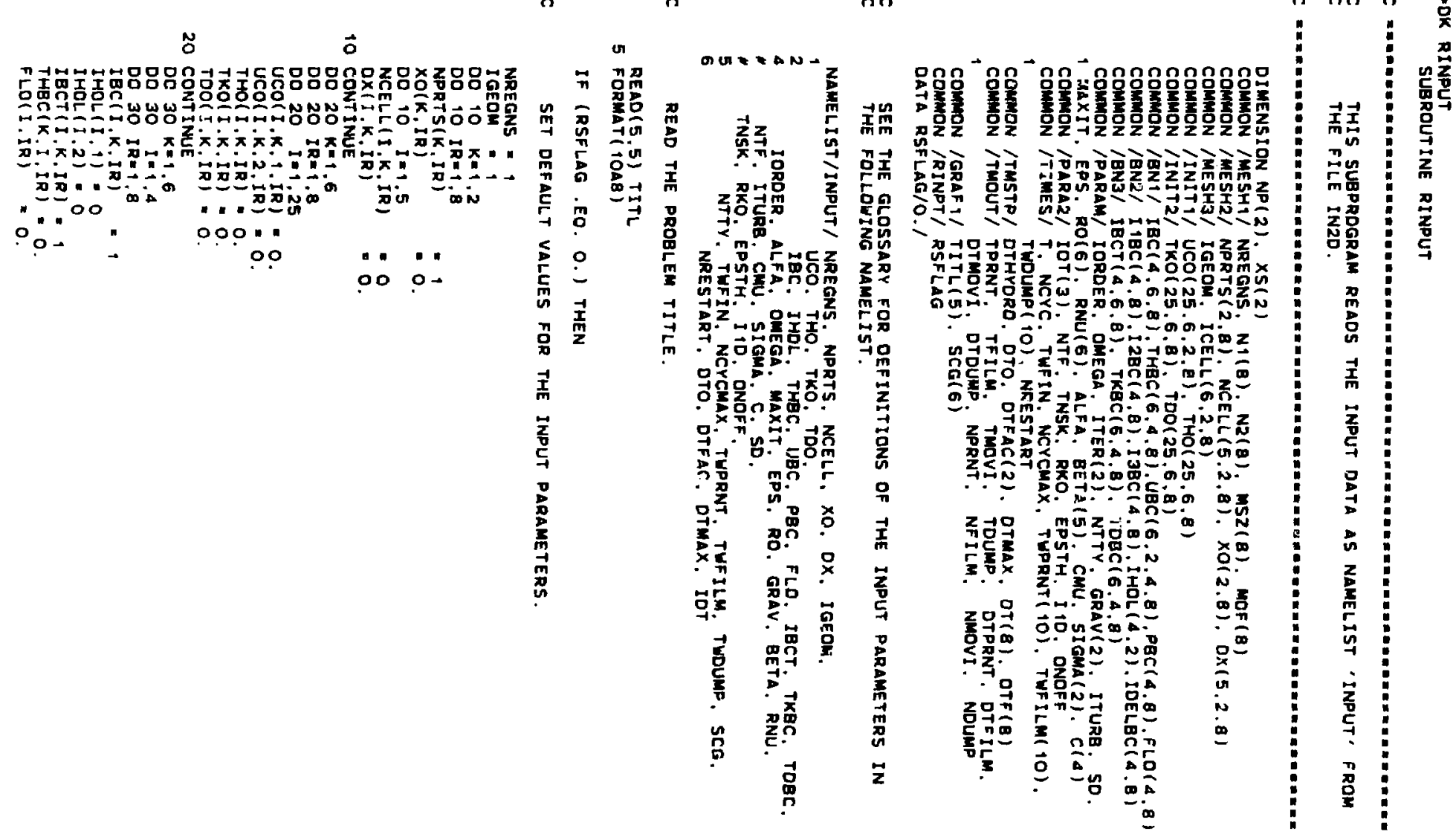


2309

DO 70 IR- 2.8

MOF (IR) $=\operatorname{MOF}(I R-1)+\operatorname{MSZ}(I R-1)$

$7 O$ CONT INUE

END 


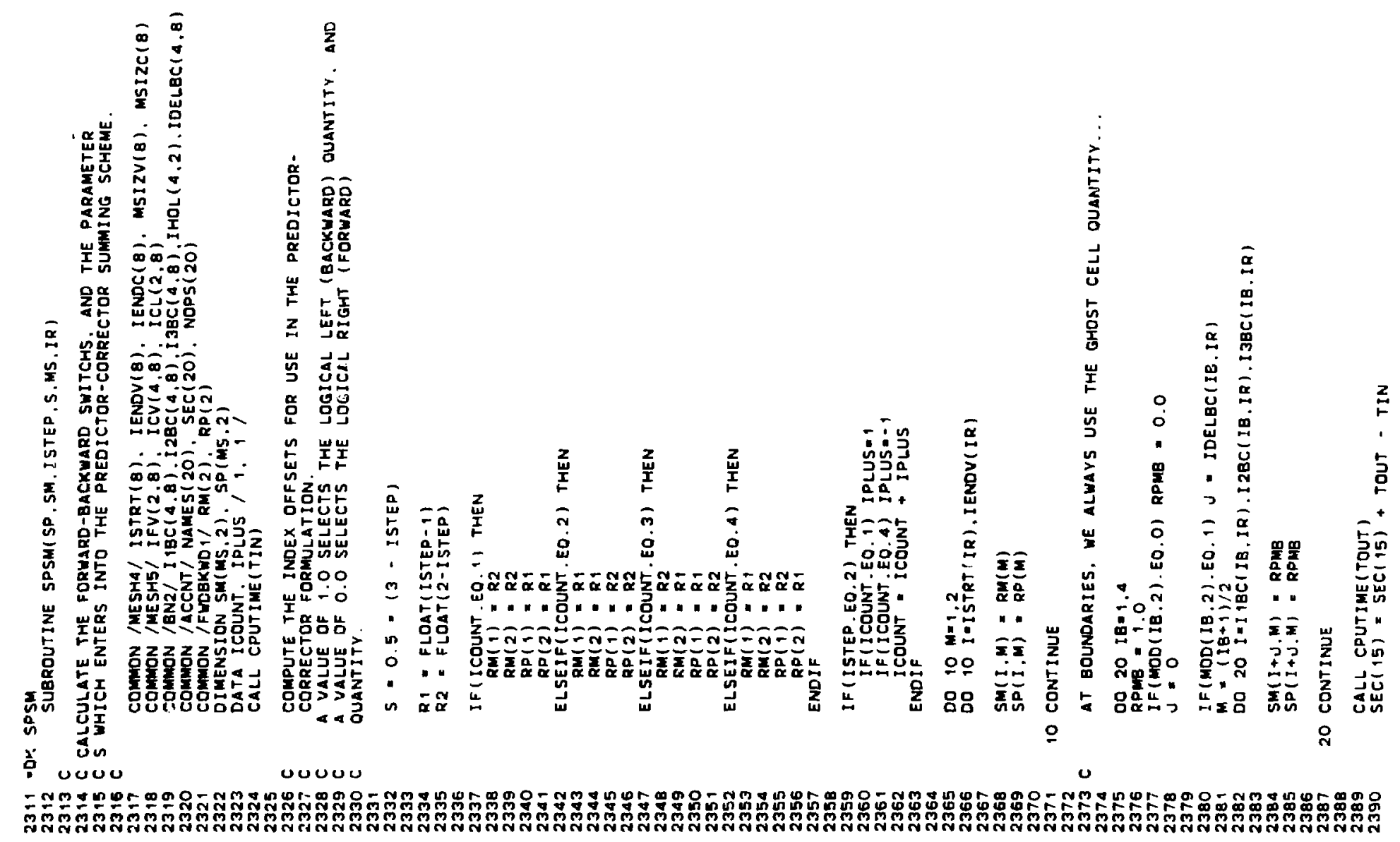




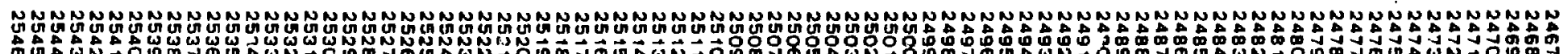

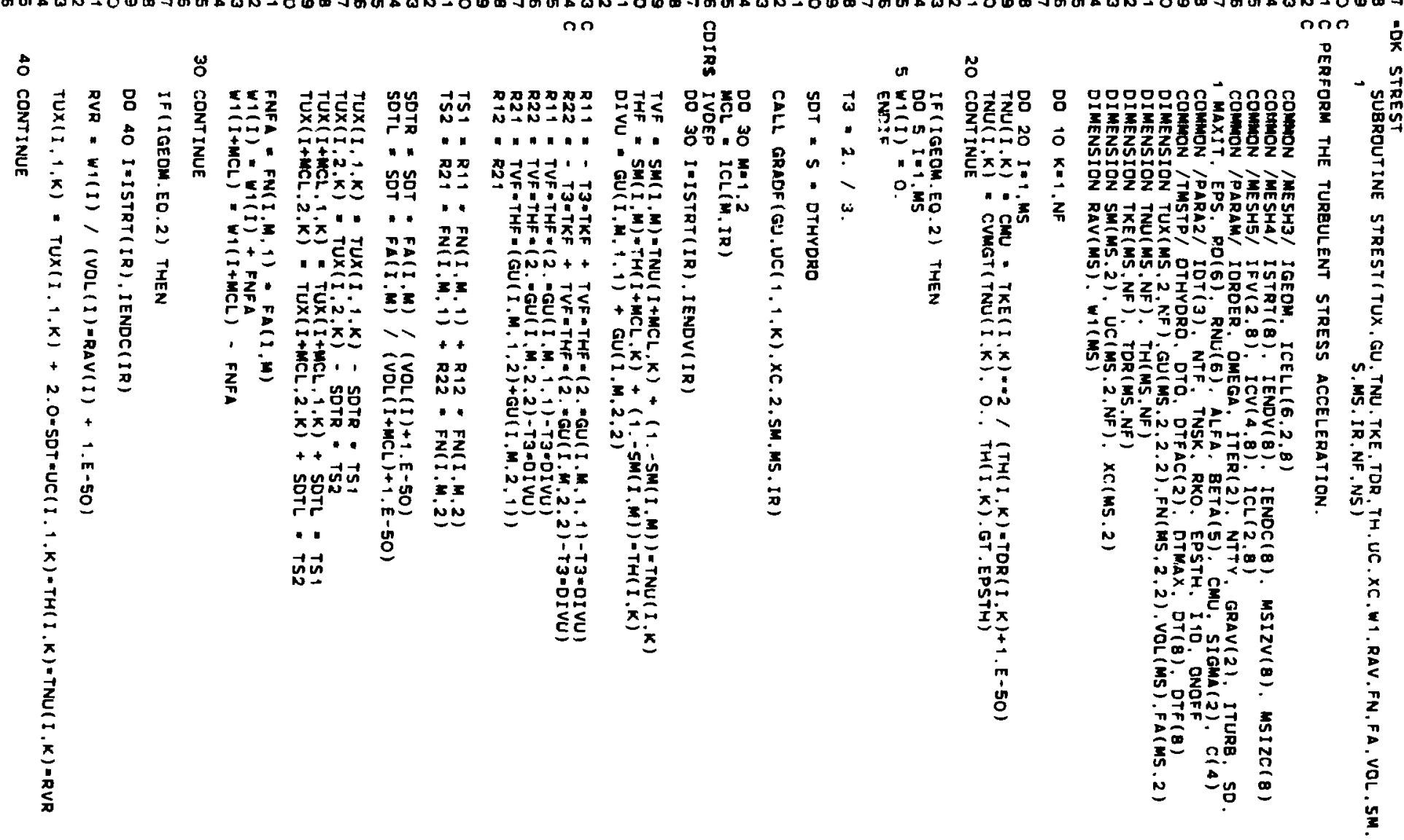




$\begin{array}{lc}2547 & \text { ENDIF } \\ 2548 & \text { CONTINUE } \\ 2549 & 10 \text { CONT } \\ 2550 & \text { RETURN } \\ 2551 & \text { END } \\ 2552 & \text { EN }\end{array}$

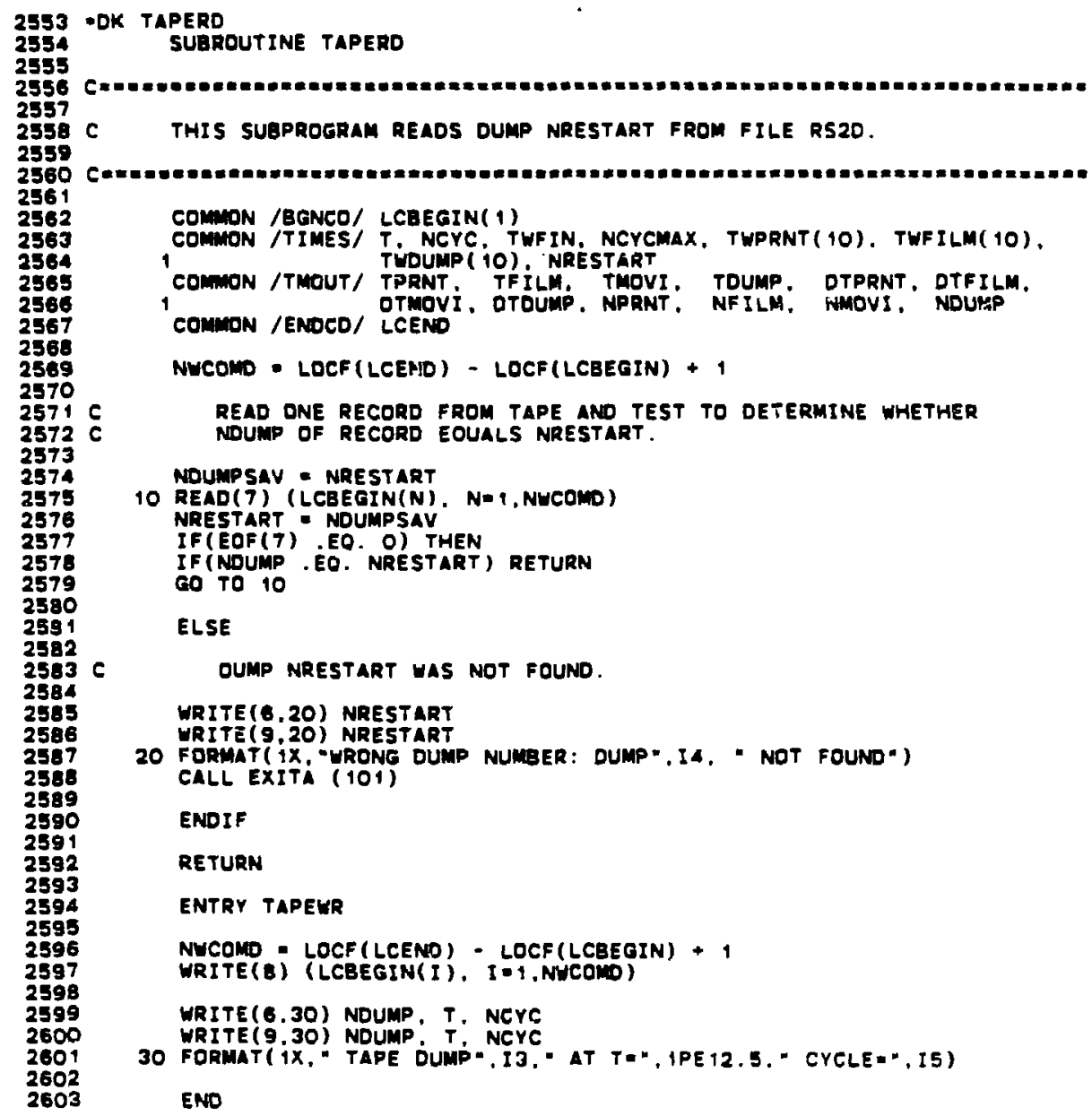




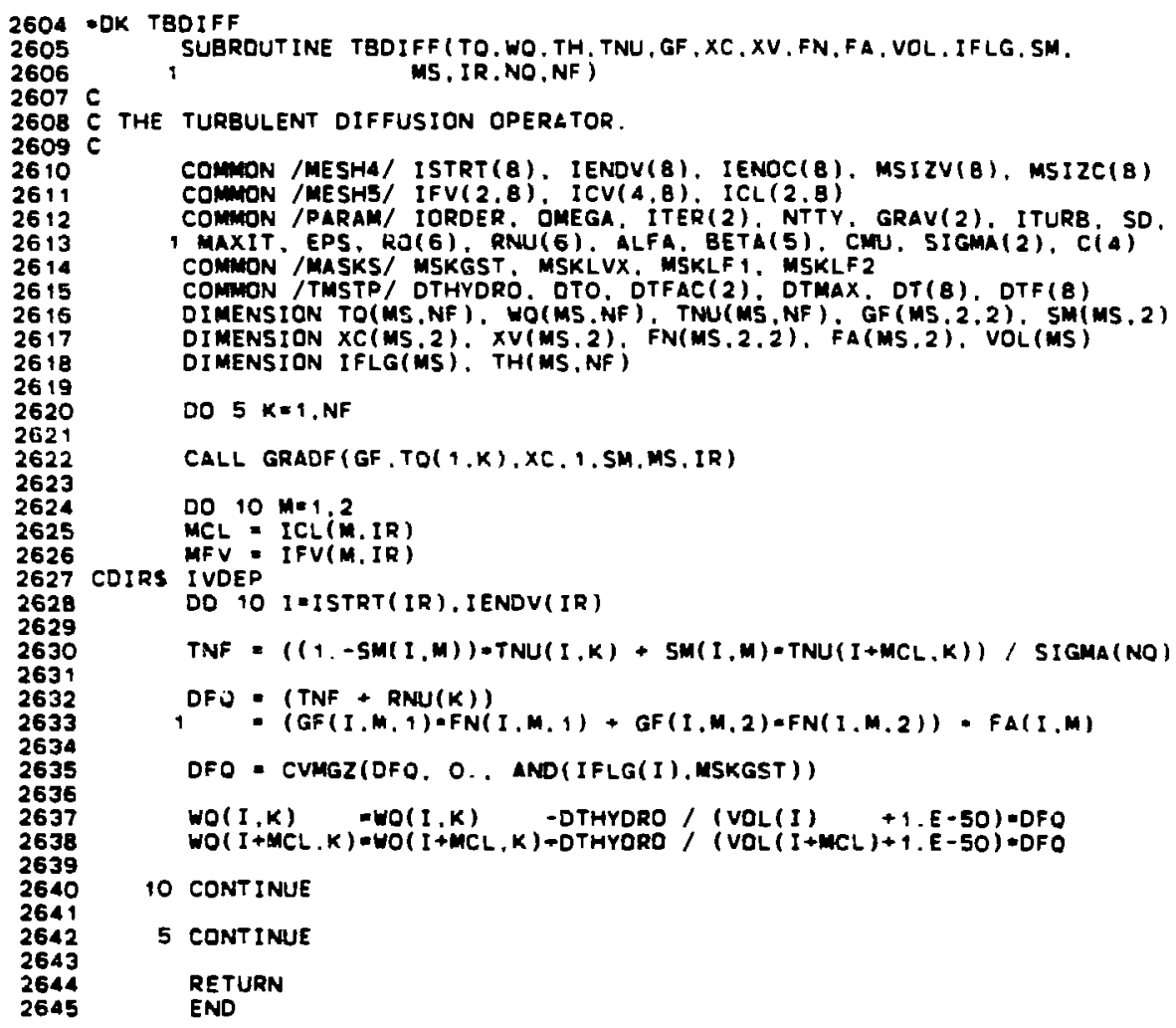




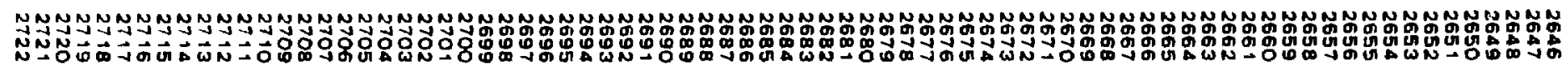
กอ

on

$\overrightarrow{0}$ o

ธิ

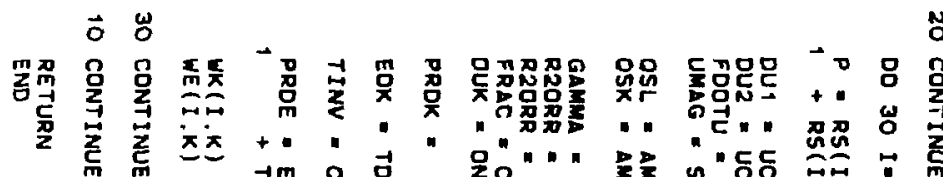

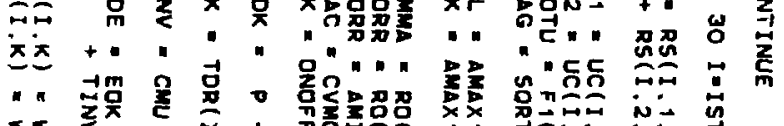

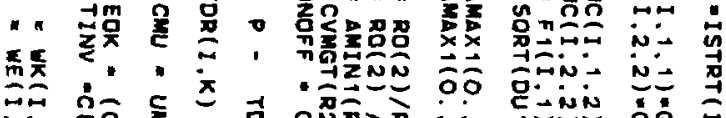

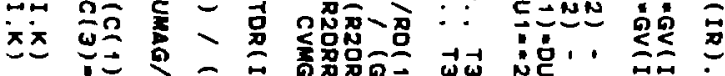
++ dy

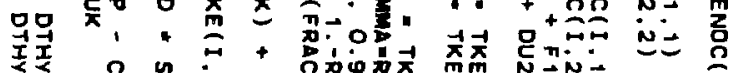

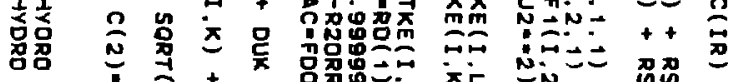

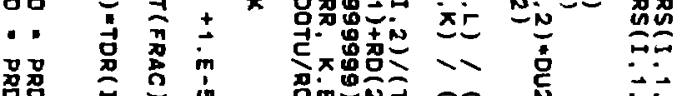

总总

요

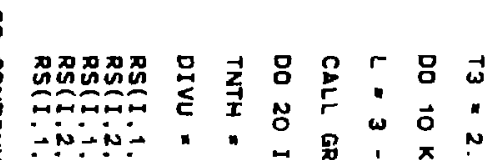

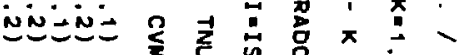
"I,", 要

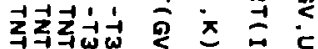

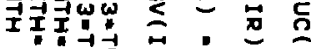

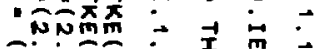

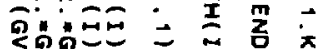

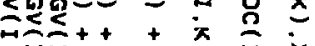

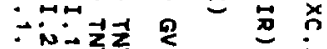
iNo-

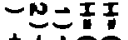
a

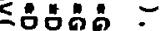

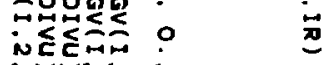




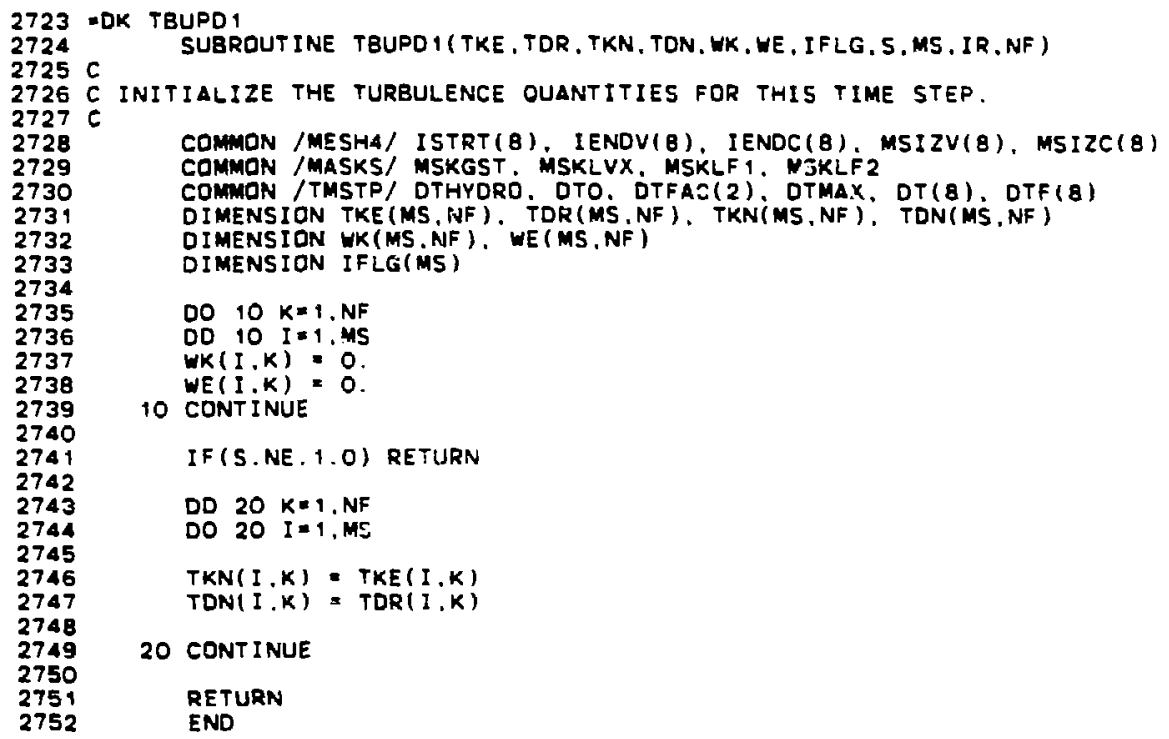









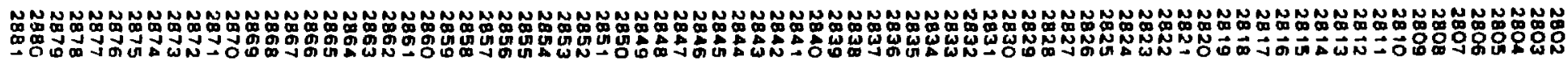
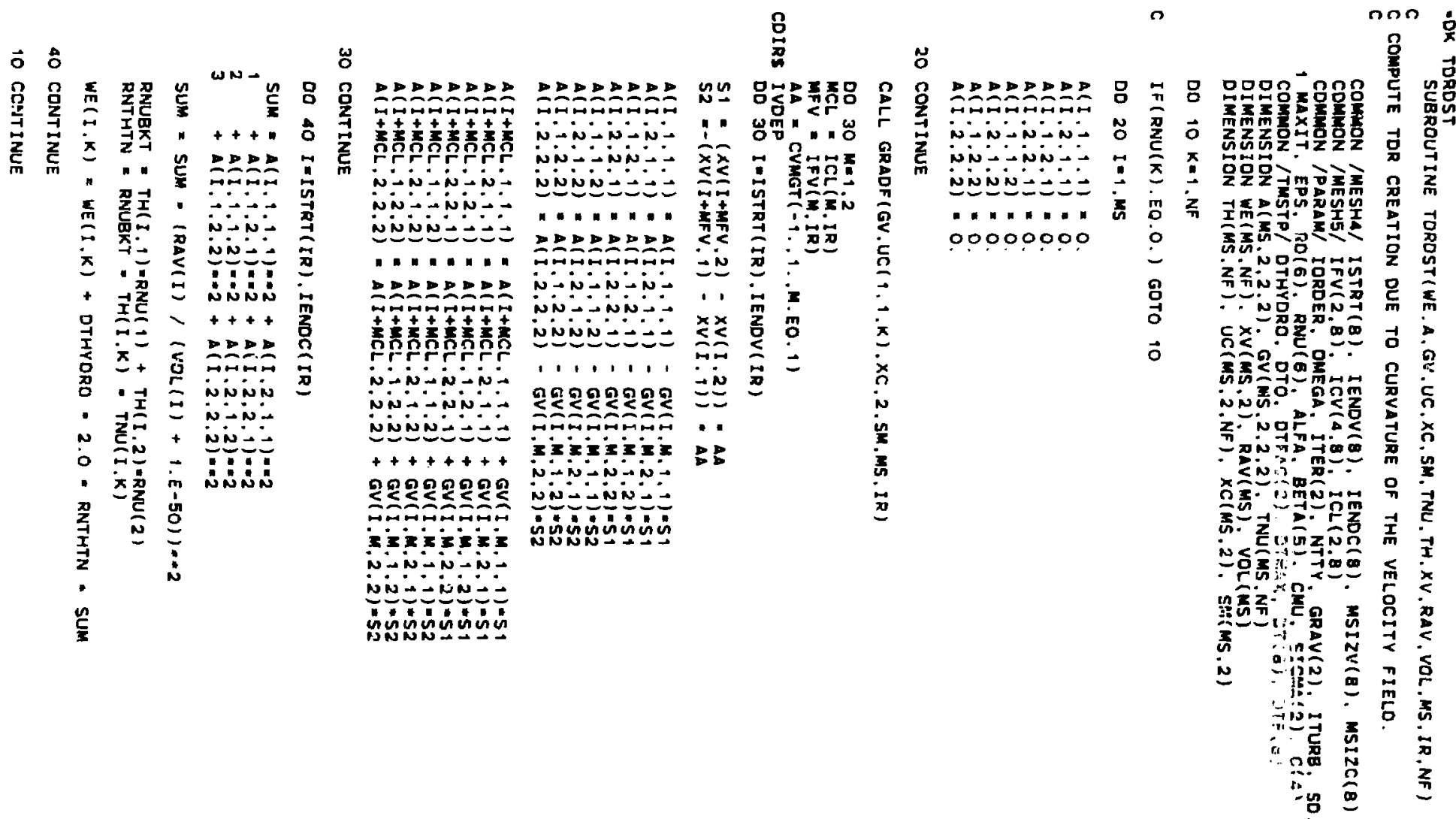


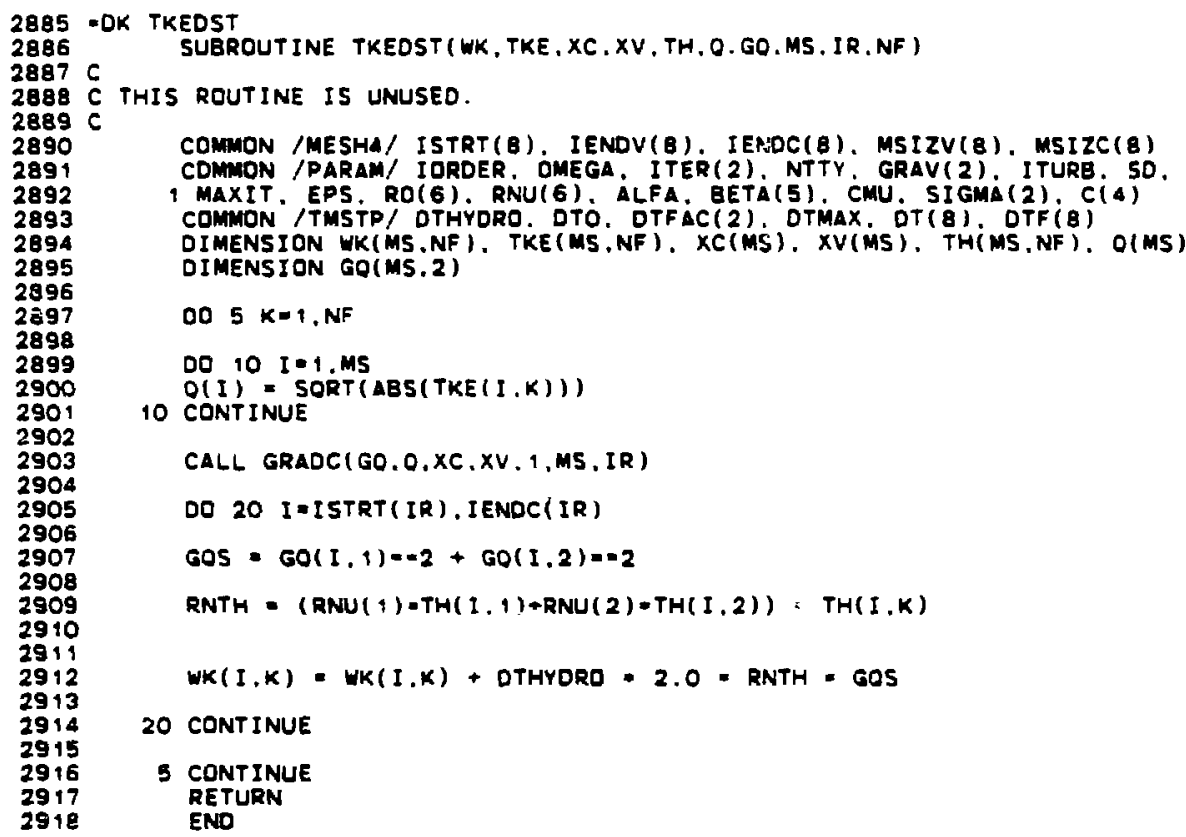




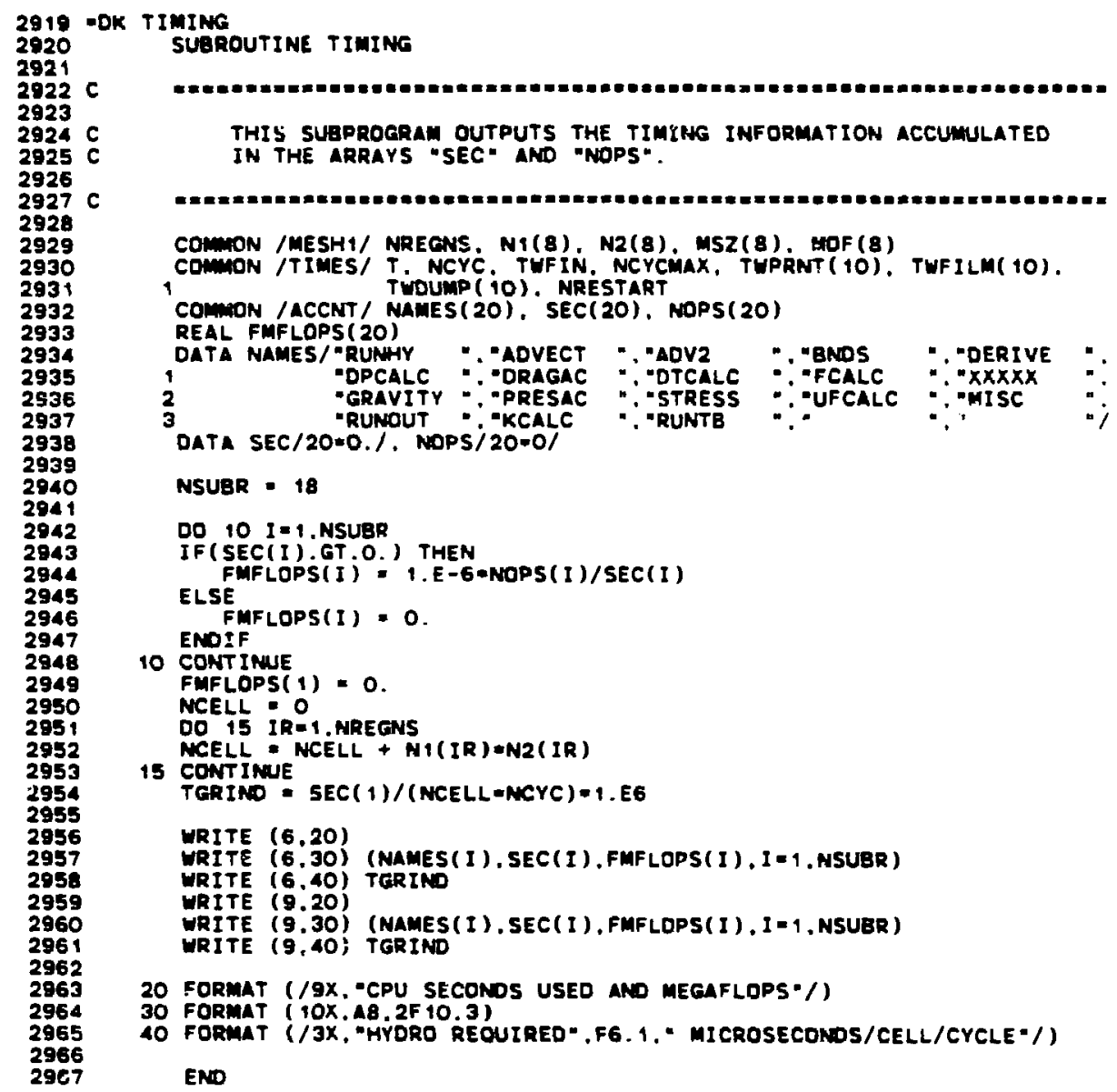




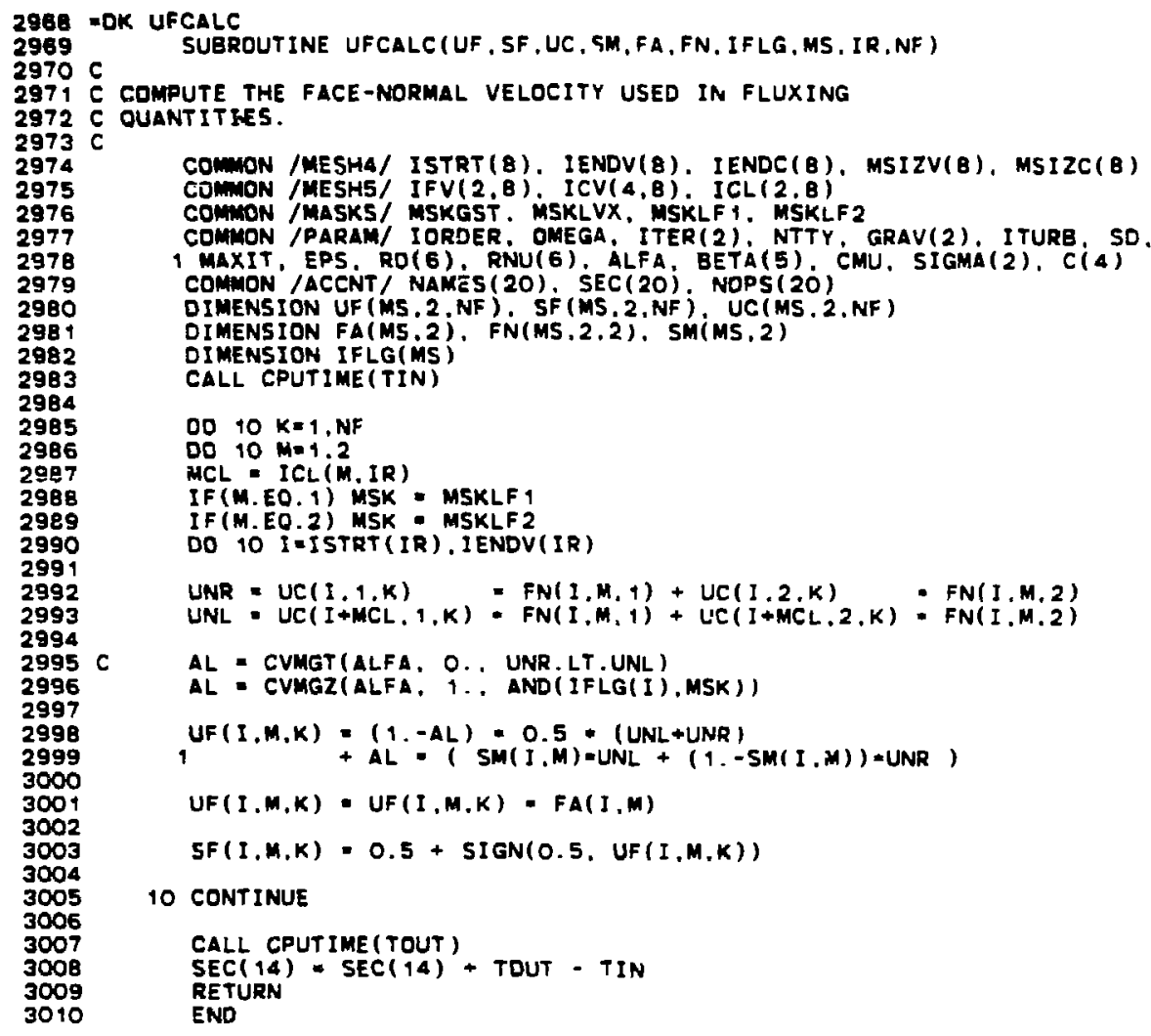




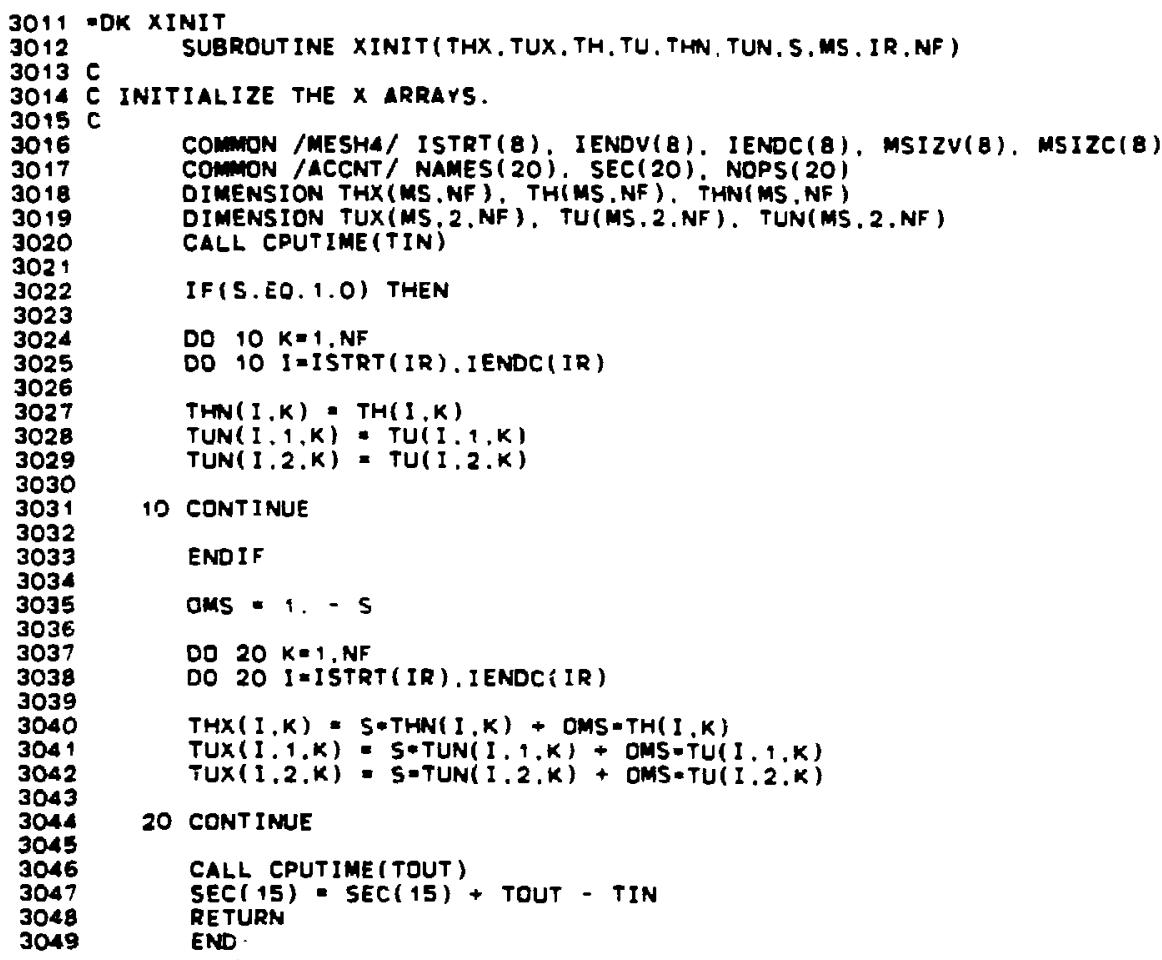

3025

3026

3027

3028

3029

3030

3031

3032

3033

3034

3035

3036

3037

3038

3039

3040

3041

3042

3043

3044

3045

3046

3047

3048

3049 


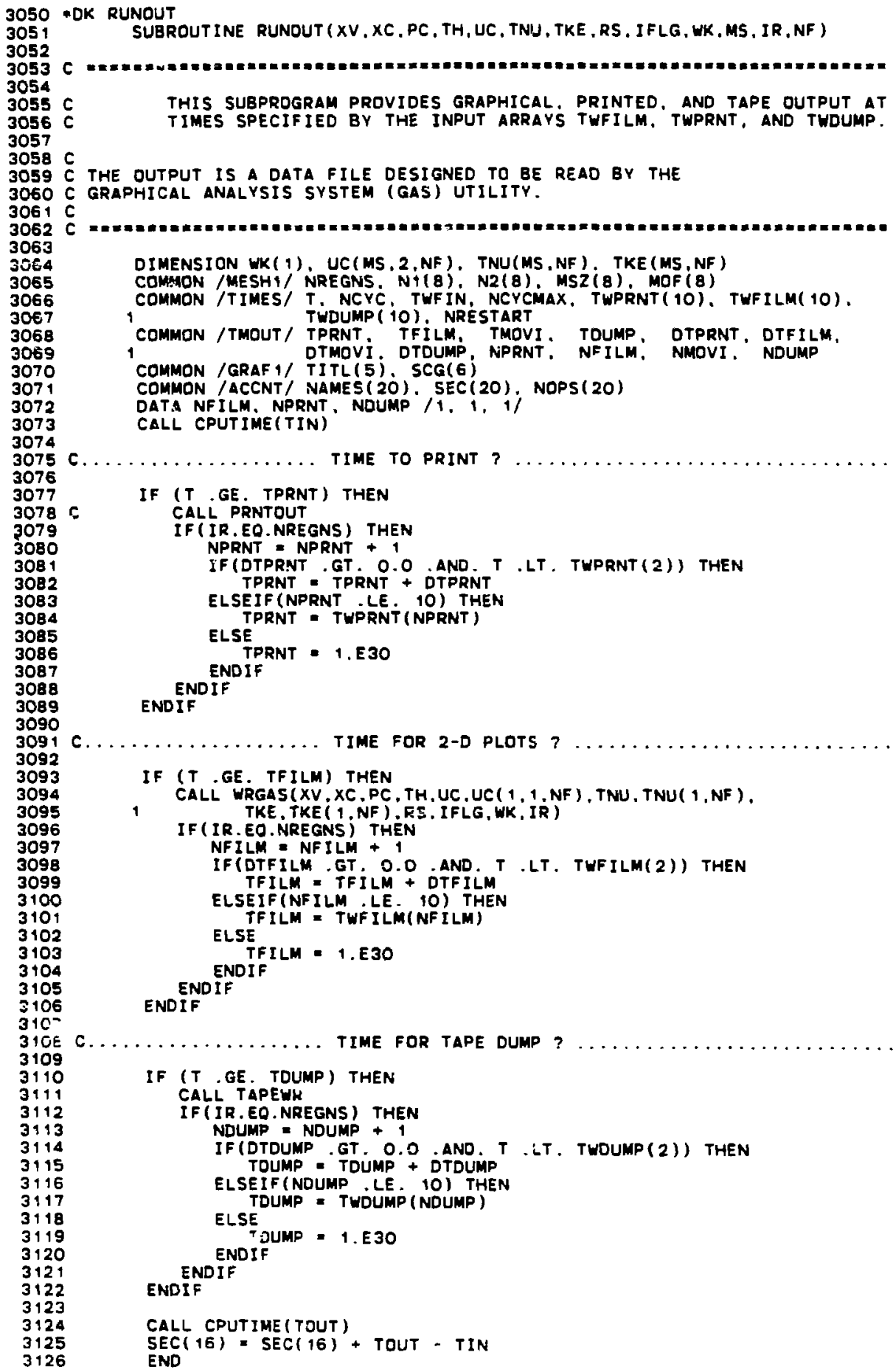




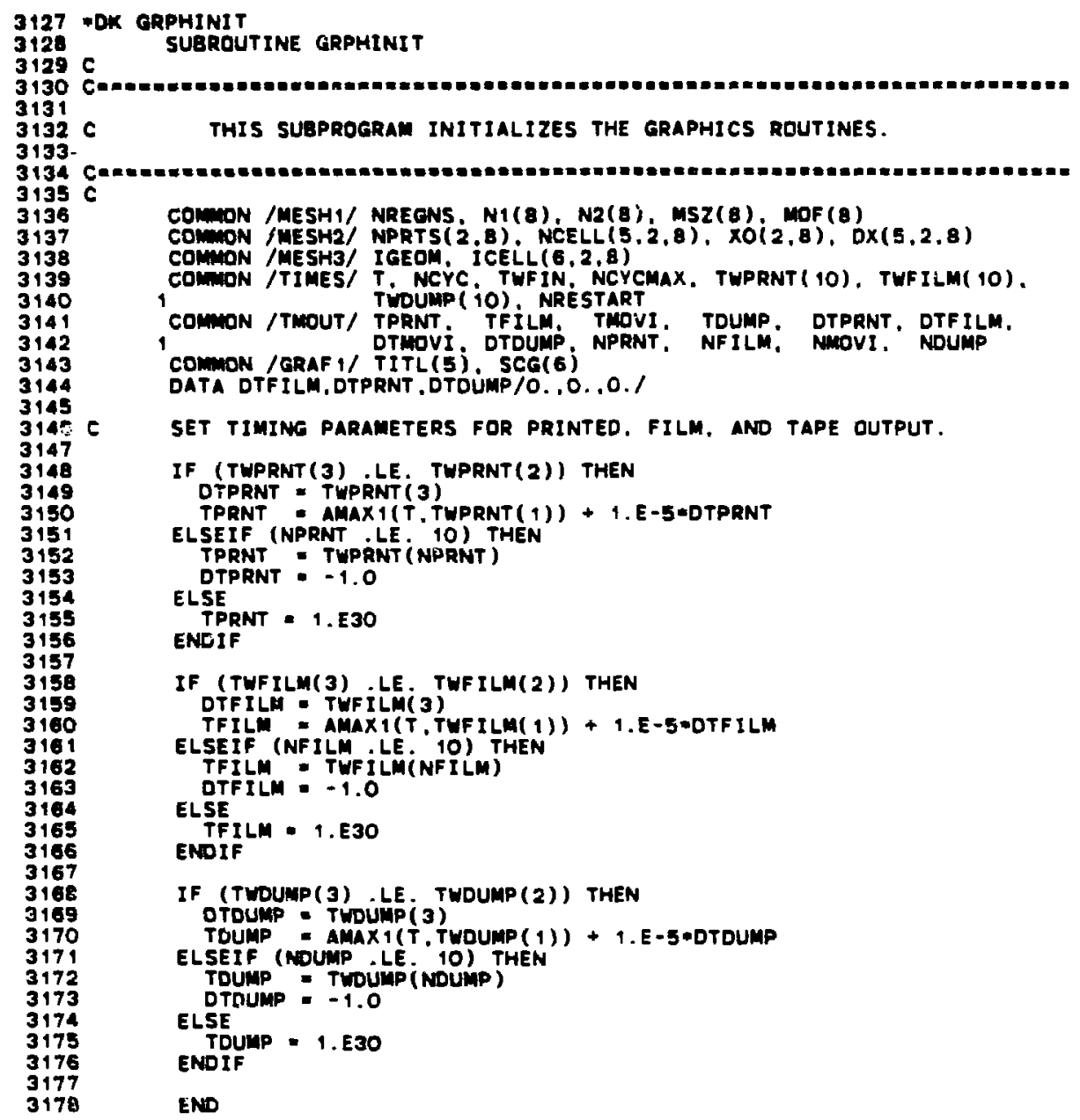




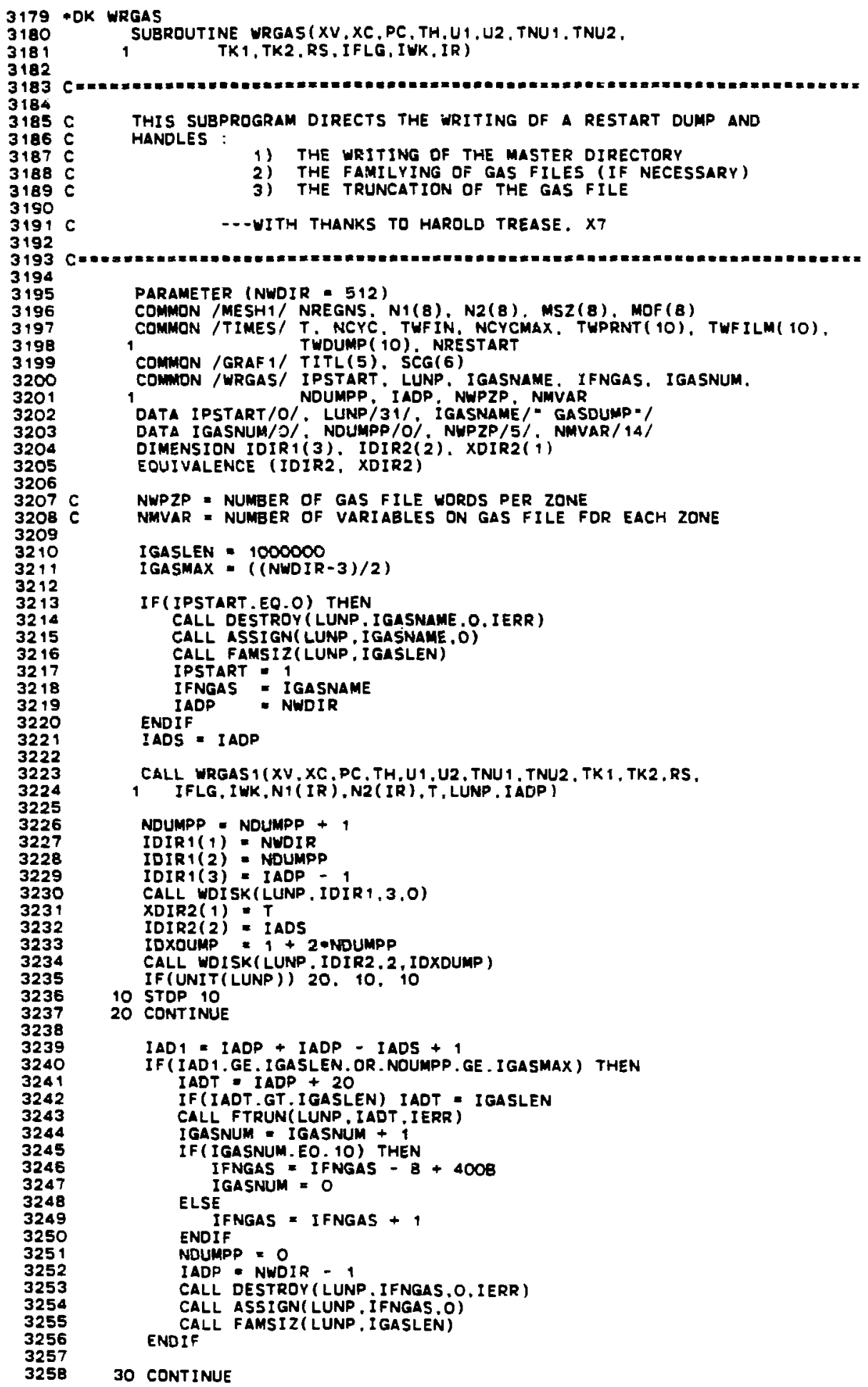


3259

3260

3261

3262

3263

3264

3265

3266

3267

3268

3269

3270

3271

3272

3273
RETURN

THIS ENTRY IS USED to TRUNCATE THE LAST GAS MDVIE DUMP.

ENTRY WRGAST

IADT = IADP + 300

IF(IADT.GT.IGASLEN) IADT IGASLEN

CALL FTRUN(LUNP, IADT , IERR)

IPSTART - O

WRITE $(6,40)$ IFNGAS. IADT

40 FORMAT("THE GAS FILE: ",AB." WAS TRUNCATED TO ".18." wORDS")

END 


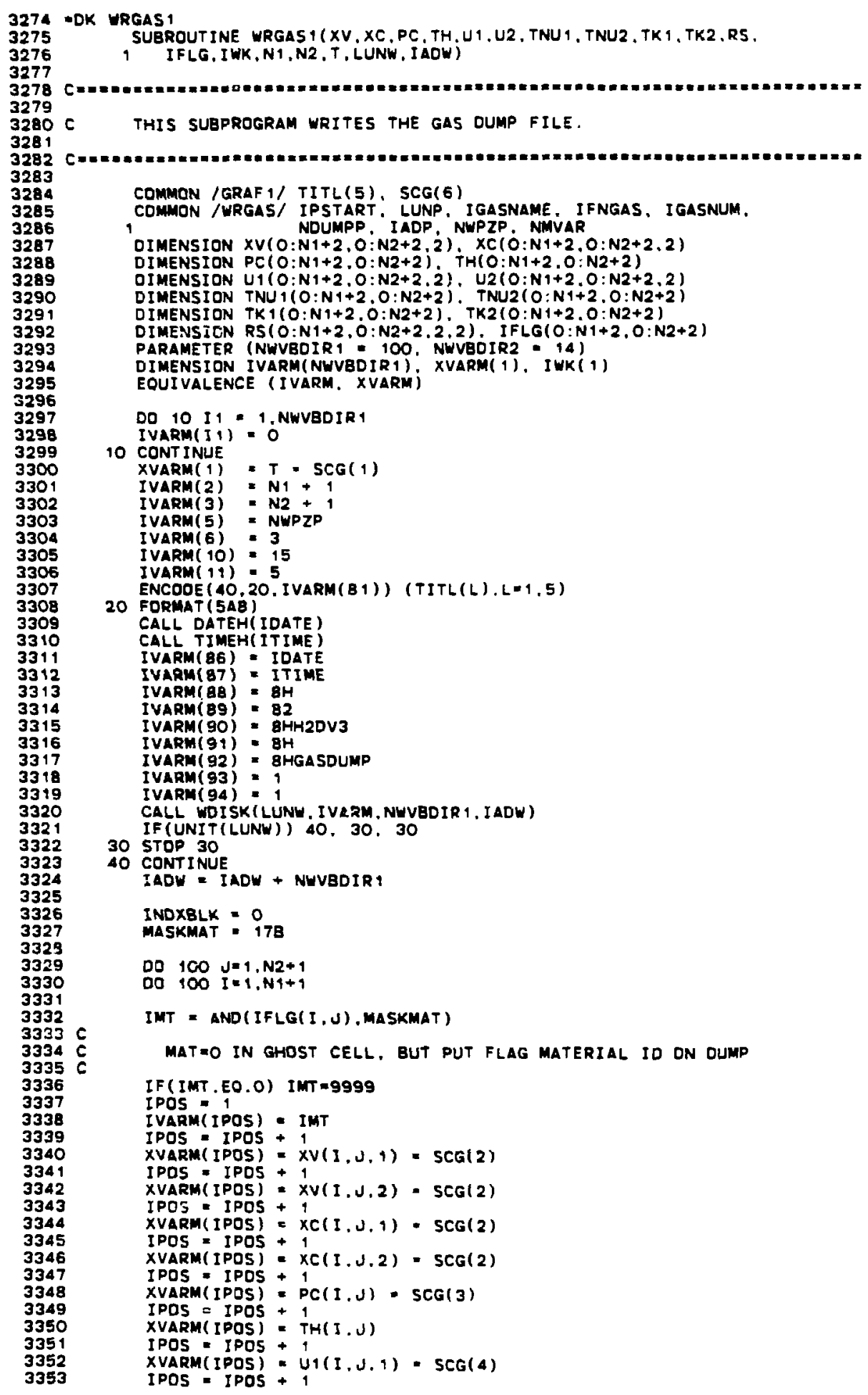




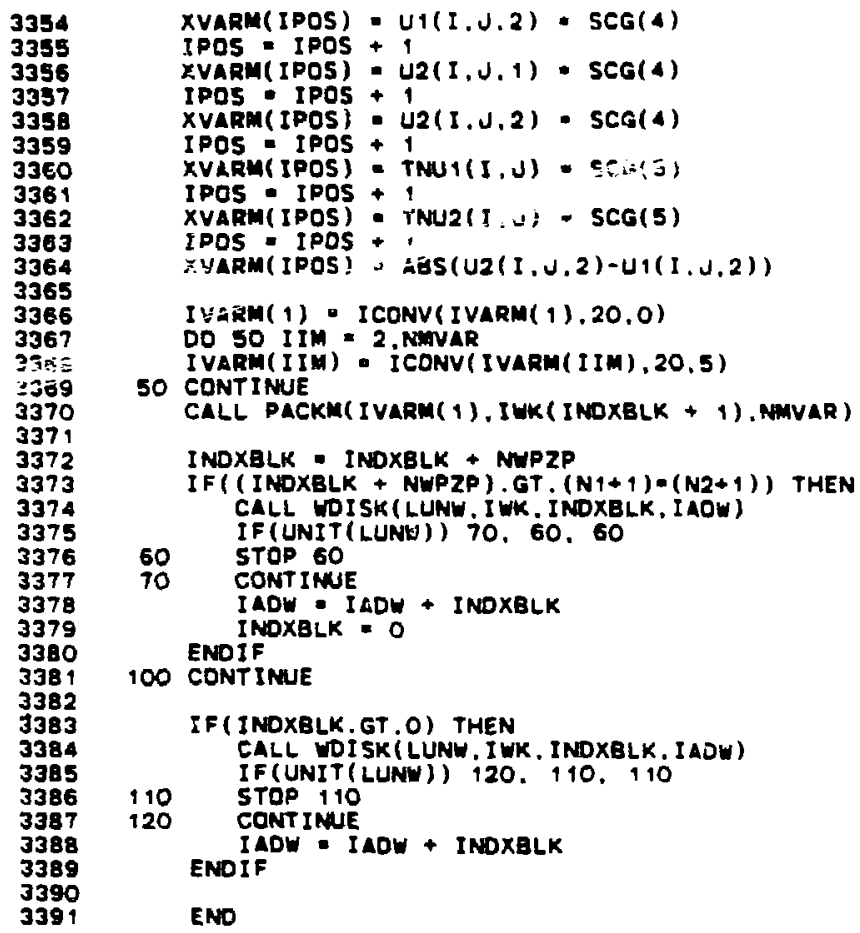




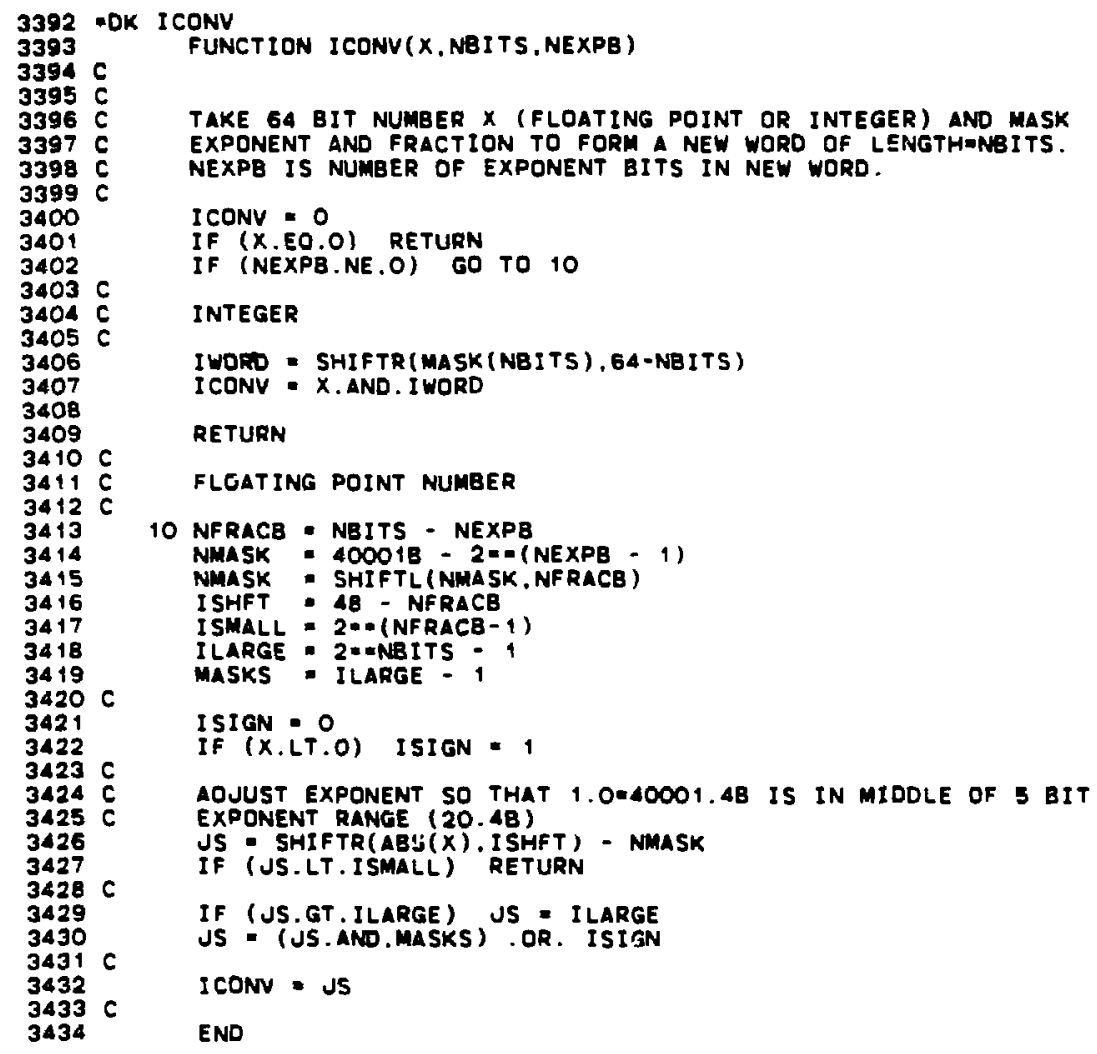




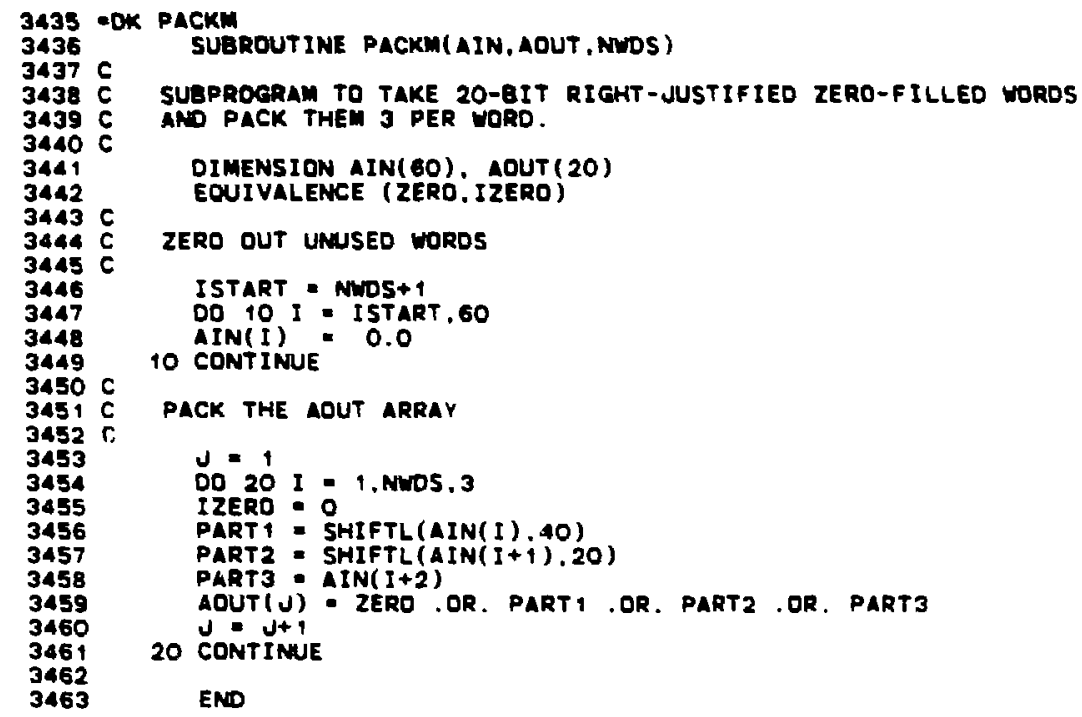




\section{Appendix $D$}

\section{Program for Solution of the Sedimentation Problem}

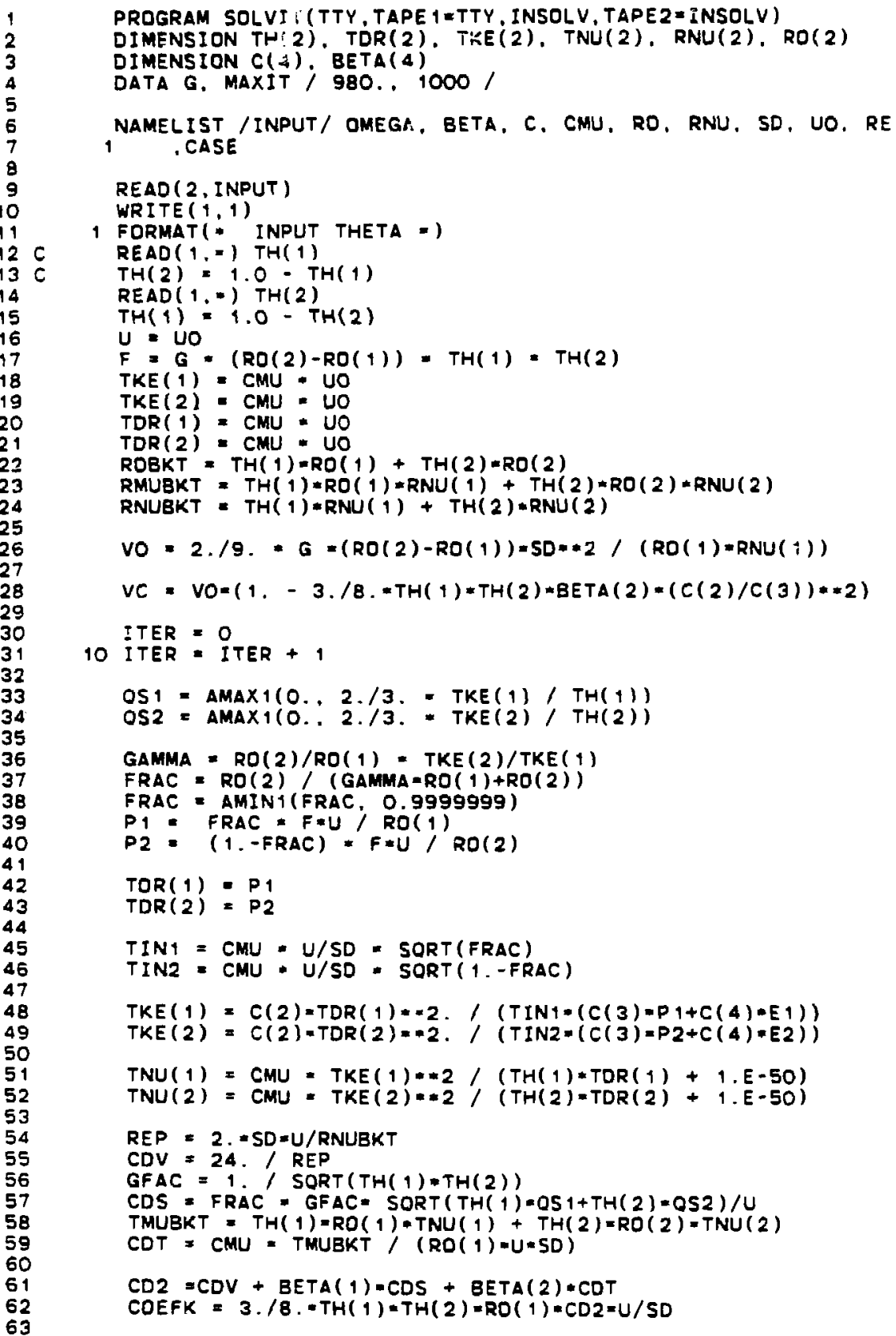




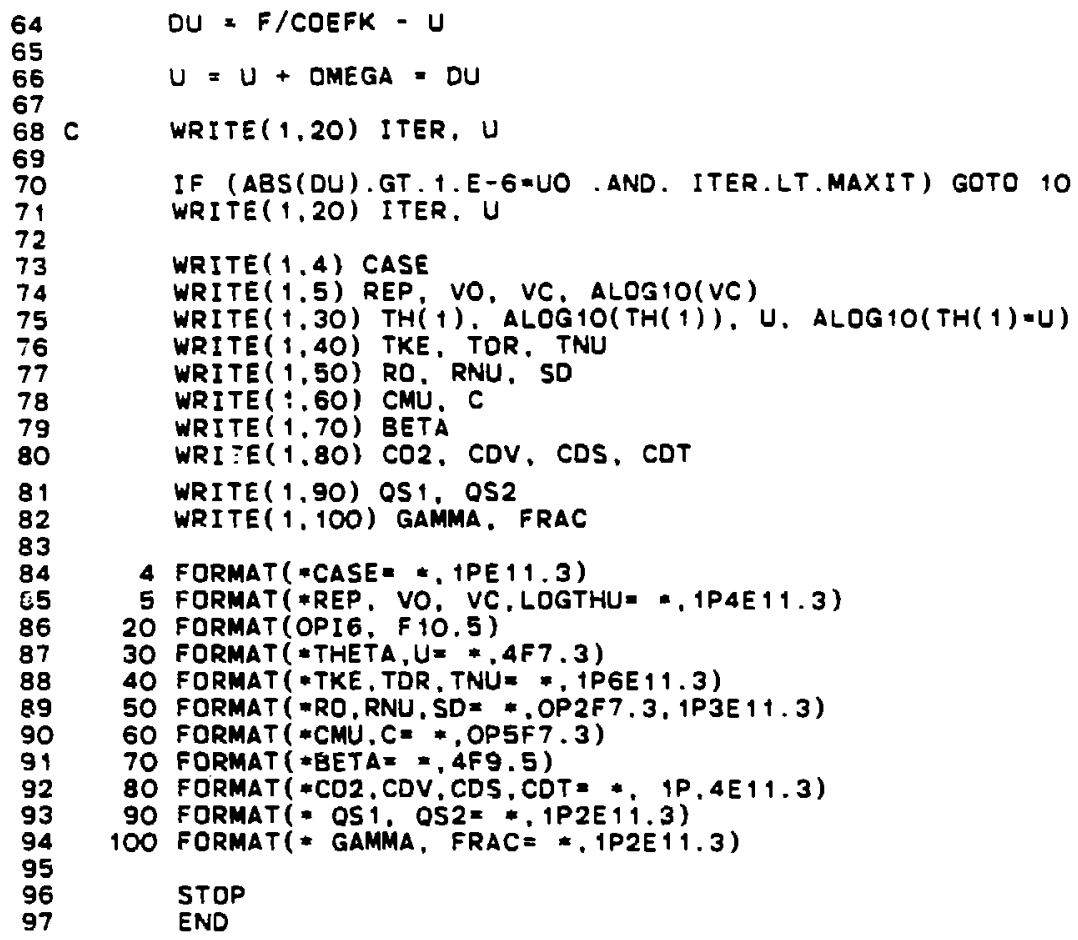

\section{SINPUT}

$S D=40 C . E-4, R O=1,225 E-3,2.7, R N U=0.145,0, U O=100$, , BETA $=0.306,1.333$

$C M U=0.0900, C=1.440,1.920,1.000$

DMEGA $=0.50 \$$

CASE $=1 . R \mathrm{RO}=2.745,2.976, R N U=6.70 E-3,0 ., S D=5.15 E-2, U O=2.48$,

$C A S E=2 \ldots R O=2.170,2,623, R N U=7,43 E-3,0 \ldots, S D=1.15 E-2, U 0=0.6525$,

CASE $=3 ., R O=2.170 .2 .923, R N U=7.43 E-3,0 \ldots, S D=4.8 O E-3, U O=3.02 E-2$,

CASE $=4 ., \quad R O=1.001,1.058$, RNU $=1.53 E-2,0, S D=9.05 E-3, U 0=6.64 E-2$.

$C A S E=5 ., R D=1,208,2.923, R N U=5,13 E-1,0 ., S D=4.8 O E-3, U O=1.39 E-2$

$C A S E=6 ., \quad R D=1.034,1.058, R N U=2.01 E-1,0 \ldots, S D=9.05 E-3, U 0=2.06 E-3$, $S D=400 . E-4, R D=1.225 E-3,2.7, R N U=0.145,0 ., U O=100 \ldots$ 